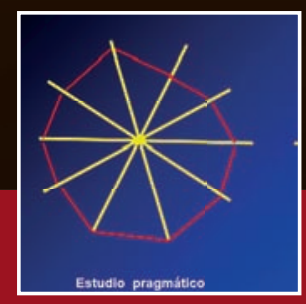

\title{
Acerca de estudios aleatorizados y observacionales
}

Jorge Thierer

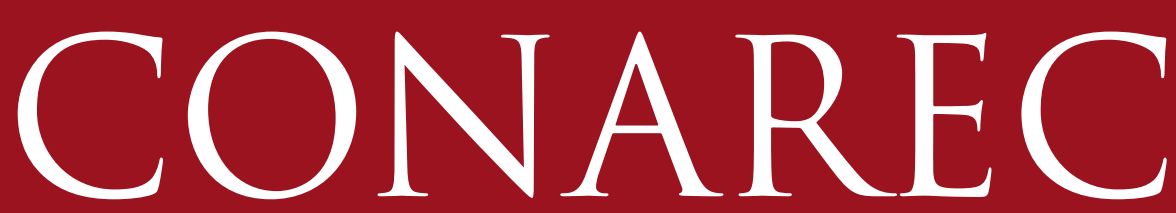

Septiembre - Octubre de 2018 - Año 33 - № 146

Complicaciones
de la ventilación
mecánica
en el posoperatorio
de cirugía cardiovascular

Fernando Ríos, Nicolás Diez

\section{MONOGRAFÍA SELECCIONADA}

Hipertensión pulmonar tromboembólica crónica en la era de la mínima invasividad

Srurs

\section{ARTÍCULOS ORIGINALES}

Estrategias intrahospitalarias de mejora del tiempo puerta-balón y evolución clínica en pacientes con infarto agudo de miocardio con supradesnivel del ST

Sigal A y cols.

Tromboembolismo pulmonar agudo en la República Argentina: protocolo del Registro CONAREC XX

Cigalini IM y cols.
Score de Goldman modificado para la evaluación de pacientes con dolor precordial en la guardia central

Vallejos Olmos Cy cols.

\section{CASOS CLÍNICOS}

Tromboembolismo pulmonar recurrente en un paciente con síndrome antifosfolipídico y trombocitopenia inducida por heparina Litwak Ny col.

Síndrome de takotsubo en paciente con cardiopatía hipertrófica Facciuto Fy cols.

\section{IMÁGENES EN CARDIOLOGÍA}

Cuando el posoperatorio se complica

Salvaggio Fy col. 


\section{Gadocor}

Nebivolol 5 - $10 \mathrm{mg}$

\section{SELECTIVO Y VASODILATADOR}

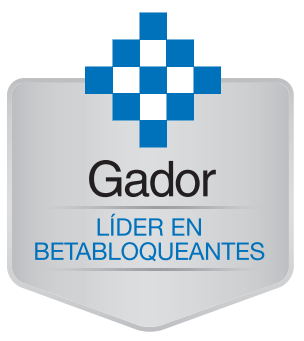

Brinda un eficaz control de la presión arterial en el paciente hipertenso²

Permite ser utilizado con seguridad en pacientes diabéticos o con síndrome metabólico

Preserva la función eréctil ${ }^{4}$

Está indicado en pacientes con EPOC

- No interfiere en el desarrollo de la actividad física del paciente ${ }^{6}$

Gadocor ${ }^{\star}$ permite que cada parte contenga la dosis correspondiente debido al diseño de sus comprimidos birranurados de $5 \mathrm{mg}$
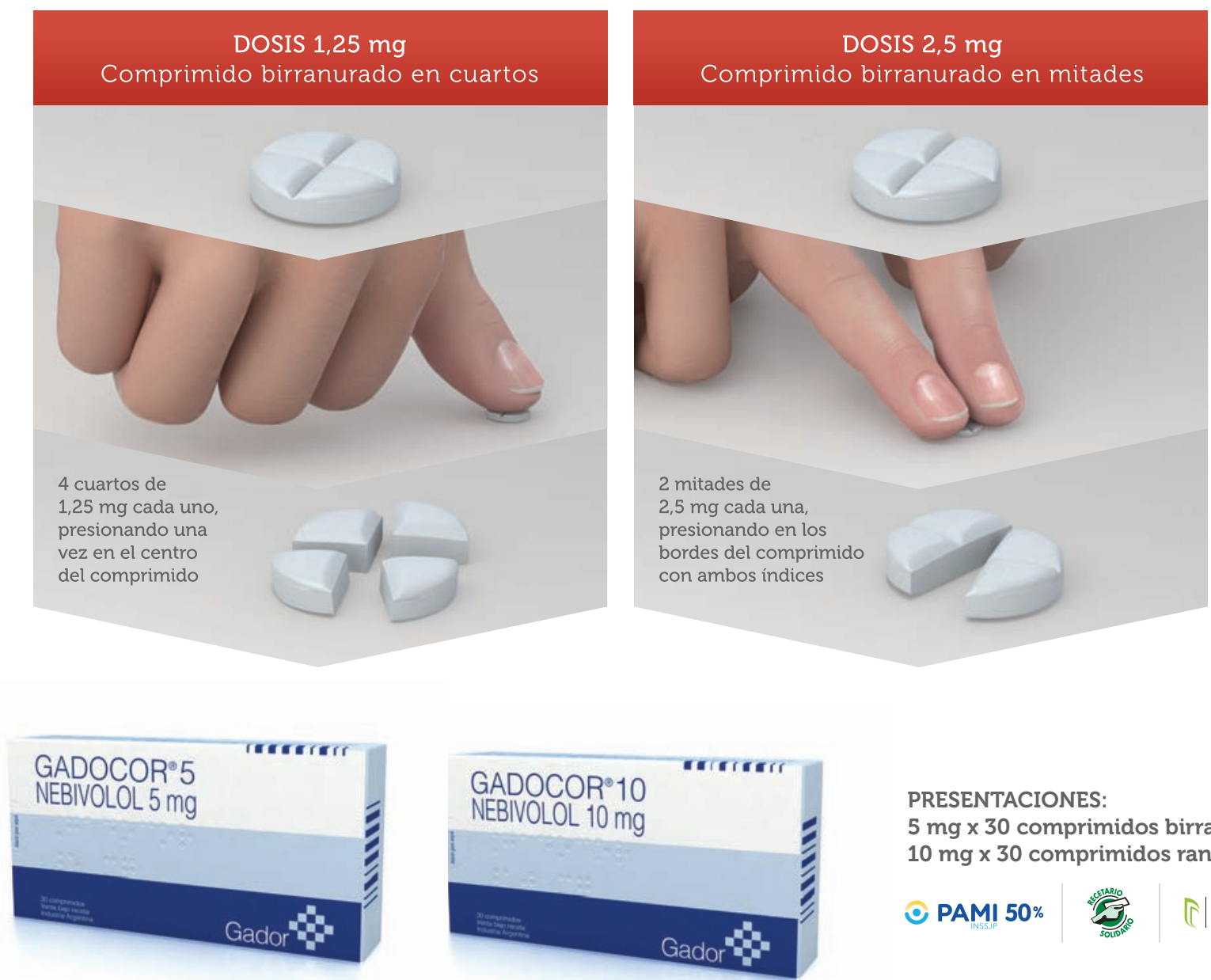

PRESENTACIONES:

$5 \mathrm{mg} \times 30$ comprimidos birranurados $10 \mathrm{mg} \times 30$ comprimidos ranurados

- PAMI 50\%

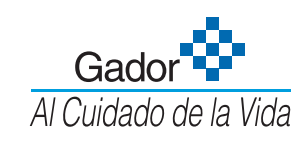




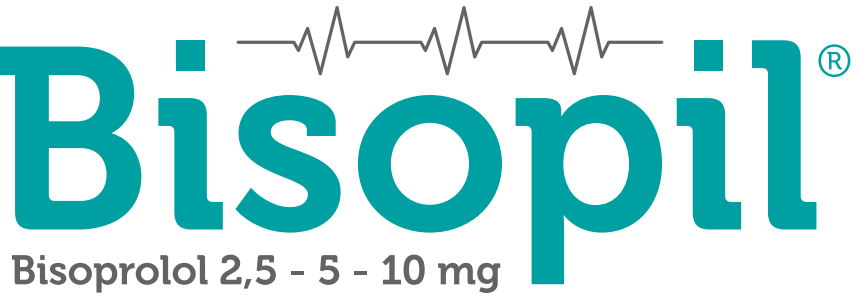

CARDIOSELECTIVO DE AMPLIA COBERTURA ${ }^{\top}$

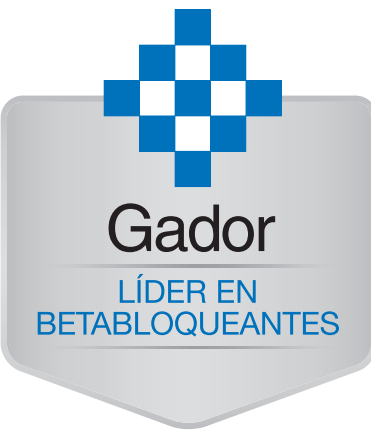

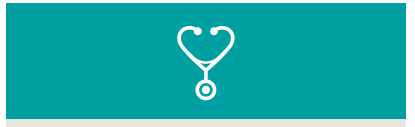

Posee alta selectividad porlos receptores $\beta 1^{2}$

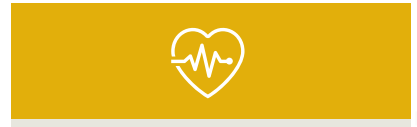

Disminuye la presión arterial de manera rápida y eficaz $^{3}$

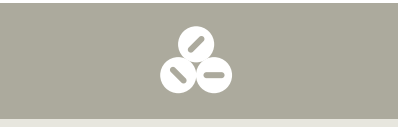

Está indicado en pacientes diabéticos compensados y/o con dislipemias $4,5,6$
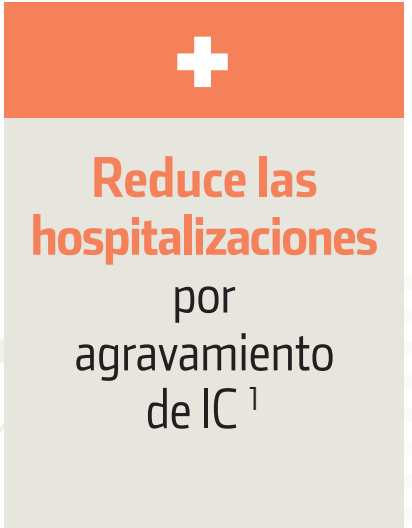

\section{TOMA IDIARIA}

BISOPIL 2,5 BISOPROLOL 2.5 mg

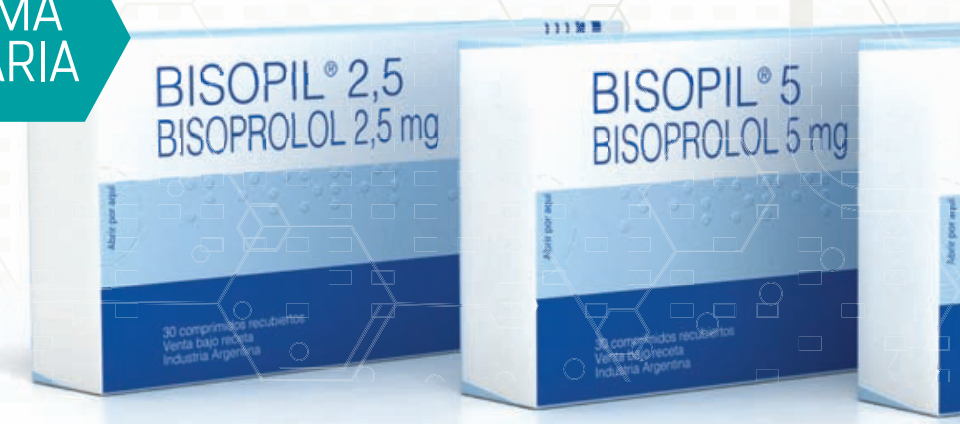

Presentaciones: 2,5 - 5 - 10 mg x 30 comprimidos
BISOPIL ${ }^{\circ} 10$

BISOPROLOL $10 \mathrm{mg}$

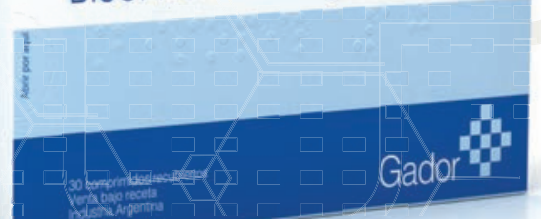

PAMI 60\%

IOMA

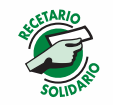




\section{Artome $\mathbf{y}^{\circ}$}

\section{Máxima prevención en la enfermedad}

cardiovascular.

$\triangleright 44 \%$ de reducción de eventos cardiovasculares en prevención primaria. ${ }^{1}$

$\checkmark$ Control lipídico en alto riesgo cardiovascular. ${ }^{2}$

$\checkmark$ Estabiliza placas coronarias ricas en lípidos. ${ }^{3}$

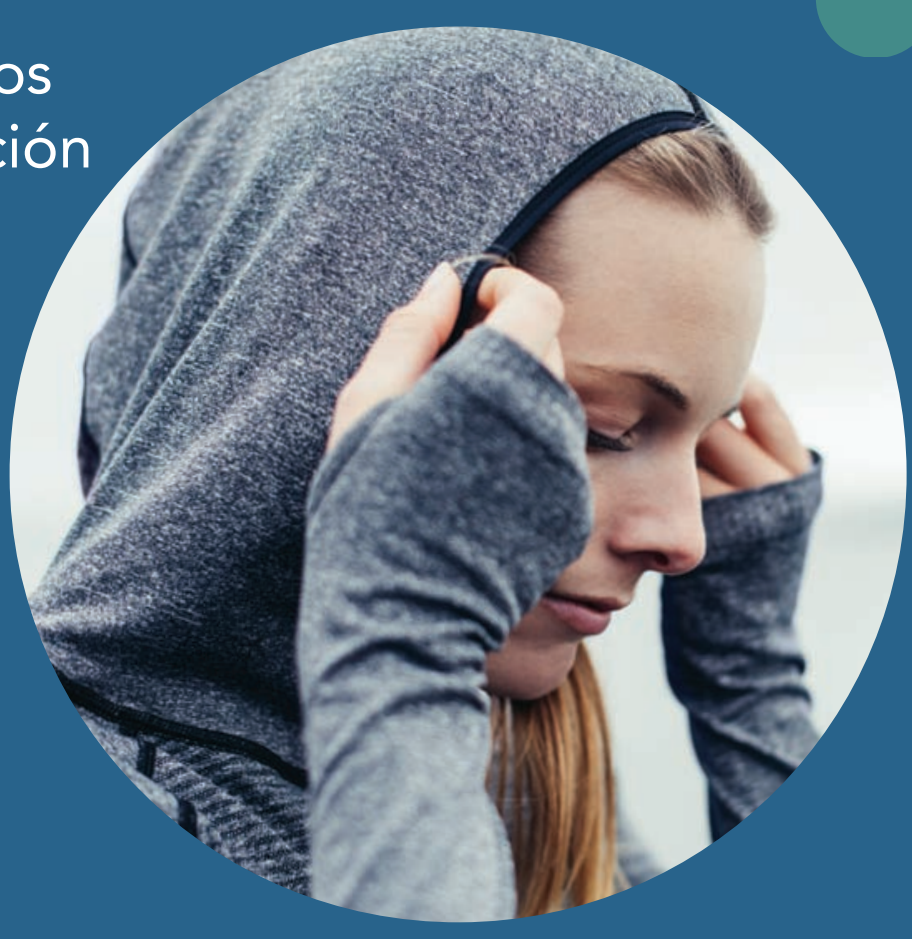

$\checkmark$ Reduce la progresión del volumen de placa de ateroma. ${ }^{4}$

$\checkmark$ Mayor eficacia para alcanzar las metas del NCEP ATP III. ${ }^{5}$

\section{REFERENCIAS BIBLIOGRÁFICAS}

1- Ridker P et al. Jupiter Trial N Eng J Med 2008; 359: 195-207 2- Shepherd J. Am J Cardiol" 2003; 91 (Suppl): 11c-19c

3- Soeda T et al. Circ J 2011; 75: 2621-7

4- Nissen S et al. Estudio Asteroid. JAMA 2006; 295: 1556-65

5- Shepherd J. Am J Cardiol: 2003; 91 (Suppl): 11c-19c
Presentaciones

Artomey ${ }^{\circledR} 5$ mg - 10 mg - 20 mg y 40 mg envases conteniendo 30 comp. recub. Artomey $^{\circledR} 10 \mathrm{mg}$ envases conteniendo 60 comp. recubiertos 


\section{DIRECTORA}

Lucrecia María Burgos

Instituto Cardiovascular de Buenos Aires | CABA

SUBDIRECTORES

Franco Facciuto

Instituto Cardiovascular de Rosario | Santa Fe

Sebastián Srur

Sanatorio Mitre |CABA

JEFE DE REDACCIÓN

Andreína Gil Ramírez

Instituto Cardiovascular de Buenos Aires|CABA

COORDINADORES

Elián Facundo Giordanino

Fundación Favaloro |CABA

Sebastián García Zamora

Hospital de Alta Complejidad "El Cruce" |

Buenos Aires

COMITÉ DE REDACCIÓN

Bruno Nicolás Acosta

Hospital Escuela de Corrientes |Corrientes

Belén Aguilar

Sanatorio Güemes | CABA

Cecilina Arnaudo

Sanatorio Mitre|CABA

Ivana Bolzán

Sanatorio Adventista del Plata | Entre Ríos

Maximiliano Echevarría

Hospital JM Cullen | Santa Fe

Fanny Fritz

Hospital Durand |CABA

Carolina Gálvez Gil

Hospital Fernández|CABA

Andrea Gil Plozzer

Hospital Naval |CABA

Florencia Giménez

Hospital JM Cullen |Santa Fe

Juan Pablo Guzmán

Hospital Británico |CABA

Martina Labaqui

Hospital Naval ICABA

Belén Leguizamón

Hospital JM Cullen |Santa Fe

Benjamín Litre

Sanatorio Mitre |CABA

Nahuel Litwak

Fundación Favaloro |CABA

Nadia Martínez

Sanatorio Güemes |CABA

Fernanda Petrucci

Hospital Fernández |CABA

Lucia Rossi

Hospital JM Cullen | Santa Fe

Federico Swedzky

Instituto Modelo de Cardiología | Córdobo

Carolina Yanzon

Sanatorio Adventista del Plata| Entre Ríos

TRADUCCIONES AL INGLÉS

María Isabel Ayala

Traductora Literaria y Técnico-Científica

COMITÉ ASESOR

Antonio Bayés de Luna

Profesor en Medicina. Investigador Senior - ICCC. Fundación de Investigación Cardiovascular. Hospital Sta. Creu y St. Pau, Barcelona, España.
Juan José Badimon, Ph.D. FACC, FAHA

Profesor en Medicina. Director, Atherothrombosis Research Unit, Cardiovascular Institute New York. Icahn School of Medicine at Mount Sinai.

Adrián Baranchuk MD FACC FRCPC FCCS

Profesor en Medicina. Queen's University. Presidente, International Society of Electrocardiology. Editor-in-Chief, Journal of Electrocardiology.

Lina Badimon

Profesora en Medicina. Director Cardiovascular Research Center (CSIC-ICCC). Hospital de la Santa Creu i Sant Pau. Vicepresidente de la European Society of Cardiology.

Julio A. Panza

Jefe de Cardiologíay Profesor de Medicina, Westchester Medical Center/New YorkMedical College.

Andrés Pérez-Riera

Médico, Universidad Nacional de Córdoba (1973). Revalidación médico, Federal University of Goiás (1979). Residencia en Medicina Interna, Hospital de Clínicas, Córdoba (1974-1975). Residencia en Cardiología, Dante Pazzanese Institute, São Paulo, Brazil (1976-1977). Título Cardiólogo "Instituto do Coração" Brazil (InCor) (1978). Título Métodos Gráficos en Cardiología, "Instituto do Coração" (InCor) (1979). Master Degree, São Paulo School of Medicine - São Paulo Hospital - Graphic Methods Area in the Cardiology Chair (1980-1981). Degree of Specialist by proficiency test in Cardiology-Brazilian Society of Cardiology (1980). Medalla de oro y Diploma, Universidad de Córdoba.

Julián Segura

Jefe de la Unidad de Hipertensión Arterial. Servicio de Nefrología Hospital Universitario 12 de Octubre. Presidente de la Sociedad Española de Hipertensión-Liga Española para la Lucha contra la Hipertensión Arterial.

David Prieto-Merino

Associate Professor, London School of Hygiene \& Tropical Medicine. Director de la Cátedra de Análisis Estadístico y Big Data de la Universidad Católica de Murcia España.

Manlio F. Márquez

Departamento de Electrofisiología, Instituto Nacional de Cardiología "Ignacio Chávez,"México D.F. Exvicepresidente de la Sociedad Latinoamericana de Estimulación Cardiacay Electrofisiología.

Fernando Peñafort

Cardiólogo universitario SAC UBA. Especialista en Electrofisiología. Universidad de la Plata SADEC. Jefe de Electrofisiología: Hospital Español de Mendoza. Clínica de Cuyo. Hospital Santa Isabel de Hungría. Médico de planta, Servicio de Cardiología. Hospital Lagomaggiore, Mendoza. Miembro titular de la Federación Argentina de Cardiología y Sociedad Argentina de Cardiología.

Daniel Piskorz

Presidente Federación Argentina de Cardiología 2017. Presidente Sociedad Argentina de Hipertensión Arterial 2011-2013. Director Centro de Investigaciones Cardiovasculares del Sanatorio BritánicoSA de Rosario.

Eduardo R. Perna

Jefe División de Insuficiencia Cardíaca e Hipertensión Pulmonar Unidad de Cuidados Intensivos Coronarios Instituto de Cardiología "J. F. Cabral", Corrientes. Argentina.

Mario Bendersky

Profesor titular Farmacología Aplicada UNC

Director maestría HTA, Univ Católica Córdoba. Jefe unidad HTA Inst. Modelo Cardiología, Cba.

Néstor A. Vita

Jefe de Cardiologia del Hospital Italiano de Rosario. Ex presidente de Federación Argentina de Cardiología

Jorge Thierer

Jefe Unidad Insuficiencia Cardíaca CEMIC. Director Asociado

Revista Argentina de Cardiología

\section{Ricardo Iglesias}

Ex Presidente CONAREC Expresidente Sociedad Argentina de Cardiología. Expresidente Fundación Cardiológica Argentina. Fellow American College of Cardiology.

Hernán C. Doval

Médico de Cardiología del Htal. Italiano de Bs. As. Director de la Revista Argentina de Cardiología

Carlos Taje

Jefe de Cardiología Hospital El Cruce. Expresidente Sociedad Ar gentina de Cardiología.

Hugo Grancelli

Jefe del Servicio de Cardiología del Sanatorio Finochietto Director Carrera de Especialista en Cardiología. Universidad de Buenos Aires. Expresidente de la Sociedad Argentina de Cardiología.

José Horacio Casabé FACC, PhD

Jefe de Cardiología ICYCC. Hospital Universitario Fundación Favaloro. Miembro titular Sociedad Argentina de Cardiología. Laura Schreier

Doctora en Bioquímica, Profesora Titular Facultad de Farmacia y Bioquímica-UBA Jefe del Laboratorio de Lípidos y Aterosclerosis Directora del Departamento de Bioquímica Clínica, Hospital de Clinicas, UBA. Secretaria Asuntos Internacionales de Sociedad Argentina de Lípidos, Miembro Comité Asesor del Consejo de Aterosclerosis y Trombosis, Sociedad Argentina de Cardiología.

Martín Donato

Profesor en Medicina. Instituto de Fisiopatología Cardiovascular. Facultad de Medicina, Universidad de Buenos Aires. Investigador CONICET

Alejandro Macchia

Fundación GESICA.

Roberto Héctor lermoli

Profesor en Medicina. Director de Docencia e Investigación, Hospital de Clínicas, UBA. Director del Dpto. de Medicina, Facultad de Medicina, UBA

Raul Borracci

Profesor de Bioestadística, Facultad de Ciencias Biomédicas, Universidad Austral. Servicio de Cirugía Cardiovascular, Facultad de Medicina, UBA. Director Adjunto de la Revista Argentina de Cardiología. Coordinador del Comité de Bioética de la Sociedad Argentina de Cardiología.

Hernán Cohen Araz

Cardiólogo CEMIC y centro médico Pilares. Magister en Efectividad Clínica y Sanitaria. Miembro titular, integrante del área de investigación y exdirector del Consejo de Emergencias Cardiovasculares. Sociedad Argentina de Cardiología. Exjefe de Cardiología FLENI.

COORDINADOR REVISIÓN ANUAL

Guillermo R. Chiappero

Hospital Fernández|CABA

MIEMBROS HONORARIOS

Dr. René Favaloro +

Dr. Carlos Bertolasi +

Dr. Arnaldo Angelino

SECRETARIA CONAREC

Mariela Tolcachier

Tel: (011) 1536772989

secretariaconarec@yahoo.com.ar

CORRESPONDENCIA

www.revistaconarec.com.a

conarecrevista@gmail.com

SEDE SOCIAL

Azcuénaga 980 - CABA 


\section{AUTORIDADES - CONAREC 2018}

\section{PRESIDENTE}

Sebastián García Zamora

Hospital de Alta Complejidad en Red "E

Cruce"

VICEPRESIDENTE

Hernán Pulenta

Hospital Lagomaggiore | Mendoza

TESORERA

Eliane Sasovsky

Trinidad Mitre |CABA

PROTESORERO

Pablo Schmidt

Sanatorio Güemes |Chaco

SECRETARIO

Emmanuel Scatularo

Fundación Favaloro $\mid C A B A$

PROSECRETARIO

Mariano Visconti

Instituto de Cardiología de Corrientes

VOCALES

\section{CABA y Gran Buenos Aires}

Macarena Cousirat Liendro

Clínica Bazterrica |CABA

Matías Anuati

UAI $\mid C A B A$

María Esperanza Raimundo

Malvinas Argentinas | Gran Buenos Aires

Chaco

Matías Villacorta

Sanatorio Güemes
Víctor Ramírez

Clínica del Valle

Córdoba

Cintia Sánchez

Sanatorio Allende

\section{Corrientes}

Mariana Andrea Candia

Instituto de Cardiología de Corrientes

Entre Ríos

Iván Vilar Evequoz

Hospital San Martin de Paraná

Formosa

Norali Miranda

Hospital de Alta Complejidad "Juan Domingo Perón"

Jujuy

Carolina Galarza

Sanatorio Nuestra Señora del Rosario

La Rioja

Juan Santillán

NCOR

\section{Mendoza}

Romano Trionfi

Hospital Español

Misiones

Angelo Zubrzycki

Hospital SAMIC El Dorado

\section{Neuquén}

Soledad Orazi

Hospital Provincial Neuquén

\section{Rosario}

María del Carmen Salvia

Hospital Provincial

Salta

Valentina Botelli

Hospital San Bernardo

\section{San Juan}

Carolina Robles

ICVO (Instituto Cardiovascular del Oeste)

\section{Santa Fe}

Leonel Moyano

Sanatorio Cullen

DIRECTORA REVISTA

Lucrecia Burgos

Instituto Cardiovascular de Buenos Aires |

CABA

SUBDIRECTORES

Franco Facciuto

Instituto Cardiovascular de Rosario I Santa Fe

Sebastián Srur

Sanatorio Mitre $\mid$ CABA

PÁGINA WEB

Director: Lin YuShan

Hospital Durand |CABA

Subdirector (CABA): Juan Pablo Cattáneo Sagrado Corazon | CABA

\section{COMITÉ CIENTÍFICO}

Ana Lía Gamarra

Hospital Italiano de Buenos Aires |CABA

Paula Berlien

Hospital "I. M. Cullen" | Santa Fe

Alan Sigal

Instituto Cardiovascular de Buenos Aires

CABA

ÓRGANO DE FISCALIZACIÓN

Y PERMANENCIA

Ignacio Cigalini

Instituto Cardiovascular de Buenos Aires | CABA

Darío lgolnikof

Hospital Austral| Buenos Aires

STAFF

Mariela Tolcachier

Secretaria Presidencia

\section{SEDES DE JORNADAS}

$\begin{array}{ll}\text { 1980: I } & \text { Buenos Aires } \\ \text { 1981: II } & \text { Buenos Aires } \\ \text { 1982: III } & \text { Buenos Aires } \\ \text { 1983: IV } & \text { Buenos Aires } \\ \text { 1984:V } & \text { Buenos Aires } \\ \text { 1985: VI } & \text { Buenos Aires } \\ \text { 1986: VII } & \text { Rosario } \\ \text { 1988: IX } & \text { San Juan } \\ \text { 1990: X } & \text { Buenos Aires } \\ \text { 1991: XI } & \text { Buenos Aires } \\ \text { 1992: XII } & \text { Córdoba } \\ \text { 1994: XIV } & \text { Rosario }\end{array}$

$\begin{array}{ll}\text { 1995: XV } & \text { Mendoza y San Juan } \\ \text { 1996: XVI } & \text { Tucumán } \\ \text { 1997: XVII } & \text { Corrientes } \\ \text { 1998: XVIII } & \text { San Juan } \\ \text { 1999: XIX } & \text { Buenos Aires } \\ \text { 2000: XX } & \text { Córdoba } \\ \text { 2001: XXI } & \text { Rosario } \\ \text { 2002: XXII } & \text { Entre Ríos } \\ \text { 2003: XXIII } & \text { Tucumán } \\ \text { 2004: XXIV } & \text { Corrientes } \\ \text { 2005: XXV } & \text { Córdoba } \\ \text { 2006: XXVI } & \text { San Juan }\end{array}$

$\begin{array}{ll}\text { 2007: XXVII } & \text { Buenos Aires } \\ \text { 2008: XXVIII } & \text { La Rioja } \\ \text { 2009: XXIX } & \text { Mendoza } \\ \text { 2010: XXX } & \text { Jujuy } \\ \text { 2011: XXXI } & \text { Córdoba } \\ \text { 2012: XXXII } & \text { Santa Fe } \\ \text { 2013: XXXIII } & \text { Rosario } \\ \text { 2014: XXXIV } & \text { Mar del Plata } \\ \text { 2015: XXXV } & \text { Mendoza } \\ \text { 2016: XXXVI } & \text { Rosario } \\ \text { 2017: XXXVII } & \text { Santa Fe }\end{array}$

\section{e conAREC}

\section{Fundada en 1985 por el Dr. Arnaldo Adrián Angelino}

\section{Publicación bimestral. @ CONAREC}

Registro de la Propiedad Intelectual № 572790. Reservados todos los derechos. Ninguna parte de esta publicación puede ser reproducida en forma o medio alguno, electrónico o mecánico, incluyendo fotocopias, grabaciones y otros sistemas de información sin la autorización por escrito del titular del Copyright.

ISSN: 0329-0433 | ISSN: 1853-2357 (en línea). Propietario: Consejo Nacional de Residentes de Cardiología (CONAREC). Se distribuye exclusivamente entre los profesionales de la medicina. Es una publicación del CONAREC, Azcuénaga 980 C1122AAJ |Ciudad Autónoma de Buenos Aires.

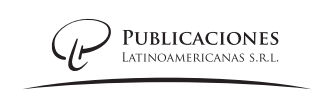

PRODUCCIÓN EDITORIAL, COMERCIAL Y GRÁFICA

PUBLICACIONES LATINOAMERICANAS S.R.L.

Piedras $13332^{\circ} \mathrm{C}$ C1240ABC Ciudad Autónoma de Buenos Aires | Argentina tel/fax (5411) 5217-0292 | e-mail info@publat.com.ar | http://www.publat.com.ar 


\section{PAXOND}

LOSARTÁN I HIDROCLOROTIAZIDA

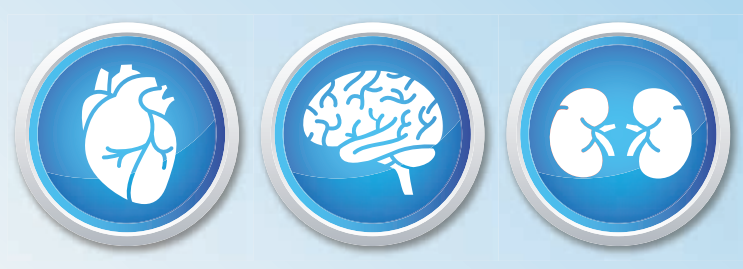

\section{Eficacia antihipertensiva con protección de órgano blanco ${ }^{1-4}$}

\section{PAMI 80\% IOMA}

Paxon` 50 - 100: envases por 30 y 60 comprimidos recubiertos ranurados conteniendo losartán.

Paxon XRं: envase por 30 comprimidos de liberación controlada conteniendo losartán.

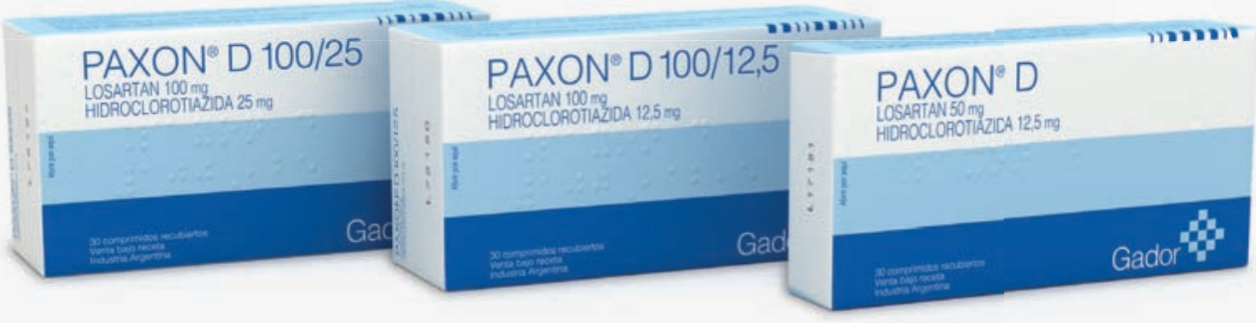

PAMI 60\%

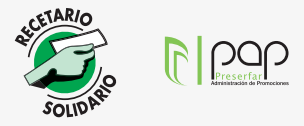

Paxon $\mathrm{D}^{\mathrm{Q}}$ : envases por 30 y 60 comprimidos recubiertos ranurados conteniendo losartán e hidroclorotiazida.

Paxon $D^{\oplus} 100 / 12,5-100 / 25$ : envases por 30 comprimidos recubiertos ranurados conteniendo losartán e hidroclorotiazida.

Referencias: 1. Little WC y col; Effect of losartan and hydrochlorothiazide on exercise tolerance in exertional hypertension and left ventricular diastolic dysfunction; Am J Cardiol 2006 Aug 1;98(3):383-5. 2. Moen MD, Wagstaff AJ; Losartan: a review of its use in stroke risk reduction in patients with hypertension and left ventricular hypertrophy; Drugs 2005;65(18):2657-74. 3. Brenner BM y col; RENAAL Study Investigators. Effects of losartan on renal and cardiovascular outcomes in patients with type 2 diabetes and nephropathy; N Engl J Med 2001 Sep 20;345(12):861-9. 4. Elliott WJ, Meyer PM; Incident diabetes in clinical trials of antihypertensive drugs: a network meta-analysis; Lancet 2007 Jan 20;369(9557):201-7.

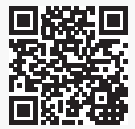

Para más información sobre PAXON ${ }^{\circledR}, \mathrm{PAXON}^{\otimes} \mathrm{XR}$ y $\mathrm{PAXON}{ }^{\circledR} \mathrm{D}$ visite www.gador.com.ar
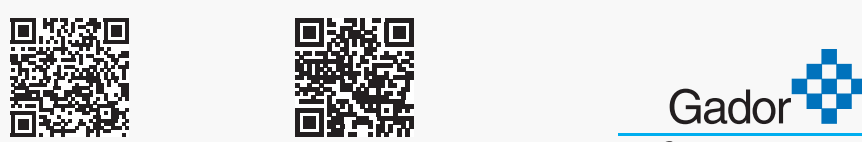

$\overline{\text { Al Cuidado de la Vida }}$ 
CIUDAD AUTÓNOMA DE BUENOS AIRES Hospital General de Agudos Ramos Mejía Complejo Médico Policial "Churruca-Visca" Hospital Británico de Buenos Aires

Sanatorio "Sagrado Corazón"

Hospital Universitario Fundación Favaloro

Hospital Español (Univ. Abierta Interamericana)

Hospital General de Agudos Durand

Hospital General de Agudos Rivadavia

Hospital Alemán

Hospital Naval Buenos Aires P. Mallo

Sanatorio de la Trinidad Mitre

Sanatorio Municipal "Dr. Julio Méndez"

Sanatorio Otamendi y Miroli

Sanatorio Colegiales

Hospital General de Agudos Santojanni

Hospital de Clínicas "José de San Martín"

Sanatorio Güemes

Hospital Militar Central

Instituto Denton Cooley

Sanatorio Centro Gallego

Hospital General de Agudos Argerich

Policlínico Bancario

Hospital César Milstein

Clinica Bazterrica

Clinica Santa Isabel

Hospital Italiano de Buenos Aires

Instituto Cardiovasc. de Buenos Aires "ICBA"

Hospital General De Agudos Fernández

Hospital Aeronáutico Central

GRAN BUENOS AIRES

Hospital Central de San Isidro

Sanatorio Modelo Quilmes

Hptal. Gral. de Agudos "Eva Perón" (Ex Castex)

Hospital Interzonal de Agudos Gandulfo

Hospital "Juan D. Perón" - Malvinas Argentinas

Hospital Universitario Austral

Clínica IMA - Adrogué

Hospital Municipal "Dr. Larcade"- San Miguel

Sanatorio San Miguel

Hospital Nacional Posadas

Hospital Interzonal Gral de Agudos Evita - Lanús Clínica del Valle

Hospital El Cruce - Florencio Varela

CATAMARCA

$\mathrm{CHACO}$

de Urgencias

CÓRDOBA

Clínica Chutro

Clínica Sucre
PROVINCIA DE BUENOS AIRES

Htal Gral de Agudos "J. de San Martín"- La Plata Htal de Ag.y Crónicos "S. J. de Dios" - La Plata Htal Gral de Agudos "R. Rossi" - La Plata

Htal Gral de Agudos "O. Alende"- Mar del Plata Htal Privado de la Comunidad - Mar Del Plata

Htal Gral de Agudos"J. Penna"- Bahía Blanca

Clinica Privada Fumeba - Hospital Privado del

Sur - Bahía Blanca

Hospital San Nicolás de los Arroyos

Htal Gral Agudos Dr. Paroissien - I. Casanova

Instituto de Cardiología Intervencionista

Instituto del Corazón "CORDIS"

Gran Hospital Dr. Julio C Perrando

Sanatorio Güemes

Centro Cardiovascular del Nordeste - Clínica

Clínica Privada Vélez Sarfield

Clínica Universitaria Reina Fabiola

Hospital Italiano de Córdoba

Instituto Modelo de Cardiología

Sanatorio Allende

Sanatorio Mayo

Hospital Córdoba

Instituto Modelo de Cardiología Privado

Hospital Aeronáutico Córdoba

Hospital Privado Centro Médico de Córdoba

Hospital "San Roque"

Clínica Romagosa

Sanatorio del Salvador

Sanatorio Parque

Clinica Fusavim Privada

Clínica Privada de Especialidades - Villa María Instituto Médico Río Cuarto

Hor

CORRIENTES Sanatorio Británico

Sanatorio Delta

Hospital de Emergencias "Dr. C. Álvarez"

Hospital Escuela "José Francisco de San Martín" Sanatorio Rosendo García

ENTRE RÍOS

FORMOSA

Hospital de Alta Complejidad “Juan D. Perón" Clínica de Nefrología, Urología y Enfermeda-

UUルY

Sanatorio Nuestra Señora del Rosario

LA RIOJA

Instituto del Corazón (INCOR)

Clinica Privada E.R.I

MENDOZA

Hospital Central

Hospital Españo

Hospital Italiano

Sociedad Española De Socorros Mutuos

Hospital Luis Carlos Lagomaggiore

Hospital Del Carmen

MISIONES

Sanatorio Regional

SANTA FE des Cardiovasculares

Sanatorio Privado San Gerónimo

Sanatorio Médico de Diagnóstico y Tratamiento

Hospital Proviencial "J.M. Cullen"

Instituto Privado de Cardiología"Sagrada

Familia"

Sanatorio Mayo

Sanatorio Santa Fe

Sanatorio San Martín

Sanatorio Nosti

SALTA

Hospital "San Bernardo"

SAN JUAN

Hospital Dr. Guillermo Rawson

Hospital "Dr. Marcial V. Quiroga

Hospital Escuela de Agudos Ramón Madariaga Centro Integral de Medicina de Alta Compleji-

NEUQUÉN

dad (Clinica El Castaño)

Hospital Provincial Neuquén Castro Rendón SANTIAGO DEL ESTERO

RÍO NEGRO

Instituto Cardiovascular Del Sur

Sanatorio Juan XXIII

ROSARIO

Hospital Provincial De Rosario

Hospital Provincial Del Centenario

Sanatorio Los Arroyos

Sanatorio Plaza

Instituto Cardiovascular De Rosario

Hospital De Emergencias Clemente Alvarez

Instituto de Cardiología "González Sabathie"

Centro Materno Infantil Htal Italiano Garibaldi

Hospital Español
Centro Cardiovascular Clinica Yunes

Sanatorio Instituto de Cardiología

TUCUMÁN

Inst. Tucumán de Enfermedades del Corazón Instituto de Cardiología de Tucumán Hospital Centro De Salud Zenon J Santillan

Cardiología del Parque

Sanatorio 9 de Julio

Centro Modelo de Cardiología

Centro Privado de Cardiología

\section{MIEMBROS HONORARIOS}

Dr. Atdemar Álvarez +

Dr. Carlos Benjamín Álvarez

Dr. Roberto Basile

Dr. César Belziti

Dr. Carlos Bertolasi +

Dr. Daniel Boccardo

Dr. Arturo Cagide

Dr. Ramiro Castellanos

Dr. Carlos Crespo

Dr. Miguel Del Río

Dr. Hernán Doval

Dr. René Favaloro +

Dr. Francisco Gadaleta
Dr. Joaquín García
Dr. Hugo Grancelli
Dr. Pablo Heredia
Dr. Juan Humphreys
Dr. Ricardo Iglesias
Dr. Juan Krauss
Dr. Jorge Lerman
Dr. José Martínez Martínez +
Dr. Osvaldo Masoli
Dr. José Milei
Dr. Raúl Oliveri

Dr. Igor Palacios

Dr. Néstor Pérez Baliño

Dr. Horacio Pomes I parraguirre

Dr. Rubén Posse +

Dr. Luis Pozzer

Dr. Osvaldo Robiolo

Dr. César Serra

Dr. Carlos Tajer

Dr. Jorge Trongé

Dr. Alejandro De Cerccio

Dr. Jorge Thierer
COMITÉ ASESOR

Dr. Andrés Ahuad Guerrero

Dr. Raúl J. Bevacqua

Dr. Rafael Cecchi

Dr. Jorge González Zuelgaray

Dr. Hugo Grancelli

Dr. Delfor Hernández

Dr. José Hidalgo

Dr. Gabriel Martino

Dra. Margarita Morley

Dra. Viviana Perugini

\section{EXPRESIDENTES}

1982: Dr. Francisco Gadaleta

1983: Dr. Enrique Retyk

1984: Dr. Ricardo Iglesias

1985: Dr. Juan José Nasif

1986: Dr. Arnaldo Angelino

1987: Dr. Raúl J. Bevacqua

1988: Dr. Andrés Ahuad Guerrero

1989: Dr. Rafael Cecchi

1990: Dra. Viviana Perugini

1991: Dr. Rodolfo Sansalone
1992: Dra. Gladys Aranda 1993: Dr. Gerardo Bozovich

1994: Dr. Alejandro Cherro

1996: Dr. Sergio Baratta

1997: Dr. Félix Paredes

1998: Dr. Marcelo E. Halac

1999: Dr. Pablo Perel

2000: Dr. Esteban Ludueña Clos

2001: Dr. Juan Arellano

2002: Dra. Mariana Pizzella
2003: Dr. Marcelo M. Casas 2004: Dr. Humberto Bassani Molinas 2005: Dr. Martín Descalzo 2006: Dr. Bruno Linetzky 2007: Dr. Juan Cruz López Diez 2008: Dr. Fernando Guardiani 2009: Dr. Diego Lowenstein 2010: Dr. Pablo Pieroni

2011: Dr. Gonzalo Pérez 2012: Dr. Nicolás González
2013: Dr. Ezequiel Zaidel 2014: Dr. Matías Galli

2015: Dr. Luciano Fallabrino

2016: Dr. Darío Igolnikof 


\section{SUMMARY}

REVISTA CONAREC SEPTIEMBRE - OCTUBRE DE 2018 | AÑO 33 | NÚMERO 146

EDITORIAL | EDITORIAL

REPERFUSIÓN EN EL INFARTO AGUDO DE MIOCARDIO CON ELEVACIÓN DEL ST: ES TIEMPO DE ACTUAR

REPERFUSION IN ST SEGMENT ELEVATION MYOCARDIAL INFARCTION: IT IS TIMETO ACT

María A. Spampinato Canals Frau, Javier I. Ortego

REVISIÓN POR EXPERTOS | EXPERT REVIEW

ACERCA DE ESTUDIOS ALEATORIZADOS Y OBSERVACIONALES

ABOUT RANDOMIZED AND OBSERVATIONAL STUDIES

Jorge Thierer

REVISIÓN ANUAL | ANNUAL REVIEW

COMPLICACIONES DE LA VENTILACIÓN MECÁNICA

EN EL POSOPERATORIO DE CIRUGÍA CARDIOVASCULAR

COMPLICATIONS OF MECHANICAL VENTILATION IN POSTOPERATIVE CARE

OF CARDIOVASCULAR SURGERY

Fernando Ríos, Nicolás Diez

MONOGRAFÍA SELECCIONADA I SELECTED MONOGRAPH

HIPERTENSIÓN PULMONAR TROMBOEMBÓLICA CRÓNICA

EN LA ERA DE LA MÍNIMA INVASIVIDAD

CHRONIC THROMBOEMBOLIC PULMONARY HYPERTENSION IN THE ERA

OF MINIMAL INVASIVENESS

Sebastián Srur

ARTÍCULOS ORIGINALES | ORIGINAL ARTICLES

SCORE DE GOLDMAN MODIFICADO PARA LA EVALUACIÓN DE PACIENTES CON DOLOR PRECORDIAL

EN LA GUARDIA CENTRAL

GOLDMAN INDEX MODIFIED FOR THE EVALUATION OF PATIENTS WITH PRECORDIAL PAIN IN THE CENTRAL ER

Carla Vallejos Olmos, Juan P. Ricarte-Bratti, Julio O. Bono, Eduardo Moreyra

CASOS CLÍNICOS | CLINICAL CASES

TROMBOEMBOLISMO PULMONAR RECURRENTE

EN UN PACIENTE CON SÍNDROME ANTIFOSFOLIPÍDICO

Y TROMBOCITOPENIA INDUCIDA POR HEPARINA

RECURRENT PULMONARY THROMBOEMBOLISM IN A PATIENT WITH ANTIPHOSPHOLIPID SYNDROME AND THROMBOCYTOPENIA INDUCED BY HEPARIN

221 Nahuel Litwak, Milagros Seijo

SINDROMEDETAKOTSUBOENPACIENTECONCARDIOPATIA

SÍNDROME DE TAKOTSUBO EN PACIENTE CON CARDIOPATÍA HIPERTRÓFICA

TAKOTSUBO SYNDROME IN PATIENT WITH HYPERTROPHIC CARDIOMYOPATHY Franco Facciuto, Leonardo A. Pilón, Franco L. Marino, Daniela F. Chilabert, Fernando Bagnera, Gerardo Zapata

IMÁGENES EN CARDIOLOGÍA | IMAGES IN CARDIOLOGY

CUANDO EL POSOPERATORIO SE COMPLICA

WHEN THE POSTOPERATIVE PERIOD GETS COMPLICATED

Ignacio A. Vaca Valverde, Rafael Porcile, Ricardo Levin, Juan Fernández, Flavio Salvaggio, Alejandro Botbol

REGLAMENTO DE PUBLICACIONES | RULES OF PUBLICATIONS

ESTRATEGIAS INTRAHOSPITALARIAS DE MEJORA

DEL TIEMPO PUERTA-BALÓN Y EVOLUCIÓN CLÍNICA

EN PACIENTES CON INFARTO AGUDO DE MIOCARDIO

CON SUPRADESNIVEL DEL ST

INTRAHOSPITAL STRATEGIES FOR IMPROVING DOOR-TO-BALLOON TIME

AND CLINICAL EVOLUTION IN PATIENTS WITH ACUTE MYOCARDIAL

INFARCTION WITH ST-SEGMENT ELEVATION

Alan Sigal, Andreína Gil Ramírez, Cristian Garmendia, Noelia Alby,

Lucrecia M. Burgos, Fernando Cura, Alfonsina Candiello 


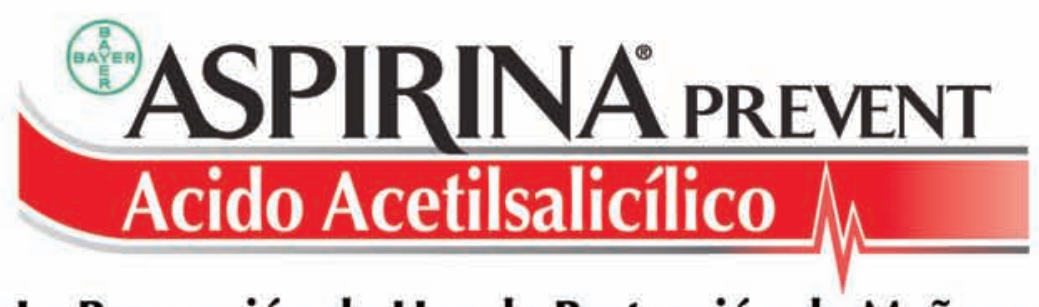

\section{La Prevención de Hoy, la Protección de Mañana}

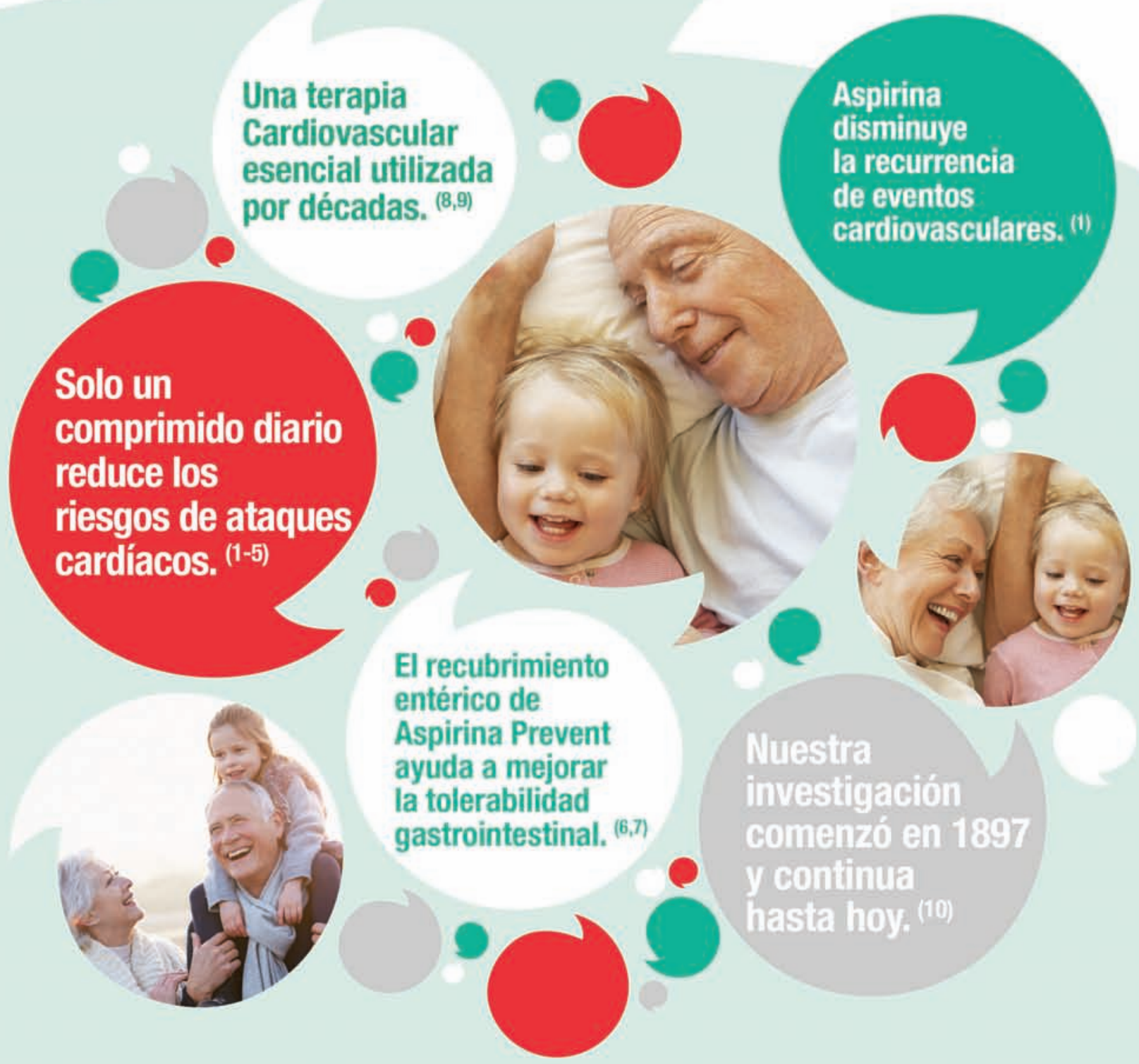

REFERENCLAS: 1. Antithrombotic Trialists' (ATT) Collaboration. Aspirin in the primary and secondary prevention of vascular disease: collaborative meta- analysis of individual participant data from randomised trial. Lancet 2009;373:1849-60. 2. Antithrombotic Trialist' (ATT) Collaboration. Collaborative meta- analysis of randomised trials of antiplatelet therapy for prevention of death, myocardia Infarction, and stroke in high risk patents. BMJ 2002;324 (7329):71-86, 3. BartolucciAA Tendera M and Howard G. Meta- analysys of multiple primary prevention trials of cardiovascular events using aspirin. Am J Cardiol 2011; 107: 1796-801. 4. Ryden L, Frant PJ, Anker SD et al. ESC Guidelines on diabetes, pre-diabetes, and cardiovascular diseases developed in collaboration with the EASD: the Task Force on diabetes, pre- diabetes, and cardiovascular diseases of the European Society of Cardiology (ESC) and developed in collaboration with the European Association for the Study of Diabetes (EASD). Eur Heart 2013; J34: 3035-87. 5. Bayer Aspirin Cardio SmPC. Bayer Healthcare 6. Banoob DW, McCloskey WW, Webster W. Risk of Gastric Iniury with Enteric-Versus Nonnenteric- Coated Aspirin Arn Phat 2013

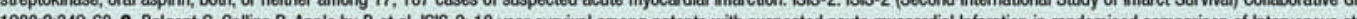
1988;2:349-60. 9. Baigent C, Collins R, Apple by P, et al. ISIS-2: 10 year survival among patents witi suspected acute myocardial Infarction in randomised comparison of Intervenous streptokinase, oral aspirin, both, or neither. The ISS-2 (Second International Study of htarct Survival) Collaborative Group. BMN 1996;316:1337-43. 10. Data on file. Bayer Helthcare. Hitpi//umw.bayercom/enVlelix-hoffmann aspi Last accessed 26/06/14.

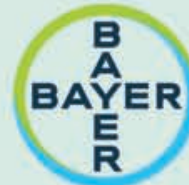


ASPIRINA PREVENT. COMPOSICIÓN Cada comprimido con cubierta entérica contiene: Ácido acetilsalicilico: $100 \mathrm{mg} / 350 \mathrm{mg}$. INDICACIONES 100mg: Reducción del riesgo de mortalidad en pacientes con sospecha de infarto agudo de miocardio. Reducción del riesgo de morbilidad y mortalidad en pacientes con antecedentes de infarto de miocardio. Reducción del riesgo de un primer episodio de infarto de miocardio en pacientes con factores de riesgo cardiovascular, p.ej. diabetes mellitus, hiperlipidemia, hipertensión, obesidad, fumadores, tercera edad. Prevención secundaria de accidentes cerebrovasculares. Reducción de riesgo de ataques isquémicos transitorios (TIA) y accidentes cerebrovasculares en pacientes con TIA. Reducción de riesgo de morbilidad y muerte en pacientes con angina de pecho estable o inestable. Prevención de tromboembolias luego de una cirugia vascular o intervenciones, p.ej. PTCA, endarterectomía carotidea, shunts arteriovenosos. Profilaxis de trombosis venosas profundas y embolias pulmonares luego de inmovilizaciones prolongadas, p.ej. Iuego de cirugias mayores. INDICACIONES $350 \mathrm{mg}$ : Reducción del riesgo de mortalidad en pacientes con sospecha de infarto agudo de miocardio. Reducción del riesgo de morbilidad y mortalidad en pacientes con antecedentes de infarto de miocardio. Reducción del riesgo de un primer episodio de infarto de miocardio en pacientes con factores de riesgo cardiovascular, p.ej. diabetes mellitus, hiperlipidemia, hipertensión, obesidad, fumadores, tercera edad. Prevención secundaria de accidentes cerebrovasculares. Reducción de riesgo de ataques isquémicos transitorios (TIA) y accidentes cerebrovasculares en pacientes con TIA. Reducción de riesgo de morbilidad y muerte en pacientes con angina de pecho estable o inestable. Prevención de tromboembolias luego de una cirugla vascular 0 intervenciones, p.ej.PTCA, endarterectomla carotidea, derivaciones arteriovenosas. Profilaxis de trombosis venosas profundas y embolias pulmonares luego de inmovilizaciones prolongadas, p ej. luego de cirugías mayores. POSOLOGÍA Y FORMA DE ADMINISTRACIÓN $100 \mathrm{mg}$ : Salvo otra indicación médica, se recomienda en adultos la siguiente dosificación. Para reducir el riesgo de mortalidad en pacientes con sospecha de infarto agudo de miocardio: dosis inicial es de 200 a $300 \mathrm{mg}$ administrada tan pronto como se sospeche el IM. En caso de utilizarse en esta indicación, la aspirina con recubrimiento gastrorresistente deberá triturarse o masticarse para lograr una absorción más rápida. Para reducir el riesgo de morbilidad y mortalidad en pacientes con antecedentes de infarto de miocardio; para prevención secundaria de accidentes cerebrovasculares; para reducir el riesgo de ataques isquémicos transitorios (TIA); para reducir el riesgo de morbilidad y muerte en pacientes con angina de pecho estable o inestable; para prevención de tromboembolias luego de una cirugia vascular o intervenciones: 100 a $300 \mathrm{mg}$ por dia. Para reducir el riesgo de un primer episodio de infarto de miocardio en pacientes con factores de riesgo cardiovascular y para profilaxis de trombosis venosas profundas y embolias pulmonares luego de inmovilizaciones prolongadas: 100 a $200 \mathrm{mg}$ por día o $300 \mathrm{mg}$ día por medio. POSOLOGIA Y FORMA DE ADMINISTRACIÓN $350 \mathrm{mg}$ : Salvo otra indicación médica, se recomienda en adultos la siguiente dosificación. Para reducir el riesgo de mortalidad en pacientes con sospecha de infarto agudo de miocardio: 325 mg por día. La dosis de mantenimiento es de $325 \mathrm{mg}$ todos los dias hasta 30 dias posterior al infarto. Luego de los 30 dias deberá tenerse en cuenta la terapia adicional suministrada para evitar un nuevo infarto de miocardio. Para reducir el riesgo de morbilidad y mortalidad en pacientes con antecedentes de infarto de miocardio, para prevención secundaria de accidentes cerebrovasculares; para reducir el riesgo de ataques isquémicos transitorios (TIA); para reducir el riesgo de morbilidad y muerte en pacientes con angina de pecho estable o inestable; para prevención de tromboembolias luego de una cirugia vascular o intervenciones: $325 \mathrm{mg}$ por dia. Para reducir el riesgo de un primer episodio de infarto de miocardio en pacientes con factores de riesgo cardiovascular y para profilaxis de trombosis venosas profundas y embolias pulmonares luego de inmovilizaciones prolongadas: 325 mg dia por medio. CONTRAINDICACIONES. Úlcera gastrointestinal aguda. Diátesis hemorrágica. Hipersensibilidad al ácido acetilsalicílico, a salicilatos, o a algún otro componente de este producto. Antecedentes de asma inducido por la administración de salicilatos o sustancias con una acción similar, en especial drogas antiinflamatorias no esteroideas. Combinación con metotrexate en dosis de $15 \mathrm{mg}$ por semana o más. Último trimestre de embarazo. Falla renal severa. Falla hepática severa. Falla cardiaca severa. ADVERTENCIAS Y PRECAUCIONES. Hipersensibilidad a los analgésicos y a agentes antiinflamatorios/antirreumáticos y en presencia de otras alergias: en pacientes con fiebre de heno, múltiples pólipos nasales, asma bronquial o enfermedades respiratorias crónicas, el ácido acetilsalicilico puede precipitar un broncoespasmo e inducir ataques de asma u otras reacciones de hipersensibilidad. Debido al efecto inhibitorio sobre la agregación plaquetaria que persiste por varios dias luego de su administración, el ácido acetilsalicilico tiende a aumentar las hemorragias durante y luego de intervenciones quirúrgicas (incluyendo cirugías menores, p.ej. extracciones dentales). El paciente debe consultar con su médico acerca del uso de ácido acetilsalicilico. Tratamiento conjunto con anticoagulantes. Algunos AINES, como el ibuprofeno y el naproxeno pueden atenuar el efecto inhibitorio del ácido acetilsalicilico sobre la agregación plaquetaria. El paciente deberá advertir al médico cuando esté bajo terapia con ácido acetilsalicilico y deba tomar ibuprofeno. En pacientes que sufren de graves deficiencias de la glucosa-S-fosfato deshidrogenasa (G6PD), el ácido acetilsalicilico puede inducir hemólisis o anemia hemolitica. Los factores que pueden aumentar el riesgo de hemólisis son, por ejemplo dosis altas, fiebre o infecciones agudas. A dosis bajas, el ácido acetilsalicilico reduce la excreción del ácido úrico. Esto puede favorecer la aparición de gota en pacientes predispuestos. Antecedentes de úlcera gastrointestinal incluyendo úlceras crónicas o recurrentes o antecedentes de hemorragias gastrointestinales. Daño de función renal y hepática. Los pacientes con insuficiencia renal o en pacientes con trastornos en la circulación cardiovascular el ácido acetilsalicilico pueden aumentar el riesgo de insuficiencia renal y falla renal aguda. Alteración de la función hepática. Los productos medicinales que contengan ácido acetilsalicilico/aspirina no deben ser utilizados en nin̂os y adolescentes menores de 16 años que tengan enfermedades virales, con o sin fiebre sin consultar al médico. Existe el riesgo de que ciertas enfermedades virales, como ser influenza A, B y varicela provocar el sindrome de Reyé, una patologia rara pero severa que requiere una intervención médica inmediata. REACCIONES ADVERSAS. Las reacciones adversas a medicamentos (RAM) enumeradas a continuación se basan en informes espontáneos postcomercialización con todas las formulaciones de aspirina de administración oral, incluyendo tratamientos a corto y largo plazo, por lo que una clasificación de acuerdo con las categorias CIOMS III de frecuencia no es pertinente: trastornos del sistema inmunitario: reacciones de hipersensibilidad con las respectivas manifestaciones clinicas y de laboratorio: sindrome de asma, reacciones leves a moderadas que puedan afectar a la piel, vias respiratorias, tracto gastrointestinal y sistema cardiovascular, incluyendo sintomas como ser sarpullido, urticaria, edema, prurito, rinitis, congestión nasal, insuficiencia cardiorrespiratoria y muy raramente reacciones severas, incluyendo shock anafiláctico; trastornos gastrointestinales: trastornos frecuentes del tracto gastrointestinal bajo y alto, asi como signos y sintomas de dispepsia, dolor abdominal y gastrointestinal, raramente trastornos como ser: inflamación gastrointestinal, úlcera gastrointestinal que puede evolucionar muy raramente a una úlcera gastrointestinal sangrante y perforación con los correspondientes valores de laboratorio y los caracteristicos signos clinicos y sintomas. Trastornos hepatobiliares: se han reportado casos aislados y transitorios de alteraciones de la función hepática. Trastornos de la sangre y del sistema linfático: debido al efecto inhibitorio sobre la agregación plaquetaria, el ácido acetilsalicilico puede ser asociado con un riesgo aumentado de sangrados. Se han observado sangrados, como ser hemorragias perioperatorias, hematomas, epistaxis, sangrados urogenitales y gingivales. Se han informado episodios raros a muy raros de sangrados severos, como ser hemorragia del tracto gastrointestinal, hemorragia cerebral (especialmente en pacientes con hipertensión no controlada y/o con administración concomitante de agentes anticoagulantes) que pueden ser, en casos aislados, fatales. Las hemorragias pueden provocar anemias agudas y crónicas secundarias a hemorragias/anemia ferropénica con los correspondientes valores de laboratorio los caracteristicos signos clinicos y sintomas, tales como astenia, palidez, hipoperfusión. Se ha reportado hemólisis y anemia hemolitica en pacientes con formas graves de deficiencia de glucosa-6-fosfato (G6PD). Trastornos del sistema nervioso: mareos y tinnitus son signos indicativos de sobredosis. Trastornos renales y urinarios: se han reportado casos de alteración de la función renal e insuficiencia renal aguda. PRESENTACIón Envases con 4,10,20,30,50 y 100 comprimidos.

Bayer S.A., Ricardo Gutiérrez 3652, (B1605EHD), Munro, Bs. As., Argentina.

Director Técnico: José Luis Role, Farmacéutico.

Especialidad medicinal autorizada por el Ministerio de Salud. Certificado N ${ }^{\circ} 33575$ Versión: CCDS v6 revisión: 09.2017 


\title{
SUMARIO ANALITICO
}

\author{
ANALYTICAL SUMMARY
}

\section{REVISIÓN POR EXPERTOS | EXPERT REVIEW}

215

\section{ACERCA DE ESTUDIOS ALEATORIZADOS Y OBSERVACIONALES JORGETHIERER}

Cuando se gradúa la fuerza de evidencia para formular una recomendación médica, se admite que los estudios aleatorizados tienen preeminencia sobre los observacionales. Los estudios aleatorizados eliminan el sesgo de selección y aseguran la distribución similar de las variables basales conocidas y desconocidas entre la rama de tratamiento activo y el control o comparador. Pero la validez externa de los resultados suele ser cuestionada por ser los pacientes, los médicos y los procedimientos poco representativos de la realidad. Los estudios observacionales tienen en cambio alta validez externa, pero están sujetos a gran cantidad de sesgos y el efecto de confundidores. Hay métodos estadísticos que pueden remediar solo en parte este déficit de los estudios observacionales. La realización de estudios aleatorizados basados en registros, y más pragmáticos a la hora de definir participantes e in tervenciones, puede contribuir a salvar la distancia entre uno y otro tipo de estudios. La decisión de obtener la información más pura y menos sesgada, información fidedigna y representativa, es el paso inicial para avanzar en el camino del conocimiento.

REVISIÓN ANUAL | ANNUAL REVIEW

\section{COMPLICACIONES DE LA VENTILACIÓN MECÁNICA EN EL POSOPERATORIO DE CIRUGÍA CARDIOVASCULAR}

FERNANDO RÍOS, NICOLÁS DIEZ

La cirugía cardíaca conlleva una variedad de complicaciones pulmonares, al gunas de las cuales contribuyen a la lesión pulmonar o síndrome de dificultad respiratoria aguda, y se asocia con aumento de la permeabilidad vascula pulmonar, el incremento del agua pulmonar extravascular y/o la generación de atelectasias. Los mecanismos implicados en el desarrollo de estas incluyen dos causas fundamentales: el trauma del procedimiento quirúrgico y el efecto de la ventilación mecánica sobre un entorno inflamatorio. Esta revisión se enfocará en abordar las diferentes efectos cardiovaculares y complicaciones de la ventilación mecánica en pacientes en el posoperatorio de cirugía cardíaca, y además se revisarán diferentes estrategias terapéuticas para minimizar la lesión pulmonar.

\section{MONOGRAFÍA SELECCIONADA I SELECTED MONOGRAPH}

\section{HIPERTENSIÓN PULMONAR TROMBOEMBÓLICA CRÓNICA EN LA ERA DE LA MÍNIMA INVASIVIDAD \\ SEBASTIÁN SRUR}

La hipertensión pulmonar tromboembólica crónica (HPTC) es un tipo de hipertensión pulmonar que se caracteriza por la estenosis de los vasos pulmonares con material trombótico de resolución incompleta, así como por un grado variable de arteriopatía esclerofibrótica. La importancia de su correcto y precoz diagnóstico radica en que posee un tratamiento potencialmente curativo, la tromboendarterectomía pulmonar. Sin embargo un tercio de los pacientes no se operan por el elevado riesgo del procedimiento o porque el proceso tromboembólico resulta técnicamente inoperable; es en este grupo de pacientes en que cobran importancia otras alternativas terapéuticas, como la angioplastia con balón a las arterias pulmonares y el tratamiento farmacológico dirigido. Tanto la angioplastia con balón como las terapias farmacológicas resultan alternativas prometedoras. Nuevas investigaciones son fundamentales para definir mejor el rol de éstas en la HPTC

\section{ARTÍCULOS ORIGINALES | ORIGINAL ARTICLES}

\section{ESTRATEGIAS INTRAHOSPITALARIAS DE MEJORA DEL TIEMPO PUERTA-BALÓN Y EVOLUCIÓN CLÍNICA EN PACIENTES CON INFARTO AGUDO DE MIOCARDIO CON SUPRADESNIVEL DEL ST}

ALAN SIGAL, ANDREÍNA GIL RAMÍREZ, CRISTIAN GARMENDIA, NOELIA ALBY, LUCRECIA M. BURGOS, FERNANDO CURA, ALFONSINA CANDIELLO

Introducción. El tratamiento exitoso del infarto con supradesnivel del ST (IAMCEST) es tiempo dependiente. Existe limitada información en nuestro país acerca de la efectividad de estrategias en la reducción de los tiempos de isquemia. E objetivo de este trabajo es analizar el impacto de la aplicación de estrategias intrahospitalarias de mejora (EIHM) en la reducción del tiempo puerta balón (TPB), y en la evolución clínica de pacientes con IAMCEST.

Métodos. Estudio observacional retrospectivo sobre una base de datos prospec tiva desde el año 2014 hasta 2017. Se incluyeron de forma consecutiva pacientes con IAMCEST de $<12$ horas de evolución, que ingresaron para angioplastia primaria; y se compararon según el período: pre- (2014-2015) o posimplementación de EIHM (2016-2017). Las EIHM evaluadas fueron: preactivación de hemodinamia y bypass de guardia. Como punto final primario se analizó el TPB. Como puntos secundarios se evaluó mortalidad a 180 días, reinfarto y nueva revascularización.

Resultados. Se analizaron 155 pacientes, 40,6\% dentro del grupo pre-EIHM y $59,4 \%$ en el grupo post-EIHM. El $67 \%$ llegó en ambulancia y el restante por sus propios medios. No existieron diferencias respecto al tratamiento médico instaurado (74\% recibió ticagrelor). Se observó una reducción del TPB en el grupo post-EIHM en comparación al preimplementación, de 42 min (rango intercuartílico 25-75 [RIC]: 30-55,5) vs. 55 min (RIC: $32-77$ ), $p=0.014$, y en el tiempo total de isquemia, de 161,5 min (RIC: 120-239,5) vs. $197 \mathrm{~min}(145-277), p=0,02$. No se encontró diferencia significativa en mortalidad intrahospitalaria ( $3,1 \mathrm{vs}, 2,1 \%$; $p=0,7$ ) ni en los eventos clínicos. El seguimiento se realizó en el 94,2\% de los pacientes, con una mediana de 7 meses (RIC: 1,23-15,7). A los 180 días no hubo diferencias en el punto final combinado (27,3 grupo pre- vs. $15,3 \%$ post-EIHM; log rank test $\mathrm{p}=0,33)$. En el modelo de regresión de Cox, la ElHM tuvo un hazard ratio de 0,69 (intervalo de confianza 95\%: 0,32-1,47 $p=0,34$ ).

Conclusión. Las EIHM implementadas lograron disminuir de forma significativa el TPB al igual que el tiempo total de isquemia, indicadores de la calidad del centro y del pronóstico intrahospitalario. Se observó una tendencia a la disminución de evento combinado en el seguimiento, aunque de forma no significativa.

\section{TROMBOEMBOLISMO PULMONAR AGUDO EN LA REPÚBLICA} ARGENTINA: PROTOCOLO DEL REGISTRO CONAREC XX

IGNACIO M. CIGALINI, CRISTHIAN E. SCATULARO, JUAN C. JÁUREGUI, MAICO I. BERNAL, JAVIER I. ORTEGO, JUAN M. ABOY, DANIEL CORNEJO, SEBASTIÁN GARCÍA ZAMORA, EZEQUIEL J. ZAIDEL

Introducción: El tromboembolismo de pulmón agudo (TEP) representa la tercera causa de mortalidad cardiovascular. Sin embargo, existen pocos datos de esta patología en nuestro país.

Objetivo: Describir las características basales, evolución y tratamiento implementado en pacientes internados por TEP agudo en Argentina.

Métodos: Estudio multicéntrico, prospectivo y observacional de pacientes con diagnóstico de TEP agudo internados en centros con residencia de Cardiología desde octubre de 2016 a noviembre de 2017 independientemente si este fuere causa de la internación o surgiera como complicación de hospitalización por otra causa. Se remitirán datos en forma online a través de un sitio seguro con contraseña individual para cada centro participante. Se analizarán variables sociodemográficas, clínicas, estudios complementarios y evolución intrahospitalaria. Se realizará auditoría cruzada al 20\% de los centros.

Conclusiones: El registro CONAREC XX aportará valiosa información para conocer la realidad del TEP agudo en nuestro país. 
SCORE DE GOLDMAN MODIFICADO PARA LA EVALUACIÓN DE PACIENTES CON DOLOR PRECORDIAL

\section{EN LA GUARDIA CENTRAL}

CARLA VALLEJOS OLMOS, JUAN P. RICARTE-BRATTI, JULIO O. BONO, EDUARDO MOREYRA

Introducción. El score de Goldman modificado ha demostrado efectividad en aquellos pacientes que consultan a la guardia por dolor precordial. A pesar de ello, sigue siendo poco usado como herramienta para el alta precoz en combinación con una sola muestra troponina T ultrasensible (TnT US)

Objetivo. Evaluar la especificidad, sensibilidad, el valor predictivo negativo (VPN) y el valor predictivo positivo (VPP) del score de Goldman modificado y de la TnT US al ingreso.

Materiales y métodos. Estudio prospectivo descriptivo de pacientes que acudieron por guardia central con dolor precordial a quienes se les realizó electrocardiograma, dosaje de TnT US y se evaluó el riesgo de enfermedad coronaria a través del puntaje de riesgo de Goldman modificado para establecer un protocolo de alta segura y precoz. Se realizó un seguimiento de los pacientes a los 30 días del alta.

Resultados. Se incluyeron 34 pacientes. El score de Goldman modificado fue aplicado a sujetos con dolor precordial con sospecha de síndrome coronario agudo (SCA), con una sensibilidad del 100\%, especificidad del $41 \%$, VPP $41 \%$ y VPN 100\% para realizar el diagnóstico rápido de exclusión. Para la TnT US hora 0, la sensibilidad y especificidad para SCA fueron del $27 \%$ y del $79 \%$, respectivamente $(p=0,001)$.

Conclusión. Este protocolo incorpora un score de evaluación de riesgo estructurado de manera simple con parámetros clínicos y dosaje de troponina T ultrasensible, tiene el potencial de permitir el alta temprana de los pacientes con sospecha de síndrome coronario agudo, y la internación precoz en los que se diagnosticó. Solo en un escaso número de pacientes se debieron realizar otros estudios para aclarar el diagnóstico.

\section{CASOS CLÍNICOS | CLINICAL CASES}

TROMBOEMBOLISMO PULMONAR RECURRENTE EN UN PACIENTE CON SÍNDROME ANTIFOSFOLIPÍDICO Y TROMBOCITOPENIA INDUCIDA POR HEPARINA

\author{
NAHUEL LITWAK, MILAGROS SEIJO
}

El tromboembolismo pulmonar es una urgencia cardiovascular de pronóstico variable y en su estudio etiológico debemos buscar los factores que puedan influir en su génesis y precipitar resultados adversos. La trombocitopenia inducida por heparina y el síndrome antifosfolipídico son enfermedades hematológicas que pueden participar en la fisiopatogenia del tromboembolismo pulmonar y complicar su evolución. A continuación presentamos un caso caso demuestra la ocurrencia de ambas entidades en un paciente.

\section{SÍNDROME DE TAKOTSUBO EN PACIENTE CON CARDIOPATÍA} HIPERTRÓFICA

FRANCO FACCIUTO, LEONARDO A. PILÓN, FRANCO L. MARINO,

DANIELA F. CHILABERT, FERNANDO BAGNERA, GERARDO ZAPATA

La cardiomiopatía de takotsubo (CT) es una condición aguda y reversible, carac terizada por una disfunción ventricular principalmente de los segmentos apicales del ventrículo izquierdo (VI), con hipercontractilidad de los basales. Esto puede generar en ciertas ocasiones una obstrucción del tracto de salida del VI, Io que se puede exacerbar aún más en pacientes con cardiopatías hipertróficas. Debido a que la combinación de estas dos entidades es un escenario muy poco frecuente y a que su comportamiento en agudo muchas veces no es del todo benigno, su manejo puede ser extremadamente desafiante.

Se presenta el caso de un paciente de 72 años con hipertrofia ventricular izquierda y CT que evoluciona con shock cardiogénico.

IMÁGENES EN CARDIOLOGÍA IMAGES IN CARDIOLOGY

\section{CUANDO EL POSOPERATORIO SE COMPLICA}

FLAVIO SALVAGGIO, ALEJANDRO BOTBOL

El pseudoaneurisma aórtico (PSA) es una complicación reportada después de una cirugía cardíaca. La seguridad y eficacia de las terapias endovasculares para esta patología aún es controvertida. Sin embargo, el tratamiento quirúrgico de Ios PSA se asocia con una alta mortalidad y morbilidad, y en algunos casos no es factible, por lo que el cierre percutáneo se ha descrito como una alternativa. Presentamos un caso de tratamiento endovascular de PSA después de reemplazo de válvula aórtica. 


\section{- DIOCAM}

\section{La vida esta ahi, simple y tranquila...}

Paul Verlaine

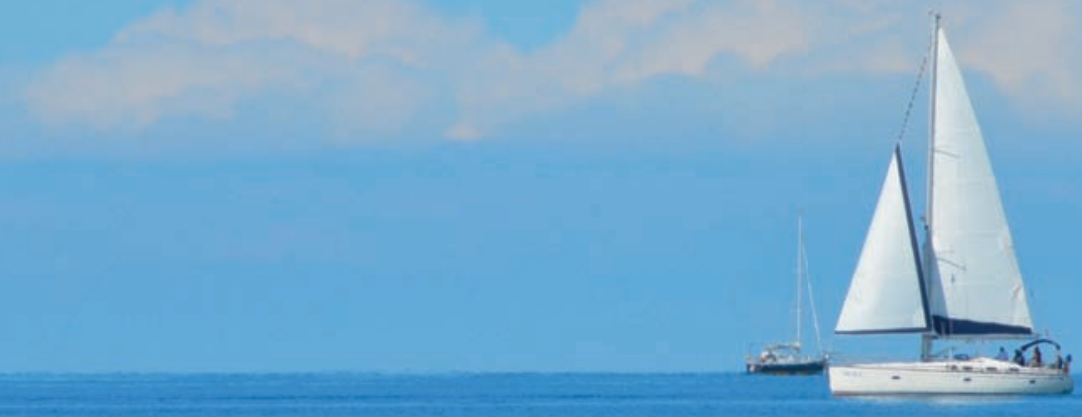

\section{PRESENTACIONES}

DIOCAM SL 0,25 MG:

envase de 30 comprimidos sublinguales

DIOCAM 0,5 - 1 - 2 MG:

envases de 30 y 60 comprimidos

\section{(11)}

\section{DIOCAM 2 \\ CLONAZEPAM $2 \mathrm{mg}$}

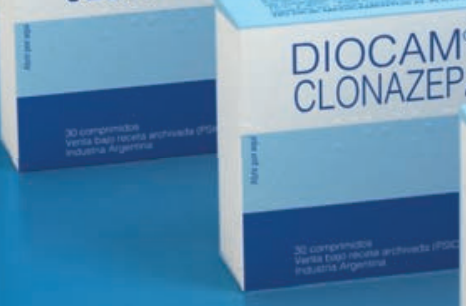

\section{$\mathrm{DIOCAM}^{\oplus 1}$ \\ CLONAZEPAM $1 \mathrm{mg}$}

\section{DIOCAM ${ }^{\circ} 0,5$ \\ CLONAZEPAM $0,5 \mathrm{mg}$}
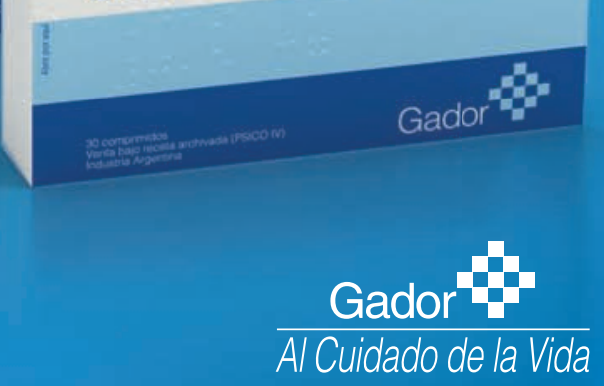


\title{
REPERFUSIÓN EN EL INFARTO AGUDO DE MIOCARDIO CON ELEVACIÓN DEL ST: ES TIEMPO DE ACTUAR
}

\section{REPERFUSION IN ST SEGMENT ELEVATION MYOCARDIAL INFARCTION: IT IS TIMETO ACT}

\author{
REVISTA CONAREC 2018;33(146):213-214 | DOI:10.32407/RCON/2018146/0213-0214
}

Las enfermedades cardiovasculares son la principal causa de muerte a nivel mundial. Nuestro país no está fuera de la estadística, con un porcentaje cercano al 40\%. Dentro de este porcentaje cumple un papel protagónico el infarto agudo de miocardio $(\text { IAM) })^{1,2}$.

Ya desde principios del siglo XIX se conocía que la trombosis coronaria es la causa del IAM. Pero no fue hasta el siglo XX que surgieron las primeras publicaciones acerca del tratamiento de la enfermedad y la importancia de la restauración de la circulación coronaria, señalando Herrick que "la esperanza para el miocardio dañado radica en asegurar un suministro de sangre a través de los vasos sanguíneos vecinos para restaurar en la medida de lo posible su integridad funcional". Años después, este mismo autor adaptó el electrocardiograma al reconocimiento del IAM. Eran los inicios del diagnóstico del Infarto agudo de miocardio con supradesnivel del $\mathrm{ST}^{3}$.

El tratamiento de esta entidad fue creciendo a pasos agigantados hasta que, gracias a grandes avances tecnológicos, se logró en 1977 llevar a cabo la primera angioplastia coronaria con balón y en 1986 la implantación del primer stent 4.5 .

En la actualidad, uno de los principales desafíos, junto con el desarrollo de programas de promoción y prevención de la enfermedad cardiovascular, es la implementación de estrategias de acción que nos lleven a lograr, gracias al trabajo en equipo, la reducción de los tiempos necesarios para que el paciente reciba un tratamiento óptimo.

El artículo publicado en este número de nuestra revista es un ejemplo de cómo los residentes en conjunto con diferentes servicios del hospital podemos trabajar en pos de mejorar los tiempos de reperfusión de los pacientes que se atienden en nuestros centros.

En los últimos años, iniciativas como el ARGEN-IAM-ST o Stent save a life han comenzado a generar grupos de trabajo dedicados específicamente a identificar barreras y trabajar en ellas para lograr reducir los tiempos de reperfusión del infarto. El ejemplo más significativo es el Sistema en Red Integrada de los Hospitales Públicos de la ciudad de Rosario, Argentina ${ }^{6-9}$.

En el mencionado artículo "Estrategias Intrahospitalarias de mejora del tiempo puerta balón y evolución clínica en pacientes con Infarto Agudo de Miocardio con supradesnivel del ST" demuestra que la implementación de sistemas en red, con protocolos organizados de actuación pueden lograr acercarnos a los objetivos de tiempo de reperfusión estipulados a nivel mundial ${ }^{10}$. Interesante resulta que, si bien no fue estadísticamente significativa, se observó una reducción en el objetivo compuesto de mortalidad, reinfarto y nueva revascularización a los 180 días. Esto nos plantea el interrogante acerca de qué sucederá con esos pacientes a 1, 2 o 5 años.

Además, sería interesante valorar otros puntos que quizás al momento de la evaluación en la guardia no tomamos en cuenta, como la clase funcional y disfunción ventricular a largo plazo, años de vida ajustados por discapacidad, y gastos en salud públi$\mathrm{ca}$, entre otros.

Como residentes de Cardiología, y futuros cardiólogos, debemos seguir el ejemplo de este grupo de residentes y comprometernos en la monitorización de los tiempos de reperfusión para luego a través de su análisis plantear nuevas estrategias que puedan reducirlos. También cabe destacar que es primordial la implementación de programas de prevención cardiovascular y la formación de los ciudadanos para hacerlos partícipes y responsables de su salud.

MARÍA A. SPAMPINATO CANALS FRAU Residente de Cardiología Clínica. Hospital Central de Mendoza, Rep. Argentina. agustinaspampinato@gmail.com 


\section{BIBLIOGRAFÍA}

1. OPS/OMS, Situación de Salud en las Américas: Indicadores Básicos 2013. Washington, D.C. Estados Unidos de América, 2013.

2. Benjamin EJ, Blaha MJ, Chiuve SE, Cushman M, Das SR, Deo R, et al. Heart Disease and Stroke Statistics-2017 Update: A Report From the American Heart Association. Circulation 2017;135(10):e146-e603.

3. Sarmento-Leite R, Krepsky AM, Gottschall CA. Acute myocardial infarction. One century of history. Arq Bras Cardiol 2001;77(6):593-610.

4. Braunwald E. The treatment of acute myocardial infarction: the Past, the Present and the Future. Eur Heart J Acute Cardiovasc Care 2012;1(1):9-12.

5. Tomberli B, Mattesini A, lacopo Baldereschi G, Di Mario C. Breve historia de los stents coronarios. Rev Esp Cardiol 2018;71(5):312-9.

6. Calenta CH, Manavella BD, Mas LR, Arias LM, Najenson M, Zangroniz PD. Código Infarto Rosario. Impacto de un modelo en red integrada en salud pública para la atención del paciente con infarto agudo de miocardio con elevación del ST. Rev Fed Arg Cardiol 2016;45(4):174-8.
7. Gagliardi JA, Charask A, Perna E, D'imperio H, Bono J, Castillo Costa Y, et al. Encuesta nacional de infarto agudo de miocardio con elevación del ST en la República Argentina (ARGEN-IAM-ST). Rev Argent Cardiol 2016;84:548-57.

8. Candiello A, Cigalini IM, Burgos L, Ortego Jl, García Zamora S, Godoy Armando C. Hoja de ruta en el manejo del infarto agudo de miocardio con elevación del segmento ST. Revista CONAREC 2017;33(138):13-4.

9. Ortego J, Aveni S, Ripa L, Estrella N, Suriano J, Ferraro M, et al. Análisis de tiempos de intento de reperfusión en pacientes con infarto con elevación de ST. Revista CONAREC 2016;32(137):274-8.

10. Ibanez B, James S, Agewall S, Antunes MJ, Bucciarelli-Ducci C, Bueno H, et al. 2017 ESC Guidelines for the management of acute myocardial in farction in patients presenting with ST-segment elevation: The Task Force for the management of acute myocardial infarction in patients presenting with ST-segment elevation of the European Society of Cardiology (ESC). Eur Heart J 2018;39(2):119-77. 


\title{
ACERCA DE ESTUDIOS ALEATORIZADOS Y OBSERVACIONALES
}

\author{
ABOUT RANDOMIZED AND OBSERVATIONAL STUDIES
}

\author{
JORGE THIERER ${ }^{1}$
}

\section{RESUMEN}

Cuando se gradúa la fuerza de evidencia para formular una recomendación médica, se admite que los estudios aleatorizados tienen preeminencia sobre los observacionales. Los estudios aleatorizados eliminan el sesgo de selección y aseguran la distribución similar de las variables basales conocidas y desconocidas entre la rama de tratamiento activo y el control o comparador. Pero la validez externa de los resultados suele ser cuestionada por ser los pacientes, los médicos y los procedimientos poco representativos de la realidad. Los estudios observacionales tienen en cambio alta validez externa, pero están sujetos a gran cantidad de sesgos y el efecto de confundidores. Hay métodos estadísticos que pueden remediar solo en parte este déficit de los estudios observacionales. La realización de estudios aleatorizados basados en registros, y más pragmáticos a la hora de definir participantes e intervenciones, puede contribuir a salvar la distancia entre uno y otro tipo de estudios. La decisión de obtener la información más pura y menos sesgada, información fidedigna y representativa, es el paso inicial para avanzar en el camino del conocimiento.

Palabras clave: medicina basada en la evidencia; ensayo clínico controlado aleatorio; estudios observacionales.

\begin{abstract}
When strength of evidence is graduated to formulate a medical recommendation, it is admitted that randomized studies have pre-eminence over observational ones. Randomized studies eliminate selection bias and ensure a similar distribution of known and unknown baseline variables between the active treatment arm and the control or comparator. But the external validity of the results is often questioned because patients, doctors and procedures are not very representative of reality. Observational studies have high external validity, but are subject to a large number of biases and the effect of confounders. There are statistical methods that can remedy only partially this deficit of observational studies. The fulfilment of randomized studies based on registries, that are more pragmatic when defining participants and interventions, can help to bridge the gap between one type of study and another. The decision to obtain the purest and least biased information, reliable and representative information, is the initial step to advance in the path of knowledge
\end{abstract}

Keywords: evidence-based medicine; randomized controlled clinical trial; observational studies.

REVISTA CONAREC 2018;33(146):215-219| DOI:10.32407/RCON/2018146/0215-0219

\section{INTRODUCCIÓN}

Cuando se clasifica la evidencia sobre la que se formulan recomendaciones en las guías de práctica clínica, la mayor fuerza de evidencia, rotulada como A, es la que corresponde a datos derivados de al menos 2 estudios aleatorizados o metaanálisis. La evidencia B es la que se desprende de un solo estudio aleatorizado o estudios no aleatorizados de grandes dimensiones (cohorte, caso control), y la C la que corresponde a consenso de opinión de expertos, reporte de casos o estándar de cuidado. Está instalada, entonces, ya desde la clasificación, la preeminencia de los estudios de intervención aleatorizados sobre los estudios observacionales.

\section{ESTUDIOS ALEATORIZADOS}

Cuando comparamos una intervención determinada (estrategia diagnóstica, droga, procedimiento invasivo, etc.) con un control, un placebo o la ausencia de intervención, si somos nosotros quienes decidimos quién recibirá cada intervención, seguramente los elegidos ten1. Jefe de la Unidad de Insuficiencia Cardíaca. Centro de Educación Médica e
Investigaciones Clínicas"Norberto Quirno" (CEMIC).

$\triangle$ Correspondencia: Dr. Jorge Thierer. Valdenegro 4337, CP C1430EFA CABA, Rep. Argentina.Email:sinuhe63@yahoo.com

Los autores declaran no tener conflictos de intereses.

Recibido: 01/10/2018| Aceptado: 05/11/2018 drán características que los diferencien de aquellos en quienes decidimos no intervenir. Consciente o inconscientemente es casi seguro que seleccionaremos para la intervención aquellos en quienes juzgamos más factible que la misma sea exitosa. A la hora de decir, en base a los resultados, si la intervención es útil o no, lógicamente surgirá la duda de si lo que sucede se debe a la intervención, o a las características diferentes de los intervenidos y los no intervenidos. Sin duda, la gran ventaja de los estudios aleatorizados radica en que justamente la asignación aleatoria de las intervenciones asegura la distribución balanceada de las características basales conocidas y desconocidas, y elimina el sesgo de selección. Si se demuestra un efecto estadísticamente significativo de la nueva intervención respecto de su comparador (placebo u otra intervención), el mismo podrá ser atribuido a esta y no a otros factores (confundidores), que por estar desigualmente repartidos entre ambas ramas pueden ser los verdaderos responsables de la diferente evolución'. La Figura 1 ilustra la relación entre intervención, confundidor y evento. La Figura 2, un ejemplo: el empleo de anticoagulantes orales (ACO) en pacientes con fibrilación auricular asegura menor incidencia de accidente cerebrovascular (ACV). Pero si indicamos ACO a pacientes con tensión arterial normal, y por lo tanto con menos riesgo de ACV, no sabremos a qué atribuir esta menor incidencia.

Además de eliminar el sesgo de selección, los estudios aleatorizados tienen una serie de ventajas: diseño y análisis rigurosos; en muchos casos la instauración del ciego (doble ciego, simple ciego, e incluso la adjudicación ciega de eventos por personal no involucrado en las acciones cotidianas del estudio) que evita el sesgo de observación; el control (con placebo, cuando es posible, para descartar el efecto pla- 


\section{CONFUNDIDORES}

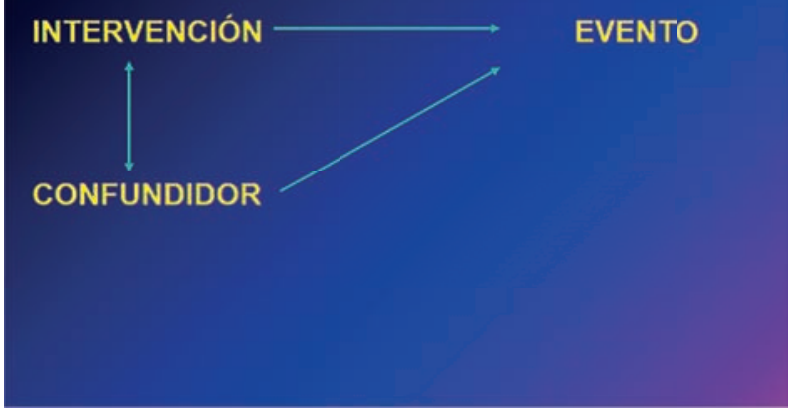

Figura 1. Relación entre intervención, confundidor y evento.

cebo que toda intervención acarrea, y si no lo es, con un control preferentemente concurrente); y el seguimiento estrecho de todos los incluidos para evitar pérdida de pacientes y por ende el sesgo de seguimiento diferencial ${ }^{2}$.

Pero es justo reconocer en los estudios aleatorizados un conjunto de "puntos débiles". Por empezar, las instituciones, médicos y pacientes que participan de los mismos tienen la voluntad de participar y aceptan el proceso de asignación aleatoria y los controles frecuentes. No son por lo tanto similares a aquellos que por diferentes razones no lo hacen (limitaciones por disponibilidad de tiempo o cercanía, deseo tanto del médico como del paciente de instaurar o no instaurar un tratamiento determinado). Y a ello se suma que en general los estudios aleatorizados tienen criterios de inclusión y exclusión estrictos, razón por la cual el grueso de los pacientes considerados inicialmente para participar terminan no haciéndolo. De allí que son estudios de baja validez externa. Hay por otra parte un presupuesto al que deben ceñirse, lo cual impone limitaciones económicas y de duración en el seguimiento. Y, por último, no son útiles para definir la incidencia de eventos o efectos adversos muy poco frecuentes ${ }^{3}$.

Algunas dudas surgen naturalmente al examinar la lógica de implementación de los estudios aleatorizados. Es cierto sí que la aleatorización elimina el sesgo de selección. Pero no es menos cierto es que se selecciona la población que participará del ensayo. Y esa población seleccionada es sin duda aquella donde la intervención tiene más probabilidad de demostrar su efecto beneficioso, si lo hubiera. Procedimientos de control y seguimiento estrecho, pacientes que acuden a todas las visitas, y libres de comorbilidades que amenacen la evolución y el cumplimiento más allá de la patología de interés, análisis frecuentes, etc. Los centros, procedimientos, médicos y pacientes de la vida diaria en general no aseguran tanta perfección.

Un instrumento que puede ser útil a la hora de juzgar la validez externa de un estudio es el PRECIS (pragmatic-explanatory continuum indicator summary $)^{4}$. Considera una serie de ejes que deben ser valorados a la hora de definir dicha validez: la selección de los participantes, la flexibilidad con la que se aplica la intervención, la selección de los médicos que la aplican, cuál es el comparador usual, cómo se seleccionan los médicos que aplican el comparador, si hay visitas de seguimiento

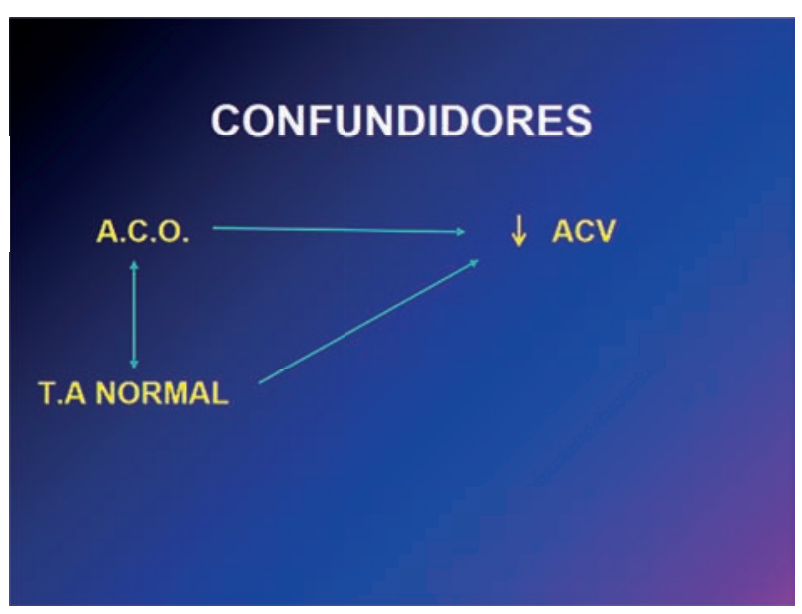

Figura 2. Ejemplo de confundidor. La descripción de la figura está en el texto. ACO: anticoagulantes orales. ACV: accidente cerebrovascular. TA: tensión arterial.

formales, cómo y quién adjudica el punto final, si se mide o no la adherencia de los pacientes y los médicos, y si todos los pacientes son analizados. Cuanto más se parece un estudio a la práctica cotidiana, se dice que es más pragmático. Si por el contrario se caracteriza por intervenciones y mediciones muy estrictas, que lo asemejan más a un ensayo de laboratorio, se dice que es un estudio explicativo. Cuanto más explicativo, seguramente más validez interna y menos validez externa. La Figura 3 ilustra gráficamente el instrumento. Cada semieje representa una de las características citadas. Sobre el mismo se coloca un punto que cuanto más cercano al centro implica un control más estricto y mayor exigencia. Se unen los puntos entre sí y se delimita de esta manera un polígono. Cuanto mayor la superficie que cubre, más pragmático el estudio. Cuanto menor, más explicativo. Recientemente hemos conocido una actualización de este instrumento, el PRECIS $2^{5}$.

La postura oficial es que un ensayo aleatorizado parte a priori de un nivel de evidencia alto. El instrumento GRADE plantea sin embargo una serie de puntos que deben ser tenidos en cuenta a la hora de juzgar la calidad de la evidencia. La falta de ocultamiento a la hora de asignar la intervención, la falta de ciego, el reporte selectivo de eventos, la pérdida en el seguimiento, la detención adelantada del estudio, son todas condiciones que disminuyen la fuerza de la evidencia. La inconsistencia o heterogeneidad en los hallazgos (efectos notables para algún punto final y no para otros, o muy diferente fuerza de asociación con diferentes resultados), la imprecisión en los resultados (intervalos de confianza que incluyen el 1 entre los valores posibles de un RR o un OR, o que incluyen el 0 cuando nos referimos a la diferencia en una variable continua) son otros criterios que restan credibilidad a las conclusiones ${ }^{6}$.

\section{ESTUDIOS OBSERVACIONALES}

Los estudios observacionales, en los que se comparan pacientes efectivamente tratados de diferente manera, pero en los que la indicación no ha sido aleatoria, merecen, como ya dijimos, una serie de críticas. Son estudios sujetos a gran cantidad de sesgos: sesgo de selección, de observación e inmortalidad (para empezar a recibir una medicación es necesario estar vivo; si se adjudica a los que reciben dicha intervención el tiempo desde que ingresaron a una cohorte hasta que 


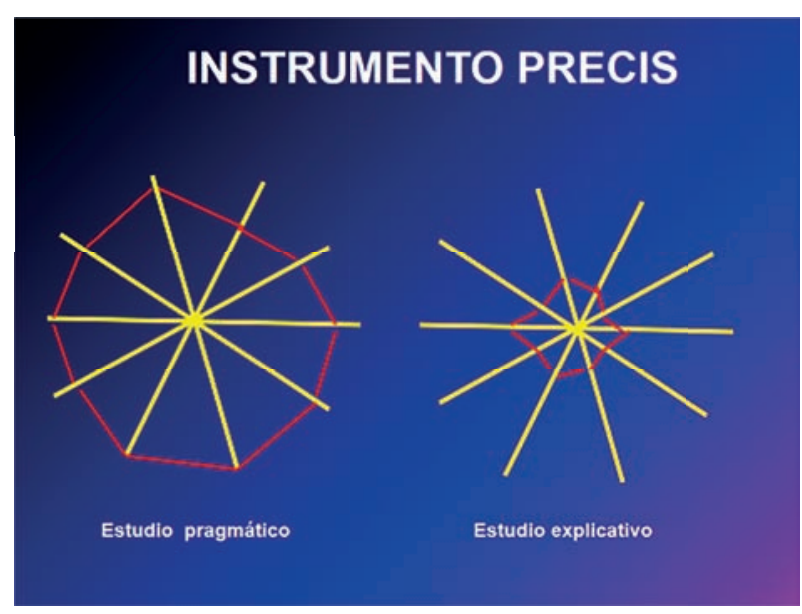

Figura 3. Instrumento PRECIS (pragmatic-explanatory continuum indicator summary). La descripción de la figura está en el texto.

iniciaron el tratamiento, se sobreestima el beneficio de la misma). Hay en estos estudios confusión por indicación (se indica determinada conducta o tratamiento en los que pueden recibirla, y por tanto tienen condiciones que se vinculan con la evolución más allá de lo que se haga) y confusión residual (factores que influyen en el pronóstico y que no son tomados en cuenta en el análisis de las características basales). Por supuesto que también en el caso de los estudios observacionales hay limitaciones económicas: mantener un registro aceitado no es sencillo y demanda de grandes esfuerzos. La vinculación de diferentes bases administrativas es una forma de implementar un seguimiento adecuado de los pacientes, pero por su naturaleza puede haber errores en la forma de caratular diversos eventos?

Los estudios observacionales tienen desde ya una serie de ventajas: alta validez externa, definen la adherencia real a una estrategia o intervención, mayor duración de seguimiento y detección de eventos y efectos adversos poco frecuentes².

En los últimos años se ha revalorizado el papel de los registros de pacientes. Se define a un registro como un sistema organizado que usa métodos de estudio observacional para recolectar datos uniformes (clínicos y otros) para evaluar resultados específicos para una población definida por una enfermedad, afección o exposición particulares y que sirve a uno o más propósitos predeterminados científicos, clínicos o de política sanitaria. La recolección de los datos se hace con un propósito definido, con definiciones preespecificadas de datos coleccionados de manera uniforme, que reflejan el estado clínico del paciente. Al menos un elemento del registro es activo. Pueden enriquecerse al vincularse con otras bases de datos; en general son estudios de cohorte, pero pueden albergar otro tipo de estudios. Son esenciales para describir la historia natural de la enfermedad, definir efectividad y costo efectividad en el mundo real, definir seguridad y daño potencial de las intervenciones, describir calidad de cuidado y barreras en la atención y son fuente de datos para la investigación de resultados centrados en el paciente y, dato no menor, base para la realización de estudios de intervención $n^{8,9}$. Si se va a considerar solo la información proveniente de estudios observacionales, algunas aproximaciones estadísticas pueden intentar salvar las limitaciones mencionadas: análisis multivariado, puntaje de propensión ${ }^{10}$, diseños de nuevo usuario, modelos estructurales marginales, variables instrumentales ${ }^{11}$.
Supongamos que en una población de pacientes con fibrilación auricular se compara la evolución de los pacientes tratados o no con anticoagulantes. Vemos que los anticoagulados tienen mejor evolución. El tratamiento ha sido decidido en cada caso por el médico de cabecera, no se trata de una intervención aleatoria. Como es claro que debe haber sesgos y confundidores vinculados con la diferente evolución de uno y otro grupo, una primera forma de resolver el problema es llevar a cabo un análisis multivariado, en que la variable predicha o resultado sea, por ejemplo, mortalidad; y entre las variables predictoras consideremos todas aquellas que sabemos vinculadas con la evolución, pero también el tratamiento anticoagulante. Si ajustando por todas las variables citadas, el tratamiento anticoagulante aparece como predictor independiente de mortalidad, podemos suponer que esa relación realmente existe. Pero es claro que hemos ajustado solo por las variables conocidas, y que debe haber otras variables confundidoras desconocidas (por las que no pudimos ajustar) que pueden ser las verdaderas responsables de lo observado.

Otro de los procedimientos más empleados es la confección de un puntaje de propensión. ¿En qué consiste? En el estudio de observación que citamos se empieza buscando (mediante regresión logística) cuáles son los predictores independientes de uso de anticoagulantes en la población considerada. Con esos predictores se construye un score o puntaje de propensión, que asume para cada individuo observado un valor, de acuerdo a la presencia o ausencia de cada predictor. Se supone que cuanto mayor el puntaje, mayor la probabilidad de estar tratado con anticoagulantes. Pero no todos los que tienen un puntaje alto están efectivamente tratados con esas drogas, ni todos los que tienen puntaje bajo no lo están. Lo que se hace es, para cada valor del puntaje de propensión (que implica determinada probabilidad de estar tratado), aparear una persona efectivamente tratada y una que no lo está. Se generan entonces dos cohortes, que tienen igual puntaje de propensión promedio para recibir tratamiento, pero una de las cohortes está efectivamente tratada y la otra no. Como se comprenderá, de esta manera se busca simular lo que pasa en un estudio aleatorizado, en que personas con igual probabilidad de ser tratadas o no, reciben la intervención en forma aleatoria. Pero el procedimiento tiene su talón de Aquiles: cuando aleatorizamos repartimos por igual las características conocidas y las desconocidas en ambas ramas; cuando construimos un puntaje de propensión, solo repartimos por igual las características conocidas, y por lo tanto no podemos excluir que haya "algo" desconocido, verdaderamente responsable de la diferente evolución de ambos grupos. Los defensores del empleo de análisis multivariado o uso de un puntaje de propensión para equiparar estudios observacionales a aleatorizados sostienen que si la mayoría de las variables que se sabe relacionadas con la evolución aparecen en el análisis, la variable desconocida tendría que tener muy alta fuerza de asociación con el evento para cambiar el sentido de la observación ${ }^{10}$.

Indudablemente, el surgimiento del concepto de big data, con la posibilidad de manejar y analizar simultáneamente datos poblacionales de gran cantidad de bases de distinta índole-administrativas, de estudios y análisis, prescriptivas, de hospitalización, etc.- implicará un avance notable, y que por ahora solo vislumbramos, en nuestra ca- 
pacidad de emplear datos observacionales y deducir de ellos reglas predictivas que permitan acercarnos a una medicina de precisión ${ }^{12}$. ¿Qué hacer frente a esta contradicción entre estudios observacionales y aleatorizados, y cómo potenciar las ventajas de cada uno? La realización de estudios aleatorizados pragmáticos, que conserven la aleatorización pero con un diseño simple y con criterios de inclusión y exclusión más laxos, y que reproduzcan las condiciones normales de seguimiento de los pacientes con la frecuencia habitual de visitas y prácticas es una opción ${ }^{5}$. Otra, la realización de estudios aleatorizados basados en registros, donde en principio todos los incluidos pueden ser sometidos al estudio con amplia validez externa ${ }^{13}$.

De acuerdo con las palabras de Sir Michael Rawlins, cabeza de NICE (the National Institute for Health and Care Excellence): "Experimento, observación y matemáticas individual y colectivamente tienen un rol crucial en proveer la base de evidencia para la moderna terapéutica. Argumentos sobre la importancia relativa de cada uno son una distracción innecesaria. Las jerarquías de evidencia deberían ser reemplazadas por aceptar e incluso abrazar una diversidad de aproximaciones"14. Observación y experimentación deben ser vistas como aliadas, y no como enemigas. Cada una potencia las ventajas de la otra, y disimula al menos en parte sus flaquezas ${ }^{15}$.

\section{OBSERVACIÓN VS. EXPERIMENTACIÓN EN LA PRÁCTICA COTIDIANA Y EL VERDADERO PROBLEMA}

Suele plantearse la oposición de argumentos acerca de tomar conductas cuando no hay estudios aleatorizados acerca su implementación, o acerca de qué hacer con los potenciales hallazgos.

Vayamos por partes. La inmensa mayoría de las conductas que tomamos a diario en la práctica médica no se basan en estudios aleatorizados. Cuando nos remitimos a las guías de práctica sucede algo similar. De 133 recomendaciones que formula la guía de práctica de Insuficiencia Cardíaca de la Sociedad Europea de Cardiología de 2016 para la insuficiencia cardíaca crónica ${ }^{16}$, solo el $24,8 \%$ son A; el 30,8\% son B y el $44,4 \%$ son C. Y esto es particularmente notable cuando nos referimos a conductas diagnósticas. Si nos remitimos a este apartado en el Consenso de Insuficiencia Cardíaca de la Sociedad Argentina de 2016, de 19 recomendaciones, 13 son C y 6 son B. No hay recomendaciones sobre diagnóstico con evidencia A. Es decir, que en general las conductas en este campo son en gran parte empíricas; no se sustentan ni siquiera en estudios observacionales. Pareciera no haber verdadero interés en encontrar justificación firme para la mayor parte de nuestras prácticas ${ }^{17}$.

Muchas veces el examen cuidadoso de los datos de que se dispone puede darnos sorpresas. Por ejemplo, la realización de un electrocardiograma (ECG) basal, o incluso uno de esfuerzo, es parte habitual de la práctica cardiológica. Muchos médicos consideran que la informa- ción que brinda complementa la que entregan las variables clínicas. Una revisión sistemática y categorización de la evidencia disponible formulada a pedido de la Fuerza de Tareas de Servicios Preventivos de los Estados Unidos (USPSTF, por su sigla en inglés) acaba de publicarse. Y, para gran sorpresa de todos, resulta que no hay estudios que hayan explorado la utilidad del ECG de esfuerzo en personas de bajo riesgo; $y$ en aquellos de riesgo moderado a alto no hay evidencia firme de repercusión favorable, y la mejoría de los modelos pronósticos tradicionales con el agregado de datos ECG es al menos discutible ${ }^{18}$. De allí que en estos pacientes se mantenga que no hay información consistente. Es tremendamente ilustrativo que con la cantidad incontable de ECG que se llevan a cabo en millones y millones de personas cada año, preguntas sencillas sobre su utilidad no puedan ser respondidas. Este es solo un ejemplo de cómo conductas que entendemos "indiscutibles" pueden ser sometidas a escrutinio, y no encontrar evidencia clara para implementarlas.

Podemos sí sostener que parece excesivo entender como "evidencia" solo lo que proviene de ensayos aleatorizados. En realidad, la Medicina Basada en la Evidencia fue, en sus orígenes, la idea de basar nuestra conducta en la mejor evidencia disponible ${ }^{19}$. Esa información puede provenir entonces de estudios aleatorizados u observacionales. La asignación aleatoria, en virtud de todo lo expuesto, no es garantía indiscutible de evidencia fuerte. Hay estudios aleatorizados de mejor o peor calidad, más o menos creíbles, con conclusiones más o menos extrapolables a todos nuestros pacientes. En el otro extremo, hay estudios observacionales de mejor o peor calidad, y sobre los que podemos formular las mismas apreciaciones. Así como la aleatorización por sí sola no es garantía indiscutible, tampoco lo es la observación, si no es rigurosa, completa y sólidamente constituida. Cada una de las fuentes de información debe ser adecuadamente evaluada, analizada y juzgada. Y seguiremos discutiendo hasta el hartazgo cada aseveración; muchas verdades inconmovibles que se sostuvieron durante décadas se han derrumbado ante la aparición de nuevos hallazgos.

\section{CONCLUSIONES}

Está claro que a lo que no se debe renunciar de ninguna manera es al imperativo de tener siempre en cuenta la mejor información, la más pura y menos sesgada. En países como los nuestros, creo que en muchos aspectos estamos en una etapa previa de la discusión. No se trata del antagonismo entre datos de estudios observacionales o aleatorizados; se trata de tener datos. Es la falta de un registro elemental de lo que se hace (en forma integral, no aqueIlo que surge de iniciativas parciales y bienintencionadas) el pecado original de nuestra práctica. A remediar el mismo deberían dirigirse nuestros esfuerzos. Si la necesidad de disponer de información se hace carne en nuestras sociedades, es de esperar que lo que sigue se dará por añadidura. 


\section{BIBLIOGRAFÍA}

1. Diamond GA. Randomized trials, observational registries, and the foundations of evidence-based medicine. Am J Cardiol 2014;113(8):1436-41.

2. Hannan EL. Randomized clinical trials and observational studies: guidelines for assessing respective strengths and limitations. JACC Cardiovasc Interv 2008;1(3):211-7.

3. Faraoni D, Schaefer ST. Randomized controlled trials vs. observational studies: why not just live together? BMC Anesthesiol 2016;16(1):102.

4. Thorpe KE, Zwarenstein M, Oxman AD, Treweek S, Furberg CD, Altman DG et al. A pragmatic-explanatory continuum indicator summary (PRECIS): a tool to help trial designers. J Clin Epidemiol 2009;62(5):464-75.

5. Ford I, Norrie J. Pragmatic Trials. N Engl J Med 2016:375(5):454-63.

6. Balshem H, Helfand M, Schunemann HJ, Oxman AD, Kunz R, Brozek J et al. GRADE guidelines: 3. Rating the quality of evidence. J Clin Epidemiol 2011;64(4):401-6.

7. leva $F$, Gale $C P$, Sharples $L D$. Contemporary roles of registries in clinical cardiology: when do we need randomized trials? Expert Rev Cardiovasc Ther 2014;12(12):1383-6.

8. Hemkens $L G$, Contopoulos-loannidis DG, loannidis JP. Agreement of treatment effects for mortality from routinely collected data and subsequent randomized trials: meta-epidemiological survey. BMJ 2016;352:1493

9. Gliklich RE, Dreyer NA, Leavy MB. Registries for Evaluating Patient Outcomes: A User's Guide (2014,Tercera edición). Estados Unidos: Rockville (MD); Agency for Healthcare Research and Quality (US).

10. Kitsios GD, Dahabreh IJ, Callahan S, Paulus JK Campagna AC Dargin IM. Can We Trust Observational Studies Using Propensity Scores in the Critical Care Literature? A Systematic Comparison With Randomized Clinical Trials. Crit Care Med 2015:43(9):1870-9.
11. Hlatky MA, Winkelmayer WC, Setoguchi S. Epidemiologic and statistical methods for comparative effectiveness research. Heart Fail Clin 2013;9(1):29-36.

12. Van Poucke S, Thomeer M, Hadzic A. 2015, big data in healthcare: for whom the bell tolls? Crit Care 2015;19:171.

13. Jones WS, Roe MT, Antman EM, Pletcher MJ, Harrington RA, Rothman RL et al. The Changing Landscape of Randomized Clinical Trials in Cardiovascular Disease. $J$ Am Coll Cardiol 2016;68(7):1898-1907.

14. Rawlins M. De testimonio: on the evidence for decisions about the use of therapeutic interventions. Lancet 2008;372(9656):2152-61.

15. Booth CM, Tannock IF. Randomised controlled trials and population-based observational research: partners in the evolution of medical evidence. $\mathrm{Br} J \mathrm{Cancer}$ 2014;110(3):551-5.

16. Ponikowski P, Voors AA, Anker SD, Bueno H, Cleland JGF, Coats AJS et al. 2016 ESC Guidelines for the diagnosis and treatment of acute and chronic heart failure: The Task Force for the diagnosis and treatment of acute and chronic heart failure of the European Society of Cardiology (ESC)Developed with the special contribution of the Heart Failure Association (HFA) of the ESC. Eur Heart J 2016:37(27):21292200.

17. Sociedad Argentina de Cardiología. Consenso de Insuficiencia Cardíaca Crónica. Rev Arg Cardiol 2016:84(3):1-50.

18. Jonas DE, Reddy S, Middleton JC, Barclay C, Green J, Baker C et al. Screening for Cardiovascular Disease Risk With Resting or Exercise Electrocardiography: Evidence Report and Systematic Review for the US Preventive Services Task Force. JAMA 2018;319(22):2315-2328

19. Sackett DL, Rosenberg WM, Gray JA, Haynes RB, Richardson WS. Evidence based medicine: what it is and what it isn't. BMJ 1996;312(7023):71-2. 


\section{Magnefen}

El fenofibrato más ventajoso en dislipemia aterogénica

- El fenofibrato de colina se disocia a su forma activa, ácido fenofíbrico, en el tubo digestivo

- El ácido fenofíbrico no requiere metabolismo de primer paso hepático para su activación

- El ácido fenofíbrico tiene mayor biodisponibilidad plasmática que el fenofibrato

- Tratamiento de elección en dislipemia mixta e hipertrigliceridemia severa

- Efectivo co-administrado con estatinas

- Óptima tolerabilidad en monoterapia como en combinación

- Puede administrarse con o sin alimentos

\section{INDICACIONES}
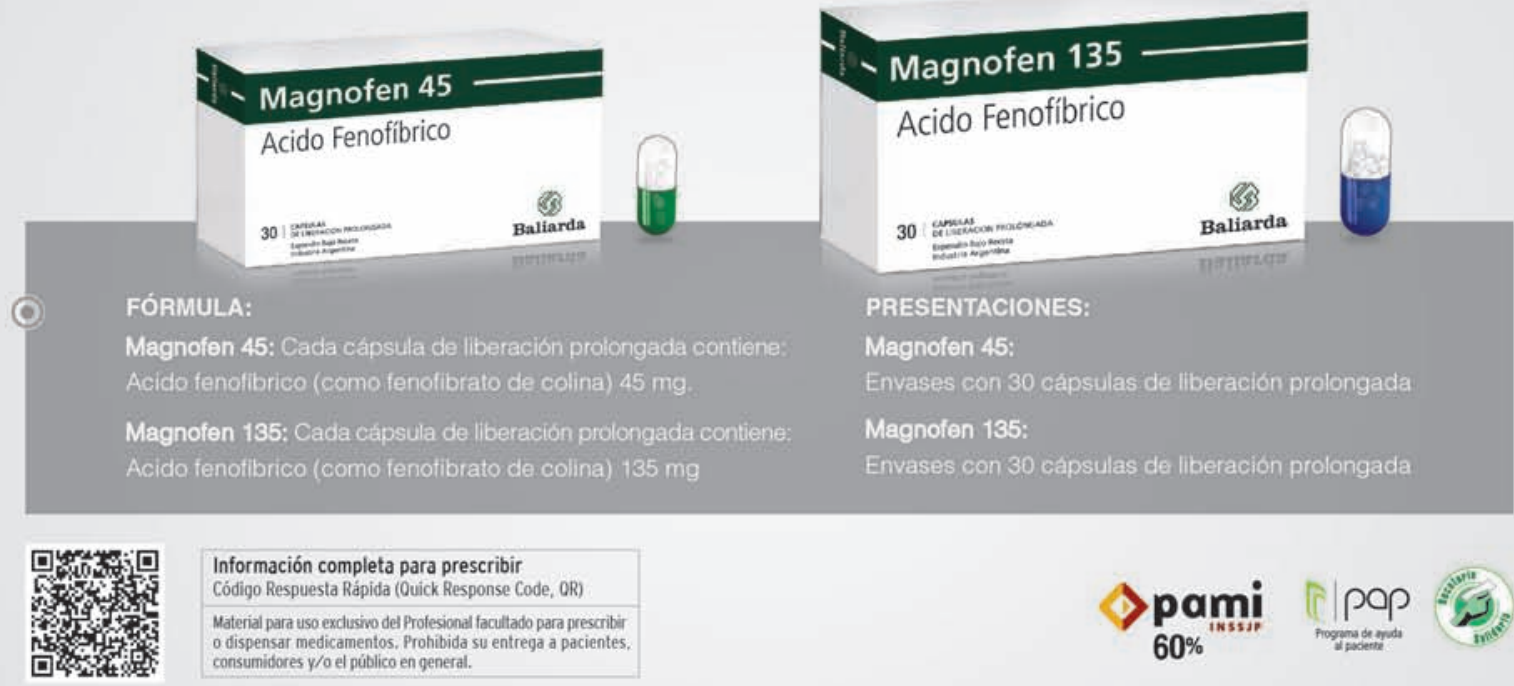
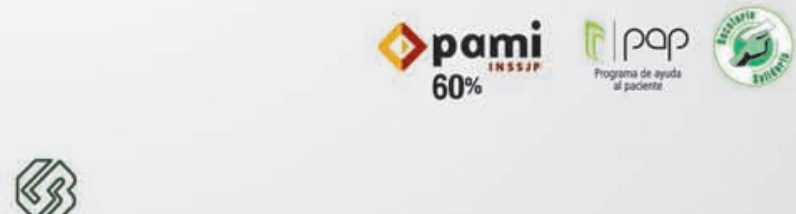

Baliarda

Vida con salud

www.baliarda.com.ar 


\title{
COMPLICACIONES DE LA VENTILACIÓN MECÁNICA EN EL POSOPERATORIO DE CIRUGÍA CARDIOVASCULAR
}

\section{COMPLICATIONS OF MECHANICAL VENTILATION IN POSTOPERATIVE CARE OF CARDIOVASCULAR SURGERY}

\author{
FERNANDO RÍOS', NICOLÁS DIEZ²
}

\section{RESUMEN}

La cirugía cardíaca conlleva una variedad de complicaciones pulmonares, algunas de las cuales contribuyen a la lesión pulmonar o síndrome de dificultad respiratoria aguda, y se asocia con aumento de la permeabilidad vascular pulmonar, el incremento del agua pulmonar extravascular y/o la generación de atelectasias. Los mecanismos implicados en el desarrollo de estas incluyen dos causas fundamentales: el trauma del procedimiento quirúrgico y el efecto de la ventilación mecánica sobre un entorno inflamatorio. Esta revisión se enfocará en abordar las diferentes efectos cardiovaculares y complicaciones de la ventilación mecánica en pacientes en el posoperatorio de cirugía cardíaca, y además se revisarán diferentes estrategias terapéuticas para minimizar la lesión pulmonar.

Palabras clave: respiración artificial; intubación; cirugía torácica.

\section{ABSTRACT}

Cardiac surgery involves a variety of pulmonary complications, some of which contribute to lung injury or acute respiratory distress syndrome, and is associated with increased pulmonary vascular permeability, increased extravascular pulmonary water, and / or the generation of atelectasis. The mechanisms involved in the development of these include two fundamental causes: the trauma of the surgical procedure and the effect of mechanical ventilation on an inflammatory environment. This review will focus on addressing the different cardiovascular effects and complications of mechanical ventilation in patients in the postoperative period of cardiac surgery, and will also review different therapeutic strategies to minimize lung injury.

Keywords: respiration; intubation; thoracic surgery.

REVISTA CONAREC 2018;33(146):221-225 | DOI:10.32407/RCON/2018146/0221-0225

\section{INTRODUCCIÓN}

La ventilación mecánica (VM) también se denomina ventilación con presión positiva. Consiste en la entrega de una mezcla predeterminada de gases (oxígeno y otros gases) de manera forzada en las vías respiratorias centrales que luego fluye hacia los alvéolos. A medida que estos gases ingresan a los pulmones durante la inspiración, la presión intraalveolar aumenta. La espiración se produce pasivamente liberando el gas entregado por diferencia de presión entre la vía aérea y la atmósfera. Los principales beneficios de la VM son la mejora del intercambio de gases y la disminución del trabajo respiratorio; mejora el intercambio de gases al mejorar la relación ventilación-perfusión (V/Q). El trabajo respiratorio puede aumentar debido a la alteración de la mecánica pulmonar (p. ej., aumento de la resistencia de las vías respiratorias o disminución de la compliance) o por aumento de la demanda respiratoria (p. ej., acidosis, dolor, etc.). El esfuerzo requerido para mantener este trabajo respiratorio elevado puede ocasionar fatiga de los músculos respiratorios e insuficiencia respiratoria. La VM

1. Jefe de Terapia Intensiva en Sanatorio Las Lomas. Staff de Terapia Intensiva de Adultos, Hospital Nacional Alejandro Posadas.

2. Residente de Terapia Intensiva de Adultos, Hospital Nacional Alejandro Posadas.

$\triangle$ Correspondencia:Dr. Fernando Ríos. conarecrevista@gmail.com

Los autores declaran no tener conflictos de intereses.

Recibido: 06/06/2018| Aceptado: 01/08/2018 puede asumir parte o todo el trabajo respiratorio, permitiendo que los músculos ventilatorios se recuperen.

La cirugía cardíaca conlleva una variedad de complicaciones pulmonares, algunas de las cuales contribuyen a la lesión pulmonar o síndrome de dificultad respiratoria aguda (SDRA). La cirugía cardíaca se asocia con aumento de la permeabilidad vascular pulmonar, el incremento del agua pulmonar extravascular (EVLW) y/o la generación de atelectasias. Estas alteraciones pueden actuar solas o en conjunto, y pueden contribuir a las anormalidades mecánicas o del intercambio gaseoso, como el desarrollo de hipoxemia y dependencia temporal del ventilador. También pueden estar relacionadas a procesos inflamatorios de origen múltiple, como la circulación extracorpórea, el daño por isquemia-reperfusión, la presencia de derrame pleural y la ventilación mecánica per se o daño inducido por la ventilación mecánica.

\section{VENTILACIÓN MECÁNICA Y CIRUGÍA CARDÍACA}

En un adulto sano normal en posición vertical, la capacidad residual funcional (CRF) es aproximadamente de 3 litros y disminuye en 0,70,8 litros en la posición supina cuando el contenido abdominal empuja el diafragma en dirección cefálica. Esto se acentúa aún más con la anestesia y la parálisis, ya que la presión abdominal se transmite fácilmente a la cavidad torácica. Luego de la cirugía cardiovascular, la CRF se reduce un 40\% en contraste al 20\% observado en el resto de los posoperatorios ${ }^{1,2}$. Si bien no está clara la razón para esta diferencia, varios factores parecen contribuir y confluir en un evento inflamatorio pulmonar. La circulación extracorpórea (CEC), la injuria induci- 
Tabla 1. Efecto cardiovascular de la ventilación mecánica. PVC: presión venosa central. VD: ventrículo derecho. VI: ventrículo izquierdo.

\section{Cambios generados por la ventilación mecánica}

Incremento en la presión intratorácica.

Disminución del volumen sanguíneo pulmonar y aumento del volumen sanguíneo sistémico.

Disminución del retorno venoso.

Disminución del volumen sistólico del VD.

Disminución en la presión arterial pulmonar.

Disminución en las presiones de llenado de los ventrículos.

Disminución del volumen sistólico del VI.

\section{Comentario}

La presión positiva aplicada a los pulmones provoca la compresión del parénquima pulmonar contra la pared del tórax

Durante la fase inspiratoria de la ventilación de presión positiva, una fracción del volumen sanquíneo pulmonar se desplaza a la circulación sistémica. Esto no aumenta la PVC, porque la circulación venosa sistémica puede absorber fácilmente este volumen extra.

La mayor presión intratorácica impide el retorno venoso sistémico de sangre al VD.

La disminución del retorno venoso al VD conduce a la disminución de su volumen sistólico.

Disminución del volumen sistólico del VD reduce el volumen sanguíneo (presión) en las arterias pulmonares.

La disminución del volumen sistólico del VD y la caída de la presión de la arteria pulmonar y provoca una reducción de la precarga y del volumen sistólico del VI. da por la isquemia-reperfusión y la VM juegan un rol importante en el proceso inflamatorio pulmonar posquirúrgico. Se han observado reducciones marcadas en el FEV 1 y la capacidad vital forzada (CVF) hasta 5 días después de la cirugía, y estos cambios pueden persistir semanas después de la operación ${ }^{3}$.

Atelectasia deriva de las palabras griegas ateles y ektasis, que significa expansión incompleta. La atelectasia es un colapso pulmonar con pérdida del volumen pulmonar, ya sea una parte o la totalidad de un pulmón. Pueden deberse a mecanismos de obstrucción/compresión de la vía aérea o por reabsorción al administrar fracciones inspiradas de oxigeno elevadas y a la pérdida del surfactante que favorece los fenómenos de apertura y cierre alveolar cíclico ${ }^{4,5}$. En algunos pacientes la presencia de atelectasias podría ser una de las principales causas de daño pulmonar posquirúrgi$\mathrm{CO}^{6,7}$. Ante la presencia de una atelectasia son varias las situaciones que inducen daño, en primer lugar, los alvéolos que rodean la atelectasia se ven sometidos a fuerzas de tracción de alta energía como consecuencia de la distribución desigual de las fuerzas; dicha energía es transmitida al epitelio capilar generando aumento de la permeabilidad, edema y liberación de proteínas que inactivan más surfactante. El principal determinante para este evento es el volumen corriente, que genera incrementos de la presión transpulmonar en las unidades alveolares vecinas a las zonas de atelectasia. Otra explicación es la desigual entrega de energía en el sistema pulmonar, por la distribución del volumen de manera no uniforme, que tiene como consecuencia áreas de sobredistensión asociado a áreas de colapso y reapertura que perpetúan el ciclo de daño $0^{8,9}$.

Podemos resumir al decir que la VM induce al proceso inflamatorio desde dos aspectos, uno mecánico y otro biológico. Por un lado, se somete al alvéolo a volúmenes y presiones que pueden ser injuriantes comprometiendo la integridad del epitelio alveolar con el consecuente edema y ocupación del espacio alveolar ${ }^{10,11}$; por otro lado, el daño biológico hace referencia a la liberación de citoquinas proinflamatorias y cambios en la funcionalidad del endotelio capilar que resulta en la perpetuación del proceso inflamatorio, que una vez instalado repercute a nivel sistémico constituyendo lo que se denomina biotrauma ${ }^{12}$

No son claras las vías por las cuales los eventos mecánicos producen la liberación de citoquinas. Tanto el cierre y reapertura cíclica como las presiones transpulmonares aumentadas generan cambios en el citoesqueleto que iniciarían cascadas de señalización intracelular y consecuentemente liberación de factores proinflamatorios ${ }^{13}$. Una vez iniciado el proceso pulmonar, la actividad proinflamatoria puede repercutir a nivel sistémico, esto se evidencia por el incremento sérico de citoquinas como el factor de necrosis tumoral alfa (TNF-alfa), la interleucina 6 (IL-6) y la interleucina 8 (IL-8) 12,14,15

La mayor prevalencia de disfunciones pulmonares en el posoperatorio de cirugía cardiovascular respecto de otras cirugías parece responder a varias causas, entre ellas el estrés mecánico y el biotrauma consecuente. En este contexto, las estrategias ventilatorias durante el procedimiento y luego del mismo son determinantes de la evolución de los pacientes.

\section{EFECTO CARDIOVASCULAR DE LA VENTILACIÓN MECÁNICA}

El principal cambio producido por la ventilación mecánica es el cambio a presión positiva intratorácica. La magnitud del impacto depende de varios factores como la función miocárdica prequirúrgica, el estado del volumen intravascular, el nivel de presión positiva intratorácica o las patologías valvulares (Tabla 1). La VM produce cambios hemodinámicos, afectando fundamentalmente la precarga y la poscarga ventricular.

La presión positiva intratorácica reduce el retorno venoso y disminuye la diferencia de presión entre el sistema venoso sistémico y la aurícula derecha, consecuentemente reduce la precarga del ventrículo derecho (VD), esto disminuye el volumen de fin de diástole y del volumen minuto cardíaco.

La reducción de la CRF y las atelectasias presentes en el paciente sometido a cirugía cardiovascular generan una disminución del volumen pulmonar al final de espiración y vasoconstricción hipóxica generando un aumento de la resistencia vascular pulmonar, esto ocasiona el aumento de la poscarga del VD. Para disminuir el impacto de estos cambios, la aplicación de presión positiva de fin de espiración (PEEP) es fundamental como estrategia de ventilación mecánica. E objetivo es evitar el colapso alveolar y mejorar la oxigenación que resultará en la optimización del gasto cardíaco ${ }^{16,19}$

El ventrículo izquierdo (VI) sufre una disminución del volumen de fin de diástole por la caída del gasto cardíaco derecho, pero la poscarga del VI se reduce por acción de la PEEP, ya que disminuye su presión transmural mejorando el gasto cardíaco izquierdo. 
Tabla 2. Modificaciones que produce el uso de PEEP ideal (óptima) y valores de PEEP en exceso. PEEP: presión positiva en fin de espiración. VI: ventrículo izquierdo.

\begin{tabular}{lll} 
& PEEP óptima & PEEP en exceso \\
\hline Presión intratorácica & Incrementa & Incrementa \\
\hline Capacidad residual funcional & Incrementa & Incrementa \\
\hline Compliance pulmonar & Incrementa & Disminuye \\
\hline $\mathrm{PaCO}_{2}$ & Disminuye & Incrementa \\
\hline Shunt & Disminuye & Incrementa \\
\hline Espacio muerto & Disminuye & Incrementa \\
\hline Gasto cardíaco & Incrementa & Disminuye \\
\hline Resistencia vascular pulmonar & Normal & Incrementa \\
\hline Poscarga del VI & Disminuye & Disminuye
\end{tabular}

Durante la inspiración, el aumento del volumen pulmonar comprime la sangre fuera del lecho pulmonar, aumentando la precarga del VI. La combinación de aumento de la precarga y la disminución de la poscarga del VI durante la inspiración resulta en un incremento del volumen sistólico del VI y la presión arterial sistólica durante la ventilación mecánica ${ }^{20}$.

En contraposición a esto la VM produce significativa hipotensión en aquellos pacientes con disminución del volumen intravascular (hipovolemia), la presión positiva intratorácica tendrá mayor impacto en la precarga del VD y consecuentemente en la caída del volumen de fin de diástole del $\mathrm{V}^{21-24}$. Por ello, se debe asegurar un volumen intravascular adecuado y además como ya se ha mencionado, utilizar una estrategia ventilatoria que evite el colapso alveolar y la vasoconstricción hipóxica. Un óptimo gasto cardíaco derecho, que garantice un adecuado volumen de fin de diástole del VI, con la concomitante disminución de la poscarga del VI generada por la presencia de presión positiva intratorácica son los objetivos fundamentales en el paciente sometido a cirugía cardiovascular.

Durante el destete de la VM, cuando el paciente comienza con esfuerzos inspiratorios, la presión intratorácica negativa produce un aumento en la poscarga del Vl, y el aumento previo en el volumen de eyección del VI se pierde.

Normalmente, el gasto cardíaco aumenta en respuesta a un aumento en la demanda metabólica. Si esta respuesta está limitada, un aumento en el consumo metabólico de oxígeno puede causar una reducción en la saturación de oxígeno venoso mixto y favorecer la hipoxemia, y en algunos pacientes, puede provocar isquemia miocárdica y causar fallo del destete ${ }^{25}$.

\section{CIRCULACIÓN EXTRACORPÓREA}

La CEC es un sistema artificial de soporte hemodinámico y ventilatorio temporal durante la parada cardíaca, necesaria para realizar muchas intervenciones. Está constituida por un oxigenador, una bomba y un sistema de catéteres. El contacto con el material extraño y el sistema de bombeo produce con gran frecuencia un estado de inflamación generalizada. Se ha establecido la relación entre la CEC y la vasoplejía posterior, sin embargo, existen resultados controversiales ${ }^{26}$. Ascione et al. demostraron una menor producción de citoquinas proinflamatorias en pacientes sometidos a cirugía cardiovascular sin CEC respecto de las cirugías con $\mathrm{CEC}^{27}$. Se ha documentado también una menor incidencia de complicaciones pulmonares, extubación precoz y menor tiempo de VM ${ }^{28}$. Se puede concluir que el uso de CEC se asocia a la generación de inflamación y potencia los efectos de la VM a presión positiva.

\section{TERAPÉUTICAS PARA MINIMIZAR LA LESIÓN PULMONAR: ESTRATEGIAS DE PULMÓN ABIERTO (OPEN LUNG).}

Esta modalidad ventilatoria utilizada en los pacientes con SDRA tiene como objetivo disminuir la cantidad de energía entregada al sistema utilizando bajos volúmenes corrientes con la asociación de valores de PEEP suficientes para evitar la apertura y cierre cíclico en áreas alveolares inestables. Para un uso óptimo requiere la implementación concomitantemente de maniobras de reclutamiento, esta aproximación se considera actualmente como una de las mejoras estrategias ventilatorias para el SDRA ${ }^{29,30}$. Los objetivos son alcanzar parámetros gasométricos apropiados $\left(\mathrm{pH}>7,20 ; \mathrm{PaO}_{2}>65 \mathrm{mmHg}\right.$ ) en contexto de una PEEP que logre contrarrestar el colapso alveolar por la disminución de la compliance pulmonar. Es fundamental mantener presiones transpulmonares que no sean lesivas, esto se consigue evitando la presencia de una gran amplitud entre la presión meseta y la PEEP (driving pressure $<16 \mathrm{cmH}_{2} \mathrm{O}$ ), además de evitar generar sobredistensión alveolar (valores de presión meseta $<28 \mathrm{cmH}_{2} \mathrm{O}$ ) ${ }^{31}$. Para lograr estos objetivos es necesario la utilización de volúmenes corrientes de 6 ml kg/peso teórico o menores si la presión meseta es elevada. La realización de maniobras de reclutamiento busca la apertura de unidades colapsadas y permite la titulación de un valor de PEEP que evite el colapso espiratorio con mínimo efecto hemodinámico ${ }^{32,33}$. El uso sistemático de maniobras de reclutamiento ha caído en desuso en los últimos tiempos por falta de evidencia en la relación beneficios y daño inducidos por las maniobras de reclutamiento ${ }^{34,35}$. Las maniobras de reclutamiento consisten en la aplicación transitoria (minutos) de altas presiones inspiratorias y representan un riesgo potencial de barotrauma, o trastornos hemodinámicos deletéreos ${ }^{33,36,37}$. Sin embargo, su utilización en el contexto de las cirugías cardíacas se sustenta principalmente para revertir las atelectasias. Amato et al. desarrollaron un protocolo con maniobras de reclutamiento en pacientes sometidos a cirugía cardiovascular. Este estudio prospectivo con asignación aleatoria evaluó la eficacia de una maniobra de reclutamiento intensiva vs un reclutamiento moderado, con resultados favorables para las maniobras de reclutamiento intensivas respecto a complicaciones pulmonares postoperatorias, días de ventilación mecánica y el tiempo de hospitalización ${ }^{38}$.

En la práctica diaria establecer el valor exacto de PEEP, no está aclarado. Se considera PEEP óptima al valor que genera un menor efecto hemodinámico y permite la mayor cantidad de unidades alveolares estables (mejor compliance, sin colapso espiratorio) (Figura 1). Dichos valores de PEEP pueden establecerse mediante el uso de balón esofágico, por valoración de la compliance o usando tablas de $\mathrm{PEEP} / \mathrm{FiO}_{2}$. Recientemente se ha propuesto el uso de tomografía de impedancia como estrategia para titular la PEEP. Cada clínico deberá evaluar la situación particular e incluso los cambios que en el paciente suceden con la modificación de los volúmenes pulmonares al titular PEEP (Tabla 2). 


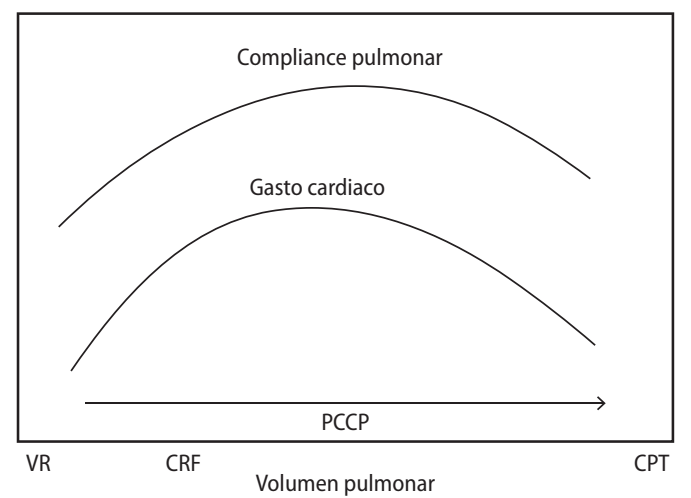

Figura 1. Relación entre PEEP, compliance pulmonar y gasto cardíaco. El incremento en los valores de PEEP aumenta los volúmenes pulmonares, al generar sobredistensión alveolar reducen el gasto cardiaco. El valor de PEEP ideal debería producir la mejor relación entre gasto cardiaco y compliance pulmonar. VR: volumen residual. CRF: capacidad residual pulmonar. CPT: capacidad pulmonar total. PEEP. presión positiva en fin de espiración.

\section{VENTILACIÓN DURANTE CEC}

La hipoventilación durante la CEC genera una mayor incidencia de atelectasias, edema alveolar, disminución de la compliance pulmonar y mayor tasa de infecciones. Existen diversas propuestas para disminuir estas complicaciones ${ }^{39}$. Mantener una presión constante en la vía aérea durante la CEC con presión positiva continua en la vía aérea de $10 \mathrm{~cm} \mathrm{H}_{2} \mathrm{O}$ ha demostrado mejorar el perfil gasométrico en el poso- peratorio ${ }^{40}$. Jhon et al. demostraron que mantener una ventilación a $5 \mathrm{ml} / \mathrm{kg}$ de peso teórico durante la CEC se asocia con una mejor gasometría, un mejor perfil en la mecánica pulmonar y logra menores tiempos de ventilación mecánica y disminución de las complicaciones pulmonares postoperatorias ${ }^{41}$.

\section{CONCLUSIÓN}

La incidencia de trastornos pulmonares y necesidad de ventilación mecánica después de la cirugía cardíaca es frecuente. Los mecanismos implicados en el desarrollo de alteraciones de la mecánica y el intercambio gaseoso pulmonar incluyen dos causas fundamentales: el trauma del procedimiento quirúrgico y el efecto de la ventilación mecánica sobre un entorno inflamatorio, que es mayor en presencia de CEC. La inflamación pulmonar se agrava por la aplicación de VM inadecuada.

Actualmente, se recomienda iniciar la VM con una estrategia de apertura pulmonar temprana en el procedimiento (después de la intubación orotraqueal) con volúmenes corrientes bajos (volumen corriente $6 \mathrm{ml} / \mathrm{kg}$ y uso de PEEP en valores de 8-14 $\mathrm{CmH}_{2} \mathrm{O}$ ), con uso de maniobras de reclutamiento. Si aplicamos estas estrategias podemos esperar una mejora considerable en los parámetros de intercambio de gases, con una menor elevación de los mediadores inflamatorios, una mejor CRF posoperatoria y una menor incidencia de readmisión en la unidad de terapia intensiva. Finalmente, debemos recordar que todo esto se puede lograr con mínimos efectos hemodinámicos adversos.

\section{BIBLIOGRAFÍA}

1. Bardell T, Legare JF, Buth KJ, Hirsch GM, Ali IS. ICU readmission after cardiac surgery. Eur J Cardiothorac Surg 2003;23(3):354-9.

2. Nicholson DJ, Kowalski SE, Hamilton GA, Meyers MP, Serrette C, Duke PC. Post operative pulmonary function in coronary artery bypass graft surgery patients undergoing early tracheal extubation: a comparison between shortterm mechanical ventilation and early extubation. J Cardiothorac Vasc Anesth 2002;16(1):27-31.

3. Weissman C. Pulmonary function after cardiac and thoracic surgery. Anesth Analg 1999;88(6):1272-9

4. Rusca M, Proietti S, Schnyder P, Frascarolo P, Hedenstierna G, Spahn DR, et al. Prevention of atelectasis formation during induction of general anesthesia. Anesth Analg 2003;97(6):1835-9.

5. Magnusson L, Spahn DR. New concepts of atelectasis during general anaesthesia. Br J Anaesth 2003:91(1):61-72.

6. Magnusson L, Zemgulis V, Wicky S, Tyden H, Thelin S, Hedenstierna G. Atelectasis is a major cause of hypoxemia and shunt after cardiopulmonary bypass: an experimental study. Anesthesiology 1997:87(5):1153-63.

7. Verheij J, van Lingen A, Raijmakers $P G$, Spijkstra JJ, Girbes $A R$, Jansen EK, et al. Pulmonary abnormalities after cardiac surgery are better explained by atelectasis than by increased permeability oedema. Acta Anaesthesiol Scand 2005;49(9):1302-10

8. Hubmayr RD. Perspective on lung injury and recruitment: a skeptical look at the opening and collapse story. Am J Respir Crit Care Med 2002:165(12):1647-53.

9. Cressoni M, Chiumello D, Chiurazzi C, Brioni M, Algieri I, Gotti M, et al. Lung inhomogeneities, inflation and [18F]2-fluoro-2-deoxy-D-glucose uptake rate in acute respiratory distress syndrome. Eur Respir J 2016; 47(1):233-42.

10. Meade MO, Cook DJ, Guyatt GH, Slutsky AS, Arabi YM, Cooper DJ, et al. Ventilation strategy using low tidal volumes, recruitment maneuvers, and high positive end-expiratory pressure for acute lung injury and acute respiratory distress syndrome: a randomized controlled trial. JAMA 2008;299(6):637-45.

11. Briel M, Meade M, Mercat A, Brower RG, Talmor D, Walter SD, et al. Higher vs lower positive end-expiratory pressure in patients with acute lung injury and acute

respiratory distress syndrome: systematic review and meta-analysis. JAMA 2010:303(9):865-73.

12. Ranieri VM, Suter PM, Tortorella C, De Tullio R, Dayer JM, Brienza A, et al. Effect of mechanical ventilation on inflammatory mediators in patients with acute respiratory distress syndrome: a randomized controlled trial. JAMA 1999;282(1):54-61.

13. Uhlig U, Fehrenbach H, Lachmann RA, Goldmann T, Lachmann B, Vollmer E, et al. Phosphoinositide 3-OH kinase inhibition prevents ventilation-induced lung cell activation. Am J Respir Crit Care Med 2004;169(2):201-8.

14. Fisher AB, Chien S, Barakat Al, Nerem RM. Endothelial cellular response to altered shear stress. Am J Physiol Lung Cell Mol Physiol 2001;281(3):L529-33.

15. Pinhu L, Whitehead T, Evans T, Griffiths M. Ventilator-associated lung injury. Lancet 2003;361(9354):332-40.

16. Pinsky MR. Cardiovascular issues in respiratory care. Chest 2005:128(5 Suppl 2):592S-5975

17. Dawson CA, Grimm DJ, Linehan JH. Lung inflation and longitudinal distribution of pulmonary vascular resistance during hypoxia. J Appl Physiol Respir Environ Exerc Physiol 1979;47(3):532-6.

18. Reis Miranda D, Gommers D, Struijs A, Meeder H, Schepp R, Hop W, et al. The open lung concept: effects on right ventricular afterload after cardiac surgery. British journal of anaesthesia 2004:93(3):327-32.

19. Agro F, Barzoi G, Doyle DJ, Manieri A. Reduction in pulmonary shunt using the Open Lung Concept. Anaesthesia. 2004;59(6):625-6.

20. Boerlage-van Dijk K, Meregalli PG, Planken RN, Koch KT, Baan J, Jr. Percutane ous left ventricular partitioning device for chronic heart failure. Neth Heart $J$ 2012;20(12):513-515.

21. Pinsky MR. The hemodynamic consequences of mechanical ventilation: an evolving story. Intensive Care Med. 1997;23(5):493-503.

22. Luecke T, Pelosi P. Clinical review: Positive end-expiratory pressure and cardiac out put. Crit Care 2005;9(6):607-21,

23. Jardin F, Dubourg O, Margairaz A, Bourdarias JP. Inspiratory impairment in right ventricular performance during acute asthma. Chest 1987;92(5):789-95. 
24. Jardin F, Farcot JC, Boisante L, Curien N, Margairaz A, Bourdarias JP. Influence of positive end-expiratory pressure on left ventricular performance. N Engl Med 1981;304(7):387-92.

25. Jubran A, Mathru M, Dries D, Tobin MJ. Continuous recordings of mixed venous oxygen saturation during weaning from mechanical ventilation and the ramifications thereof. Am J Respir Crit Care Med 1998;158(6):1763-9.

26. Groeneveld $A B$, Jansen EK, Verheij J. Mechanisms of pulmonary dysfunction after on-pump and off-pump cardiac surgery: a prospective cohort study. J Cardiothorac Surg 2007,2:11.

27. Ascione R, Lloyd CT, Underwood MJ, Lotto AA, Pitsis AA, Angelini GD. Inflammatory response after coronary revascularization with or without cardiopulmonary bypass. Ann Thorac Surg 2000;69(4):1198-204.

28. Al-Ruzzeh S, George S, Bustami M, Wray J, Ilsley C, et al. Effect of off-pump coronary artery bypass surgery on clinical, angiographic, neurocognitive, and quality of life outcomes: randomised controlled trial. BMJ 2006;332(7554):1365.

29. Lachmann B. Open up the lung and keep the lung open. Intensive Care Med 1992; 18(6):319-21.

30. Verbrugge SJ, Sorm V, Lachmann B. Mechanisms of acute respiratory distress syndrome: role of surfactant changes and mechanical ventilation. J Physiol Pharmacol 1997;48(4)537-57.

31. Fan E, Del Sorbo L, Goligher EC, Hodgson CL, Munshi L, Walkey AJ, et al. An Official American Thoracic Society/European Society of Intensive Care Medicine/Society of Critical Care Medicine Clinical Practice Guideline: Mechanical Ventilation in Adult Patients with Acute Respiratory Distress Syndrome. Am J Respir Crit Care Med 2017:195(9):1253-1263.

32. Gattinoni L, Quintel M. How ARDS should be treated. Crit Care. 2016,20:86.

33. Gattinoni L, Tonetti T, Cressoni M, Cadringher P, Herrmann P, Moerer O, et al. Ven- tilator-related causes of lung injury: the mechanical power. Intensive Care Med 2016;42(10):1567-1575

34. Fan E, Checkley W, Stewart TE, Muscedere J, Lesur O, Granton JT, et al. Complications from recruitment maneuvers in patients with acute lung injury: secondary analysis from the lung open ventilation study. Respir Care 2012;57(11):1842-9.

35. Fan E, Wilcox ME, Brower RG, Stewart TE, Mehta S, Lapinsky SE, et al. Recruitment maneuvers for acute lung injury: a systematic review. Am J Respir Crit Care Med 2008; 178(11):1156-63.

36. Cavalcanti $A B$, Suzumura $E A$, Laranjeira $L N$, Paisani DM, Damiani $L P$, Guimarães $H P$, et al. Effect of Lung Recruitment and Titrated Positive End-Expiratory Pressure (PEEP) vs LOW PEEP on Mortality in Patients With Acute Respiratory Distress Syndrome: A Randomized Clinical Trial. JAMA 2017;318(14):1335-1345.

37. Sahetya SK, Brower RG. Lung Recruitment and Titrated PEEP in Mod erate to Severe ARDS: Is the Door Closing on the Open Lung? JAMA2017;318(14):1327-1329.

38. Costa Leme A, Hajjar LA, Volpe MS, Fukushima JT, De Santis Santiago RR, Osawa EA, et al. Effect of Intensive vs Moderate Alveolar Recruitment Strategies Added to Lung-Protective Ventilation on Postoperative Pulmonary Complications: A Randomized Clinical Trial. JAMA 2017:317(14):1422-1432.

39. Magnusson L, Zemgulis V, Tenling A, Wernlund J, Tyden H, Thelin S, et al. Use of a vital capacity maneuver to prevent atelectasis after cardiopulmonary bypass: an experimental study. Anesthesiology 1998;88(1):134-42.

40. Loeckinger A, Kleinsasser A, Lindner KH, Margreiter J, Keller C, Hoermann C. Continuous positive airway pressure at $10 \mathrm{~cm} \mathrm{H(2)O} \mathrm{during} \mathrm{cardiopulmonary} \mathrm{bypass}$ improves postoperative gas exchange. Anesth Analg 2000;91(3):522-7.

41. John LC, Ervine IM. A study assessing the potential benefit of continued ventilation during cardiopulmonary bypass. Interac Cardiovasc Thorac Surg 2008;7(1):14-7. 


\section{va a impedir que veamos a nuestros nietos?}

zLa vejez nos

\section{$80 \%$}

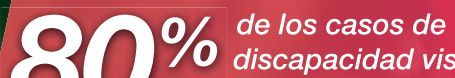 \\ $80 \%$}

\section{ViveMejor.com.ar}




\section{HIPERTENSIÓN PULMONAR TROMBOEMBÓLICA CRÓNICA EN LA ERA DE LA MÍNIMA INVASIVIDAD}

\section{CHRONIC THROMBOEMBOLIC PULMONARY HYPERTENSION IN THE ERA OF MINIMAL INVASIVENESS}

\section{SEBASTIÁN SRUR ${ }^{1}$}

\section{RESUMEN}

La hipertensión pulmonar tromboembólica crónica (HPTC) es un tipo de hipertensión pulmonar que se caracteriza por la estenosis de los vasos pulmonares con material trombótico de resolución incompleta, así como por un grado variable de arteriopatía esclerofibrótica. La importancia de su correcto y precoz diagnóstico radica en que posee un tratamiento potencialmente curativo, la tromboendarterectomía pulmonar. Sin embargo un tercio de los pacientes no se operan por el elevado riesgo del procedimiento o porque el proceso tromboembólico resulta técnicamente inoperable; es en este grupo de pacientes en que cobran importancia otras alternativas terapéuticas, como la angioplastia con balón a las arterias pulmonares y el tratamiento farmacológico dirigido. Tanto la angioplastia con balón como las terapias farmacológicas resultan alternativas prometedoras. Nuevas investigaciones son fundamentales para definir mejor el rol de éstas en la HPTC.

Palabras clave: hipertensión pulmonar; embolia pulmonar; angioplastia con balón; endarterectomía.

\section{ABSTRACT}

Chronic thromboembolic pulmonary hypertension (CTEPH) is a type of pulmonary hypertension that is characterized by stenosis of the pulmonary vessels with incompletely resolved thrombotic material, as well as by a variable degree of sclerofibrotic arteriopathy. The importance of its correct and early diagnosis is that it has a potentially curative treatment, pulmonary thromboendarterectomy. However, one third of patients are not operated due to the high risk of the procedure or because the thromboembolism is technically inoperable; it is precisely in this group of patients, in which other therapeutic alternatives, such as balloon angioplasty to the pulmonary arteries and managed pharmacological treatment, become important. Both balloon angioplasty and pharmacological therapies are promising alternatives. New research is essential to better define the role of these in the CTEPH.

Keywords: pulmonary hypertension; pulmonary embolism; balloon angioplasty; endarterectomy.

REVISTA CONAREC 2018;33(146):227-238 | DOI:10.32407/RCON/2018146/0227-0238

\section{INTRODUCCIÓN}

La hipertensión pulmonar tromboembólica crónica (HPTC) es un tipo de hipertensión pulmonar (HTP) que se caracteriza por la obstrucción trombótica de pequeños y grandes vasos arteriales pulmonares y un grado variable de arteriopatía. El remodelado vascular esclerofibrótico conlleva a un aumento de la resistencia vascular pulmonar (RVP). De no mediar tratamiento, este proceso se asocia a una elevada morbimortalidad, principalmente debido a la insuficiencia cardíaca derecha ${ }^{1}$.

El tratamiento quirúrgico -la tromboendarterectomía pulmonar (TEAP) - es el gold standard terapéutico de esta patología. Sin embargo, más de un tercio de los pacientes que tienen indicación de cirugía no se operan por el elevado riesgo quirúrgico o porque el proceso tromboembólico resulta técnicamente inoperable. Vale la pena recordar que otro porcentaje importante de pacientes no llega a la cirugía por falta de acceso a ella. Es en estos casos donde cobran importancia otras alternativas terapéuticas, como la angioplastia con balón de

1. Médico Residente de Cardiología. Sanatorio de la Trinidad-Mitre. Ciudad Autónoma de Buenos Aires, Rep. Argentina.

$\triangle$ Correspondencia: Dr. Sebastián Srur. Bmé. Mitre 2553. C1039AAO CABA, Rep. Argentina.sebastiansrur@gmail.com

Los autores declaran no tener conflictos de intereses.

Recibido: 27/10/2017| Aceptado: 10/05/2018 las arterias pulmonares (ABAP) y los tratamientos farmacológicos dirigidos. La anticoagulación crónica es el denominador común a todas estas estrategias terapéuticas².

Los objetivos de la siguiente revisión son, en primer lugar, definir y encuadrar epidemiológicamente a la HPTC; en segundo lugar, comprender su fisiopatología; y finalmente analizar las nuevas terapéuticas actualmente disponibles para su tratamiento, intentando abordar esta lectura desde una mirada práctica, orientada hacia la toma de decisiones del cardiólogo clínico frente a este nuevo mar de alternativas.

\section{MATERIALES Y MÉTODOS}

La búsqueda bibliográfica fue realizada principalmente a partir de los motores de búsqueda de PubMed, SCIELO, LILACS y Cochrane, utilizando palabras claves con lenguaje MeSH (en el caso de PubMed): pulmonary hypertension, thromboembolism, pulmonary embolism, venous thromboembolism, angioplasty, thrombectomy, bosentan, riociguat, anticoagulants, blood coagulation.

Para la obtención de los textos completos se acudió a la biblioteca de la Sociedad Argentina de Cardiología y de la Facultad de Medicina de la Universidad de Buenos Aires. Luego de esta primera búsqueda se realizaron otras, basadas en el material encontrado.

Se delimitó la selección mayoritariamente a artículos publicados entre 1900 y septiembre de 2016. Se prefirieron las publicaciones originales, tanto básicas como clínicas, los metaanálisis y las revisiones sistemáticas. 


\section{GENERALIDADES DE LA HIPERTENSIÓN PULMONARTROMBOEMBÓLICA CRÓNICA}

\section{DEFINICIÓN}

Fisiopatológicamente, la HPTC es una forma de HTP determinada por la estenosis de pequeñas y grandes arterias pulmonares por un trombo de resolución incompleta, así como de un grado variable de vasculopatía esclerofibrótica. Este cambio en las propiedades histológicas y estructurales del árbol vascular pulmonar incrementan la RVP, con el consiguiente aumento de la poscarga del ventrículo derecho, la derivación de flujo sanguíneo hacia otros lechos pulmonares y un aumento de la fuerza de cizallamiento (o shear stress) local'

En la práctica clínica, la HPTC se establece a partir de la presencia simultánea de cada una de las siguientes condiciones:

- una presión arterial media pulmonar (PAPm) $\geq 25 \mathrm{mmHg}$ y una presión de enclavamiento $(P C P) \leq 15 \mathrm{mmHg}$, diagnosticadas mediante cateterismo cardíaco derecho,

- la presencia de trombo pulmonar evidenciado a partir de un defecto de perfusión por centellografía ventilación/perfusión,

- la confirmación de la presencia de trombo pulmonar evidenciado a partir de otros métodos por imágenes (tomografía computada, resonancia magnética o angiografía pulmonar),

- reunidos todos estos elementos en un paciente tratado durante $\geq 3$ meses con anticoagulación efectiva (este último punto, enfatizando en el carácter crónico del cuadro)².

\section{EPIDEMIOLOGÍA}

La HPTC es una enfermedad poco frecuente, producto de una evolución tórpida luego del tromboembolismo de pulmón (TEP) agudo. Menos del 10\% (entre 0,4 y 9,1\%) de los pacientes con TEP agudo evolucionan a formas crónicas ${ }^{2-4}$.

La ausencia de antecedentes claros de TEP o trombosis venosa profunda (TVP) no debería descartar a priori la presencia de esta entidad. Se estima que aproximadamente el 25\% de los pacientes con HPTC no cuentan con un antecedente de TEP -al menos no registrado en su historia clínica- y cerca del 50\% ni siquiera ha presentado episodios de TVP5

La prevalencia e incidencia anual reales de HPTC son datos desconocidos, si bien registros europeos sugieren que la incidencia actual es de aproximadamente 5 casos por millón de habitantes por año en la población general ${ }^{6,7}$. Según el registro español, la prevalencia es de 8,9 casos por millón de habitantes ${ }^{8}$.

En la República Argentina se publicó en el año 2012 el registro HINPULSAR, el cual incluyó a 422 pacientes con HTP9. La prevalencia relativa de cada uno de los grupos de HTP fue la siguiente: grupo | 29,4\%, grupo || 52,8\%, grupo I|| 10\%, grupo IV (HPTC) 6,4\% y grupo V 1,4\%. Si bien estos datos se encuentran lejos de arrojar una luz sobre la prevalencia de la HPTC en la población general de nuestro país, ilustran claramente su baja frecuencia dado que incluso entre los pacientes con HTP, los que presentan HPTC no alcanzaron ni e $10 \%$ de los pacientes incluidos. Actualmente se encuentra en curso un nuevo registro de esta patología, el estudio RECOPILAR (I Registro Colaborativo de Hipertensión Pulmonar en Argentina) que quizás pueda aportar más información sobre nuestra epidemiología local ${ }^{10}$.
Se especula con que la prevalencia e incidencia reales de la HPTC podrían ser mayores que las estimadas, dado que se trata de una condición frecuentemente subdiagnosticada por la comunidad médica. En el registro argentino HINPULSAR se detectó una franca subutilización de exámenes complementarios considerados como de referencia para el diagnóstico de esta patología. Por ejemplo, el centellograma ventilación/perfusión solo se utilizó en un 33\% de los casos y la angiotomografía en menos del $5 \%$. De manera similar, en un registro norteamericano reciente que buscaba enrolar a individuos con hipertensión arterial pulmonar (HAP) primaria, el 43\% de los pacientes incluidos no fueron estudiados apropiadamente mediante el método diagnóstico de elección -el centellograma ventilación/perfusiónpara descartar HPTC, poniendo en tela de juicio el correcto diagnóstico de HAP primaria $2,11,12$.

El grado de subdiagnóstico de esta patología fue ponderado recientemente por Kim ${ }^{13}$. A partir de las estimaciones norteamericanas de una incidencia de TEP de 600.000 casos por año y una progresión del 0,57\% a HPTC, por lo que habría 3.420 casos de HPTC evolucionados a partir de TEP por año ${ }^{14,15}$. Si a su vez se contemplara que 30\% de los pacientes con HPTC no presentaron antecedentes registrados de TEP habría que considerar 1.466 casos anuales adicionales. Estos cálculos concluirían en aproximar que existen 4.886 nuevos casos de HPTC por año en los EEUU. En este punto, vale la pena preguntar: ¿Cuántas TEAP se realizan anualmente en dicho país? Tan solo entre 300 y 400 procedimientos por año. Por consiguiente, menos del $8 \%$ de los nuevos casos por año de HPTC reciben el tratamiento de elección. La brecha en esta discrepancia habla por sí sola.

\section{FISIOPATOLOGÍA}

La HPTC fue descripta por primera vez en 1929 por el investigador norteamericano Barnes, quien registró el primer caso de un sobreviviente al episodio agudo de TEP que evolucionó a una forma crónica de la patología, con desarrollo de HTP y, finalmente, falla ventricular derecha ${ }^{16}$.

Si bien existe conocimiento de esta patología desde hace casi un siglo, actualmente continúan sin esclarecerse por completo los mecanismos subyacentes implicados en su desarrollo. El modelo fisiopatológico actual se centra en la resolución incompleta del trombo generado a partir de un evento tromboembólico pulmonar (Figura 1) En el TEP agudo la oclusión de las arterias pulmonares con material trombótico (principalmente eritrocitos y fibrina) podría presentar dos historias naturales muy distintas: por una parte podría progresar hacia la resolución del trombo por acción del sistema fibrinolítico en primera instancia y luego hacia la recanalización mediante la neovascularización; o bien podría progresar hacia la resolución del trombo por acción del sistema fibrinolítico; o alternativamente podría evolucio nar hacia la formación de cicatrices vasculares en forma de bandas o membranas. La persistencia de estas obstrucciones vasculares deriva el flujo sanguíneo hacia vasos en paralelo que no se encuentran obstruidos y, por consiguiente, presentan menor resistencia. En dichos territorios el flujo sanguíneo local aumenta, incrementando a su vez la presión lateral y la fuerza de cizallamiento (o shear stress). Estos cambios hemodinámicos generan disfunción endotelial y remodelado microvascular. La progresión crónica de estos fenómenos aumen- 


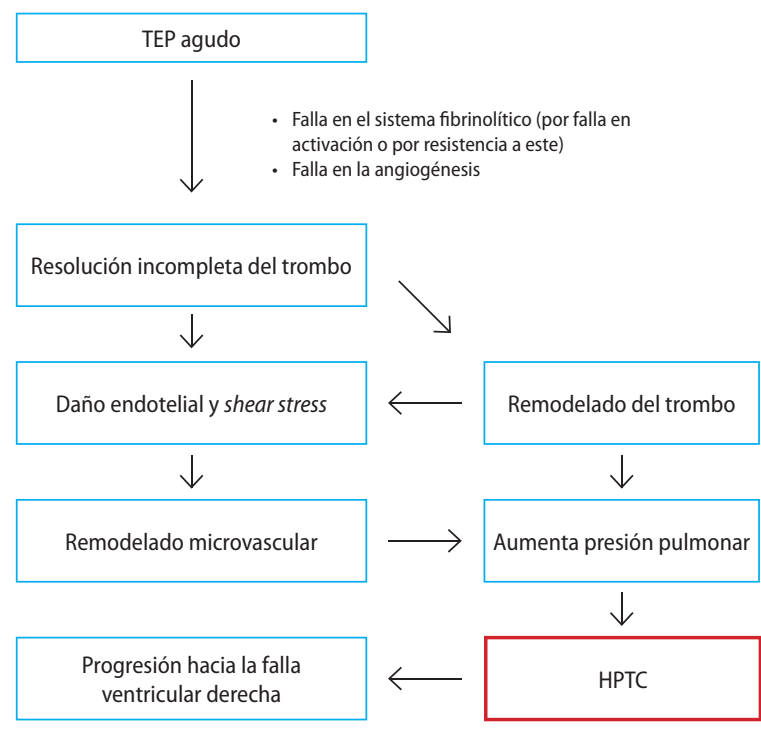

Figura 1. Fisiopatología de la hipertensión pulmonar tromboembólica crónica. Luego del TEP agudo, un pequeño porcentaje evoluciona a HPTC. Se cree que esto se debe principalmente a la łesolución incompleta del trombo, ya sea por falla en activación o por resistencia al sistema fibrinolítico. También se ha propuesto la falla en la angiogénesis como mecanismo. El por qué fallan estos mecanismos sigue sin estar aclarado, aunque se ha visto relación entre la severidad del TEP y la progresión a HPTC, además de otros factores que se han asociado en gran medida con esta evolución. La persistencia del material trombótico genera daño endotelial y shear stress, principalmente por aumento del flujo sanguíneo a vasos de menor resistencia. Esto genera daño endotelial y remodelado microvascular, con arteriopatía plexiforme, similar a la observada en la HAP primaria. El trombo y la arteriopatía sobreimpuesta generan aumento de la presión pulmonar, y, por lo tanto, HPTC. El aumento de la poscarga del ventrículo derecho, finalmente, lleva hacia la falla ventricular derecha, principal causa de muerte de estos pacientes. HPTC: hipertensión pulmonar tromboembólica crónica. TEP: tromboembolismo pulmonar. Modificado de Modifcado de Morris, TA. Why acute pulmonary embolism becomes chronic thromboembolic pulmonary hypertension: clinical and genetic insights. Curr Opin Pulm Med 2013;19(5):422-9.

ta la RVP. Esta sucesión de hechos explica por qué la HPTC es una enfermedad progresiva, que avanza aún en ausencia de nuevos eventos tromboembólicos ${ }^{17-19}$.

El aumento de la RVP representa un incremento en la poscarga del ventrículo derecho, poniéndose en marcha mecanismos adaptativos como la hipertrofia y dilatación del mismo. La disfunción diastólica, inicialmente, y luego la disfunción sistólica, conducen a una progresiva insuficiencia cardíaca derecha, la principal limitante en la sobrevida de estos pacientes?

Los elementos necesarios para el desarrollo de HPTC son por consiguiente la persistencia de un trombo residual y el desarrollo de presiones pulmonares arteriales elevadas, presentándose ambas aún después de un período considerable de tratamiento anticoagulante eficaz. El período durante el cual dicho proceso se desarrolla puede requerir meses o incluso años (habitualmente entre 6 meses y 2 años) durante los cuales el paciente puede encontrarse asintomático u oligosintomático. El prolongado período de latencia entre el evento primario gatillo y el desarrollo de HPTC propiamente dicha, junto con una presentación clínica inicialmente insidiosa complejizan la detección de este cuadro. Es solamente mediante la presencia conjunta de estas condiciones -necesarias, pero no suficientes cada una por su cuenta- que se desarrollaría el cuadro de HPTC, constituyendo las bases de su definición clínica ${ }^{4}$.

Una de las primeras grietas en la hipótesis de la resolución incomple-

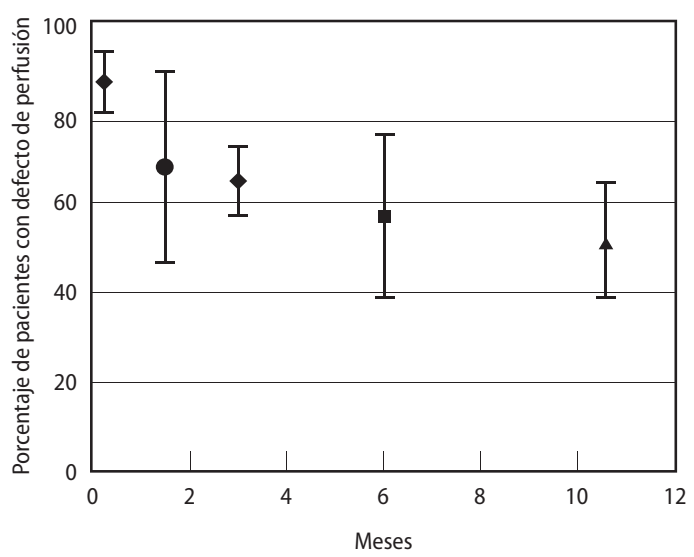

Figura 2. Proporción de pacientes con trombosis residual en los 12 meses posteriores a un episodio de tromboembolismo pulmonar agudo en un metaanálisis de cohortes prospectivas. La prevalencia de pacientes con trombosis residual permaneció elevada ( $\geq 50 \%)$ incluso 12 meses luego del evento, indicando su baja asociación con la progresión al infrecuente cuadro de hipertensión pulmonar tromboembólica crónica. Modificado de Nijkeuter M, Hovens MM Davidson BL, Huisman MV. Resolution of thromboemboli in patients with acute pulmonary embolism: a systematic review. Chest 2006;129(1):192-7.

ta del trombo como modelo fisiopatológico hegemónico radica en que la sola presencia de trombo residual no garantiza la evolución a HPTC ${ }^{20}$. En un metaanálisis de cohortes prospectivas realizado por Nijkeuter et al., casi un 90\% de los pacientes con TEP agudo persistía con un defecto de perfusión en la centellografía ventilación/perfusión luego de 1 semana del evento agudo. A los 11 meses, más del $50 \%$ presentaba dicho defecto de perfusión (Figura 2). A pesar de ello, la tasa de progresión de TEP a HPTC es cinco a diez veces menor a estas cifras en la mayoría de las series temporales (0,4-9,1\%), indicando un bajo valor predictivo positivo de este hallazgo ${ }^{21}$

El reconocimiento de que a partir de un episodio de TEP agudo solamente algunos pacientes evolucionarían a HPTC (mientras que la gran mayoría no lo hace) motivó la búsqueda de otros factores de riesgo que pudieran predecir el riesgo de evolucionar hacia HPTC. En una cohorte prospectiva de 78 pacientes que hubieran presentado un episodio de TEP agudo se demostró que una presión sistólica de la arteria pulmonar (PSAP) al ingreso $\geq 50 \mathrm{mmHg}$, odds ratio (OR) de 3,3 e intervalo de confianza del 95\% (IC95\%), y una edad $\geq 70$ años (OR=4,1; IC95\%) se relacionaban con un riesgo tres a cuatro veces mayor de evolucionar a HPTC. Por el contrario, llamativamente, la presencia de disfunción ventricular derecha no se encontró relacionada con una evolución a HPTC, recobrando la gran mayoría de los pacientes su función ventricular derecha entre 8 y 30 días posteriores al evento agudo ${ }^{22}$.

Otras variables que se han relacionado con este tipo de evolución son los grandes defectos de perfusión y la enfermedad tromboembólica idiopática ${ }^{23}$. Incluso algunas condiciones infrecuentes también se han relacionado con el desarrollo de HPTC: la esplenectomía previa, en la que se ha postulado que el aumento de los eritrocitos anormales no filtrados por el bazo pueden depositarse en la circulación pulmonar; las derivaciones ventriculoauriculares para el tratamiento de la hidrocefalia, en las que se ha postulado la infección estafilocócica como probable desencadenante; las enfermedades inflamatorias crónicas (p.ej. osteomielitis, enfermedad inflamatoria intestinal) y la pertenencia a un grupo sanguíneo distinto al $0^{24}$. 


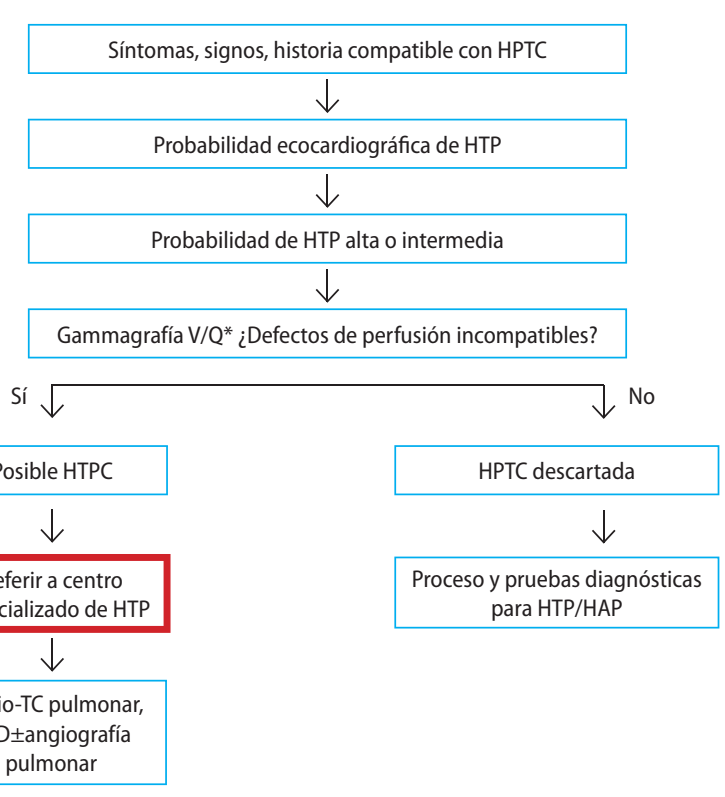

Figura 3. Algoritmo diagnóstico de la hipertensión pulmonar tromboembólica crónica. * Presenta sensibilidad cercana al 100\%. La angiotomografía por sí sola puede no lograr el diagnóstico de HPTC. HTP: hipertensión pulmonar. HAP: hipertensión arterial pulmonar. Gammagrafía V/Q: gammagrafía ventilación/perfusión. HPTC: hipertensión pulmonar tromboembólica crónica. TC: tomografía computada. CCD: cateterismo cardiaco de recho. Modificado de Galiè N, Humbert M, Vachiery JL, Gibbs S, Lang I, Torbicki A et al. 2015 ESC/ERS Guidelines for the diagnosis and treatment of pulmonary hypertension. Eur Heart J 2016;37(1):67-119.

Las bases moleculares de una resolución incompleta de un trombo pulmonar y la posterior evolución a HPTC han presentado algunos avances en los últimos años, pudiendo identificar algunas moléculas responsables, vinculadas fundamentalmente con los procesos de hemostasia y fibrinólisis. La fibrinólisis es un proceso complejo en el que intervienen, entre otras moléculas, el plasminógeno (que se activa a plasmina y actúa sobre la fibrina), el factor activador del plasminógeno tisular (t-PA) y el factor inhibidor del activador del plasminógeno (PAl-1). Los mecanismos fisiopatológicos propuestos para la resolución incompleta del trombo se basan en una inadecuada actividad del sistema fibrinolítico ${ }^{25}$.

Existe evidencia que sustenta que una disminución de la activación del sistema fibrinolítico podría ser responsable de la resolución incompleta del trombo. En un estudio realizado en perros a los que se les indujo experimentalmente TEP, la administración de ácido tranexámico (p. ej., un inhibidor del sistema fibrinolítico) impidió la resolución del trombo y condujo a un aumento de las presiones pulmonares. En las autopsias posteriores se encontró material trombótico organizado, similar a la histopatología de la HPTC ${ }^{26}$. Lang et al. evaluaron por inmunohistoquímica a las piezas histopatológicas de pacientes sometidos a TEAP. Las zonas con mayor contenido trombótico presentaban mayor expresión de PAI-1 en las células endoteliales y de músculo liso, lo que sugiere un aumento de la inhibición del sistema fibrinolítico como causa del desarrollo de la lesión ${ }^{27}$.

Una hipótesis alternativa es que el trombo en sí mismo ofrezca mayor resistencia al sistema fibrinolítico. En este sentido, Morris et al. demostraron que una gran proporción de los pacientes con HPTC presentan una molécula de fibrina que es más resistente a la lisis por plasmina en comparación con sujetos sanos ${ }^{28}$.
Asimismo, se ha postulado como factor causal de la incompleta resolución del trombo a la falla en la angiogénesis. Ante la presencia de material trombótico, las células endoteliales, los leucocitos y las plaquetas secretan enzimas, factores quimiotácticos y factores proangiogénicos (p. ej., la interleuquina-8, el factor de crecimiento vascular y el factor de crecimiento fibroblástico) que contribuirían con la angiogénesis local, pudiendo de este modo favorecer la recanalización de la arteria obstruida y dar origen a nuevos vasos en paralelo que permitan el paso del caudal local18,23. En un estudio histopatológico de muestras de pacientes con HPTC sometidos a TEAP, se observó que las lesiones más recanalizadas presentaban mayor actividad angiogénica que en aquellos con más lesiones trombóticas ${ }^{29}$.

A modo de resumen, si bien los principales elementos fisiopatológicos de la HPTC son la persistencia de un trombo residual y el desarroIlo de presiones pulmonares arteriales elevadas refractarios a una anticoagulación eficaz, habría numerosos otros factores subyacentes y colindantes vinculados con el desarrollo de esta patología.

\section{SINTOMATOLOGÍA Y DIAGNÓSTICO}

Al igual que en otras formas de HTP, la HPTC presenta a la disnea progresiva como síntoma más frecuente. Habitualmente los pacientes que presentaron un evento de TEP agudo sintomático atraviesan un período de "luna de miel" variable, en donde se encuentran asintomáticos u oligosintomáticos. Este período suele durar de 6 meses a 2 años, período a partir del cual comienzan a manifestar disnea a menores esfuerzos ${ }^{1,30}$. Adicionalmente, los pacientes pueden presentar fatiga, síncope y signosintomatología de falla ventricular derecha. La hemoptisis se manifiesta en un 5\% de los pacientes y se encuentra relacionada con la dilatación de las arterias bronquiales ${ }^{31}$.

La HPTC debe ser sospechada ante todo paciente con HTP de causa no aclarada, independientemente de la presencia o no de antecedentes de TEP, ya que, como se ha mencionado, aproximadamente un tercio de los casos pueden desarrollar este cuadro aún sin haber presentado un evento tromboembólico clínico. Por su clínica insidiosa y su baja sospecha, el tiempo medio de diagnóstico de esta patología es de 14 meses luego del inicio de los síntomas 5 .

Según el algoritmo propuesto en el año 2015 por la guía europea de hipertensión pulmonar (Figura 3), ante la sospecha de HTP, el primer paso consiste en realizar un ecocardiograma Doppler². Si se obtienen hallazgos ecocardiográficos compatibles con HTP, posteriormente se debe realizar una centellografía ventilación/perfusión. En un reporte realizado por Tunariu et al., la centellografía ventilación/perfusión presentó una sensibilidad cercana al 100\%, por lo que un resultado negativo excluiría virtualmente la posibilidad de HPTC. A modo de comparación, según este reporte la angiotomografía presentó una sensibilidad de solo el 51\% para el diagnóstico de esta entidad ${ }^{32}$.

El paso siguiente recomendado por estas guías es la realización de un cateterismo cardíaco derecho, con medición de parámetros hemodinámicos. El diagnóstico específico de la patología se realiza mediante la visualización de las obstrucciones a nivel de la vasculatura pulmonar, la presencia de estenosis "en anillo" y de bandas de estenosis (webs) por angiografía pulmonar, angiotomografía pulmonar multicorte o resonancia magnética (RM). Además, estos méto- 


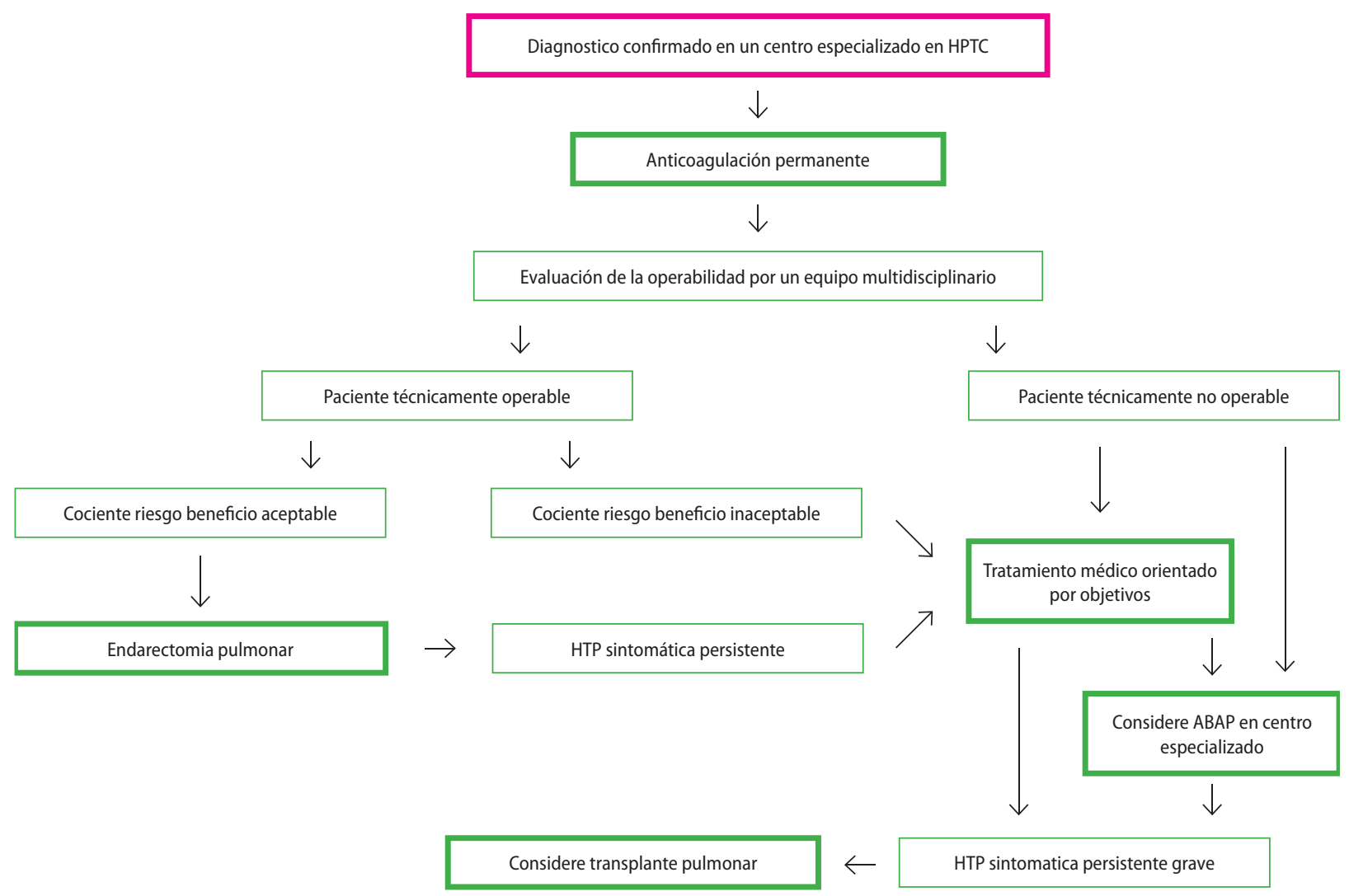

Figura 4. Algoritmo terapéutico de la hipertensión pulmonar tromboembólica crónica. Una vez hecho el diagnóstico, todos los pacientes deben estar anticoagulados. Por ser la TEAP el tratamiento de elección, todos los pacientes deben ser evaluados para recibirla por un equipo multidisciplinario. Si el paciente es técnicamente operable, con un cociente riesgo/beneficio aceptable debe realizarse la TEAP. En caso de que el paciente sea técnicamente inoperable o que tengan un cociente riesgo/beneficio inaceptable, las alternativas son el tratamiento médico (el único aprobado para este uso es el riociguat) y la ABAP en centros especializados. En casos de HTP persistente sintomática grave, considerar el trasplante pulmonar. HTP: hipertensión pulmonar. HPTC: hipertensión pulmonar tromboembólica crónica. TEAP: tromboendarterectomía pulmonar. ABAP: angioplastia con balón a las arterias pulmonares. Modificado de: Galiè N, Humbert M, Vachiery JL, Gibbs S, Lang I, Torbicki A, et al. 2015 ESC/ERS Guidelines for the diagnosis and treatment of pulmonary hypertension. Eur Heart J 2016;37(1):67-119.

dos nos aportan información sobre el tipo de enfermedad predominante (proximal o distal), la posibilidad de resección mediante TEAP y el compromiso de estructuras adyacentes (p.ej. la obstrucción del tronco de la arteria coronaria izquierda por la dilatación de la arteria pulmonar) ${ }^{30}$.

Tal como se mencionó previamente, el diagnóstico definitivo de HPTC requiere la presencia de los siguientes hallazgos luego de un período de anticoagulación efectiva de al menos 3 meses: cateterismo cardíaco derecho con PAPm $\geq 25$ y PCP $\leq 15 \mathrm{mmHg}$, defecto en la perfusión mediante centellografía ventilación/pulmonar y signos compatibles con HPTC en estudios por imágenes tales como la angiografía pulmonar, la angiotomografía pulmonar o la RM².

\section{TERAPÉUTICAS: ESTÁNDAR Y NUEVAS PERSPECTIVAS}

Ante el correcto diagnóstico de HPTC, todos los pacientes deberían recibir tratamiento anticoagulante prolongado con antagonistas de la vitamina K (con un objetivo de RIN entre 2 y 3) o con heparinas (tanto las no fraccionadas como las de bajo peso molecular). No hay evidencia para recomendar el uso de nuevos anticoagulantes orales ${ }^{12}$. Además de la anticoagulación, la cirugía es el tratamiento de elección mediante la TEAP (Figura 4).

\section{TRATAMIENTO QUIRÚRGICO}

La HPTC es la única forma de HTP en la que el tratamiento quirúrgico es potencialmente curativo, por lo que es de elección y se encuentra como recomendación de grado I con nivel de evidencia C, tanto en la guía europea del año 2015 como en la norteamericana del año $2009^{2,33}$.

Previo al advenimiento de la TEAP, el pronóstico de la HPTC era ominoso: según un estudio publicado por Riedel et al. en 1982, la supervivencia media era de 6,8 años, y si la PAPm excedía los 50 mmHg la mortalidad a los 3 años era aproximadamente del $90 \%{ }^{34}$. Actualmente, según el registro europeo de HPTC, en los pacientes operados la supervivencia a 3 años es del $89 \%$ contra un $70 \%$ en los pacientes no operados. Si bien los autores reportan que esta diferencia es estadísticamente significativa $(p<0,0001)$, habiendo una disminución del riesgo relativo (RR) de muerte del 67\% en los pacientes operados, es necesario recordar que se trata de un registro, y por consiguiente de un estudio observacional de cohortes, en donde los grupos no necesariamente son comparables entre sír. Habiendo dicho eso, frente a los impactantes resultados clínicos de la TEAP ante un cuadro de tan mal pronóstico inicial, por motivos éticos no existen actualmente ensayos clínicos aleatorizados que comparen la estrategia quirúrgica contra el tratamiento médico. Si bien son escasos los datos de seguimiento a largo plazo, existe un estudio observacional en pacientes post-TEAP en los que la supervivencia luego de 5 años es del $84 \% \%^{35}$. 
Tabla 1. Resultados de tromboendarterectomías pulmonares realizadas en centros expertos.

\begin{tabular}{lccccc} 
& $\begin{array}{c}\text { Número de } \\
\text { procedimientos }\end{array}$ & $\begin{array}{c}\text { RVP media } \\
\text { previa a TEAP }\end{array}$ & $\begin{array}{c}\text { RVP media } \\
\text { posterior a TEAP }\end{array}$ & $\begin{array}{c}\text { Mortalidad } \\
\text { intrahospitalaria }\end{array}$ & Supervivencia a largo plazo \\
\hline Madani et al (37) & 500 & 719 & 253 & $2,2 \%$ & $82 \%$ a 5 años, $75 \%$ a 10 años. \\
\hline Corsico et al (35) & 157 & 1140 & 327 & $11,5 \%$ & $84 \%$ a 5 años \\
\hline Berman et al (38) & 411 & 693 & 308 & $5,4 \%$ & $91 \%$ al año, 88\% a los 3 años. \\
\hline Favaloro et al (41) & 41 & 857 & 247 & $15 \%$ & $85 \%$ al año, $75 \%$ a los 5 años, $72 \%$ a los 10 años. \\
\hline
\end{tabular}

RVP: medida en dyn.S.C.m ${ }^{-5}$. TEAP: tromboendarterectomía pulmonar.

La TEAP consiste en la realización de una verdadera tromboendarterectomía pulmonar bilateral, incluyendo la capa íntima y parte de la media. El objetivo de esta es disminuir las resistencias vasculares pulmonares, disminuir la poscarga del ventrículo derecho y mejorar la relación ventilación/perfusión ${ }^{36}$. Luego del procedimiento, la mejoría hemodinámica es significativa, logrando llevar los valores cercanos a la normalización. Según un estudio de 1.000 pacientes de la Universidad de California en San Diego (UCSD), el centro con más experiencia en la realización de éste procedimiento, 3 meses luego de la TEAP hay una reducción significativa de la RVP en un 70\% (desde 861,2 a 294 dyn.s.cm ${ }^{-5}$ ) y de la PAPm en un $40 \%$ (desde $46,1 \pm 11$ a $28,7 \pm 10 \mathrm{mmHg}$ ), y un aumento significativo del índice cardíaco en un $25 \%$ (desde 2 a 2,5 I/min/m²). Hubo una mejoría en la clase funcional (CF) de los pacientes, como también en la distancia recorrida durante la caminata de 6 minutos (DR6) ${ }^{37}$. Si bien existen pocos estudios de seguimiento hemodinámico a largo plazo, habría evidencia para sustentar la hipótesis de que la mejoría clínica pareciera mantenerse. En una cohorte prospectiva de Corsico et al. se observó que el 70\% de reducción de la RVP media a los 3 meses de la cirugía (desde 1.140 a $327 \mathrm{dyn} \cdot \mathrm{s} \cdot \mathrm{cm}^{-5}$ ) se mantenía a los 4 años post-TEAP (339 dyn.s. $\left.\mathrm{cm}^{-5}\right)^{35}$. En la Tabla 1 se resumen los resultados intrahospitalarios y a largo plazo de las TEAP realizadas en centros con elevada experiencia ${ }^{35,37,38,41}$.

La intervención quirúrgica en sí misma se realiza mediante esternotomía mediana, con apoyo de circulación extracorpórea e hipotermia profunda $\left(\leq 20^{\circ} \mathrm{C}\right)$. Se establecen períodos de parada circulatoria en hipotermia profunda de hasta 20 minutos de duración, alternados con períodos de reperfusión de 5 minutos. Habitualmente se completa la TEAP con un tiempo acumulado de parada circulatoria en cada arteria pulmonar inferior a 20 minutos, pero en casos técnicamente dificultosos (como estenosis distales u oclusiones) puede llegar hasta 80 minutos, con numerosos períodos de paro/reperfusión ${ }^{39}$. Habitualmente se comienza realizando una arteriotomía en la arteria pulmonar derecha, luego se identifica el correcto plano de disección entre la íntima y la media, y se procede a continuar con la disección de la rama lobar, segmentaria y subsegmentaria comprometida. Si el plano elegido es muy profundo, el riesgo de perforación es alto; en cambio si es muy superficial, puede quedar material tromboembólico remanente. Luego de haber culminado con la arteria pulmonar derecha, se prosigue con la misma técnica a la arteria pulmonar izquierda ${ }^{40}$

La mortalidad intrahospitalaria del procedimiento está inversamente relacionada a la experiencia del centro. Según un registro europeo de HPTC, la mortalidad intrahospitalaria de un centro con menos de 10 procedimientos al año es de casi el doble de los que presentan más de 10 procedimientos anuales $(8,8 \text { vs. } 4,7 \%)^{6}$. Por este motivo, la guía europea de HTP (2015) considera que un centro es adecuado para la realización de este procedimiento cuando realice al menos 20 cirugías anuales con menos del 10\% de tasa de mortalidad ${ }^{2}$ Globalmente los resultados clínicos parecieran adecuados: la tasa de mortalidad intrahospitalaria según los últimos registros europeos es de 4,7\% y según el último registro de la UCSD es del 2,2\% $\%^{6,37}$.

Actualmente, en nuestro país la TEAP se realiza solo en tres centros privados y, recientemente, en un centro del ámbito público, el Hospital El Cruce "Dr Néstor C. Kirchner"; el único que posee más experiencia es el Hospital Universitario Fundación Favaloro, que en e 2011 publicó un trabajo que analiza retrospectivamente a 41 TEAP realizadas desde 1992 a 2010 ${ }^{41}$. La mejoría hemodinámica de los pacientes fue significativa (la PAPm disminuyó de 53 a 29 mmHg y la RVP de 857 a 245 dyn.S.cm ${ }^{-5}$, mientras que el índice cardíaco aumentó de 2,3 a $3 \mathrm{l} / \mathrm{min} / \mathrm{m}^{2}$ ). Los autores reportaron una mejoría de los pacientes, dado que en el período prequirúrgico el $90 \%$ presentaba disnea en CF III/IV según la escala de la New York Heart Association (NYHA) mientras que en el período posquirúrgico menos del 5\% de los sobrevivientes a la intervención presentaba disnea en CF III/IV. Sin embargo, son claramente manifiestos los sesgos de selección asociados a la afirmación previa: la mortalidad intrahospitalaria fue del 15\% (tres veces más que la media de mortalidad intrahospitalaria en las series europeas). A pesar de ello, los datos de seguimiento de mediano y largo plazo son alentadores: la supervivencia global a 1, 2, 5 y 10 años fue del $85 \%, 82 \%$, 75\% y $71 \%$, respectivamente.

La TEAP presenta complicaciones similares a cualquier cirugía cardíaca convencional. Sin embargo, dos de ellas son específicas de este procedimiento:

- Edema por reperfusión. Es la complicación del posoperatorio más frecuente. Según un registro europeo/canadiense ocurre en un 9,6\% de los casos. La reperfusión genera aumento de la permeabilidad de los vasos generando edema pulmonar. Es definido como PAFI (relación entre la presión arterial de oxígeno y la fracción inspirada de oxígeno) menor a 300 e infilitrados en la radiografía de tórax. En un 60\% de los pacientes se desarrolla tempranamente, en un 35\% dentro de las 48 hs y en un $5 \%$ luego de las 48 hs. El tratamiento consiste en la estimulación de la diuresis y la ventilación protectiva, mediante bajos volúmenes, alta frecuencia y alta PEEP (presión positiva al final de la espiración intrínseca). En caso de hipoxemia severa puede ser necesaria una membrana de oxigenación extracórporea (ECMO) $)^{35}$.

- Complicaciones neurológicas. Existe el potencial riesgo de daño neurológico durante los episodios de paro circulatorio con hipotermia profunda. Con la mejoría de la técnica, el riesgo se ha minimizado. El estudio PEACOG comparó la técnica tradicional (paro cir- 
culatorio) contra la perfusión cerebral anterógrada durante la TEAP, no hallándose diferencias significativas en la función cognitiva a los 3 meses y al año, por lo se considera el procedimiento tradicional un método seguro y es la elección ${ }^{35,42}$

La HTP residual continúa siendo la principal causa de morbilidad y mortalidad en el posoperatorio. No existe una definición única, aunque una de las más aceptadas es la propuesta por Skoro-Sajer y colaboradores, que la definieron como la persistencia de valores de PAPm $\geq 25 \mathrm{mmHg}$ y de RVP $\geq 400$ dyn.s.cm ${ }^{-5}$ luego de 1 año de la cirugía ${ }^{43}$. En un registro internacional de HPTC, afectó a un 16,7\% de los pacientes, aunque hay reportes que sugieren que podría llegar incluso al $30 \%$ 6,35

La principal causa de la HTP residual es el predominio de enfermedad distal microvascular.

La HTP residual está asociada al aumento de la mortalidad intrahospitalaria. Un estudio realizado por la UCSD demostró que la mortalidad intrahospitalaria es significativamente mayor en los pacientes con RVP $\geq 500$ dyn.s.cm ${ }^{-5}$ (31\%) comparado con aquellos que presentaban RVP $<500$ dyn $\cdot \mathrm{s} \cdot \mathrm{cm}^{-5}(0,9 \%)^{40}$.

Ante la persistencia de las presiones pulmonares elevadas, el tratamiento recomendado es el farmacológico. Como se verá más adelante, la única droga aprobada para este grupo de pacientes es el riociguat.

También se han hecho estudios pequeños de ABAP en este tipo de pacientes, con resultados satisfactorios, aunque no permiten sacar conclusiones al respecto ${ }^{44}$.

A pesar de que la TEAP es un procedimiento potencialmente curativo, según los registros solo el $60 \%$ de los pacientes es sometido a este procedimiento. Las razones por las cuales no son operados, según los registros, son: a) la inaccesibilidad quirúrgica de las lesiones (habitualmente distales); b) el disbalance entre el aumento de la RVP y las lesiones encontradas (por lo cual se sospecha un importante componente de enfermedad microvascular); c) edad avanzada y comorbilidades importantes; d) RVP $\geq 1200$ dyn.s. $\mathrm{cm}^{-5} ;$ e) otras ${ }^{6}$.

A diferencia de la cirugía cardíaca, no existen scores validados (como el STS o el EuroSCORE) para la TEAP. Tampoco existen guías establecidas que indiquen fehacientemente los criterios de inoperabilidad, por lo que la decisión recae en el equipo quirúrgico ${ }^{45}$.

Considerando el importante porcentaje de pacientes que continúan sin recibir el tratamiento adecuado, las distintas sociedades científicas enfatizan la necesidad de que el paciente sea evaluado por un equipo multidisciplinario especializado², e incluso, algunos autores sugieren en caso de negativa, una reevaluación por otro equipo ${ }^{30}$

A pesar de lo importante que es la adecuada evaluación preoperatoria, esta no debería diferir la cirugía en los pacientes operables. El tiempo hasta que se realiza la misma habitualmente se encuentra retrasado por varias razones, entre las que se encuentran la demora en el diagnóstico definitivo, el aumento del uso del tratamiento farmacológico como puente y la disponibilidad de los centros que realizan el procedimiento ${ }^{36}$. Es prioritario saber que el tratamiento farmacológico como puente a la cirugía no está probado y no debería retrasar el tiempo a la intervención quirúrgica.

\section{NUEVAS ALTERNATIVAS TERAPÉUTICAS}

Aproximadamente el $40 \%$ de los pacientes con HPTC no son candidatos a recibir la TEAP. Para casos inoperables se han propuesto dos tipos de tratamiento: la ABAP y el tratamiento médico dirigido.

Los resultados de los estudios que los avalan son prometedores, por lo que sus indicaciones cada vez son más amplias.

Al ser terapéuticas relativamente nuevas, se carece de estudios de seguimiento a largo plazo.

\section{ANGIOPLASTIA CON BALÓN A LAS ARTERIAS PULMONARES}

La ABAP es un procedimiento hemodinámico que consiste en la dilatación con un catéter-balón de las estenosis de las ramas de la arteria pulmonar.

La primera ABAP fue realizada en la década del 80 en pacientes pediátricos con estenosis o hipoplasia de la arteria pulmonar ${ }^{45}$ y desde 1988 es realizada en casos de HPTC catalogados como inoperables ${ }^{46}$. En 2001 Feinstein et al. publicaron el primer estudio de angioplastia pulmonar en 18 pacientes con HPTC no candidatos a cirugía. Si bien la mejoría hemodinámica y sintomática fue significativa, presentó severas complicaciones: 11 pacientes presentaron edema posreperfusión, 3 requirieron ventilación mecánica y un paciente falleció ${ }^{47}$.

Pese al mal resultado inicial, algunos centros, sobre todo en Japón, continuaron realizando ABAP, mejorando así la técnica y la estrategia, y logrando resultados satisfactorios.

El procedimiento se lleva a cabo mediante un acceso venoso, que puede ser a través de la vena yugular interna o de la vena femoral. El primero es el recomendado, por la mejor accesibilidad a la arteria pulmonar. A través de un introductor de 9 French se inserta un catéter guía de 6 French hacia la arteria pulmonar a tratar. Luego de realizar la angiografía pulmonar selectiva, se progresa el alambre guía de 0,014" a través de la lesión. Luego, con un catéter-balón de diámetro adecuado (1,5 a $10 \mathrm{~mm}$ ) se dilata el vaso con la lesión ${ }^{48,49}$.

Algunos centros que realizan el procedimiento se basan además en otros métodos por imagen como la ultrasonografía intravascular (IVUS) y la tomografía por coherencia óptica (OCT) $)^{50,51}$

Para evitar una de las principales complicaciones que presenta este método, el edema posreperfusión, se intenta limitar la cantidad de segmentos abiertos en cada sesión de tratamiento (según la mayoría de los centros expertos, solo dos segmentos). Habitualmente los pacientes requieren de aproximadamente 4 sesiones para lograr el objetivo (generalmente lograr una PAPm $\leq 30 \mathrm{mmHg}$ ), con una media de 3 vasos dilatados por sesión $n^{50}$.

Los trabajos publicados a la fecha coinciden en la mejoría hemodinámica y de CF de los pacientes sometidos a ABAP, los cuales debían de ser clasificados como inoperables por un grupo experimentado de cirujanos (dentro de los criterios se encontraban edad avanzada, comorbilidades y enfermedad distal).

En el año 2001 Feinstein et al. demostraron una mejoría significativa de la PAPm en promedio de $42 \pm 12 \mathrm{mmHg}$ a $33 \pm 10 \mathrm{mmHg}$ a los 6 meses de tratamiento. A su vez también mejoró de manera significativa la CF media de los pacientes (según la NYHA, de CF 3,3 a 1,8, en promedio) y la DR6 (de 209 a 497 yardas). En el seguimiento a 36 meses los parámetros se mantuvieron sin cambios significativos. Como 
Tabla 2. Resultados de las angioplastias con balón a arterias pulmonares realizadas en centros expertos.

\begin{tabular}{|c|c|c|c|c|c|c|}
\hline & $\begin{array}{c}\text { Número de } \\
\text { procedimientos }\end{array}$ & $\begin{array}{l}\text { RVP media } \\
\text { previo a ABAP* }\end{array}$ & $\begin{array}{c}\text { RVP media } \\
\text { posterior a } A B A P *\end{array}$ & $\begin{array}{c}\% \text { de disminución } \\
\text { de RVP }\end{array}$ & $\begin{array}{l}\text { Mortalidad peri- } \\
\text { procedimiento }\end{array}$ & Supervivencia \\
\hline Kataoka et al. & 29 & 472 & 280 & $59 \%$ & $3,4 \%$ & No disponible \\
\hline Mizoguchi et al. & 68 & 942 & 327 & $65 \%$ & $1,5 \%$ & $97 \%$ luego de una media de 2,2 años \\
\hline Sugimura et al. & 12 & 627 & 310 & $54 \%$ & $0 \%$ & $100 \%$ al año \\
\hline Fukui et al. & 20 & 889 & 490 & $45 \%$ & $0 \%$ & No disponible \\
\hline Andreassen et al. & 20 & 704 & 472 & $33 \%$ & $10 \%$ & $85 \%$ luego de una media de 4 años \\
\hline Taniguchi et al. & 29 & 763 & 284 & $63 \%$ & $3,4 \%$ & No disponible \\
\hline
\end{tabular}

*Medida en dyn.s.cm ${ }^{-5}$. ABAP: angioplastia con balón a las arterias pulmonares. RVP: resistencia vascular pulmonar.

ya se mencionó anteriormente, este estudio no estuvo exento de complicaciones: de 18 pacientes, 11 desarrollaron edema posreperfusión, resultando en la necesidad de asistencia respiratoria mecánica en 3 casos, y uno falleció ${ }^{47}$.

En el año 2012 Kataoka et al. publicaron un estudio de 29 pacientes en un centro en Japón. La ABAP demostró un descenso del 30\% en la PAPm que fue significativa en el seguimiento a 6 meses $(45,3 \pm 8$ a $31,8 \pm 10 \mathrm{mmHg}$ ) y de la RVP de $5,9 \pm 4,9$ a $3,5 \pm 1,7$ Unidades Wood $(p<0,001)$. A su vez se observó una mejoría en los niveles del péptido natriurético tipo B (BNP) $(306 \pm 271$ vs. $98 \pm 197 \mathrm{pg} / \mathrm{ml})$ y en la CF según NYHA $(p<0,01)^{52}$

De los 29 pacientes, uno falleció por una complicación del procedimiento (perforación del vaso por catéter guía). El 68\% desarrolló algún tipo de edema posreperfusión, aunque solo un paciente requirió asistencia respiratoria mecánica y ECMO. La mortalidad a 6 meses fue del 3,4\% $\%^{52}$

En el mismo año, Mizoguchi et al. presentaron un trabajo realizado en un centro de Japón con 68 pacientes. El seguimiento promedio fue de 2,2 años. Se observó una mejoría significativa en los parámetros hemodinámicos (PAPm de 45,4 $\pm 9,6$ a 24 $\pm 6,4$ mmHg, $p<0,001 ;$ RVP de 942 \pm 367 a

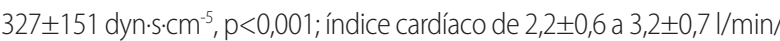
$\mathrm{m}^{2}, \mathrm{p}<0,001$ ), en los niveles de BNP (de 330 a $35 \mathrm{pg} / \mathrm{ml}, \mathrm{p}<0,001$ ), en la CF del paciente según la World Health Organization (WHO) y en la DR6 (de $296 \mathrm{~m}$ a 368m, p<0,001). Solo un paciente falleció durante el procedimiento, y en el seguimiento a 2,2 años promedio solo se registró una muerte de causa no cardiovascular ${ }^{50}$. Se resumen los resultados de los principales estudios con ABAP en la Tabla $\mathbf{2}^{49,50,52}$

En el año 2014, Inami et al. presentaron un estudio retrospectivo no aleatorizado con los datos obtenidos de tres instituciones de Japón, en el que se incluyó a 129 pacientes con HPTC. El criterio de selección de qué paciente iba a cada intervención fue más flexible que en los estudios antes mencionados. Fueron asignados a TEAP aquellos que presentaban las principales lesiones en localización proximal y no contaban con contraindicaciones para la cirugía. En aquellos con enfermedad a predominio distal o con discrepancia entre las lesiones angiográficas y el valor de presión pulmonar, se los sometía a ABAP. Asimismo, también se les realizaba angioplastia a aquellos que rechazaban la cirugía y a aquellos en que el equipo, por edad avanzada o comorbilidades, decidiese que se beneficiarían más con ese método. Por lo que 68 pacientes se sometieron ABAP y solo 39 TEAP. A los sometidos a estos dos procedimientos se los catalogó como grupo intervención. Además, existió otro grupo con 29 sujetos que no recibió ni ABAP ni TEAP, ya sea por negativa del mismo o por consideración del equipo tratante.
Estos recibieron solo tratamiento médico, siendo en general eran menos graves, la mayoría en CF II (a diferencia del grupo intervención con (F más avanzada), con mayor DR6, y con mejores parámetros hemodinámicos ${ }^{53}$.

En un seguimiento promedio a 49 meses se demostró que la supervivencia del grupo intervención era significativamente superior a la de grupo tratamiento médico ( $98 \%$ vs. $64 \% ; p<0,0001)^{53}$

Los pacientes sometidos a TEAP con respecto a los del grupo presentaban mayor compromiso hemodinámico (mayor PAPm y RVP, más concentración de BNP, y menor índice cardíaco) y funcional (DR6 de 283 vs. 342 m), pero eran más jóvenes (53 vs. 62 años) ${ }^{53}$

La mortalidad perioperatoria del grupo ABAP fue del 1,47\% (1 muerte de los 68 pacientes) contra 2,6\% del grupo TEAP (1 muerte de los 39 pacientes) $)^{53}$

Los parámetros hemodinámicos y la sintomatología (medida como (F según NYHA y DR6) en los dos grupos de intervención presentaron mejoría significativa con respecto al basal, sin diferencia significativa entre los dos grupos ${ }^{53}$ (Figuras 5 y $\mathbf{6}$ ).

En el seguimiento medio a 33 meses la mortalidad en el grupo ABAP fue del 1,5\% (1 muerte de los 68 pacientes sometidos, posprocedimiento) y en el grupo TEAP del 5,1\% (1 muerte posprocedimiento y otra por falla ventricular derecha en contexto de HTP residual). La necesidad de hospitalización fue del 1,5\% en el grupo ABAP contra un 10,3\% en el grupo TEAP ${ }^{53}$.

Este último estudio presenta importantes limitaciones entre las que se destacan el bajo número de pacientes y el no ser un estudio aleatorizado, por lo que las poblaciones sometidas a los distintos tratamientos son claramente distintas, presentando enfermedad más severa aquellos sometidos a cirugía.

Las complicaciones continúan siendo una de las limitaciones más importantes al mayor uso de este procedimiento. El edema posreperfusión es una complicación muy frecuente, con una incidencia aproximada del $53-60 \%$, aunque es severo solo en menos del 10\% de los casos. Factores de riesgo para su desarrollo son la presión arterial pulmonar elevada, el índice cardíaco bajo y la elevación del BNP. La primera sesión del procedimiento y el número de segmentos involucrados también se encuentran asociados ${ }^{48}$.

Para prevenir esta complicación se recomienda limitar el número de segmentos involucrados en cada sesión de angioplastia. Se validó un score, denominado PEPSI (Pulmonary Edema Predictive Score Index), como predictor del desarrollo del edema posreperfusión. Este resulta del producto entre el tipo de flujo pulmonar (del 0 al 3, de más a menos grave) y la RVP basal del paciente. A mayor valor, más probabilidad de presentar edema posreperfusión ${ }^{54}$. 

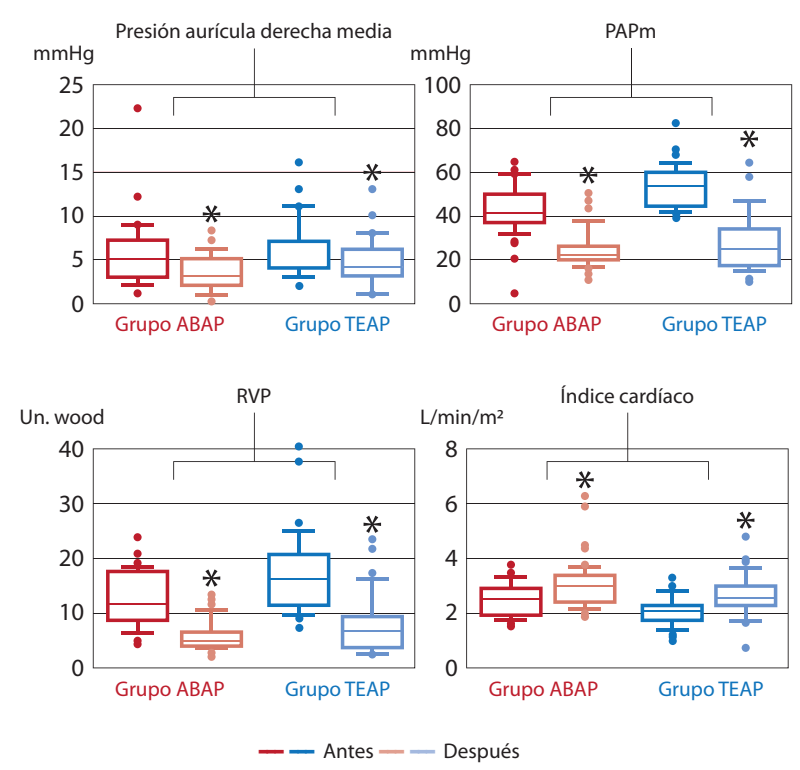

Figura 5. Cambios hemodinámicos en pacientes tratados con angioplastia con balón a las arterias pulmonares y en pacientes tratados con endarterectomía pulmonar. En el trabajo publicado por Inami et al. se observó una mejoría significativa de los parámetros hemodinámicos pre y post tratamiento, tanto en el grupo ABAP como en el grupo TEAP $(*)$. No se observó diferencia significativa entre los resultados de ambos tratamientos (). ABAP: angioplastic con balón a las arterias pulmonares. TEAP: tromboendarterectomía pulmonar. RVP: resistencia vascular pulmonar media. PAPm: presión arterial pulmonar media. Modificado de Inam T, Kataoka M, Ando M, Fukuda K, Yoshino H, Satoh T. A new era of therapeutic strategies for chronic thromboembolic pulmonary hypertension by two different interventional therapies; pulmonary endarterectomy and percutaneous transluminal pulmonary angioplasty. Plos One 2014;9(4):e94587.

Otra de las complicaciones más graves es la perforación de las ramas de la arteria pulmonar. Su incidencia es del 0 al 7\% y se encuentra asociado a elevadas tasas de mortalidad. Para evitarlo se debe controlar el correcto posicionamiento del catéter y el tamaño del balón debe ser proporcional al vaso objetivo ${ }^{48}$.

Como se mencionó previamente, la mortalidad asociada al procedimiento es menor al 5\% según las distintas series analizadas (Tabla 2). Según la última guía europea de hipertensión pulmonar publicada en el año 2015, la ABAP puede ser considerada en pacientes que son técnicamente inoperables o que presenten un desfavorable cociente riesgo/beneficio para la TEAP, en centros con experiencia en este procedimiento (grado de recomendación IIb, nivel de evidencia ()².

El abordaje del paciente debe ser en un centro experto en la patología, de forma interdiscipinaria. Existe un tratamiento establecido de la patología, la TEAP, por lo que la decisión de no operar a un paciente debe ser, al momento, muy bien fundamentada. Es por este motivo que algunos autores incluso recomiendan la evaluación por 2 equipos quirúrgicos distintos ${ }^{30}$.

La guía de la Sociedad Argentina de Cardiología publicada en el año 2011 no menciona la posibilidad de este tratamiento ${ }^{55}$.

\section{TRATAMIENTO FARMACOLÓGICO DIRIGIDO}

En la bibliografía se utiliza este término para referirse a los fármacos que tienen acción sobre la vasculatura pulmonar: inhibidores de la enzima fosfodiesterasa 5 (FDE-5) (p. ej., sildenafil), antagonistas de los receptores de endotelina 1 (p. ej., bosentán), análogos de la prosta-

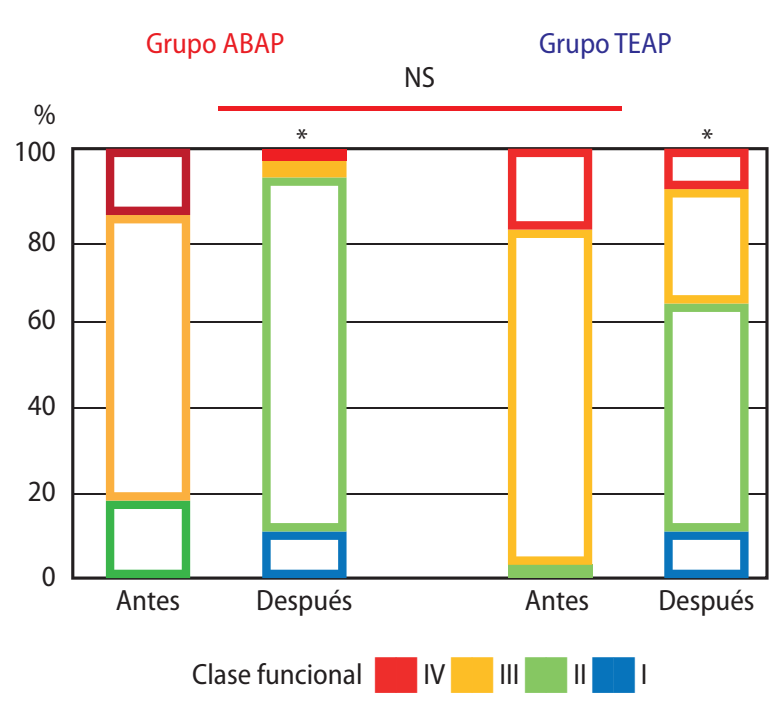

Figura 6. Mejoría de la clase funcional de los pacientes sometidos a angioplastia a las arterias pulmonares y tromboendarterectomía pulmonar. En el trabajo publicado por Inami et al. se observó una mejoría significativa de la CF de los pacientes pre-y postratamiento, tanto en el grupo ABAP como en el grupo TEAP $(*)$. Aproximadamente el $80 \%$ de los pacientes en el grupo ABAP y más del $90 \%$ en el grupo TEAP se encontraban con una clase funcional IIIIIV. Luego del tratamiento menos del $5 \%$ de los pacientes sometidos a ABAP y aproximadamente el $30 \%$ de los del grupo TEAP continuaron presentando disnea CF IIIIIV. No se observó diferencia significativa entre los resultados de am bos tratamientos. ABAP: angioplastia con balón a las arterias pulmonares. TEAP: tromboendarterectomía pulmonar. RVP: resistencia vascular pulmonar media. PAPm: presión arterial pulmonar media. NS: no significativo. Modificado de Inami T, Kataoka M, Ando M, Fukuda K, Yoshino H, Satoh T. A new era of therapeutic strategies for chronic thromboembolic pulmonary hypertension by two different interventional therapies; pulmonary endarterectomy and percutaneous transluminal pulmonary angioplasty. Plos One 2014;9(4):e94587.

ciclina e inhibidores de la guanilato ciclasa soluble (p. ej., riociguat) (Figura 5). El fundamento de su extendida utilización en el tratamiento de la HPTC se basa en la similitud fisiológica e histopatológica entre el compromiso de la vasculatura pulmonar periférica de la HAP primaria y la HPTC. Según el último registro europeo de HPTC, el $61 \%$ de los pacientes no operables recibe algún tipo de tratamiento farmacológico dirigido: el $17 \%$ se encontraba con sildenafil, el $24 \%$ con antagonistas de la endotelina 1, el 18\% con ambas drogas y solo el $2 \%$ con derivados de la prostaciclina. Es importante aclarar que, a la fecha de publicación del registro, todavía no había sido aprobado el riociguat para el tratamiento de la $\mathrm{HPTC}^{6}$. A pesar de su uso extendido, la evidencia para el uso de estos fármacos en esta patología es limitada.

El sildenafil actúa inhibiendo a la enzima FDE-5, esta se encarga de degradar el GMPc (guanosín monofosfato cíclico) a GMP (guanosín monofosfato). El aumento de los niveles de GMPc produce vasodilatación y disminución del remodelado vascular pulmonar, a través de la vía del óxido nítrico (ON). A pesar de su demostrada utilidad en HAP primaria, en HPTC ha sido estudiada solo en un pequeño ensayo clínico aleatorizado contra placebo en 19 pacientes con HPTC inoperable. Si bien hubo una mejoría en los parámetros hemodinámicos y en la CF de los pacientes, no hubo diferencias significativas en el punto final primario del estudio que era la DR6 ${ }^{56}$. 
El bosentán es un antagonista de los receptores de la endotelina-1 que, por lo tanto, inhibiría la vasoconstricción, la activación plaquetaria y el remodelado vascular. Esta droga encabezó el primer ensayo clínico de mayores dimensiones en pacientes con HPTC, el estudio BENEFiT ${ }^{57}$, el cual aleatorizó a 157 pacientes con HPTC inoperable o con HTP residual/persistente post-TEAP a recibir bosentán o placebo, con seguimiento por 16 semanas. Los puntos finales primarios del estudio fueron la DR6 y la disminución de la RVP. Si bien hubo mejoría significativa en la disminución de la RVP (un 24\%), no hubo diferencias significativas en la DR6.

El riociguat es un fármaco que actúa estimulando la actividad de la guanilato ciclasa soluble, por lo tanto aumenta los niveles de GMPc, generando un aumento de la vasodilatación y un efecto antiproliferativo y antifibrótico, mediante la vía del ON.

El estudio CHEST-1 fue un ensayo clínico randomizado en fase 3, en el cual se asignó a 261 pacientes con HPTC inoperable o con HTP residual/persistente post-TEAP a recibir riociguat o placebo en una relación 2:1. El punto final primario fue la DR6. En el seguimiento a 16 semanas, el fármaco aumentó significativamente la DR6 (el grupo riociguat aumentó una media de 39 m, en cambio el grupo placebo tuvo caída con respecto al basal de 6 m). También mejoró de forma estadísticamente significativa los parámetros hemodinámicos (la RVP disminuyó una media de 293 dyn.s. $\mathrm{cm}^{-5}$ en el grupo riociguat contra un aumento de 23 dyn.s.cm en el grupo placebo), el valor del BNP y la CF según $\mathrm{WHO}^{58,59}$.

Los 237 pacientes que terminaron dicho estudio fueron incorporados al estudio CHEST-2, un estudio en fase IV, abierto, en el que se evaluó la tolerabilidad y el perfil de efectos adversos a largo plazo. Además, se observó que los resultados obtenidos en el CHEST-1 fueron mantenidos tras un año de tratamiento. Los efectos adversos más importantes reportados fueron: nasofaringitis (24\%), mareos (19\%), edema periférico (15\%), disnea y tos (15\%), hipotensión arterial (10\%) y hemoptisis $(5 \%)^{60}$.

El riociguat, debido a los resultados positivos que presentó en estos estudios, es la única droga aprobada en el mercado para el tratamiento de la HPTC en pacientes inoperables, así como en aquellos con HTP residual/persistente luego de la TEAP. En este grupo de pacientes, según la guía europea de HTP, su uso es una indicación de grado I, nivel de evidencia $B^{2}$.

El resto del tratamiento farmacológico dirigido (antagonistas de la endotelina, inhibidores de la FDE-5, prostanoides) no ha sido aprobado para su uso en HPTC, aunque la guía europea habilita su uso con un grado de recomendación IIB, nivel de evidencia B².

Es importante recalcar que los grupos de pacientes que se benefician con el tratamiento farmacológico dirigido son aquellos que han sido correctamente evaluados y se han considerado inoperables y los que luego del gold standard de tratamiento para esta patología, la TEAP, continúan con presiones pulmonares elevadas sintomáticas.

\section{DISCUSIÓN}

La HPTC es una enfermedad infrecuente que acarrea elevada morbimortalidad. Probablemente su prevalencia sea mayor a la que constatada en los registros, por lo que es una enfermedad subdiagnosti- cada. Probablemente el factor que más influencia tenga en su subdiagnóstico es el desconocimiento de la comunidad médica sobre esta patología y el bajo índice de sospecha. Según el último registro europeo ${ }^{6}$, el tiempo entre el inicio de la sintomatología y el diagnóstico es de 15 meses. Otro elemento importante es la diferenciación de la HPTC con otros grupos de HTP, sobre todo la HAP primaria. La centollografía ventilación/perfusión, a pesar de ser un método accesible y con una sensibilidad cercana al 100\% para el diagnóstico de esta patología, continúa siendo poco utilizada. Por este motivo, un importante porcentaje de pacientes con HPTC es catalogado como HAP primaria.

El problema de subdiagnosticar la HPTC radica en que esta presenta un tratamiento de elección establecido, la TEAP, con excelentes resultados y potencial curabilidad. Sin este tratamiento, el pronóstico de la enfermedad es ominoso $0^{1,2}$.

La TEAP, a su vez, es una intervención quirúrgica compleja, por lo que requiere equipos de trabajo con amplia experiencia en su manejo. Pocos son los centros en el mundo que la realizan a gran escala. Existe una relación inversa entre la experiencia del centro y la mortalidad intrahospitalaria, por lo que los pacientes que deban ser intervenidos quirúrgicamente deben ser derivados a centros con mayor experiencia. La mortalidad intrahospitalaria según el último registro europeo es de menos del 5\%, similar a la del reemplazo valvular mitral $(6 \%)^{6}$.

A su vez, el correcto diagnóstico no asegura el correcto tratamiento. Esto se debe principalmente a una falta de criterios unificados en la evaluación preoperatoria del paciente, por lo tanto, la decisión de la posibilidad quirúrgica o no depende del equipo quirúrgico tratante. Un paciente podría ser no operable para un equipo quirúrgico y serlo para otro. Un ejemplo de esto puede observarse en el estudio BENEFiT con bosentán ${ }^{57}$, en el cual la inoperabilidad de los pacientes se decidía en un primer paso por un médico y un cirujano expertos, y en un segundo paso por 2 neumólogos y otros 2 cirujanos expertos. Se pudo apreciar que 11 de los 157 pacientes del estudio, que fueron catalogados como inoperables por el primer equipo, en la reevaluación por un segundo equipo fueron excluidos del estudio por considerárselos operables. Por estos motivos, es necesario un consenso en donde se plasmen los criterios de no operabilidad, para que la decisión no recaiga en la subjetividad de un único equipo. Mientras tanto, es necesario que los pacientes sean correctamente evaluados, si es posible, por más de un equipo experto.

A pesar de una correcta evaluación, continuaría habiendo un importante número de pacientes con enfermedad inoperable, ya sea por predominancia de enfermedad microvascular o por un cociente riesgo/beneficio desfavorable. Hasta hace unos pocos años no existía terapéutica para ofrecer a este tipo de pacientes.

En los últimos años, ha habido importantes avances para el tratamiento de este subgrupo de pacientes. La ABAP es una intervención prometedora. Su uso todavía se encuentra limitado a unos pocos centros que la realizan, la mayoría en Japón. Si bien los resultados que se obtuvieron con este procedimiento son alentadores, no está exento de riesgos (sobre todo, edema posreperfusión y perforación por catéter), no se conoce todavía la evolución de los pacien- 
tes a largo plazo y en ningún trabajo realizado el punto final primario fue mortalidad. Un potencial riesgo de este nuevo procedimiento es que se amplíe la indicación no solo a pacientes inoperables, sino también a aquellos que puedan ser candidatos a cirugía. En este caso, es necesario realizar ensayos clínicos randomizados que comparen ambas estrategias.

Para los pacientes inoperables o con HTP persistente luego de la TEAP, se encuentra como alternativa terapéutica el tratamiento médico dirigido. El único fármaco aprobado para su uso es el riociguat ${ }^{58,59}$. El punto final primario evaluado fue la DR6, un punto bastante blando. No ha sido probado en pacientes con enfermedad operable, y de hecho no se encuentra recomendado en estos casos, ya que se ha observado en registros que aumenta el tiempo hasta la cirugía.

Si bien el escenario es prometedor, no debe llevarnos a tomar decisiones erradas. La HPTC tiene un gold standard de tratamiento, la TEAP, por lo que todo paciente a priori debe recibirlo². Si bien las nuevas terapéuticas son alentadoras, al momento no la reemplazan. Todavía hay mucho que investigar.

\section{BIBLIOGRAFÍA}

1. Hoeper MM, Madani MM, Nakanishi N, Meyer B, Cebotari S, Rubin U. Chronic thromboembolic pulmonary hypertension. Lancet Respir Med 2014;2(7):573-82.

2. Galiè N, Humbert M, Vachiery JL, Gibbs S, Lang I, Torbicki A, et al. 2015 ESC/ERS Guidelines for the diagnosis and treatment of pulmonary hypertension. Eur Heart J 2016;37(1):67-119

3. Lang IM. Chronic thromboembolic pulmonary hypertension--not so rare after all. N Engl J Med 2004;350(22):2236-8.

4. Pengo V, Lensing AW, Prins MH, Marchiori A, Davidson BL, Tiozzo F, et al. Incidence of chronic thromboembolic pulmonary hypertension after pulmonary embolism. N Engl J Med 2004;350(22):2257-64.

5. Pepke-Zaba J, Delcroix M, Lang I, Mayer E, Jansa P, Ambroz D, et al. Chronic thromboembolic pulmonary hypertension (CTEPH): results from an international prospective registry. Circulation 2011;124(18):1973-81.

6. Delcroix M, Lang I, Pepke-Zaba J, Jansa P, D'Armini AM, Snijder R, et al. Longterm outcome of patients with chronic thromboembolic pulmonary hypertension: results from an international prospective registry. Circulation 2016;133(9):859-71.

7. O'Connell C, Montani D, Savale L, Sitbon O, Parent F, Seferian A, et al. Chronic thromboembolic pulmonary hypertension. Presse Med 2015;44(12):e409-e416.

8. Escribano-Subias P, Blanco I, López-Meseguer CJ, Lopez-Guarch CJ, Roman A, Morales $P$, et al. Survival in pulmonary hypertension in Spain: insights from the Spanish registry. Eur Respir J 2012;40(3):596-603.

9. Coronel ML, Perna ER, Echazarreta D, Einer Allende G, Cimbraro Canella JP, Pereiro $S$, et al. Treatment of pulmonary arterial hypertension according with functional class in the Argentinean HINPULSAR Registry. Eur J Heart Fail 2012;11(s1):555.

10. Echazarreta D, Perna E, Coronel ML, Diez M, Lescano A, Atamañuk N, et al. I Registro Colaborativo de Hipertensión Pulmonar en Argentina (RECOPILAR). Rev Fed Arg Cardiol 2014:43(3):146-149.

11. McLaughlin WV, Langer A, Tan M, Clements PJ, Oudiz RJ, Tapson VF, O et al. Contemporary trends in the diagnosis and management of pulmonary arterial hypertension: an initiative to close the care gap. Chest 2013;143(2):324-332.

12. Kim NH, Delcroix M, Jenkins DP, Channick R, Dartevelle P, Jansa P, et al. Chronic thromboembolic pulmonary hypertension. J Am Coll Cardiol 2013;62(25):92-9.

13. Kim NH. Group 4 Pulmonary Hypertension: Chronic Thromboembolic Pulmonary Hypertension: Epidemiology, Pathophysiology, and Treatment. Cardiol Clin 2016;34(3):435-41.

14. Hirsh J, Hoak J. Management of deep vein thrombosis and pulmonary embolism. A statement for healthcare professionals from the council on thrombosis (in consultation with the council on cardiovascular radiology), American Heart Association. Circulation 1996;93(12):2212-45.

\section{CONCLUSIÓN}

La HPTCa es una complicación infrecuente del TEP que, en ausencia de tratamiento, se encuentra asociada a una elevada morbimortalidad. Su clínica inespecífica y el desconocimiento de su manejo diagnóstico por gran parte de la comunidad médica hace que sea una enfermedad subdiagnosticada.

La importancia de su diagnóstico correcto radica en que esta forma de HTP tiene un tratamiento específico, potencialmente curativo, la TEAP.

Dado que aproximadamente solo un 40\% de los pacientes son inoperables, resulta crítico el reconocimiento clínico y la familiarización con las nuevas estrategias terapéuticas mínimamente invasivas. La única droga aprobada en este escenario es el riociguat. La ABAP es una estrategia prometedora para pacientes seleccionados con enfermedad inoperable. Sin embargo, todavía se desconocen sus resultados a largo plazo por lo que su papel en el tratamiento de la HPTC descansa aún en una promesa, aunque alentadora.
15. Klok FA, van Kralingen KW, van Dijk AP, Heyning FH, Vliegen HW, Huisman MV Prospective cardiopulmonary screening program to detect chronic thromboembolic pulmonary hypertension in patients after acute pulmonary embolism. Haematologica 2010;95(6):970-5.

16. Barnes AR, Yater WM. Failure of the right ventricle due to an ancient thrombus of the pulmonary arteries. M Clin North America 1929;12:1603.

17. Toshner M, Pepke-Zaba J. Chronic thromboembolic pulmonary hypertension: time for research in pathophysiology to catch up with developments in treatment. F1000Prime Rep 2014;6:38.

18. Matthews DT, Hemnes AR. Current concepts in the pathogenesis of chronic thromboembolic pulmonary hypertension. Pulm Circ 2016;6(2):145-54.

19. Moser KM, Bloor CM. Pulmonary vascular lesions occurring in patients with chronic major vessel thromboembolic pulmonary hypertension. Chest 1993;103(3):685-92.

20. Winebright JW, Gerdes AJ, Nelp WB. Restoration of blood flow after pulmonary embolism. Arch Intern Med 1970;125(2):241-7.

21. Nijkeuter M, Hovens MM, Davidson BL, Huisman MV. Resolution of thromboemboli in patients with acute pulmonary embolism: a systematic review. Chest 2006;129(1):192-7.

22. Ribeiro A, Lindmarker P, Johnsson H, Juhlin-Dannfelt A, Jorfeldt L. Pulmonary embolism: one-year follow-up with echocardiography doppler and five-year survival analysis. Circulation 1999:99(10):1325-30.

23. Morris, TA. Why acute pulmonary embolism becomes chronic thromboembolic pulmonary hypertension: clinical and genetic insights. Curr Opin Pulm Med 2013;19(5):422-9.

24. Kim NH, Lang IM. Risk factors for chronic thromboembolic pulmonary hypertension. Eur Respir Rev 2012;21(123):27-31.

25. Lisman T, de Groot PG, Meijers JC, Rosendaal FR. Reduced plasma fibrinolytic potential is a risk factor for venous thrombosis. Blood 2005;105(3):1102-5.

26. Moser KM, Cantor JP, Olman M, Villespin I, Graif JL, Konopka R, et al. Chronic pulmonary thromboembolism in dogs treated with tranexamic acid. Circulation 1991;83(4):1371-9.

27. Lang IM, Marsh JJ, Olman MA, Moser KM, Loskutoff DJ, Schleef RR. Expression of type 1 plasminogen activator inhibitor in chronic pulmonary thromboemboli. Circulation 1994;89(6):2715-21.

28. Morris TA, Marsh JJ, Chiles PG, Auger WR, Fedullo PF, Woods VL. Fibrin derived from patients with chronic thromboembolic pulmonary hypertension is resistant to lysis. Am J Respir Crit Care Med 2006;173(11):1270-1275.

29. Quarck R, Wynants M, Verbeken , , flammation and impaired angiogenesis to the pathobiology of chronic thromboembolic pulmonary hypertension. Eur Respir J 2015;46(2):431-43. 
30. Lang IM, Madani M. Update on chronic thromboembolic pulmonary hypertension. Circulation 2014;130(6):508-518

31. Lang IM, Simonneau G, Pepke-Zaba JW, Mayer E, Ambrož D, Blanco l et al. Factors associated with diagnosis and operability of chronic thromboembolic pulmonary hypertension. A case-control study. Thromb Haemost 2013;110(1):83-91.

32. Tunariu N, Gibbs SJ, Win Z, Gin-Sing W, Graham A, Gishen P et al. Ventilation-perfusion scintigraphy is more sensitive than multidetector CTPA in detecting chronic thromboembolic pulmonary disease as a treatable cause of pulmonary hypertension. J Nucl Med 2007;48(5):680-4.

33. MCLaughlin WV Archer SL, Badesch DB, Barst RJ, Farber HW, Lindner JR et al. ACCF/ AHA 2009 expert consensus document on pulmonary hypertension a report of the American College of Cardiology Foundation Task Force on expert consensus documents and the American Heart Association developed in collaboration with the American College of Chest Physicians, American Thoracic Society, Inc.; and the Pulmonary Hypertension Association. J Am Coll Cardiol 2009;53(17):1573-619.

34. Riedel M, Stanek V, Widimsky J, Prerovsky I. Longterm follow-up of patients with pulmonary thromboembolism. Late prognosis and evolution of hemodynamic and respiratory data. Chest 1982;81(2):151-8.

35. Corsico AG, D'Armini AM, Cerveri I, Klersy C, Ansaldo E, Niniano R, et al. Longterm outcome after pulmonary endarterectomy. Am J Respir Crit Care Med 2008; 178(4):419-24.

36. Jenkins D. Pulmonary endarterectomy: the potentially curative treatment for patients with chronic thromboembolic pulmonary hypertension. Eur Respir Rev 2015;24(136): 263-71

37. Madani MM, Auger WR, Pretorius V, Sakakibara N, Keer KM, Kim NH et al. Pulmonary endarterectomy: recent changes in a single institution's experience of more than 2,700 patients. Ann Thorac Surg 2012;94(1):97-103.

38. Berman M, Hardman G, Sharples L, Pepke-Zaba J, Sheares K, Tsui S, et al. Pulmonary endarterectomy: outcomes in patients aged > 70. Eur J Cardiothorac Surg 2012:41(6):154-60

39. Banks DA, Pretorius GV, Kerr KM, Manecke GR. Pulmonary endarterectomy: Part II. Operation, anesthetic management, and postoperative care. Semin Cardiothorac Vasc Anesth 2014;18(4):331-40

40. Jamieson SW, Kapelanski DP, Sakakibara N, Manecke GR, Thistlethwaite PA, Keer KM et al. Pulmonary endarterectomy: experience and lessons learned in 1,500 cases. Ann Thorac Surg 2003;76(5):1457-62.

41. Favaloro RR, Peradejordi MA, Gómez CB, Santos M, Caneva JO, Klein F, et al. Tromboendarterectomía pulmonar: tratamiento de elección para la hipertensión pulmonar tromboembólica crónica. Rev Am Med Resp 2011;11(2):74-83.

42. Vuylsteke A, Sharples L, Charman G, Kneeshaw J, Tsui S, Dunning J, et al. Circulatory arrest versus cerebral perfusion during pulmonary endarterectomy surgery (PEACOG): a randomised controlled trial. Lancet 2011;378(9800):1379-87.

43. Skoro-Sajer N, Hack N, Sadushi-Koliçi R, Bonderman D, Jakowitsch J, Klepetko W, et al. Pulmonary vascular reactivity and prognosis in patients with chronic thromboembolic pulmonary hypertension: a pilot study. Circulation 2009;119(2):298-305.

44. Shimura N, Kataoka M, Inami T, Yanagisawa R, Ishiguro H, Kawakami T et al. Additional percutaneous transluminal pulmonary angioplasty for residual or recurrent pulmonary hypertension after pulmonary endarterectomy. Int I Cardiol 2015; 183:138-42
45. Lock JE, Castaneda-Zuniga WR. Fuhrman BP, Bass JL. Balloon dilation angioplas ty of hypoplastic and stenotic pulmonary arteries. Circulation 1983;67(5):962-7.

46. Voorburg JA, Cats VM, Buis B, Bruschke AV. Balloon angioplasty in the treat ment of pulmonary hypertension caused by pulmonary embolism. Chest 1988:94(6):1249-53.

47. Feinstein JA, Goldhaber SZ, Lock JE, Ferndandes SM, Landzberg MJ. Balloon pulmonary angioplasty for treatment of chronic thromboembolic pulmonary hypertension. Circulation 2001;103(1):10-13.

48. Ogo T. Balloon pulmonary angioplasty for inoperable chronic thromboembolic pulmonary hypertension. Curr Opin Pulm Med 2015;21(5):425-31.

49. Andreassen AK, Ragnarsson A, Gude E, Geiran O, Andersen R. Balloon pulmonary angioplasty in patients with inoperable chronic thromboembolic pulmonary hypertension. Heart 2013:99(19):1415-20.

50. Mizoguchi H, Ogawa A, Munemasa M, Mikouchi H, Ito H, Matsubara H. Refined balloon pulmonary angioplasty for inoperable patients with chronic thromboem bolic pulmonary hypertension. Circ Cardiovasc Interv 2012:5(6):748-55.

51. Tatebe S, Sugimura K, Aoki T, Miura M, Nochioka K, Yaoita N et al. Multiple beneficial effects of balloon pulmonary angioplasty in patients with chronic thrombo embolic pulmonary hypertension. Circ J 2016;80(4):980-8.

52. Kataoka M, Inami T, Hayashida K, Shimura N, Ishiguro H, Abe T et al. Percutaneous transluminal pulmonary angioplasty for the treatment of chronic thrombo embolic pulmonary hypertension. Circ Cardiovasc Interv 2012;5(6):756-62.

53. Inami T, Kataoka M, Ando M, Fukuda K, Yoshino H, Satoh T. A new era of therapeutic strategies for chronic thromboembolic pulmonary hypertension by two different interventional therapies; pulmonary endarterectomy and percutaneous transluminal pulmonary angioplasty. PloS One 2014;9(4):e94587.

54. Inami T, Kataoka M, Shimura N, Ishiguro H, Yanagisawa R, Taguchi Het al. Pulmonary edema predictive scoring index (PEPSI), a new index to predict risk of reperfusion pulmonary edema and improvement of hemodynamics in percutaneous transluminal pulmonary angioplasty. JACC Cardiovasc Interv 2013;6(7):725-36.

55. Vulcano N, Boughen R, Catoggio L, Comignani P, Diez M, Favaloro L et al. Consenso para el diagnóstico y tratamiento de la hipertensión arterial pulmonar. Sociedad Argentina de Cardiología (SAC), Asociación Argentina de Medicina Respiratoria (AAMR), Sociedad Argentina de Reumatología (SAR). Rev Argent Cardio 2011;79(2):1-24

56. Suntharalingam J, Treacy CM, Doughty NJ, Goldsmith K, Soon E, Toshner MR et al. Long-term use of sildenafil in inoperable chronic thromboembolic pulmonary hypertension. Chest 2008;134(2):229-236

57. Jaïs X, D'Armini AM, Jansa P, Torbicki A, Delcroix M, Ghofrani HA et al. Bosentan for treatment of inoperable chronic thromboembolic pulmonary hypertension: BENEFiT (Bosentan Effects in iNopErable Forms of chronlc Thromboembolic pulmonary hypertension), a randomized, placebo-controlled trial. J Am Coll Cardiol 2008;52(25):2127-34

58. Ghofrani HA, D'Armini AM, Grimminger F, Hoeper MM, Jansa P, Kim NH et al. Riociguat for the treatment of chronic thromboembolic pulmonary hypertension. $N$ Engl J Med 2013;369(4):319-29.

59. Simonneau G, D'Armini AM, Ghofrani HA, Grimminger F, Hoeper MM, Jansa P et al. Riociguat for the treatment of chronic thromboembolic pulmonary hypertension: a long-term extension study (CHEST-2). Eur Respir J 2015:45(5):1293-302. 


\title{
ESTRATEGIAS INTRAHOSPITALARIAS DE MEJORA DEL TIEMPO PUERTA-BALÓN Y EVOLUCIÓN CLÍNICA EN PACIENTES CON INFARTO AGUDO DE MIOCARDIO CON SUPRADESNIVEL DEL ST
}

\author{
INTRAHOSPITAL STRATEGIES FOR IMPROVING DOOR-TO-BALLOON \\ TIME AND CLINICAL EVOLUTION IN PATIENTS WITH ACUTE \\ MYOCARDIAL INFARCTION WITH ST-SEGMENT ELEVATION
}

\author{
ALAN SIGAL ${ }^{1}$, ANDREÍNA GIL RAMÍREZ' ${ }^{1}$ CRISTIAN GARMENDIA ${ }^{1}$, NOELIA ALBY ${ }^{1}$, LUCRECIA M. BURGOS², FERNANDO CURA ${ }^{3}$, \\ ALFONSINA CANDIELLO ${ }^{4}$
}

\begin{abstract}
RESUMEN
Introducción. El tratamiento exitoso del infarto con supradesnivel del ST (IAMCEST) es tiempo dependiente. Existe limitada información en nuestro país acerca de la efectividad de estrategias en la reducción de los tiempos de isquemia. E objetivo de este trabajo es analizar el impacto de la aplicación de estrategias intrahospitalarias de mejora (EIHM) en la reducción del tiempo puerta balón (TPB), y en la evolución clínica de pacientes con IAMCEST.

Métodos. Estudio observacional retrospectivo sobre una base de datos prospectiva desde el año 2014 hasta 2017. Se incluyeron de forma consecutiva pacientes con IAMCEST de $<12$ horas de evolución, que ingresaron para angioplastia primaria; $y$ se compararon según el período: pre- (2014-2015) o posimplementación de EIHM (2016-2017). Las ElHM evaluadas fueron: preactivación de hemodinamia y bypass de guardia. Como punto final primario se analizó el TPB. Como puntos secundarios se evaluó mortalidad a 180 días, reinfarto y nueva revascularización.

Resultados. Se analizaron 155 pacientes, 40,6\% dentro del grupo pre-EIHM y 59,4\% en el grupo post-EIHM. El 67\% llegó en ambulancia y el restante por sus propios medios. No existieron diferencias respecto al tratamiento médico instaurado (74\% recibió ticagrelor). Se observó una reducción del TPB en el grupo post-EIHM en comparación al preimplementación, de 42 min (rango intercuartílico $25-75$ [RIC]: 30-55,5) vs. 55 min (RIC: $32-77), p=0.014$, y en el tiempo total de isquemia, de 161,5 min (RIC: 120$239,5)$ vs. $197 \mathrm{~min}(145-277), p=0,02$. No se encontró diferencia significativa en mortalidad intrahospitalaria ( 3,1 vs. $2,1 \% ; p=0,7)$ ni en los eventos clínicos. El seguimiento se realizó en el 94,2\% de los pacientes, con una mediana de 7 meses (RIC: 1,23-15,7). A los 180 días no hubo diferencias en el punto final combinado (27,3 grupo pre- vs. $15,3 \%$ post-EIHM; log rank test $p=0,33$ ). En el modelo de regresión de Cox, la ElHM tuvo un hazard ratio de 0,69 (intervalo de confianza 95\%: 0,32-1,47 p=0,34).

Conclusión. Las EIHM implementadas lograron disminuir de forma significativa el TPB al igual que el tiempo total de isquemia, indicadores de la calidad del centro y del pronóstico intrahospitalario. Se observó una tendencia a la disminución del evento combinado en el seguimiento, aunque de forma no significativa.
\end{abstract}

Palabras clave: infarto del miocardio; infarto del miocardio con elevación del ST estrategias locales; mejoramiento de la calidad.

\section{ABSTRACT}

Introduction: The successful treatment of infarction with ST elevation (STEMI) is time dependent. There is limited information in our country about the effectiveness of strategies in reducing ischemia times. The aim of this work is to analyze the impact of the application of intrahospital strategies for improvement (IHSI) in the reduction of door-to-balloon time (DTB), and in the clinical evolution of patients with STEMI.

Methods: Retrospective observational study on a prospective database from 2014 to 2017. Patients with STEMI of $<12$ hours of evolution who were admitted for primary angioplasty were consecutively included; and they were compared according to the period: pre (2014-2015) or post implementation of IHSI (2016 2017). The evaluated IHSI were: preactivation of the hemodynamics lab and emergency bypass. The DTB was analyzed as the primary end point. As secondary points, 180 -day mortality, reinfarction and revascularization were evaluated. Results: 155 patients were analyzed, $40.6 \%$ in the pre-IHSI group and $59.4 \%$ in the post-IHSI group. $67 \%$ arrived by ambulance and the rest by their own means. There were no differences regarding the medical treatment established $(74 \%$ received ticagrelor). A reduction of DTB was observed in the post-IHSI group compared to the pre-implementation time; i.e. $42 \mathrm{~min}$ (PCTL 25-75: 30-55.5) vs. $55 \mathrm{~min}$ ( $P C T L$ 25-75: 32-77) $P=0.014$, and in the total ischemia time $161.5 \mathrm{~min}$ (PCTL 25 75 120-239.5) vs $197 \mathrm{~min}$ (145-277), $p=0.02$. No significant difference was found in in-hospital mortality (3.1 vs. $2.1 \%, p=0.7$ ) or in clinical events. Follow-up was performed in $94.2 \%$ of the patients, with a median of 7 months (PCTL 25-75: 1.23-15.7). At 180 days there were no differences in the composite end point (27.3 pre group vs. $15.3 \%$ post IHSI, log rank test $p=0.33$ ). In the Cox regression model, the IHSI had a hazard ratio of 0.69 ( $95 \%$ confidence interval $0.32-1.47 p=0.34$ ).

Conclusion: The implemented IHSI managed to significantly reduce the DTB, as well as the total time of ischemia, indicators of the quality of the center and the intrahospital prognosis. There was a tendency to decrease the composite event in the follow-up, although in a non-significant way.

Keywords: ST elevation; myocardial infarction; myocardial infarction; local strategies; quality Improvement.
1. Residente de Cardiología Clínica

2. Jefe de Residentes de Cardiología Clínica.

3. Jefe del Servicio de Cardiología Intervencionista y Terapéuticas Endovasculares.

4. Servicio de Cardiología Intervencionista y Terapéuticas Endovasculares. Instituto

Cardiovascular de Buenos Aires. Directora Iniciativa Stent - Save a Life. Argentina.

$\triangle$ Correspondencia: Dr. Alan Sigal. Blanco Encalada 1543, C1428DCO CABA, Rep. Argentina.alanrsigal@gmail.com.

Los autores declaran no tener conflictos de intereses.

Recibido: 14/10/2018| Aceptado: 01/11/2018

\section{INTRODUCCIÓN}

La enfermedad cardiovascular constituye la principal causa de muerte a nivel mundial, y en la Argentina no es una excepción, representando el 40\% ${ }^{1}$. La expresión más aguda de la cardiopatía isquémica es el infarto con elevación del segmento ST (IAMCEST).

La mortalidad en estos casos depende múltiples factores, pudiendo dividirse en los que involucran al paciente, como la edad, clasificación de Killip y Kimball y comorbilidades, y las que involucran a la atención médica recibida, principalmente el tiempo entre el inicio del dolor y la reperfusión?2.

El retraso del sistema, incluyendo la demora del paciente en consultar a servicios de emergencia, el tiempo en el traslado a centros con hemodinamia 
Tabla 1. Características basales. IMC: índice de masa corporal. ACV: accidente cerebrovascular. AHF: antecedentes heredofamiliares. ATC: angioplastía transcutánea. CRM: Cirugía de revascularización miocárdica. ElHM: estrategias intrahospitalarias de mejora. EPOC: enfermedad pulmonar obstructiva crónica. EVP: enfermedad vascular periférica. ERC: enfermedad renal crónica. HTA: hipertensión. IAM: infarto agudo de miocardio.

\begin{tabular}{|c|c|c|c|}
\hline & Pre-EIHM $(n=63)$ & Post-EIHM (n=92) & $\mathrm{p}$ \\
\hline Edad (media $\pm \mathrm{DE}$, años) & $63,4 \pm 10,9$ & $62,8 \pm 12$ & 0,73 \\
\hline Sexo masculino $(n, \%)$ & $49(77,8 \%)$ & $22(23,9 \%)$ & 0,8 \\
\hline IMC (kg/m2) & $27,1 \pm 3,5$ & $27,3 \pm 3,8$ & 0,7 \\
\hline HTA $(n, \%)$ & $40(63,5 \%)$ & $60(65,2 \%)$ & 0,82 \\
\hline Diabetes (n, \%) & $8(12,7 \%)$ & $14(15,2 \%)$ & 0,65 \\
\hline Dislipemia (n, \%) & $30(47,6 \%)$ & $62(67,4 \%)$ & 0,014 \\
\hline Tabaquismo (n, \%) & $27(42,9 \%)$ & $26(28,3 \%)$ & 0,06 \\
\hline $\operatorname{AHF}(n, \%)$ & $14(22,2 \%)$ & $17(18,5 \%)$ & 0,56 \\
\hline EPOC $(n, \%)$ & $0(0 \%)$ & $4(4,3 \%)$ & 0,094 \\
\hline ACV previo (n, \%) & $1(1,6 \%)$ & $0(0 \%)$ & 0,22 \\
\hline EVP previa(n, \%) & $3(4,8 \%)$ & $6(6,5 \%)$ & 0,64 \\
\hline $\mathrm{ERC}(\mathrm{n}, \%)$ & $0(0 \%)$ & $2(2,2 \%)$ & 0,23 \\
\hline IAM previo & $8(12,7 \%)$ & $16(17,4 \%)$ & 0,42 \\
\hline ATC previa & $9(14,3 \%)$ & $23(25 \%)$ & 0,1 \\
\hline CRM previa & $1(1,6 \%)$ & $4(4,3 \%)$ & 0,33 \\
\hline
\end{tabular}

y las demoras en derivación; es decir, todo el tiempo entre el inicio del dolor y la reperfusión adecuada, se asocia con mayor tamaño del infarto 3 y mayor mortalidad a corto y largo plazo ${ }^{24}$, siendo menor en los casos en los que se logra la reperfusión en menos de 60 minutos. Inclusive, se demostró que e tiempo entre la consulta y la activación del laboratorio de hemodinamia es un predictor independiente de mortalidad intrahospitalaria².

En este contexto, es fundamental diseñar e implementar estrategias a nivel institucional que logren disminuir los tiempos de cada centro, con el propósito de mejorar la sobrevida y evolución de los pacientes. Por lo tanto, el objetivo del presente trabajo fue analizar el impacto de la aplicación de estrategias intrahospitalarias de mejora (EIHM) en la reducción del tiempo puerta balón (TPB) y en la evolución clínica de pacientes con IAMCEST.

\section{MATERIALES Y MÉTODOS}

Se realizó un estudio observacional retrospectivo sobre una base de datos digitalizada cuya información fue recolectada en forma prospectiva desde el año 2014 hasta 2017 en el Instituto Cardiovascular de Buenos Aires (ICBA)

Se incluyeron pacientes mayores de 18 años con diagnóstico de IAMCEST de menos de 12 horas de evolución, que ingresaron traídos por ambulancia para angioplastia primaria (ATCP); y se compararon según el período: pre- (2014-2015) o posimplementación de EIHM (2016-2017).

Se definió IAMCEST acorde a la tercera definición universal de infarto y a las guías europeas de práctica clínica ${ }^{5,6}$. Se excluyeron pacientes que no cumplieran criterios de IAMCEST, con evolución mayor a 12 horas y aquellos que ingresaron derivados de otro centro.

En el año 2014 se implementaron dos ElHM: la preactivación de hemodinamia y el bypass de la guardia. Se estableció una red de comunicación entre servicios de emergencia médica que evaluaron pacientes en domicilio con dolor torácico y evidencia en electrocardiograma de supradesnivel del segmento ST y operadores entrenados en nuestro centro. Con el consenso diagnóstico de IAMCEST, se realizó la preactivación
Tabla 2. Tratamiento inicial. ElHM: estrategias intrahospitalarias de mejora.

$\begin{array}{lccc} & \text { Pre-EIHM }(n=63) & \text { Post-EIHM (n=92) } & p \\ \text { Prasugrel }(n, \%) & 1(1,6 \%) & 3(3,3 \%) & 0,51 \\ \text { Ticagrelor }(n, \%) & 49(77,8 \%) & 68(73,9 \%) & 0,58 \\ \text { Clopidogrel }(n, \%) & 10(15,9 \%) & 20(21,7 \%) & 0,36 \\ \text { Aspirina }(n, \%) & 60(95,2 \%) & 84(91,3 \%) & 0,34 \\ \text { Estatinas }(n, \%) & 54(85,7 \%) & 73(79,3 \%) & 0,22\end{array}$

Tabla 3. Resultados comparativos del punto final primario y del tiempo ECG-balón y total de isquemia. ECG: electrocardiograma. ElHM:estrategias intrahospitalarias de mejora.

\begin{tabular}{lccc} 
& Pre-EIHM $(\mathrm{n}=63)$ & Post-EIHM $(\mathrm{n}=92)$ & $\mathrm{p}$ \\
\hline $\begin{array}{l}\text { Tiempo total de isquemia } \\
\text { (mediana; RIC) }\end{array}$ & $197(145-277)$ & $161.5(120-239.5)$ & 0,02 \\
$\begin{array}{l}\text { Tiempo ECG-balón } \\
\text { (mediana; RIC) }\end{array}$ & $85(61-117)$ & $70(41-108,5)$ & 0.012 \\
$\begin{array}{l}\text { Tiempo puerta-balón } \\
\text { (mediana; RIC) }\end{array}$ & $55(32-77)$ & $42(30-55,5)$ & 0,014
\end{tabular}

de hemodinamia consistente en la llegada de médicos intervencionistas y preparación de la sala previo al arribo del paciente. Y posteriormente se implementó el bypass de la guardia, consistente en el ingreso directo del paciente a la sala sin previa evaluación por guardia de emergencias. El seguimiento fue realizado mediante controles ambulatorios y de forma telefónica a los 30,60 y 180 días del alta.

Como punto final primario se analizó el TPB. Como puntos secundarios se evaluó mortalidad a 180 días, reinfarto y nueva revascularización coronaria.

\section{ANÁLISIS ESTADÍSTICO}

Las variables categóricas se expresaron mediante números y porcentajes, y las continuas, según su distribución, como media o mediana, con su correspondiente desvío estándar o rango intercuartílico (RIC). Para evaluar la asociación entre las variables continuas se utilizó el test de U de Mann-Whitney o test de T, y para las categóricas se utilizó el test exacto de Fisher o Chi Cuadrado, según correspondiera. El análisis de sobrevida entre ambos subgrupos se construyó utilizando el método de Kaplan-Meier y se comparó mediante la prueba de log rank, y se realizó un modelo de regresión múltiple por método de Cox, expresado como hazard ratio (HR) y su intervalo de confianza de 95\% (IC95\%). Se incluyeron en el modelo las variables que presentaron una $p<0,05$ en el análisis univariado. Se consideró estadísticamente significativo un valor $p$ a dos colas menor o igual a 0,05. Para el análisis estadístico se utilizó el software SPSS 23.

\section{CONSIDERACIONES ÉTICAS}

El estudio fue conducido de acuerdo con las normas éticas nacionales (ley CABA 3301) y Ley nacional de investigación clínica en seres humanos, declaración de Helsinki y contó con la aprobación del Comité de Investigación y Ética de nuestra Institución.

\section{RESULTADOS}

Se incluyeron 155 pacientes, 63 en el primer grupo y 92 en el segundo. El promedio de edad entre los dos grupos fue de 63,1 años; $46 \%$ eran de sexo masculino; $64 \%$ hipertensos, $13,5 \%$ diabéticos, 35\% tabaquistas. No se encontraron diferencias significativas en las características basales de la población (Tabla 1), a excepción de que se encontraron más pacientes con dislipemia en el grupo post-EIHM 
Tabla 4. Objetivos secundarios de evolución intrahospitalaria. FEVI: fracción de eyección del ventrículo izquierdo. ATC: angioplastia transluminal coronaria. DES: stent liberador de drogas. IRA: insuficiencia renal aguda. PCR: paro cardiorrespiratorio. ElHM: estrategias intrahospitalarias de mejora.

\begin{tabular}{lccc} 
& $\begin{array}{c}\text { Pre-EIHM } \\
(\mathrm{n}=63)\end{array}$ & $\begin{array}{c}\text { Post-EIHM } \\
(\mathrm{n}=92)\end{array}$ & $\mathrm{P}$ \\
\hline Killip y Kimball $(\mathrm{n}, \%)$ & $52(82.5 \%)$ & $75(81,5 \%)$ & 0,9 \\
\hline IV & $2(3,1 \%)$ & $4(4,3 \%)$ & \\
\hline FEVI \% (mediana; RIC) & $55(32-77)$ & $50(45-56)$ & 0,014 \\
\hline Revascularización completa $(\mathrm{n}, \%)$ & $17(26,9 \%)$ & $21(22,8 \%)$ & 0,20 \\
\hline ATC con DES (n, \%) & $32(50,7 \%)$ & $83(90,2 \%)$ & $<0.001$ \\
\hline Sangrado TIMI mayor (n, \%) & $4(6,3 \%)$ & $7(7,6 \%)$ & 0,9 \\
\hline Complic. accesos vasculares $(n, \%)$ & $10(15,8 \%)$ & $8(8,6 \%)$ & 0,17 \\
\hline IRA (n, \%) & $2(3,1 \%)$ & $4(4,3 \%)$ & 0,7 \\
\hline Complicaciones mecánicas $(n, \%)$ & $2(3,1 \%)$ & $2(2,1 \%)$ & 0,7 \\
\hline Shock cardiogénico (n, \%) & $6(9,5 \%)$ & $6(6,5 \%)$ & 0,49 \\
\hline PCR & $2(3,1 \%)$ & $8(8,6 \%)$ & 0,16 \\
\hline Muerte (n, \%) & $2(3,1 \%)$ & $2(2,1 \%)$ & 0,7 \\
\hline Arritmias ventriculares $(n, \%)$ & $8(12,6 \%)$ & $11(11,9 \%)$ & 0,8 \\
\hline
\end{tabular}

$(67,4 \%)$ que en el grupo preintervención $(47,6 \%)(p=0,014)$. El 15\% de pacientes presentó antecedentes de cardiopatía isquémica.

No se evidenciaron diferencias en el tratamiento farmacológico instaurado en ambos grupos (Tabla 2). La mayoría de los pacientes fueron tratados con ticagrelor como segundo antiagregante $(75,8 \%)$, mientras que el clopidogrel fue el segundo más elegido (18,8\%). El 93\% de los pacientes recibió tratamiento con aspirina y el 82,5\% con estatinas.

En cuanto al punto final primario, el TPB fue más corto en el grupo en el cual se implementaron las estrategias de mejora intrahospitalaria de forma estadísticamente significativa, con una reducción de la mediana de 13 minutos (55 minutos vs. 42 minutos; $p=0,014$ ). Además, el tiempo ECG-balón ( 85 minutos vs. 70 minutos; $p=0,012$ ) y el tiempo total de isquemia (197 minutos vs. 161,5 minutos; $p=0,02$ ) también se redujeron de forma significativa (Tabla 3).

En cuanto a los objetivos secundarios de evolución intrahospitalaria, no se encontraron diferencias en cuanto a insuficiencia renal aguda, complicaciones mecánicas, arritmias ventriculares o muerte. Hubo mayor tasa de uso de DES en el grupo posimplentación de las ElHM que en el grupo preimplementación (90,2 vs. 50,7\%; $p<0,001)$ (Tabla 4).

El seguimiento se realizó en el 94,19\% de los pacientes, con una mediana de 7 meses (RIC: 1,23-15,7). A los 180 días no hubo diferencias en el punto final combinado de mortalidad, reinfarto y nueva revascularización, ocurriendo en un 27,3\% de pacientes en el grupo pre- y 15,3\% en el grupo post-EIHM (log rank test $\mathrm{p}=0,33$ ). Al evaluar la asociación entre estas medidas y la supervivencia en el seguimiento mediante un modelo de regresión de Cox, se obtuvo un HR de 0,69 (IC95\%: 0,32-1,47; p=0,34) (Figura 1).

\section{DISCUSIÓN}

En este estudio encontramos que la implementación del bypass de la guardia y la preactivación de hemodinamia en pacientes con IAMCEST se asoció a una reducción del tiempo puerta-balón. También se observó una reducción en el tiempo total de isquemia, y una tendencia a la reducción del endpoint secundario combinado de eventos clínicos que no alcanzó significancia estadística.

Según una evaluación realizada por la Organización Mundial de la
Salud y la Organización Panamericana de la Salud', en nuestro país la muerte cardiovascular representa el $29 \%$ de la mortalidad prematura (entre 30 a 69 años) en hombres y el 23\% en mujeres. El 31\% de estas muertes en hombres, y $21 \%$ en mujeres, fueron de causa isquémica. En Argentina, esta entidad tiene una mortalidad intrahospitalaria de 8,8\% en promedio según el mayor y último registro realizado a nivel nacional, el Argen-IAM-ST de 2015, similar a los valores obtenido en el SCAR de 2011, con una mortalidad del 6,2\%, y al registro CONAREC de 2009, con 8,2\%,8. Considerando registros históricos en nuestro país, como el SCAR 2005 en el cual la mortalidad hospitalaria fue de 13,8\%, la mortalidad relacionada al IAMCEST ha disminuido con el desarrollo de técnicas de reperfusión; pero sigue siendo elevada en comparación con registros internacionales (de 4 a $6 \%$ en algunos países europeos ${ }^{9}$ ).

Durante un IAMCEST, hay 3 marcadores principales que definen la magnitud del daño miocárdico: la localización de la lesión (que determinará la cantidad de miocardio en riesgo), el flujo colateral y el tiempo de reperfusión ${ }^{10}$. Otro estudio, ya en el 2004, demostró que por cada 30 minutos de retraso en la angioplastia aumenta 7.5\% la mortalidad ${ }^{11}$, y otro estudio de 30.000 pacientes del 2006 demostró una mortalidad del 3\% con TPB menor a 90 minutos, comparado con 4,2, 5,7 y 7,4 para tiempos de 90-120 minutos, 91-150 minutos y más de 150 minutos, respectivamente ${ }^{12}$.

De todas maneras, el impacto de los retrasos en la reperfusión no es igual para todos los pacientes: un registro de más de 1800 pacientes de Estados Unidos ${ }^{13}$ demostró un aumento progresivo de la mortalidad con el retraso en reperfusión en los pacientes con shock cardiogénico, mientras que en aquellos sin shock la mortalidad se mantuvo sin cambios significativos hasta las 6 horas. En este contexto, las medidas que disminuyan los tiempos de reperfusión tienen potencial de disminuir aún más el tamaño del infarto y los eventos a corto y largo plazo, sobre todo en aquellos pacientes de mayor riesgo.

En cuanto a los tiempos de reperfusión en Argentina, acorde al ya mencionado Argen-IAM-ST 14, la demora entre el inicio del dolor y la consulta médica tuvo una mediana de 170 minutos. La mediana de tiempo puerta-balón fue de 95 minutos, mientras que menos de la mitad de los pacientes tuvieron un tiempo menor a 90 minutos como indican las guías de práctica clínica. El tiempo total de isquemia tuvo una mediana de 300 minutos.

En nuestro país, se encuentra publicada una serie de Mendoza ${ }^{15}$ del año 2016, en el cual se registraron los tiempos de reperfusión de IAMCEST en un centro de dicha provincia, dividiéndolos en 5 etapas: consulta, traslado, guardia, unidad coronaria y hemodinamia. Allí identificaron que el tiempo hasta la consulta representó el 40-50\% de los retrasos, y que el tiempo entre la consulta y la decisión terapéutica representó un $35 \%$ del tiempo si se trataba de un centro periférico derivador y $15 \%$ si fue en el hospital. La mediana de tiempo total de isquemia en dicha serie fue de 303 minutos, y del tiempo puerta-balón de 107 minutos. En el registro CathPCl de Estados Unidos, que abarca más de 1500 hospitales a lo largo de todo el país, con más de 600.000 pacientes incluidos en el 2014, un 93,5\% de los pacientes que consultaron a un centro con capacidad de ATCp tuvieron un tiempo puerta-balón menor a 90 minutos, mientras que en aquellos derivados desde un centro sin hemodinamia solo un 32,3\% alcanzaron este objetivo ${ }^{16}$.

Otro estudio de Estados Unidos demostró que dentro de 28 posibles 


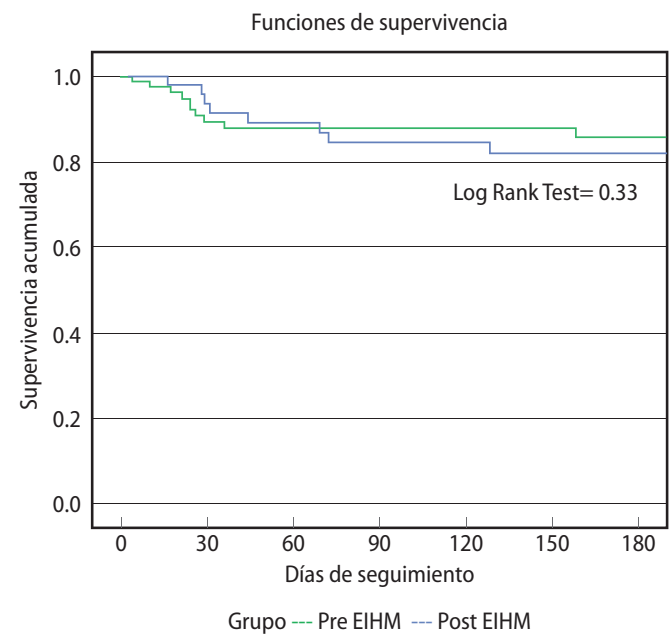

Figura 1. Curvas de supervivencia para punto final combinado de mortalidad, reinfarto y nueva revascularización. ElHM: estrategias intrahospitalarias de mejora.

estrategias para disminuir estos tiempos, 6 se asociaron de forma significativa a una reducción eficaz ${ }^{17}$. Estas fueron: preactivación de hemodinamia por staff de emergencias, preactivación a través de un único llamado a un operador central, preparación de la sala de hemodinamia mientras el paciente se encuentra en camino al centro, llegada de hemodinamistas en menos de 20 minutos tras el llamado, tener un cardiólogo 24 hs en el centro, y el uso de feedback en tiempo real entre la sala de emergencias y la de hemodinamia.

Desde el años 2015 la Argentina está participando de la Iniciativa Stent-Save a Life ${ }^{18}$, programa creado por la Sociedad Europea de Cardiología, el EuroPCR y la Asociación Europea de Intervenciones Cardiovasculares Percutáneas (EAPCI) cuya misión es mejorar el acceso de los pacientes con IAMCEST a un tratamiento de reperfusión de calidad y dentro de los tiempos recomendados por las guías clínicas El ICBA es uno de los 45 centros del país que se encuentran formando parte de su programa de mejora de tiempos puerta-balón.

En cuanto a las limitaciones de nuestro trabajo, al ser un estudio retrospectivo no calculamos la potencia de nuestra muestra para nuestro punto final primario. Los puntos finales secundarios deben además tomarse como exploratorios, dado que no podemos establecer causalidad directa ni contamos con la cantidad de pacientes necesarios para evaluarlo correctamente. También debemos tener en cuenta que existen muchos factores no evaluados que también pueden influir sobre los tiempos puerta-balón y total de isquemia en los años estudiados, como por ejemplo diferentes realidades del país en cuanto a redes de ambulancias. Al estar en un centro monovalente de alta complejidad con hemodinamia las 24 hs, es posible que nuestros tiempos no reflejen la realidad del país. Muy probablemente los retrasos en reperfusión de nuestra institución obedecen principalmente a cuestiones internas, mientras que en otros centros respondan más a las dificultades extrahospitalarias. Para poder implementar estas estrategias a nivel país, es necesario contar con redes de atención, con conexión fluida entre ambulancias, centros con hemodinamia y centros sin hemodinamia y con protocolos estrictos que logren que se pierda el menor tiempo posible, y así poder disminuir los eventos de nuestros pacientes.

\section{CONCLUSIÓN}

En este estudio observacional retrospectivo unicéntrico, la implementación de estrategias intrahospitalarias de mejora, como la preactivación de hemodinamia y el bypass de la guardia, se asociaron de forma significativa con la reducción de los tiempos de reperfusión en el IAMCEST, con una tendencia a la reducción de eventos cardiovasculares mayores.

\section{BIBLIOGRAFÍA}

1. OPS/OMS, Situación de Salud en las Américas: Indicadores Básicos 2013. Washington, D.C. Estados Unidos de América, 2013.

2. Terkensen CJ, Sørensen JT, Maeng M, Jensen LO, Tilsted HH, Trautner S, et al. Sys tem delay and mortality among patients with STEMI treated with primary percutaneous coronary intervention JAMA 2010:304(7):763-771.

3. Guerchicoff A, Brener SJ, Maehara A, Witzenbichler B, Fahy M, Xu K, et al. Impact of Delay to Reperfusion on Reperfusion Success, Infarct Size, and Clinical Outcomes in Patients With ST-Segment Elevation Myocardial Infarction: The INFUSE-AMI Trial (INFUSE-Anterior Myocardial Infarction). J Am Coll Cardiol Int 2014;7(7):733-740.

4. Lambert L, Brown K, Segal E, Brophy J, Rodes-Cabau J, Bogaty P. Association Between Timeliness of Reperfusion Therapy and Clinical Outcomes in ST-Elevation Myocardial Infarction. JAMA 2010;303(21):2148-2155.

5. Thygesen K, Alpert JS, Jaffe AS, Simoons ML, Chaitman BR, White HD, et al. Third Universal Definition of Myocardial Infarction. Eur Heart J 2012; 33(20):2551-2567

6. Ibanez B, James S, Agewall S, Antunes MJ, Bucciarelli-Ducci C, Bueno H, et al. 2017 ESC Guidelines for the management of acute myocardial infarction in patients presenting with ST-segment elevation: The Task Force for the management of acute myocardial infarction in patients presenting with ST-segment elevation of the European Society of Cardiology (ESC). Eur Heart J 2018;39(2):119-177

7. Higa C. Comparación temporal de dos registros multicéntricos argentinos de infarto con elevación del segmento ST: SCAR 2011 - ARGEN IAM ST 2015. Presentation presented at; $2016 ; 42^{\circ}$ Congreso Argentino de Cardiología.

8. Pérez GE, Costabel JP, González N, Zaidel E, Altamirano M, Schiavone M et al. Infarto agudo de miocardio en la República Argentina: Registro CONAREC XVII. Rev Argen Cardiol 2013;81(5):390-399.

9. Kristensen SD, Laut KG, Fajadet J, Kaifoszova Z, Kala P, Di Mario C, et al. Reperfusion therapy for ST elevation acute myocardial infarction 2010/2011: current status in 37 ESC countries. Eur Heart J 2014;35(29):1957-70.

10. Christian TF, Schwartz RS, Gibbons RJ. Determinants of infarct size in reperfusion therapy for acute myocardial infarction. Circulation 1992:86(1):81-90.

11. De Luca G, Suryapranata H, Ottervanger JP, Antman EM. Time delay to treatment and mortality in primary angioplasty for acute myocardial infarction: every min ute of delay counts. Circulation 2004:109(10):1223-1225.

12. McNamara RL, Wang Y, Herrin J, Curtis JP, Bradley EH, Magid DJ, et al. Effect of Door-to-Balloon Time on Mortality in Patients With ST-Segment Elevation Myo cardial Infarction. J Am Coll Cardiol 2006:47(11):2180-6

13. Brodie BR, Stuckey TD, Muncy DB, Hansen CJ, Wall TC, Pulsipher M, et al. Importance of time-to-reperfusion in patients with acute myocardial infarction with and without cardiogenic shock treated with primary percutaneous coronary intervention. Am Heart I 2003:145(4):708-715.

14. Gagliardi JA, Charask A, Perna E, D'imperio H, Bono J, Castillo Costa Y, et al. Encuesta Nacional de infarto agudo de miocardio con elevación del ST en la República Argentina (ARGENT-IAM-ST). Rev Fed Arg Cardiol 2017;46(1):15-21

15. Ortego J, Aveni S, Ripa L, Estrella N, Suriano J, Ferraro M, et al. Análisis de tiempos de intento de reperfusión en pacientes con infarto con elevación de ST. Revista CONAREC. 2016:32(137):274-278.

16. Masoudi FA, Ponirakis A, de Lemos JA, Jollis JG, Kremers M, Messenger JC, et al. Trends in U.S. Cardiovascular Care: 2016 Report From 4 ACC National Cardiovascular Data Registries, J Am Coll Cardiol. 2017:69(11):1427-1450.

17. Bradley EH, Herrin J, Wang Y, Barton BA, Webster TR, Mattera JA, et al. Strategies for Reducing the Door-to-Balloon Time in Acute Myocardial Infarction. N Engl Med 2006:355(22):2308-2320

18. Iniciativa Stent - Save a Life! Argentina. Disponible en http://stentsavealife.com ar. Consultado el 20/11/2018. 

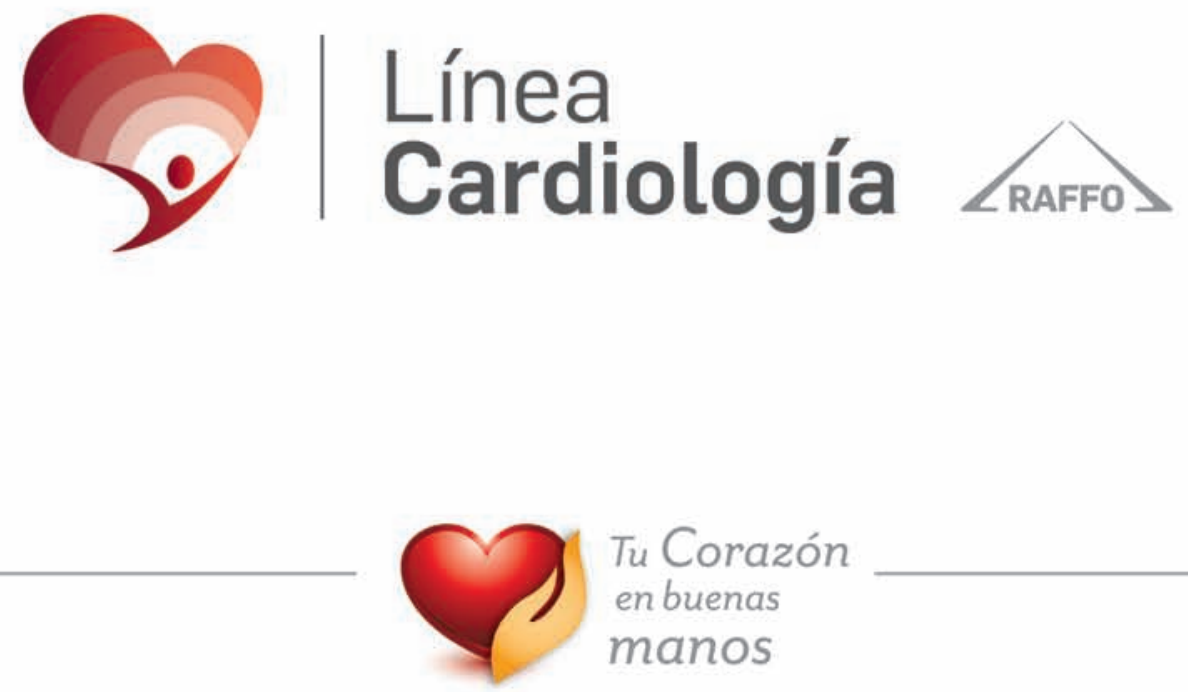

Tu Corazón

en buenas

manos

Nabila

NŌSTER

AMLODIPINA \VALSARTAN

Al Alpertan

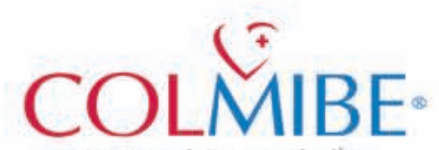

atorvastatina / ezetimibe

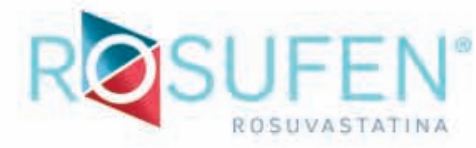

1. LIPIFEN

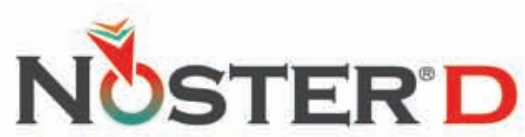

AMLODIPINA / VALSARTAN / HCTZ

Ailpertan:D

Nabratín

(2) ZOLPLLAT 


\title{
TROMBOEMBOLISMO PULMONAR AGUDO EN LA REPÚBLICA ARGENTINA: PROTOCOLO DEL REGISTRO CONAREC XX
}

\author{
ACUTE PULMONARYTHROMBOEMBOLISM IN THE ARGENTINE \\ REPUBLIC: CONAREC XX REGISTRY PROTOCOL
}

\author{
IGNACIO M. CIGALINI', CRISTHIAN E. SCATULARO', JUAN C. JÁUREGUI', MAICO I. BERNAL', JAVIER I. ORTEGO', JUAN M. ABOY', \\ DANIEL CORNEJO', SEBASTIÁN GARCÍA ZAMORA', EZEQUIEL J. ZAIDEL'
}

\begin{abstract}
RESUMEN
Introducción. El tromboembolismo de pulmón agudo (TEP) representa la tercera causa de mortalidad cardiovascular. Sin embargo, existen pocos datos de esta patología en nuestro país.

Objetivo. Describir las características basales, evolución y tratamiento implementado en pacientes internados por TEP agudo en Argentina.

Métodos. Estudio multicéntrico, prospectivo y observacional de pacientes con diagnóstico de TEP agudo internados en centros con residencia de Cardiología desde octubre de 2016 a noviembre de 2017 independientemente si este fuere causa de la internación o surgiera como complicación de hospitalización por otra causa. Se remitirán datos en forma online a través de un sitio seguro con contraseña individual para cada centro participante. Se analizarán variables sociodemográficas, clínicas, estudios complementarios y evolución intrahospitalaria. Se realizará auditoría cruzada al 20\% de los centros.

Conclusiones. El registro CONAREC XX aportará valiosa información para conocer la realidad del TEP agudo en nuestro país.
\end{abstract}

Palabras clave: registros médicos; embolia pulmonar; Argentina.

\section{ABSTRACT}

Introduction: Acute pulmonary thromboembolism (PTE) represents the third cause of cardiovascular mortality. However, there are few data on this pathology in our country.

Objective: To describe the baseline characteristics, evolution and treatment implemented in patients hospitalized for acute PTE in Argentina.

Methods: Multicenter, prospective and observational study of patients diagnosed with acute PTE admitted to centers with cardiology residency from October 2016 to November 2017, regardless of whether this was due to hospitalization or as a complication of hospitalization due to another cause. Data will be sent online through a secure site with an individual password for each participating center. Sociodemographic, clinical variables, complementary studies and in-hospital evolution will be analyzed. Cross audits will be carried out on $20 \%$ of the centers. Conclusions: The CONAREC XX registry will provide valuable information to know the reality of acute PTE in our country.

Keywords: medical records; pulmonary embolism; Argentina.

REVISTA CONAREC 2018;33(146):244-245 | DOI:10.32407/RCON/2018146/0244-0245

\section{INTRODUCCIÓN}

El tromboembolismo de pulmón (TEP) representa la tercera causa de mortalidad cardiovascular', siendo responsable de al menos 100.000 muertes anuales en Estados Unidos ${ }^{2}$ y 300.000 en Europa ${ }^{3}$. Su presentación clínica variable y frecuentemente inespecífica resulta muchas veces un desafío diagnóstico con el consecuente riesgo de infraestimar su real incidencia ${ }^{4}$ e incluso retrasar el inicio de tratamiento específico que resultará en un peor pronóstico. Esta situación determina que se trate de la principal causa de mortalidad intrahospitalaria prevenible 5 .

A pesar de un tratamiento correcto, una significativa proporción de sobrevivientes deberán afrontar consecuencias invalidantes como riesgo aumentado de recurrencia ${ }^{6}$ e hipertensión pulmonar crónica, convirtiendo al TEP en la tercera causa de morbilidad cardiovascular luego de la cardiopatía isquémica y el accidente cerebrovascular? Si bien hay numerosos registros internacionales que han colaborado en conocer factores de riesgo y su historia natura|8-11, existe escasa información en nuestro medio acerca del manejo de esta patología ${ }^{12}$. Al mismo tiempo, a pesar de los avances y el creciente interés en el TEP, continúan existiendo grandes "zonas grises" y controversias especialmen-

\footnotetext{
1. Investigadores registro CONAREC XX
}

$\triangle$ Correspondencia: Dr. Ignacio Manuel Cigalini. Arribeños 3230. 4to piso, departamento 10.C1429BKJ CABA, Rep. Argentina.imcigalini@gmail.com.

Los autores declaran no tener conflictos de intereses.

Recibido: 27/08/2018 | Aceptado: 10/09/2018 te en lo que respecta al tratamiento en su fase aguda, como ser la reperfusión en pacientes con riesgo intermedio-alto o el desarrollo de diferentes métodos de reperfusión endovascular. Asimismo, no contamos con información respecto del impacto generado por la introducción de los anticoagulantes directos orales (ACOD) en la práctica clínica habitual. Estos factores motivaron que desde el Consejo Argentino de Residentes de Cardiología (CONAREC) se inicie un nuevo registro nacional que ayude a comprender cuál es la realidad del TEP en nuestro país en el marco de un programa superador que incluyó actividades de capacitación y difusión para residentes de cardiología y la comunidad en general ${ }^{13}$. El objetivo de este trabajo es describir las características basales, evolución y tratamiento implementado en pacientes internados por TEP agudo en Argentina. Como objetivos secundarios planteamos evaluar terapias de reperfusión de acuerdo a la estratificación de riesgo, identificar predictores de mortalidad intrahospitalaria, validar herramientas clínicas disponibles para estimar riesgo de eventos, evaluar diferencias en la evaluación diagnóstica y terapéutica de acuerdo a servicio responsable y área de internación; y analizar el perfil de pacientes que reciben ACOD en comparación con ensayos clínicos.

\section{MATERIALES Y MÉTODOS}

Estudio observacional, multicéntrico, prospectivo, con seguimiento limitado a la internación, realizado entre octubre de 2016 y noviembre de 2017. Se incluyeron pacientes de 16 años o más, admitidos a unidad coronaria, unidad de terapia intensiva o sala de internación general de centros con residencia de Cardiología afiliados al CONAREC, por episodio de tromboembolis- 
mo pulmonar agudo confirmado independientemente si fuera la causa de internación o surgiera como complicación de la internación por otra causa. Los datos serán recolectados por un médico residente de Cardiología, delegado de cada centro ante CONAREC y subinvestigador del estudio. Se obtendrán a partir de la entrevista personal a cada paciente durante la internación y de datos constatados en la historia clínica. La carga de datos se realizará on-line a través de un electronic case report form (Figura 1) diseñado en la plataforma LimeSurvey ${ }^{\circledR}$ con acceso exclusivo y único para cada centro a partir de una clave individual, con transferencia automática e inmediata a una base central en la plataforma. Para estratificar el riesgo de los pacientes se utilizará la clasificación sugerida por la European Society of Cardiology ${ }^{14}$, el Pulmonary Embolism Severity Score (PESI) $)^{15,16}$ y el PESI simplificado ${ }^{17}$. Asimismo, se evaluarán los puntajes RIETE ${ }^{18}$ y HAS-BLED ${ }^{19}$ para determinar el riesgo de sangrado. El análisis de los puntajes de riesgo se calculará en forma independiente y ciega a partir de las variables que los componen por el investigador principal. Se consideró la clasificación del Bleeding Academic Research Consortium (BARC) ${ }^{20}$ para definir y clasificar los sangrados. El resto de las definiciones se describen en el material suplementario.

Se realizará auditoría cruzada al 20\% de los centros participantes por un comité independiente. Se cotejarán variables aleatorias de la información remitida con la de las historias clínicas de las instituciones.

\section{ANÁLISIS ESTADÍSTICO}

Las variables continuas se expresarán con media y desvío estándar o mediana y rangos intercuartilos, dependiendo de la distribución de las mismas. Las variables categóricas serán expresadas como números y porcentajes. Para las comparaciones entre grupos se utilizará el test de Student o el test de suma de rangos de Wilcoxon, según corresponda.
Las comparaciones entre proporciones se realizará mediante el test de Chi cuadrado o el test exacto de Fisher, dependiendo de la frecuencia de valores esperados. En todos los casos se asumirá un error alfa del 5\% para establecer la significación estadística. Para el análisis estadístico se emplearán los programas estadísticos Epi Info 7.2 y STATA 13.

\section{CONSIDERACIONES ÉTICAS}

Se remitirá protocolo y consentimiento informado al comité de ética de cada centro para su aprobación. Los mismos se encuentran desarrollados en el material suplementario. No se obtendrán datos filiatorios de los pacientes, preservando su identidad.

\section{AUTORÍA}

La publicación de los resultados y posteriores subanálisis se realizarán bajo la autoría del CONAREC y del Comité Organizador del Registro. A los centros participantes se les entregará una certificación institucional como participantes del Registro y a los investigadores y colaboradores de cada centro se les dará una certificación como coautores del Registro CONAREC XX. Para la utilización de los datos del registro global, los investigadores deberán enviar una presentación con los objetivos del trabajo al Comité Organizador del registro de acuerdo se describe en la página oficial (www.conarec.org). El Comité Organizador será el encargado de administrar el acceso a la base de datos generales, para proteger el valor de la información del registro.

\section{CONCLUSIÓN}

El Registro CONAREC XX aportará información valiosa para conocer la realidad de los pacientes con TEP agudo atendidos en centros con residencias de cardiología de nuestro país.

\section{BIBLIOGRAFÍA}

1. Heit J. The epidemiology of venous thromboembolism in the community. Arterioscler Thromb Vasc Biol 2008:28(3):370-372

2. Horlander KT, Mannino DM, Leeper KV. Pulmonary embolism mortality in the United States, 1979-1998: an analysis using multiple-cause mortality data. Arch Intern Med 2003:163(14):1711-7.

3. Arya R. Venous Thromboembolism Prevention. A Patient Safety Priority. London: Department of Health; 2009 .

4. Cohen A, Agnelli G, Anderson F, Arcelus J, Bergavist D, Brecht J, et al. Venous thromboembolism (VTE) in Europe. The number of VTE events and associated morbidity and mortality. Thromb Haemost 2007;98(4):756-64.

5. Galson SK. The Surgeon General's Call to Action to Prevent Deep Vein Thrombosis and Pulmonary Embolism. U.S. Department of Health and Human Services; 2008.

6. Eichinger S, Weltermann A, Minar E, Stain M, Schounauer V, Scheider B, et al. Symptomatic pulmonary embolism and the risk of recurrent venous thromboembolism. Arch Intern Med 2004;1 (164):92-96.

7. Consenso de Enfermedad Tromboembólica. Consenso Argentino SAC. Rev Argent Cardiol 2009:77:411-28

8. Pollack C, Schreiber D, Goldhaber S, Slattery D, Fanikos J, O'Neil B, et al. Clinical characteristics, management, and outcomes of patients diagnosed with acute pulmonary embolism in the emergency department: initial report of EMPEROR (Multicenter Emergency Medicine Pulmonary Embolism in the Real World Registry). J Am Coll Cardiol 2011;57(6):700-706.

9. Laporte S, Mismetti P, De'cousus H, Uresandi F, Otero R, Lobo J, et al. Clinical predictors for fatal pulmonary embolism in 15,520 patients with venous thromboembolism: findings from the Registro Informatizado de la Enfermedad Tromboembólica venosa (RIETE) Registry. Circulation 2008:117(13):1711-1716.

10. Goldhaber S, Visani L, De Rosa M. Acute pulmonary embolism: clinical outcomes in the International Cooperative Pulmonary Embolism Registry (ICOPER). Lancet 1999;117(13):1386-1389.
11. Kasper W, Konstantinides S, Geibel A. Management strategies and determinants of outcome in acute major pulmonary embolism: results of a multicenter registry. J Am Coll Cardiol 1997;30:1165-1171.

12. Castillo Costa Y, Gómez Santa María H, Mauro V, Blanco Cerdeira P, Thierer J, Higa C. Registro Argentino de Enfermedad Tromboembólica Venosa. Rev Argent Cardiol 2013;81:251-257.

13. Scatularo CE. Tratamiento del tromboembolismo pulmonar agudo: controversias actuales. Rev Conarec 2017:(138):30-39.

14. Kostantinides SV, Torbicki A, Agnelli G, Danchin N, Fitzmaurice D, Galie N, et al. 2014 ESC Guidelines on the diagnosis and management of acute pulmonary embolism. Eur Heart J 2014;35(43):3033-3080.

15. Aujesky D, Obrosky D, Ra S, Auble T, Perrier A, Cornuz J, et al. Derivation and validation of a prognostic model for pulmonary embolism. Am J Respir Crit Care Med 2005;172(8):1041-6.

16. Donzé J, Le Gal G, Fine M, Roy P, Sanchez O, Verschuren F et al. Prospective validation of the Pulmonary Embolism Severity Index. A clinical prognostic model for pulmonary embolism. Thromb Haemost 2008;100(5):943-8.

17. Jiménez D, Aujesky D, Moores L, Gómez V, Lobo J, Uresandi F, et al. Simplification of the pulmonary embolism severity index for prognostication in patients with acute symptomatic pulmonary embolism. Arch Intern Med 2010;170(9):1383-9.

18. Ruíz-Giménez N, Suárez C, González R, Nieto J, Todolí J, Samperiz A, et al. Predictive variables for major bleeding events in patients presenting with documented acute venous thromboembolism. Findings from the RIETERegistry. Thromb Haemost 2008;100(1):26-31.

19. Kooiman J, van Hagen N, I lesias del Sol A, Planken E, Lip G, van der Meer F, et al. The HAS-BLED Score Identifies Patients with Acute Venous Thromboembolism at High Risk of Major Bleeding Complications during the First Six Months of Anticoagulant Treatment. PLOS ONE 2015:10(4):E0122520.

20. Mehran R, Rao S, Bhatt D, Gibson C, Caixeta A, Eikelboom J, et al. Standardized Bleeding Definitions for Cardiovascular Clinical Trials. A Consensus Report From the Bleeding Academic Research Consortium. Circulation 2011;123(23):2736-2747. 


\title{
SCORE DE GOLDMAN MODIFICADO PARA LA EVALUACIÓN DE PACIENTES CON DOLOR PRECORDIAL EN LA GUARDIA CENTRAL
}

\author{
GOLDMAN INDEX MODIFIED FOR THE EVALUATION OF PATIENTS WITH \\ PRECORDIAL PAIN IN THE CENTRAL ER
}

\section{CARLA VALLEJOS OLMOS ${ }^{1}$, JUAN P. RICARTE-BRATTI2 ${ }^{2}$ JULIO O. BONO ${ }^{3}$, EDUARDO MOREYRA ${ }^{4}$}

\section{RESUMEN}

Introducción. El score de Goldman modificado ha demostrado efectividad en aquellos pacientes que consultan a la guardia por dolor precordial. A pesar de ello, sigue siendo poco usado como herramienta para el alta precoz en combinación con una sola muestra troponina T ultrasensible (TnT US).

Objetivo. Evaluar la especificidad, sensibilidad, el valor predictivo negativo (VPN) y el valor predictivo positivo (VPP) del score de Goldman modificado y de la TnT US al ingreso. Materiales y métodos. Estudio prospectivo descriptivo de pacientes que acudieron por guardia central con dolor precordial a quienes se les realizó electrocardiograma, dosaje de TnT US y se evaluó el riesgo de enfermedad coronaria a través del puntaje de riesgo de Goldman modificado para establecer un protocolo de alta segura y precoz. Se realizó un seguimiento de los pacientes a los 30 días del alta. Resultados. Se incluyeron 34 pacientes. El score de Goldman modificado fue aplicado a sujetos con dolor precordial con sospecha de síndrome coronario agudo (SCA), con una sensibilidad del 100\%, especificidad del 41\%, VPP $41 \%$ y VPN 100\% para realizar el diagnóstico rápido de exclusión. Para la TnT US hora 0, la sensibilidad y especificidad para SCA fueron del $27 \%$ y del $79 \%$, respectivamente $(p=0,001)$

Conclusión. Este protocolo incorpora un score de evaluación de riesgo estructurado de manera simple con parámetros clínicos y dosaje de troponina T ultrasensible, tiene el potencial de permitir el alta temprana de los pacientes con sospecha de síndrome coronario agudo, y la internación precoz en los que se diagnosticó. Solo en un escaso número de pacientes se debieron realizar otros estudios para aclarar el diagnóstico.

Palabras clave: dolor de pecho; troponina T; síndrome coronario agudo.

\section{ABSTRACT}

Introduction. The modified Goldman Index has shown effectiveness in those patients who consult the ER for precordial pain. Despite this, it is still little used as a tool for early discharge in combination with a single high-sensitivity Troponin T (hs-cTnT) sample.

Objective. To assess the specificity, sensitivity, negative predictive value (NPV) and positive predictive value (PPV) of the modified Goldman Index and hs-cTnT upon admission.

Materials and methods. Descriptive prospective study of patients who attended the ER with precordial pain in whom an electrocardiogram was performed, measurement of hs-cTnT, and the risk of coronary heart disease was assessed through the modified Goldman Index to establish a safe and early discharge protocol. Patients were followed for 30 days after discharge.

Results 34 patients were included. The modified Goldman Index was applied to patients with precordial pain with suspected acute coronary syndrome (ACS) with a sensitivity of $100 \%$, specificity of $41 \%$, PPV $41 \%$ and NPV $100 \%$ to perform the rapid diagnosis of exclusion. For the hs-cTnT hour 0 the sensitivity and specificity for ACS was $27 \%$ and $79 \%$ respectively ( $p=0.001$ )

Conclusion. This protocol incorporates a structured risk assessment score in a simple manner with clinical parameters and hs-cTnT measurement, and it has the potential to allow early discharge of patients with suspected acute coronary syndrome, and early hospitalization in those who were diagnosed. Only in a small number of patients should other studies be carried out to clarify the diagnosis.

Keywords: Chest pain, Troponin T, Acute Coronary Syndrome

REVISTA CONAREC 2018;33(146):246-250 | DOI:10.32407/RCON/2018146/0246-0250

\section{INTRODUCCIÓN}

Los síndromes coronarios agudos (SCA) constituyen la primera causa de muerte en el mundo. La tríada diagnóstica de un SCA se basa en la clínica, el electrocardiograma y el dosaje de marcadores séri-

\footnotetext{
1. Servicio de Cardiología.

2. Cátedra de Farmacología de la Universidad Nacional de Córdoba.

3. Jefe de Unidad Coronaria.

4. Jefe del Servicio de Unidad Cardiovascular.

Sanatorio Allende. Córdoba (Córdoba), Argentina.
}

$\triangle$ Correspondencia:Dra. Carla Vallejos Olmos. Ituzaingó 755, X50001JO Córdoba, provincia de Córdoba, Rep.Argentina.carlavallejos25@gmail.com

Los autores declaran no tener conflictos de intereses.

Recibido: 01/11/2017 | Aceptado: 05/03/2018 $\cos ^{1,2}$. La troponina $(T n)$ es el principal biomarcador de injuria miocárdica en estos pacientes. Sin embargo, la cuantificación de la misma en forma aislada no puede identificar el riesgo de infarto agudo de miocardio (IAM) o SCA que ponen en riesgo la vida del paciente. Un resultado negativo de Tn no excluye un SCA y, por lo tanto, el paciente no puede ser dado de alta en forma segura y temprana teniendo en cuenta el "período ventana", que es el tiempo que demoran las Tn en elevarse en sangre. Se han desarrollado las Tn T e I de alta sensibilidad para la exclusión y confirmación temprana del IAM $\mathrm{IM}^{3}$.

Existen además los sistemas de riesgo $\mathrm{TIMI}^{4}$, Heart $^{5}$, Goldman modificado ${ }^{6}$ que permiten evaluar la probabilidad pretest de un paciente con dolor precordial. Actualmente, estos se utilizan junto con las Tn seriadas como estrategia diagnóstica. Para predecir pronóstico se utilizan los modelos de riesgo GRACE7, TIMI $^{4}$ o el PURSUIT ${ }^{8}$. Sin embargo, aún en pacientes con baja probabilidad, de acuerdo a los scores y con Tn convencionales negativas, 
Tabla 1. Score de Goldman modificado. Bajo riesgo ( $<1)$ y no bajo riesgo $(\geq 1)$.

\begin{tabular}{lc}
\hline Dolor típico en reposo & 1 \\
\hline Dolor similar a IAM previo & 1 \\
\hline El dolor no se alivia con nitritos (15 min) & 1 \\
\hline Dolor $>60$ min & 1 \\
\hline Dolor que ocurre con más frecuencia & 1 \\
\hline TAS $<100 \mathrm{mmHg}$ & 1 \\
\hline Disnea aguda & 1 \\
\hline Dolor post-IAM o posrevascularización & 1 \\
\hline Total: & \\
\hline
\end{tabular}

IAM: infarto agudo de miocardio. TAS: tensión arterial sistólica.

existe un porcentaje que presentan SCA. Por lo tanto, el VPN convencional sigue lejos de ser el ideal, al ser negativo en algunos pacientes y aumentar la morbimortalidad en otros sin diagnóstico. Por el tiempo que demora en positivarse, la Tn aumenta los costos ya que pacientes sin SCA quedan internados para monitoreo y aquellos con SCA necesitan dosaje seriado, retrasando así su diagnóstico.

Por lo mencionado, la cuantificación de Tn convencionales tiene baja sensibilidad en el momento de la presentación del paciente en la consulta, requiriendo muestras seriadas hasta de 9 horas de iniciados los síntomas ${ }^{9}$. Estudios recientes encontraron mayor sensibilidad con Tn ultrasensible (Tn US), la cual podría mejorar la precisión para descartar o confirmar el diagnóstico de SCA en el momento de la consulta ${ }^{10,11}$

Existe elevación de las Tn US de causa no cardíaca. Un 2\% de la población general y entre un 10 al 20\% de los pacientes que consultan en el departamento de emergencias tienen Tn Us elevada ${ }^{12}$. De estos, en solo la mitad se ha constatado alguna alteración cardíaca, habitualmente con métodos de imagen.

A pesar de haber demostrado su efectividad en pacientes con dolor de pecho en la sala de emergencia, el score de Goldman modificado sigue siendo poco utilizado como una herramienta para el alta precoz en combinación con una sola muestra Tn US ${ }^{13}$.

\section{OBJETIVOS}

\section{OBJETIVO PRIMARIO}

Evaluar la especificidad, la sensibilidad, el valor predictivo negativo y el valor predictivo positivo del score de Goldman modificado y de la troponina T ultrasensible (TnT US) para la exclusión rápida de SCA en pacientes de bajo riesgo al ingreso.

\section{OBJETIVOS SECUNDARIOS}

Evaluar la sensibilidad y la especificidad de la TnT US según los pacientes consulten en forma precoz (dentro de las primeras 2 horas) o tardíamente (posterior a 3 horas).

Evaluar la especificidad y la sensibilidad de la TnT US en el grupo de pacientes con valores menores de 14 ng/dl (valor blanco mínimo).

Evaluar la sensibilidad y la especificidad del delta de las TnT US a la hora.

\section{MATERIALES Y MÉTODOS}

Estudio prospectivo descriptivo que incluyó pacientes de ambos sexos, mayores de 18 años, que consultaron en la Guardia Central del Sanatorio Allende (Nueva Córdoba, Córdoba) por dolor precordial con sospecha de SCA dentro de las primeras 24 horas del inicio del dolor, entre los meses de mayo a octubre del año 2016.

Se excluyeron pacientes con electrocardiograma (ECG) diagnóstico de isquemia aguda de miocardio (depresión del segmento ST > 1 mm o inversión de la onda T o supradesnivel del ST), arritmias (fibrilación auricular, flutter auricular, taquicardia supraventricular, bloqueo completo o de segundo grado, arritmia ventricular recurrente), edad mayor a 80 años, otra causa de dolor precordial diagnosticada al ingreso (embolismo pulmonar, neumonía, disección de aorta), embarazo, insuficiencia renal crónica o con necesidad de hemodiálisis, cualquier condición que eleve las troponinas (exceptuando los SCA), incapacidad para entender el idioma o firmar el consentimiento informado. En caso de hemólisis de la muestra se realizó una nueva extracción.

El estudio se llevó a cabo de acuerdo a los principios de la Declaración de Helsinki y fue aprobado por el Comité de Ética del sanatorio. Todos los pacientes firmaron un consentimiento informado.

Los pacientes ingresados a Unidad Coronaria recibieron tratamiento completo para SCA, se realizó una historia clínica completa, ECG de 12 derivaciones, exámenes de laboratorio de rutina, radiografía de tórax y monitoreo electrocardiográfico continuo con saturación de oxígeno permanente. Cualquier otro estudio complementario o tratamiento se realizó de acuerdo a la evolución clínica del paciente.

\section{PROBABILIDAD Y ESTRATIFICACIÓN DE RIESGO}

Se aplicó en cada paciente el puntaje de riesgo de Goldman modificado (Tabla 1) en el momento de la consulta.

\section{CUANTIFICACIÓN DE LA TNT US}

Se realizó en el laboratorio central del Sanatorio Allende (Nueva Córdoba, Córdoba), mediante la prueba Elecsys de Roche.

Se obtuvo una muestra de sangre en el momento de la consulta por guardia central para la realización de TnT US, una segunda muestra a la hora y una tercera muestra las dos horas de su ingreso (T0, T1 y T2 respectivamente). Además, se solicitó citología y química a criterio del médico que recibió al paciente.

Los pacientes de riesgo bajo e intermedio, fueron evaluados de manera individualizada por el médico a cargo, el cual decidió la necesidad de internación. Mientras que aquellos con riesgo alto de enfermedad coronaria, fueron ingresados a unidad coronaria y el equipo cardiológico decidió según las guías internacionales y protocolos internos el modo de llegar al diagnóstico definitivo.

\section{CINECORONARIOGRAFÍA}

Se realizó solo a los pacientes que ingresaban con diagnóstico de SCA y/o según el criterio del médico tratante, a aquellos con dolor precordial de alto riesgo de enfermedad coronaria. Fueron realizadas por el servicio de Hemodinamia e Intervenciones por cateterismo. 


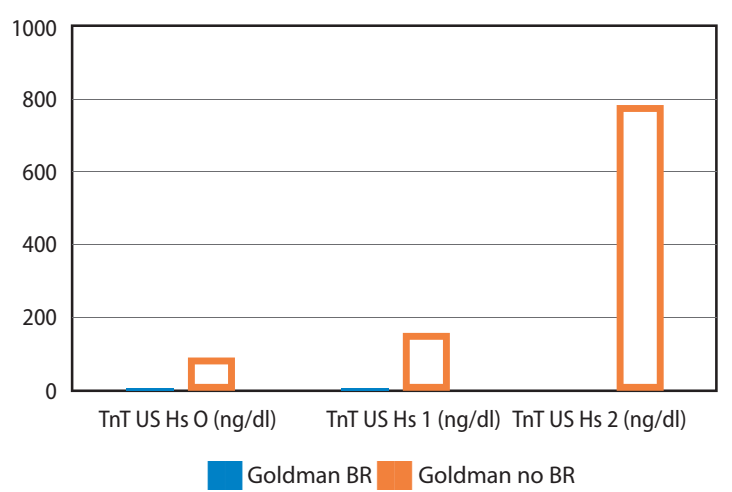

Figura 1. Score de Goldman modificado y el valor de troponina T ultrasensible. Eje de coordenadas x: Valor de TnT US al ingreso (TnT US Hs 0), Valor de Tnt US a la hora del ingreso (Tn US Hs 1), Valor de TnT US a las 2 horas del ingreso (TnT US Hs 2). Eje de coordenadas y: valo entero de TnT US en ng/dl. Goldman BR: score de Goldman modificado de bajo riesgo. Goldman No BR: score de Goldman modificado de no bajo riesgo. TnT US: troponina Tultrasensible.

\section{SEGUIMIENTO}

Los pacientes que fueron dados de alta desde la guardia, ya sea por dosaje de TnT US < 14 ng/dl y/o delta de TnT US de la 1ra hora y de la 2 da hora $<20 \%$, se les realizó seguimiento telefónico a los 30 días para indagar sobre la nueva aparición de angor, progresión del mismo, eventual internación por SCA o revascularización.

\section{ANÁLISIS ESTADÍSTICO}

Las variables categóricas se relacionarán con el método de Fisher o chi cuadrado según corresponda. Las variables continuas con el Test t de Student. Se utilizó el programa estadístico SPSS versión 15.0. Los datos se representarán como media \pm desvío estándar. Se consideraron significativos aquellos valores con $p<0,05$

\section{RESULTADOS}

El total de pacientes incluidos en este estudio fue 34 , siendo el promedio de edad de $56,2 \pm 14,2$ años. El $35 \%(n=12)$ fueron mujeres y el $65 \%(n=22)$ hombres.

De los factores de riesgo para enfermedad coronaria, la hipertensión arterial se presentó en el 47\% ( $n=16)$, la diabetes en el 17,6\% ( $n=6)$, el tabaquismo en el $23,5 \%(n=8)$, la enfermedad carotídea conocida en el $2,9 \%(n=1)$, la dislipidemia en el $41,2 \%(n=14)$ y el antecedente de internación previa por insuficiencia cardíaca en el 2,9\% ( $n=1)$. El $23,5 \%$ ( $n=8)$ de los pacientes presentaba enfermedad coronaria conocida. La fracción de eyección del ventrículo izquierdo mostró una media de $61 \pm 9,8 \%$.

El $29,4 \%(n=10)$ de los pacientes consultaron en las primeras de 3 horas de iniciado el angor. De las características del dolor el $41,1 \%(n=14)$ fue dolor típico, el 32,3\% ( $n=11)$ presentó disnea como equivalente, el $85,3 \%(n=29)$ fue en reposo y el $29,4 \%$ $(n=10)$ tuvo dolor progresivo. Nueve pacientes tomaban ácido acetilsalicílico.

En el ECG, 4 pacientes tenían alteraciones inespecíficas del segmento $S T, 2$ con infradesnivel del segmento $S T<1 \mathrm{~mm}$ y el resto con ECG no diagnóstico.

El $64,7 \%(n=22)$ de los pacientes fueron internados en la Unidad coronaria. De los pacientes sometidos a CCG $(52,9 \%, n=18)$, el $26,5 \%$

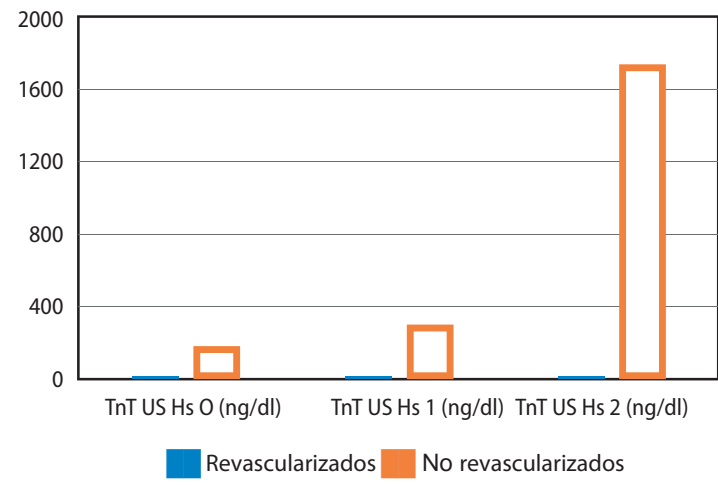

Figura 2. Pacientes con score de Goldman modificado de No Bajo riesgo que fueron revascularizados y no, con los valores de TnT US al ingreso (TnT US hora 0), a la hora (TnT US hora 1) y a las 2 horas (TnT US hora 2). Eje de coordenadas X: Valor de TnT US al ingreso (TnT US Hs 0), Valor de TnT US a la hora del ingreso (TnT US Hs 1), Valor de TnT US a las 2 horas del ingreso (TnT US Hs 2). Eje de coordenadas Y: valor entero de TnT US en ng/dI. TnT US: troponina T ultrasensible.

$(n=9)$ recibió angioplastia coronaria primaria y uno cirugía de revascularización miocárdica. Fueron dados de alta desde la guardia y seguidos telefónicamente a los 30 días (35,3\%, n=12).

El 29,4\% ( $n=10$ ) de los pacientes tenía riesgo bajo según el score de Goldman modificado. De ese total, el dosaje de TnT US al ingreso (TO) tuvo una media de 5,27 $\pm 4,6 \mathrm{ng} / \mathrm{dl}(\mathrm{p}<0,001)$, el dosaje a la 1ra hora (T1) de 5,3 $\pm 4,2 \mathrm{ng} /$ $\mathrm{dl}(\mathrm{p}=0,01)$ y el dosaje a la $2 \mathrm{da}$ hora (T2) de 7,33 $\pm 7,7 \mathrm{ng} / \mathrm{dl}(\mathrm{p}=0,049)$. En este grupo de pacientes (bajo riesgo de score de Goldman modificado) no hubo evidencia de enfermedad coronaria y sólo uno tuvo TnT US positiva. Ninguno requirió revascularización

El 70,6\% (24) de los pacientes presentó un score de Goldman modi-

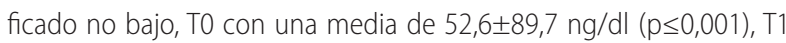
media de $114,9 \pm 223 \mathrm{ng} / \mathrm{dl}(\mathrm{p}=0,01)$ y T2 media de $421,9 \pm 1400 \mathrm{ng} /$ $\mathrm{dl}(\mathrm{p}=0,049)$ (Figura 1). Del total, 10 pacientes $(41,7 \%)$ requirieron revascularización miocárdica (1 cirugía de revascularización miocárdica y 9 angioplastia coronaria primaria). Para este subgrupo de pacientes, los valores de TnT US (T0, T1 y T2) fue: 106 $\pm 120 \mathrm{ng} / \mathrm{dl}(\mathrm{p}=0,039)$, $256 \pm 297 \mathrm{ng} / \mathrm{dl}(\mathrm{p}=0,030)$ y $1063 \pm 2157 \mathrm{ng} / \mathrm{dl}(\mathrm{p}=0,18)$, respectivamente. Los otros 14 tuvieron T0, T1 y T2 de 14,1 $\pm 18,6$ ng/dl, 13,8 $\pm 14,3$ $\mathrm{ng} / \mathrm{dl}$ y $9,8 \pm 9,3 \mathrm{ng} / \mathrm{dl}$ respectivamente todos con $\mathrm{p} \geq 0,05$. El delta de TnT US en este grupo fue del $2 \%$ a la primera hora y del $43 \%$ a la segunda hora, sin eventos en el seguimiento (Figura 2)

De los pacientes con score de Goldman modificado de no bajo riesgo con TnT US negativa ( $n=13)$, el 23\% ( $n=3$ ) requirió revascularización. Mientras que en este mismo grupo, los que tuvieron TnT US positiva al ingreso $(n=11)$, el $63 \%(n=7)$ requirió revascularización.

El score de Goldman modificado fue aplicado a pacientes con dolor precordial con sospecha de SCA, presentando una sensibilidad de 100\%, una especificidad del 41,6\%, un VPP del $41,6 \%$ y un VPN de $100 \%$. Para la T0, la sensibilidad y especificidad ante un SCA fue del $26,9 \%$ y del $79,1 \%$ respectivamente.

El 29,4\% ( $n=10)$ de los pacientes consultaron de manera precoz (antes de las 3 horas de iniciados los síntomas). De ese total, 7 fueron de bajo riesgo y 3 de alto riesgo según el score de Goldman modificado. Ninguno presentó evidencia de enfermedad coronaria. Se evaluó el delta de TnT US en este grupo de pacientes, para el grupo de bajo riesgo a la 1ra hora la media fue de $15 \pm 13 \%$ mientras que a la 2 da hora no hubo variación. 


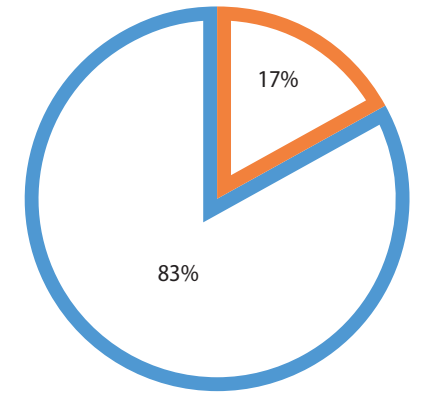

TnT US positiva

TnT US Negativa

Figura 3. Porcentaje de pacientes que fueron revascularizados según el valor cualitativo de la troponina T ultrasensible al ingreso (valor de corte de la TnT US 14 ng/dl). TnT US: troponina Tultrasensible.

El 69,6\% de los pacientes consultó luego de las 3 horas del inicio de los síntomas. De ese total, 4 fueron de bajo riesgo y 20 de no bajo riesgo. Del grupo de no bajo riesgo, los que tuvieron diagnóstico de

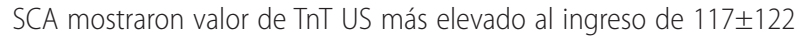
$\mathrm{ng} / \mathrm{dl}(\mathrm{p}=0,028)$, a la hora de $284 \pm 301 \mathrm{ng} / \mathrm{dl}(\mathrm{p}=0,026)$, con delta en ese lapso de $167 \pm 179 \%(p=0,026)$. A las dos horas el valor de TnT US ni el delta fueron significativos (Figura 3).

Del grupo de pacientes que presentó un valor de TnT US $<14$ ng/dl $(n=22)$, todos con score de Goldman modificado de no bajo riesgo, 3 requirieron revascularización ( $p=N S$ ). Mientras que aquellos con TnT US > 14 ng/dl ( $n=12)$ y score de Goldman modificado de no bajo riesgo $(n=11), 6$ recibieron angioplastia coronaria y 1 cirugía de revascularización miocárdica (Figuras 4 y 5).

En solo en un paciente con score de Goldman modificado de bajo riesgo y TnT US >14 ng/dl no se evidenció enfermedad coronaria, presentando un delta de TnT US $<20 \%$ y ningún evento en el seguimiento.

De los pacientes con diabetes ( $n=6), 4(66 \%)$ mostraron enfermedad coronaria y recibieron angioplastia coronaria, formando parte del grupo de no bajo riesgo. Este porcentaje fue significativamente más alto que en pacientes no diabéticos $(p=0,035)$.

Hubo 8 pacientes con enfermedad coronaria previa como antecedente, $3(37,5 \%)$ de los cuales requirieron revascularización, sin existir diferencias con respecto a pacientes sin antecedentes de coronariopatía $(p=0,572)$. Estos pacientes pertenecían al grupo score de Goldman modificado de no bajo riesgo con valor de TnT US bajo con delta $>20 \%$.

\section{DISCUSIÓN}

En nuestro estudio encontramos que el score de Goldman modificado predice de manera exitosa la necesidad de no revascularización en todos los pacientes con bajo riesgo, independientemente de la TnT US, con una sensibilidad del 100\%, una especificidad del $41 \%$, un VPP $41 \%$ y un VPN 100\%. Estos resultados no muestran diferencia en cuanto a la llegada precoz o tardía del paciente a la guardia central. Es para resaltar la variación de los valores de TnT US entre la primera y la segunda muestra, con lo que se podría inferir que sería seguro tomar sólo dos extracciones con diferencia de 1 hora en pacientes de bajo riesgo.

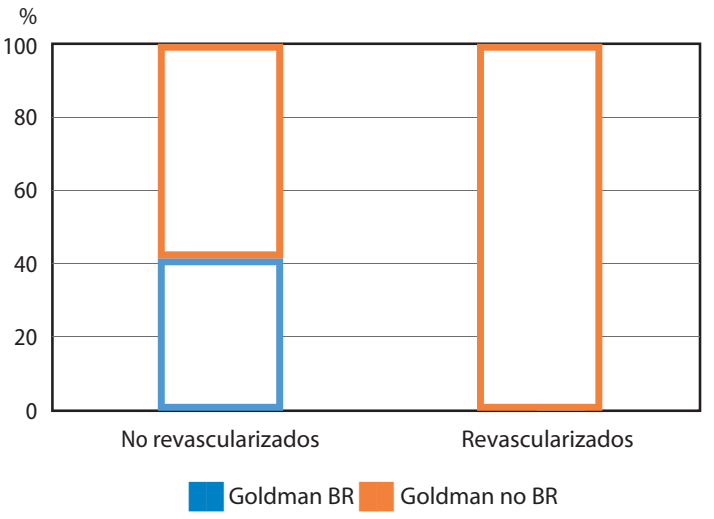

Figura 4. Estratificación de pacientes según el score de Goldman modificado y el porcentaje de revascularizados y no revascularizados. BR: bajo riesgo.

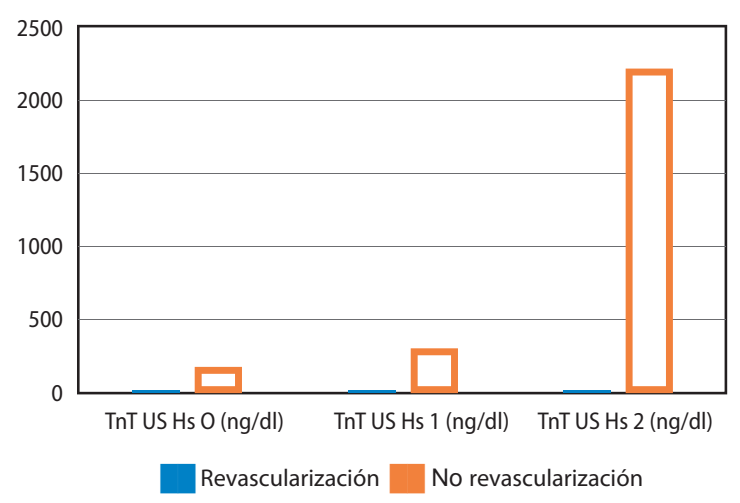

Figura 5. Pacientes del grupo de revascularizados/no revascularizados y los valores de TnT ultrasensible al ingreso (TnT US Hs 0), a la hora del ingreso (TnT US Hs 1) y a las 2 horas del ingreso (TnT US Hs 2). Eje de coordenadas $x$ : valor de TnT US al ingreso (TnT US Hs 0), valor de TnT US a la hora del ingreso (TnT US HS 1), valor de TnT US a las 2 horas del ingreso (TnT US Hs 2). Eje de coordenadas Y: valor entero de TnT US en ng/dl. TnT US: troponina T ultrasensible. a la hora del ingreso (TnT US Hs 1), valor de TnT US a las 2 horas del ingreso (TnT US Hs 2). Eje de coordenadas Y: valor entero de TnT US en ng/dl. TnT US: troponina Tultrasensible.

Un estudio observacional prospectivo evaluó la capacidad de la triage para el alta usando Tn US (TRUST) $)^{15}$ y un protocolo diagnóstico acelerado (ADP) que incorporaba además un ECG sin isquemia y el score de Goldman para identificar a los pacientes de bajo riesgo con sospecha de SCA. EI TRUST ADP identificó pacientes para el alta temprana, el 39,8\% frente a $29,3 \%$ (Tn US <5 ng/l) y 7,9\% (<3 ng/l) $(p<0,001)$, especificidad 43,3\% (IC95\%: 42,7\%-43,4\%) vs 32,0\% (IC95\%: 31,5\%$32,0 \%$ ) y $8,6 \%$ (IC95\%: 8,1\%-8,6\%), respectivamente.

Los resultados de este trabajo sugieren que la introducción de este protocolo tiene el potencial de reducir la duración de la estancia para los pacientes de bajo riesgo (en la actualidad 5 horas, en nuestra institución) después de 2 muestras de TnT US basada en el score de Goldman modificado y el ECG. Además, puede tener beneficios significativos para los servicios de salud mediante la reducción de las tasas de ingresos hospitalarios, el hacinamiento en la sala de guardia, el tiempo del personal y el uso de recursos. En un descriptivo realizado en Reino Unido sobre pacientes con dolor precordial que se presentaron en el departamento de emergencias puso en evidencia el elevado número de ingresos hospitalarios por este motivo de consulta, de los cuales un tercio de la asistencia y la mitad de las admisiones tuvieron un diagnós- 
tico clínico de SCA sin cambios en el ECG, por lo que nos hace pensar que nuestro protocolo mejoraría sustancialmente la atención ${ }^{15}$.

Si tomamos el combinado TnT US más score de Goldman modificado y revascularización, encontramos en nuestra muestra que el 33,3 $\%$ de los pacientes con score alto y TnT US negativas podría tener un evento. Si bien la muestra es pequeña, el adicionarle el mencionado score a la conocida sensibilidad y especificidad de la TnT US en paciente de alto riesgo cardiovascular ${ }^{16}$ suma un gran aporte tanto para el ingreso temprano como para el alta rápida.

A la inversa del escenario clínico anterior, en el caso de un score de Goldman modificado de bajo riesgo con TnT US > 14 ng/dl, lo cual se evidenció en un paciente que no presentó enfermedad coronaria y tuvo un delta de TnT US en la $1^{\circ}$ y $2^{\circ}$ hora negativo. Este punto es comparable con el estudio donde se buscó validar a la troponina I a través de una estrategia diagnóstica de dos horas en pacientes de bajo riesgo según el score TIMI 1,17 . Eventualmente, sin el score este tipo de pacientes son habitualmente internados o por lo menos observados en la sala de emergencias para el dosaje seriado de TnT US, con el aumento de los gastos y eventualmente el sometimiento a estudios invasivos, muchas veces innecesarios.

Aunque en nuestro centro no está validada la estrategia en el cual se cuantifica la TnT US en niveles indetectables ( $5 \mathrm{ng} / \mathrm{l}$ ) en quienes tienen baja probabilidad clínica y ausencia de signos de isquemia en el
ECG, demostró en el seguimiento a 30 días, ser un protocolo seguro para descartar infarto de miocardio o muerte en pacientes dados de alta directamente desde el departamento de emergencias ${ }^{18}$.

La principal limitación de nuestro estudio es el escaso número de pacientes y la ausencia de eventos cardiovasculares en el seguimiento a los 30 días.

Los SCA, como se mencionó anteriormente, son la primera causa de muerte de todo el mundo. Por lo tanto, tener la certeza de su diagnóstico o de su exclusión es importante no sólo para disminuir la morbimortalidad a corto y largo plazo, sino también para disminuir los costos de internaciones y estudios a aquellos pacientes que no lo necesitan, incluyendo el cateterismo coronario, con las complicaciones que los procedimientos invasivos presentan.

\section{CONCLUSIÓN}

Este protocolo, que incorpora un score de evaluación de riesgo estructurado y simple asociado a parámetros clínicos y al dosaje de troponina T ultrasensible, tiene el potencial de permitir el alta temprana en casi todos los pacientes que ingresan con sospecha de síndrome coronario agudo con score de Goldman modificado bajo, la internación precoz de aquellos con diagnóstico de síndrome coronario agudo y, solo en un escaso número de sujetos, realizar otros estudios para aclarar el diagnóstico.

\section{BIBLIOGRAFÍA}

1. Thygesen K Alpert IS, White HD. Universal definition of myocardial infarction. Joint ESC/ACCF/AHAMHF/ Task Force for the Redefinition of Myocardial Infarction. J Am Coll Cardiol 2007;50(22):2173-95.

2. Thygesen K, Mair J, Katus H, Plebani M, Venge P, Collinson P et al. Recommenda tions for the use of cardiac troponin measurement in acute cardiac care. Study Group on Biomarkers in Cardiology of the ESC Working Group on Acute Cardiac Care. Eur Heart J 2010;31(18):2197-204.

3. Body R, Carley S, McDowell G, Jaffe AS, France M, Cruickshank K et al. Rapid exclusion of acute myocardial infarction in patients with undetectable troponin using a high-sensitivity assay. J Am Coll Cardiol 2011:58(13):1332-9.

4. Antman EM, Cohen M, Bernink PJ, McCabe CH, Horacek T, Papuchis G et al. The TIMI risk score for unstable angina/non-ST elevation MI: A method for prognostication and therapeutic decision making. JAMA 2000;284(7):835-42.

5. Backus BE, Six AJ, Kelder JC, Bosschaert MA, Mast EG, Mosterd A et al. A prospective validation of the HEART score for chest pain patients at the emergency department. Int J Cardiol 2013;168(3):2153-8.

6. Carlton EW, Cullen L, Than M, Greaves K. A modified goldman risk score in combination with high-sensitivity troponin proves superior to TIMI in the evaluation of suspected acute cardiac chest pain. J Am Coll Cardiol 2014;63(12):A80.

7. Elbarouni B, Goodman SG, Yan RT, Welsh RC, Kornder JM, Deyoung JP et al. Validation of the Global Registry of Acute Coronary Event (GRACE) risk score for in-hospital mortality in patients with acute coronary syndrome in Canada. Am Heart J 2009;158(3):392-9.

8. Boersma E, Pieper KS, Steyerberg EW, Wilcox RG, Chang WC, Lee KL et al. Predictors of outcome in patients with acute coronary syndromes without persistent ST-segment elevation. Results from an international trial of 9461 patients. The PURSUIT Investigators. Circulation 2000;101(22):2557-67.

9. Keller T, Zeller T, Ojeda F, Tzikas S, Lollpopp L, Sinning C et al. Serial changes in highly sensitive troponin I assay and early diagnosis of myocardial infarction. JAMA 2011:306(24):2684-93.
10. Reichlin T, Hochholzer W, Bassetti S, Steuer S, Stelzig C, Hartwiger S et al. Early diagnosis of myocardial infarction with sensitive cardiac troponin assays. N Engl J Med 2009;361(9):858-67.

11. Hamm CW Goldmann BU, Heeschen C Kreymann G, Berger I Meinertz T. Emergency room triage of patients with acute chest pain by means of rapid testing for cardiac troponin T or troponin I. N Engl J Med 1997:337(23):1648-53

12. Reichlin T, Hochholzer W, Bassetti S, Steuer S, Stelzig C, Hartwiger S et al. Early diagnosis of myocardial infarction with sensitive cardiac troponin assays. N Engl J Med 2009;361(9):858-67.

13. Keller T, Zeller T, Peetz D, Tzikas S, Roth A, Czyz E et al. Sensitive troponin I assay in early diagnosis of acute myocardial infarction. N Engl J Med 2009;361(9):868877.

14. de Lemos JA, Drazner MH, Omland T, Ayers CR, Khera A, Rohatgi A et al. Association of troponin $T$ detected with a highly sensitive assay and cardiac structure and mortality risk in the general population. JAMA 2010;304(22):2503-12. Carlton EW, Cullen L, Than M, Gamble J, Khattab A, Greaves K. A novel diagnostic protocol to identify patients suitable for discharge after a single high-sensitivity troponin. Heart 2015:101(13):1041-6.

15. Chase M, Robey JL, Zogby KE, Sease KL, Shofer FS, Hollander JE. Prospective validation of the Thrombolysis in Myocardial Infarction risk score in the emergency department chest pain population. Ann Emerg Med 2006;48(3):252-9.

16. Goodacre S, Cross E, Arnold J, Angelini K, Capewell S, Nicholl J. The health care burden of acute chest pain. Heart 2005:91(2):229-30.

17. Cullen L, Mueller C, Parsonage WA, Wildi K, Greenslade JH, Twerenbold R. Validation of high-sensitivity troponin I in a 2-hour diagnostic strategy to assess 30-day outcomes in emergency department patients with possible acute coronary syndrome. J Am Coll Cardiol 2013;62(14):1242-1249. 


\title{
TROMBOEMBOLISMO PULMONAR RECURRENTE EN UN PACIENTE CON SÍNDROME ANTIFOSFOLIPÍDICO Y TROMBOCITOPENIA INDUCIDA POR HEPARINA
}

\author{
RECURRENT PULMONARY THROMBOEMBOLISM IN A PATIENT \\ WITH ANTIPHOSPHOLIPID SYNDROME AND THROMBOCYTOPENIA \\ INDUCED BY HEPARIN
}

\section{NAHUEL LITWAK' ${ }^{1}$ MILAGROS SEIJO²}

\begin{abstract}
RESUMEN
El tromboembolismo pulmonar es una urgencia cardiovascular de pronóstico variable y en su estudio etiológico debemos buscar los factores que puedan influir en su génesis y precipitar resultados adversos. La trombocitopenia inducida por heparina y el síndrome antifosfolipídico son enfermedades hematológicas que pueden participar en la fisiopatogenia de tromboembolismo pulmonar y complicar su evolución. A continuación presentamos un caso caso demuestra la ocurrencia de ambas entidades en un paciente.
\end{abstract}

Palabras clave: embolia pulmonar; trombocitopenia; síndrome antifosfolipídico; anticoagulantes.

\section{ABSTRACT}

Pulmonary thromboembolism is a cardiovascular emergency with variable prognosis and in its etiological study we must look for the factors that may influence its genesis and precipitate adverse results.

Heparin-induced thrombocytopenia and the antiphospholipid syndrome are hematological diseases that can participate in the pathophysiology of pulmonary thromboembolism and complicate its evolution. In both cases, they are immune-mediated phenomena that generate a systemic prothrombotic state and require an accurate therapeutic approach.

Keywords: pulmonary embolism; thrombocytopenia; antiphospholipid syndrome; anticoagulants.

REVISTA CONAREC 2018;33(146):251-254 | DOI:10.32407/RCON/2018146/0251-0254

\section{INTRODUCCIÓN}

La trombocitopenia inducida por heparina (HIT) y el síndrome antifosfolipídico (SAF) son enfermedades hematológicas que pueden participar en la fisiopatogenia del tromboembolismo pulmonar (TEP) y agravar su evolución. La coexistencia de estas dos entidades es infrecuente. Se trata en ambos casos de fenómenos inmunomediados que generan un estado sistémico protrombótico. La evidencia relacionada con el abordaje y tratamiento de dichos pacientes es escasa y basada principalmente en reportes de casos y trabajos con series pequeñas de pacientes.

\section{DESARROLLO}

Se presenta el caso de un paciente masculino de 39 años, extabaquista y con antecedente de trombosis venosa profunda (TVP) en el año

\footnotetext{
1 Residente de Cardiología

2 Jefe de Residentes. Hospital Universitario Fundación Favaloro. CABA, Rep. Argentina.
}

$\triangle$ Correspondencia: Dr. Nahuel Litwak. Av. Santa Fe 2534. C1425BGN CABA, Rep. Argentina.nahuel.litwak@gmail.com.

Los autores declaran no tener conflictos de intereses.

Recibido: 10/11/2017| Aceptado: 02/02/2018
2002 por la cual recibió tratamiento anticoagulante con antagonistas de la vitamina K (AVK). Se internó inicialmente en otro centro por encontrarse sintomático para disnea en clase funcional (CF) III asociado a 2 episodios autolimitados de hemoptisis. En dicho centro se realizó diagnóstico de TVP y TEP agudo por lo que recibió tratamiento con heparina sódica. Presentó en el curso de su internación plaquetopenia que alcanzó valores de hasta $37 \mathrm{mil} / \mathrm{mm}^{3}$, interpretándose cuadro como probable HIT, sin disponer de pruebas confirmatorias. Se completó su estudio mediante centellograma ventilación/perfusión que informó ausencia de perfusión pulmonar izquierda y defectos segmentarios de la perfusión pulmonar derecha, con alta probabilidad de TEP. Se realizó cateterismo pulmonar con presión arterial pulmonar media de $47 \mathrm{mmHg}$ y resistencias pulmonares elevadas de 29 unidades Wood. Los análisis de laboratorio con factor antinuclear y serologías virales fueron negativas. Luego de 33 días de internación, se otorgó su egreso hospitalario bajo anticoagulación con acenocumarol y tratamiento de la hipertensión pulmonar con sildenafil y bosentán.

Posterior a su externación, fue evaluado en consultorios externos por persistir con disnea CF III, para descartar hipertensión pulmonar (HTP) tromboembólica crónica. Previo a la realización de los exámenes complementarios solicitados, presentó nuevamente 2 episodios de hemoptisis autolimitados asociados a progresión de su disnea hasta CF IV estando bajo tratamiento anticoagulante. Se realizó angiotomografía computarizada de tórax (Figuras 1 y 2) que informó la presencia de defectos de relleno endovascular en rama segmentaria del ló- 


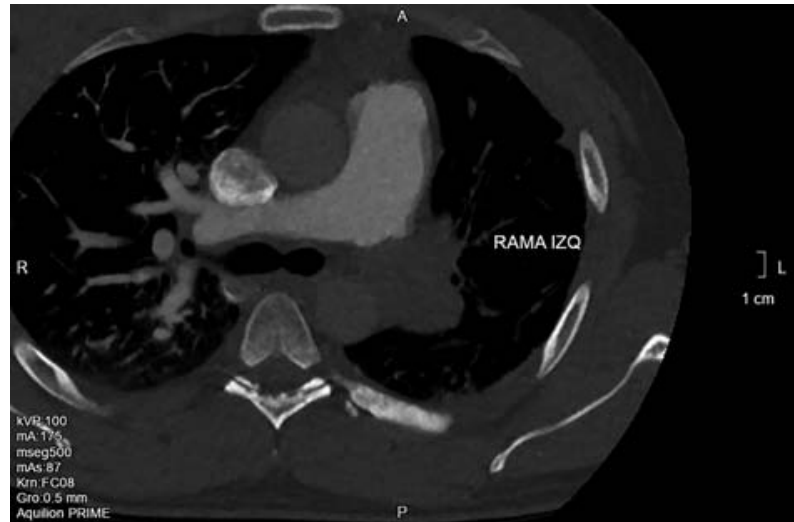

Figura 1. Tomografía computarizada con contraste endovenoso. Se evidencia dilatación del tronco de la arteria pulmonar asociado a defecto de relleno de contraste en la rama izquierda de la misma. Izq: izquierda.

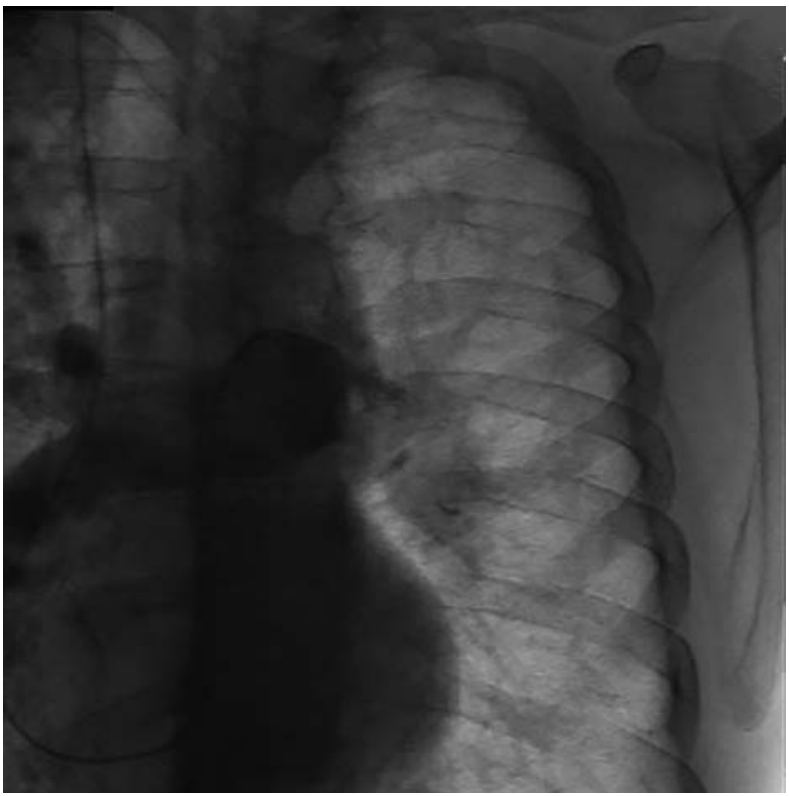

Figura 3. Angiografía pulmonar con oclusión de arteria pulmonar izquierda en origen y oclusión subsegmentaria derecha por completa.

bulo medio e inferior derechos, que permitieron interpretar el cuadro clínico como nuevo episodio de TEP derecho agudo, por lo que se decidió su internación

A su ingreso, se encontraba lúcido, hemodinámicamente estable, normotenso (presión arterial de 130/80 mmHg), frecuencia cardiaca de 85 latidos por minuto, taquipneico, con una saturación por oximetría de pulso de $88 \%$ al aire ambiente que corregía con oxígeno suplementario en bajas dosis. En la analítica del laboratorio presentó plaquetopenia (recuento de plaquetas: $91 \mathrm{mil} / \mathrm{mm}^{3}$ ) con el siguiente coagulograma: tiempo de protombina: 49\%, RIN (Rango internacional normatizado): 1,65; tiempo de tromboplastina parcial activado: 64 segundos. Se consensuó con el servicio de Hematología la anticoagulación con rivaroxaban dada la sospecha previa de HIT y ausencia de disponibilidad de fondaparinux y se procedió a la colocación de filtro de vena cava inferior. Se continuó su evaluación mediante angiografía pulmonar que reveló oclusión de arteria pulmonar izquierda y subsegmentaria de-

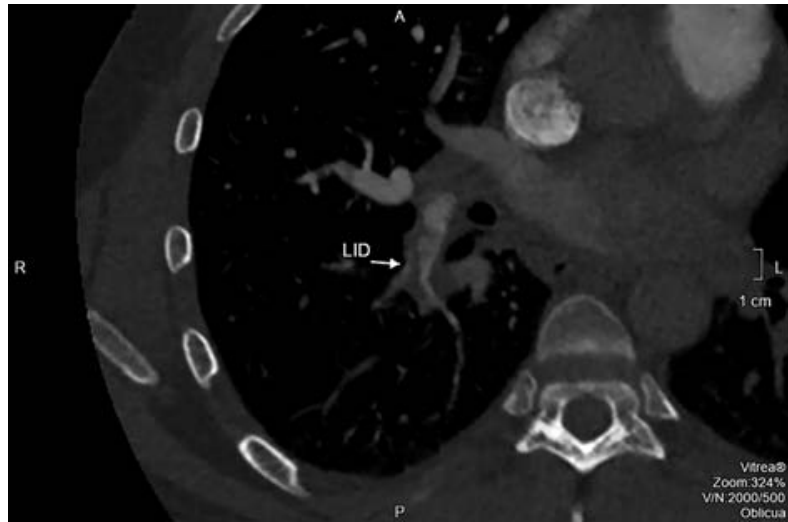

Figura 2. Tomografía computarizada con contraste endovenoso. Se evidencia defecto de relleno de contraste en la rama lobar inferior derecha compatible con tromboembolismo pulmonar agudo. LID: lóbulo inferior derecho

recha con HTP severa (Figura 3), cinecoronariografía con arterias coronarias sin lesiones angiográficamente significativas, con circulación colateral desde la arteria circunfleja hacia la circulación pulmonar. Se realizó un ecocardiograma Doppler que evidenció dilatación severa de las cavidades derechas con deterioro moderado de la función sistólica del ventrículo derecho, presión sistólica pulmonar de 88 mmHg e insuficiencia tricuspídea moderada. Los análisis de laboratorio confirmaron el diagnóstico de SAF con triple positividad de anticuerpos (anticoagulante lúpico, anticuerpos anti-ß2 Glicoproteína I y anti-cardiolipina positivos) y prueba de ELISA con antianticuerpos antifactor plaquetario 4 (anti-PF4) negativos. Luego de 8 días de internación, se otorgó el egreso hospitalario bajo tratamiento anticoagulante con rivaroxaban, con corticoides orales por interpretarse plaquetopenia de origen inmune y seguimiento ambulatorio por los servicios de Cardiología, Neumonología y Hematología.

A los 15 días del egreso, consultó nuevamente al departamento de emergencias por hemoptisis asociado a progresión de su disnea hasta CF IV Se realizó una nueva angiotomografía pulmonar que evidenció imagen compatible con infarto pulmonar hemorrágico (Figura 4). Se suspendió en dicho contexto la anticoagulación con rivaroxaban y luego de 3 días de estabilidad hemodinámica con recuento plaquetario en ascenso, se reinició anticoagulación con warfarina. Se reevaluó la angiografía previamente realizada en conjunto con el servicio de Hemodinamia y se procedió a la embolización con coils de ramas de circulación colateral de arteria mamaria izquierda hacia el hilio pulmonar izquierdo (Figura 5) Evolucionó favorablemente por lo que se decidió su externación.

Un mes más tarde fue internado en otro centro con diagnóstico de TEP agudo donde se indicó tratamiento anticoagulante con heparina sódica evolucionando con plaquetopenia, falleciendo 48 hs después de su ingreso por hemoptisis masiva.

\section{DISCUSIÓN}

La trombocitopenia inducida por heparina (HIT) es una de las complicaciones no hemorrágicas más importantes de la utilización de la heparina y sus derivados en la práctica clínica'. La misma se produce como consecuencia del desarrollo de anticuerpos contra complejos entre heparina y una proteína plaquetaria: el factor plaquetario 4. El desarrollo de estos an- 


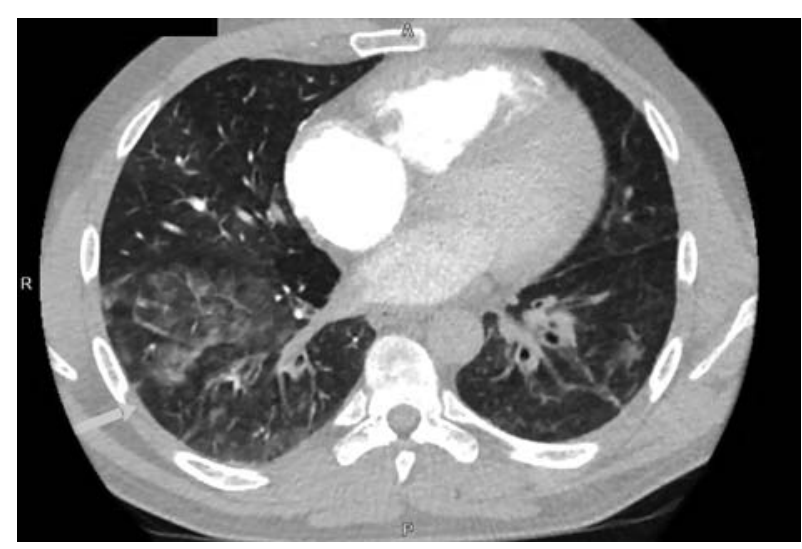

Figura 4. Signos de ocupación alveolar que se manifiestan como opacidades centroacinares en vidrio esmerilado en ambas bases pulmonares a predominio derecho que podrían estar en relación con hemorragia alveolar en primer término.

ticuerpos se produce en el 8 y $17 \%$ de los pacientes según la serie revisada ${ }^{1-4}$, alcanzando proporciones más elevadas en pacientes luego de cirugía cardíaca 5 . Sin embargo, la trombocitopenia y los fenómenos trombóticos venosos o arteriales son más infrecuentes, afectando del 0,1 al 5\% de los pacientes expuestos a la droga. Dichos anticuerpos son capaces de unirse al receptor plaquetario y leucocitario para el fragmento constante de las inmunoglobulinas lgG (FcyRlla), promoviendo la liberación de trombina, factor tisular y generando de esta forma una señalización intracelular que culmina con la activación plaquetaria y liberación de partículas procoagulantes. Uno de los scores más utilizados en la práctica clínica es el "4T's" con alta sensibilidad y alto valor predictivo negativo para aquellos pacientes categorizados con baja probabilidad de presentar la enfermedad ${ }^{6}$; actualmente otros scores se encuentran en revisión? 7 La sugerencia actual consiste en utilizar de forma conjunta dichos parámetros asociados a la demostración serológica de los anticuerpos mediante inmunoensayo o una prueba funcional con ensayos de activación plaquetaria. Para el tratamiento se deberá discontinuar el tratamiento con todo tipo de heparinas y evitar la re-exposición a las mismas, salvo en ciertos casos con seronegativización de los anticuerpos y necesidad de cirugía cardiovascular. Se indicará anticoagulación durante un período de tiempo variable según la condición clínica del paciente, siendo las opciones más evaluadas hasta la actualidad el argatrobán, bivalirudina, danaparoide y fondaparinux. Estos últimos no siempre se encuentran disponibles, surgiendo el interrogante de la utilidad de los anticoagulantes orales directos (DOACs) con experiencia en el tratamiento de múltiples patologías, dadas sus escasas interacciones farmacológicas, su administración en dosis fijas y la ausencia de necesidad de monitoreo rutinario. La evidencia actual para el HIT es escasa, surgiendo fundamentalmente de reportes de casos, siendo necesarios estudios prospectivos y multicéntricos que determinen su eficacia y seguridad ${ }^{8,9}$.

\section{BIBLIOGRAFÍA}

1. Arepally GM. Heparin-induced Thrombocytopenia. Blood 2017;129(21):28642872.

2. Warkentin TE, Levine MN, Hirsh J, Horsewood P, Roberts RS, Tech M, et Al. Hepa-

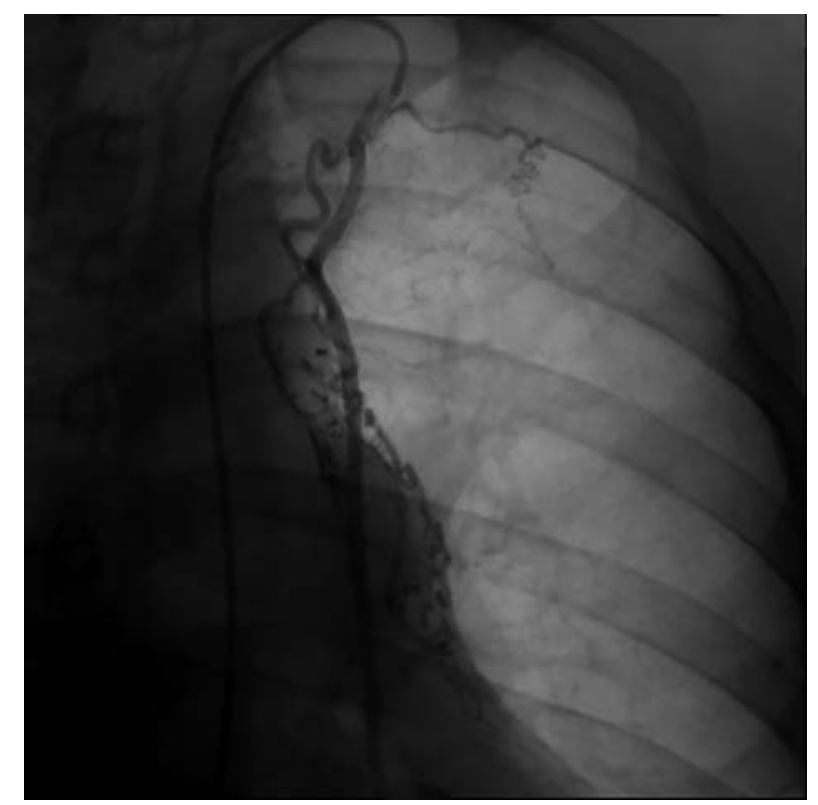

Figura 5. Angiografía de la arteria mamaria interna izquierda con circulación colateral hacia la arteria pulmonar izquierda.

EI SAF es una enfermedad sistémica adquirida que requiere para su diagnóstico la coexistencia de criterios clínicos, basados en fenómenos trombóticos, arteriales o de comorbilidades obstétricas, asociados criterios bioquímicos con positividad para uno o más anticuerpos antifosfolipídicos de forma sostenida en el tiempo ${ }^{10}$. Dada la elevada frecuencia de fenómenos trombóticos e incluso recurrencia de los mismos, el estándar de tratamiento consiste en su anticoagulación con AVK, siendo escasa la evidencia para la utilización de los DOACs ${ }^{11}$

La coexistencia de ambas enfermedades resulta extremadamente infrecuente $^{12,13}$, existiendo en la literatura sólo algunos reportes de casos.

En el paciente presentado, luego de la sospecha de HIT, se suspendió el tratamiento con heparinas, y dada la falta de disponibilidad de otras opciones terapéuticas como fondaparinux y argatrobán ${ }^{14}$, se debió iniciar anticoagulación con DOACs a pesar de la limitada evidencia en su utilización.

\section{CONCLUSIÓN}

La coexistencia del síndrome antifosfolipídico y trombocitopenia inducida por heparina es infrecuente. Este caso demuestra la ocurrencia de ambas entidades en un paciente. La evidencia relacionada con el abordaje y tratamiento de dichos pacientes es escasa y basada principalmente en reportes de casos y trabajos con series pequeñas de pacientes. Asimismo, refleja el difícil manejo de estos pacientes que obliga al abordaje multidisciplinario de la patología. 
duced Thrombocytopenia in a large, tertiary care teaching hospital. Chest 2007;131(6):1644-1649.

4. Salter BS, Weiner MM, Trinh MA, Heller J, Evans AS, Adams DH, et al. Heparin-Induced Thrombocytopenia: A Comprehensive Clinical Review. I Am Coll Cardiol 2016;67(21):2519-2532.

5. Trossaert M, Gaillard A, Commin PL, Amiral J, Vissac AM, Fressinaud E. High incidence of anti-heparin/platelet factor 4 antibodies after cardiopulmonary bypass surgery. Br J Haematol 1998;101(4):653-5.

6. Lo GK, Juhl D, Warkentin TE, Sigouin CS, Eichler P, Greinacher A. Evaluation of pretest clinical score (4 T's) for the diagnosis of heparin-induced thrombocytopenia in two clinical settings. J Thromb Haemost 2006;4(4):759-65.

7. Joseph L, Gomes MPV, Solaiman FA, St John J, Ozaki A, Raju M, et al. External Validation Of The HIT Expert Probability (HEP) Score, a Novel Pre-Test Probability Model For Heparin-Induced Thrombocytopenia Based On Broad Expert Opinion. Blood 2013;122(21):1699.

8. Seculini Patiño CE, Tabares AH. Podrían ser los anticoagulantes orales directos (ACODs) una alternativa para el tratamiento de la trombocitopenia inducida por heparina? Rev Fed Arg Cardiol 2016;45(1):8-12.
9. Warkentin TE, Pai M, Linkins LA. Direct oral anticoagulants for treatment of HIT: update of Hamilton experience and literature review. Blood 2017;130(9):11041113.

10. Chaturvedi S, McCrae KR. Diagnosis and management of the antiphospholipid syndrome. Blood Rev 2017;31(6):406-417.

11. Dufrost V, Risse J, Zuily S, Wahl D. Direct Oral Anticoagulants Use in Antiphospholipid Syndrome: Are These Drugs an Effective and Safe Alternative to Warfarin? A Systematic Review of the Literature. Curr Rheumatol Rep 2016;18(12):74.

12. Adediran S, Agostino N. Coexistence of Antiphospholipid Syndrome and Heparin-Induced Thrombocytopenia in a Patient with Recurrent Venous Thromboem bolism. Case Rep in Hematol 2017;2017:3423548.

13. Abd-Elsayed AA, Lisco SJ, Alsidawi S, Bonomo J. Simultaneous antiphospholipid syndrome and heparin-induced thrombocytopenia in a single patient. A A Case Rep 2014;2(1):9-10.

14. de Jesus GR, Agmon-Levin N, Andrade CA, Andreoli L, Chighizola CB, Porter TF, et Al. 14th International Congress on Antiphospholipid Antibodies Task Force report on obstetric antiphospholipid syndrome. Autoimmun Rev 2014;13(8):795-813. 


\title{
SÍNDROME DE TAKOTSUBO EN PACIENTE CON CARDIOPATÍA HIPERTRÓFICA
}

\author{
TAKOTSUBO SYNDROME IN PATIENT WITH HYPERTROPHIC \\ CARDIOMYOPATHY
}

FRANCO FACCIUTO' ${ }^{\prime}$ LEONARDO A. PILÓN' ${ }^{\prime}$, FRANCO L. MARINO', DANIELA F. CHILABERT ${ }^{2}$, FERNANDO BAGNERA ${ }^{3}$, GERARDO ZAPATA ${ }^{4}$

\begin{abstract}
RESUMEN
La cardiomiopatía de takotsubo (CT) es una condición aguda y reversible, caracterizada por una disfunción ventricular principalmente de los segmentos apicales del ventrículo izquierdo $(\mathrm{VI})$, con hipercontractilidad de los basales. Esto puede generar en ciertas ocasiones una obstrucción del tracto de salida del VI, lo que se puede exacerbar aún más en pacientes con cardiopatías hipertróficas. Debido a que la combinación de estas dos entidades es un escenario muy poco frecuente y a que su comportamiento en agudo muchas veces no es del todo benigno, su manejo puede ser extremadamente desafiante.

A continuación, se presenta el caso de un paciente de 72 años con hipertrofia ventricular izquierda y $\mathrm{CT}$ que evoluciona con shock cardiogénico.
\end{abstract}

Palabras clave: cardiomiopatía de takotsubo; cardiomiopatía hipertrófica; síndrome coronario agudo; shock cardiogénico.

\section{ABSTRACT}

Takotsubo cardiomyopathy (TCM) is an acute and reversible condition, characterized by ventricular dysfunction mainly of the apical segments of the left ventricle (LV), with hypercontractility of the basal cells. This can sometimes lead to obstruction of the LV outflow tract, which can be exacerbated even more in patients with hypertrophic heart disease. Because the combination of these two entities is a very rare scenario and because their behavior in the acute stage is often not entirely benign, their management can be extremely challenging.

Next, the case of a 72-year-old patient with left ventricular hypertrophy and TCM who progresses with cardiogenic shock is presented

Keywords: takotsubo cardiomyopathy; hypertrophic cardiomyopathy; acute coronary syndrome; cardiogenic shock.

REVISTA CONAREC 2018;33(146):255-258 | DOI:10.32407/RCON/2018146/0255-0258

\section{INTRODUCCIÓN}

La cardiomiopatía de takotsubo (CT), también conocida como síndrome de takotsubo, cardiomiopatía por estrés, síndrome de globo apical y síndrome del "corazón roto", es una condición clínica descrita por Sato en los años 90, caracterizada por una disfunción sistólica transitoria del ventrículo izquierdo (VI) que suele simular, al momento de presentarse, un infarto agudo de miocardio, con alteraciones electrocardiográficas en el segmento ST-T y discreto aumento de biomarcadores cardíacos ${ }^{1}$. Habitualmente es precedida por un desencadenante emocional o físico ocurriendo predominantemente en mujeres posmenopáusicas. Su patogenia no es conocida completamente aunque los mecanismos sugeridos en su génesis hacen referencia a mediación catecolaminérgica, espasmo coronario difuso, disfunción microvascular y miocardio aturdido neurogénico. El diagnóstico habitualmente es clínico, aunque en la mayoría de los casos se requiere una angiografía coronaria y una evaluación en serie de la fun-

1. Médico residente de Cardiología.

2. Jefe de Residentes de Cardiología

3. Médico Cardiólogo Staff de Unidad Coronaria.

4. Médico Cardiólogo Jefe de Unidad Coronaria. Instituto Cardiovascular de Rosario. Rosario, provincia de Santa Fe, Rep. Argentina.

$\triangle$ Correspondencia: Dr. Franco Facciuto. Bv Oroño 450, S2000DSR Rosario, Santa Fe, Rep.Argentina.facciutof@gmail.com

Los autores declaran no tener conflictos de intereses.

Recibido: 30/10/2018 | Aceptado: 10/11/2018 ción del $\mathrm{V}^{2,3}$. A pesar de que la recuperación completa de su función ocurre en 4 a 8 semanas, el riesgo de complicaciones intrahospitalarias graves como el shock cardiogénico (SC) es mayor en comparación con el infarto agudo de miocardio (11,4\% vs. $8 \%)$, evento que aumenta la mortalidad en 5 veces con respecto a aquellos sin SC 4,5 . Aunque este último puede ser secundario a disfunción sistólica, también puede ser causado por obstrucción del tracto de salida del VI (OTSVI), lo que se describió en el 10\% al 25\% de los pacientes con CT. Una posible hipótesis hace alusión a la marcada hiperdinamia de los segmentos basales, los cuales causarían un gradiente de presión intraventricular con un movimiento sistólico anterior de la valva mitral (MASVM), asociado a regurgitación mitral que conduciría a mayor colapso hemodinámico ${ }^{6}$.

La hipertrofia del VI es un fenómeno adaptativo muy frecuentemente diagnosticado en pacientes con hipertensión arterial de larga data y mal control. En dichas situaciones, adopta generalmente una distribución concéntrica y debe ser considerada una manifestación de daño de órgano blanco. En otras, puede estar relacionada con la presencia de trastornos genéticos (la mayoría autosómicos) conformando lo que se denomina miocardiopatía hipertrófica (MCH). Esta es la cardiopatía más frecuentemente relacionada a muerte súbita en los jóvenes y afecta en mayor medida al tabique ventricular (aunque la hipertrofia puede localizarse en cualquier región del VI). En dos tercios de los casos puede acompañarse de OTSVI "dinámica"7 y aún en pacientes sin gradiente en reposo puede generar una obstrucción "latente" desencadenada por factores adrenérgicos que alteran el estado hemodinámico. La ecocardiografía desempeña un papel importante en su diagnóstico: las imágenes bidimensionales son capaces 


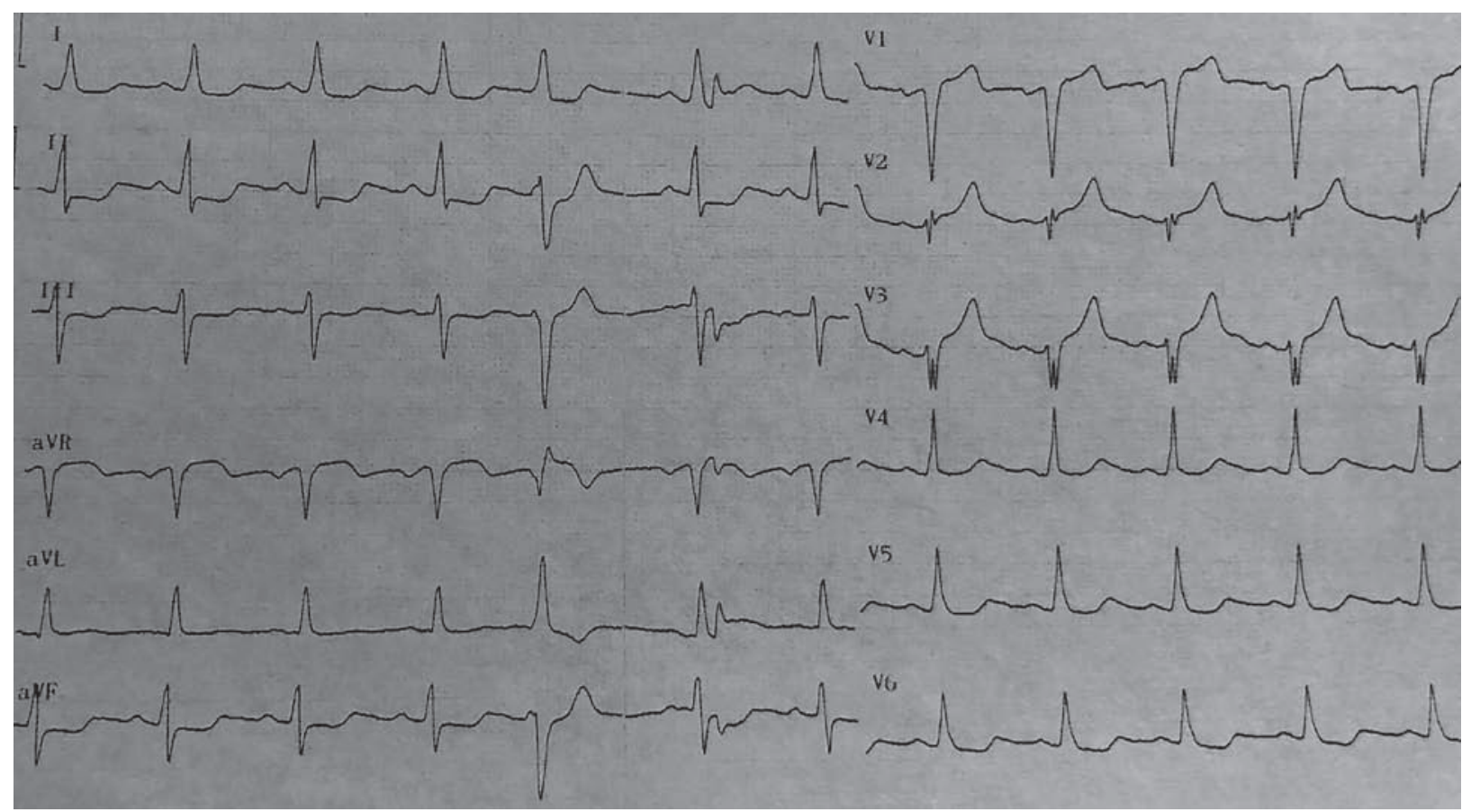

Figura 1. Electrocardiograma de ingreso: Se observa ritmo sinusal, con una frecuencia cardíaca de 75 latidos por minuto, supradesnivel del segmento ST de 6 milímetros de sumatoria de V1 a V3 e infradesnivel del segmento ST en DII, DIII y aVF.

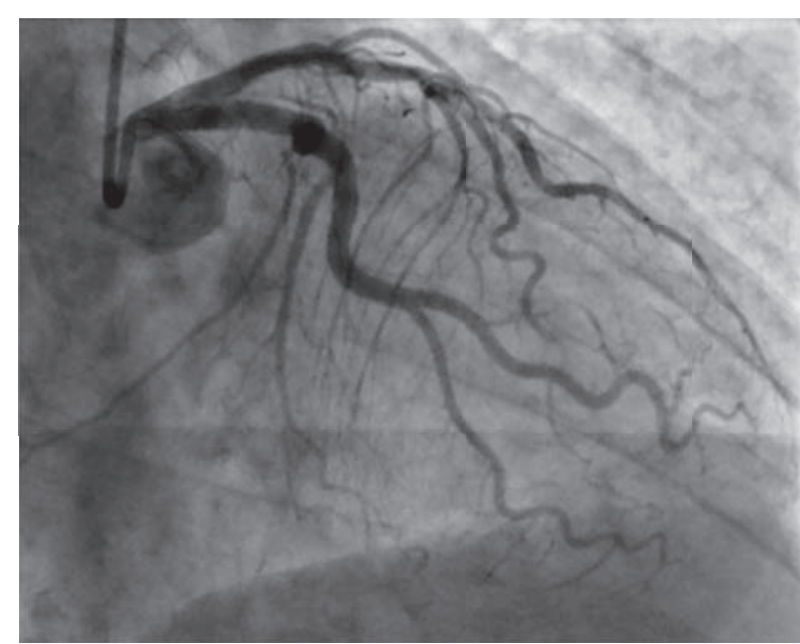

Figura 2. Cinecoronariografía que muestra ausencia de enfermedad coronaria epicárdica.

de cuantificar la magnitud de dicha hipertrofia, las vistas apicales de cuatro y cinco cámaras ayudan a identificar la gravedad y la duración del MASVM, y el Doppler color puede ayudar a localizar el nivel de obstrucción y a cuantificar su severidad ${ }^{8,9}$.

\section{CASO CLÍNICO}

Paciente de sexo masculino de 72 años, con hipertensión arterial en tratamiento con valsartán 160 mg y amlodipina 5 mg, sin antecedentes de enfermedad cardiovascular, que ingresó por dolor torácico de características opresivas de reposo, de 5 horas de evolución. Ante la sospecha etiológica de isquemia miocárdica aguda, se lo evaluó mediante electrocardiograma que evidenció supradesnivel del segmento ST de V1 a V3 con ondas Q en dicho terri- torio e infradesnivel inferolateral (Figura 1), por lo que se realizó cinecoronariografía de emergencia con el fin de adoptar eventual estrategia de reperfusión con angioplastia primaria pero la misma no mostró lesiones angiográficamente significativas (Figura 2), se realizó ventriculograma izquierdo que evidenció aquinesia apical con deterioro moderado a severo de la función sistólica del VI (Figura 3). Simultáneamente se solicitaron biomarcadores cardíacos, entre ellos troponina T de alta sensibilidad, que reflejaron valores marcadamente elevados (511,8 ug/l) por lo que se interpretó el cuadro como infarto agudo de miocardio con arterias coronarias epicárdicas angiográficamente normales y se inició tratamiento antiisquémico y antitrombótico. Se decidió continuar evaluación con ecocardiograma Doppler donde se encontró como datos positivos una marcada hipertrofia concéntrica del VI, con un septum y una pared posterior de 16 y $12 \mathrm{~mm}$, respectivamente, hipertrofia del septum basal (19 mm), una fracción de eyección del VI del 30\% con aquinesia apical en todos sus segmentos y del septum medio, y un gradiente medioventricular de reposo de 121 $\mathrm{mmHg}$ (Figura 4).

A las 8 horas de su estadía en unidad coronaria, evolucionó con hipotensión arterial sostenida y rales crepitantes en ambos campos pulmonares con radiografía de tórax con signos de redistribución de flujo, interpretándose el cuadro como SC. Se inició tratamiento con furosemida e inotrópicos (dobutamina y noradrenalina) durante 7 días, con buena respuesta clínica.

El paciente fue externado a los 13 días de su ingreso, con un electrocardiograma que mostraba negativización de ondas T en DI, aVL y de V3 a V6, previa evaluación con SPECT cardíaca de reposo para evaluar tamaño de infarto con perfusión normal en todos sus segmentos (tamaño de infarto de $0 \%$, fracción de eyección de $58 \%$ y volúmenes ventriculares normales) (Figura 5). 


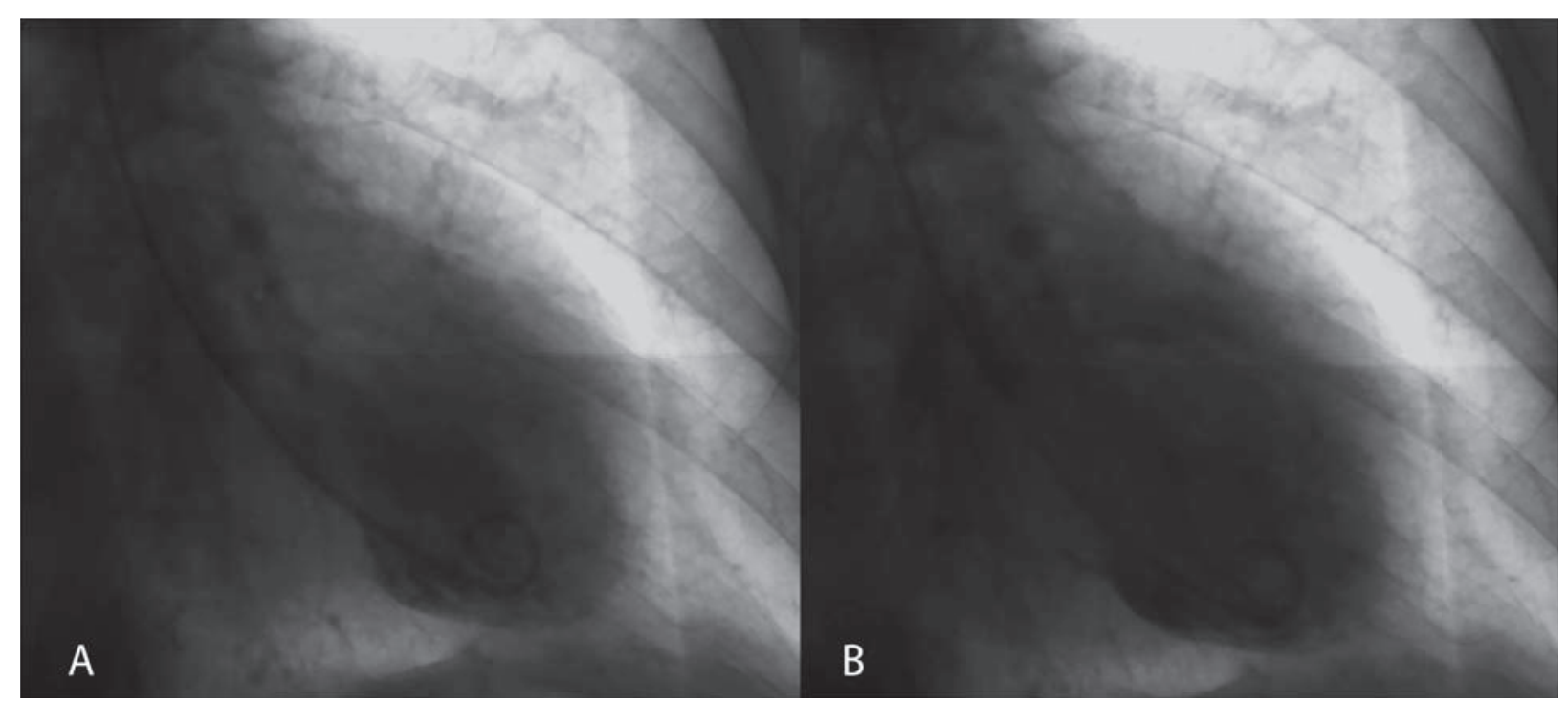

Figura 3. Ventriculograma izquierdo en sístole (A) y diástole (B) que muestra aquinesia apical con deterioro moderado a severo de la función sistólica del ventrículo izquierdo.

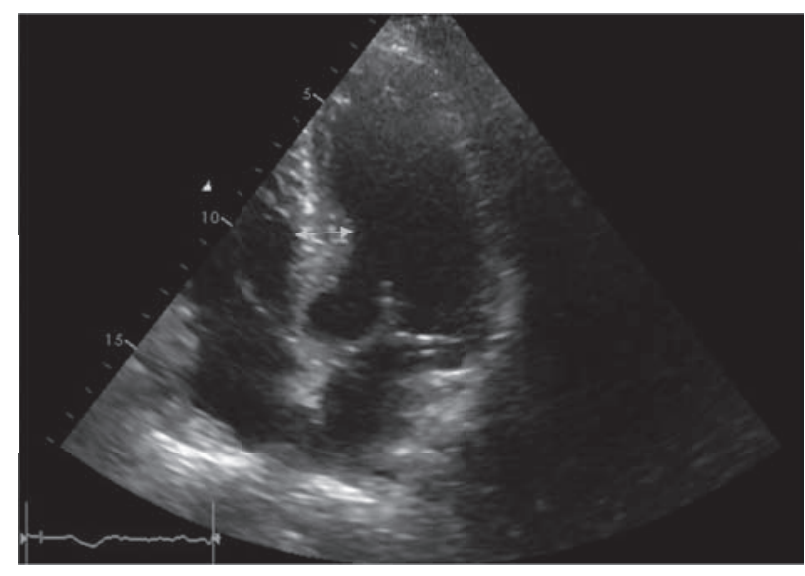

Figura 4. Ecocardiograma. Se muestra una vista apical de cuatro cámaras con hipertrofia septal (doble flecha).

\section{DISCUSIÓN}

Debido a su baja prevalencia, no es frecuente el reporte en la bibliografía de casos clínicos que combinen la presencia de CT en pacientes con MCH. En algunos la CT ocurrió en pacientes previamente diagnosticados con $\mathrm{MCH}^{10-13}$ y en otros la $\mathrm{MCH}$ no fue diagnosticada y la CT fue la causa de su enmascaramiento ${ }^{14-16}$.

Es clara la mayor prevalencia de CT en mujeres, aunque el sexo no descarta su diagnóstico, y en este caso no se identificó un factor estresante físico o emocional. Muchas veces los mismos pacientes minimizan estas cuestiones y tal vez no se pesquisó dicho dato en la anamnesis.

En el caso del paciente previamente presentado, tenía como antecedente a la hipertensión arterial, por lo que puede que se trate de una cardiopatía hipertensiva. Es llamativa su magnitud y disposición a nivel del septum basal y la presencia de gradiente medioventricular, datos raramente encontrados en cardiopatías hipertensivas, por lo que no podemos descartar que se trate de una MCH con hipertensión arterial asociada, aunque claro está que esta última serviría de factor

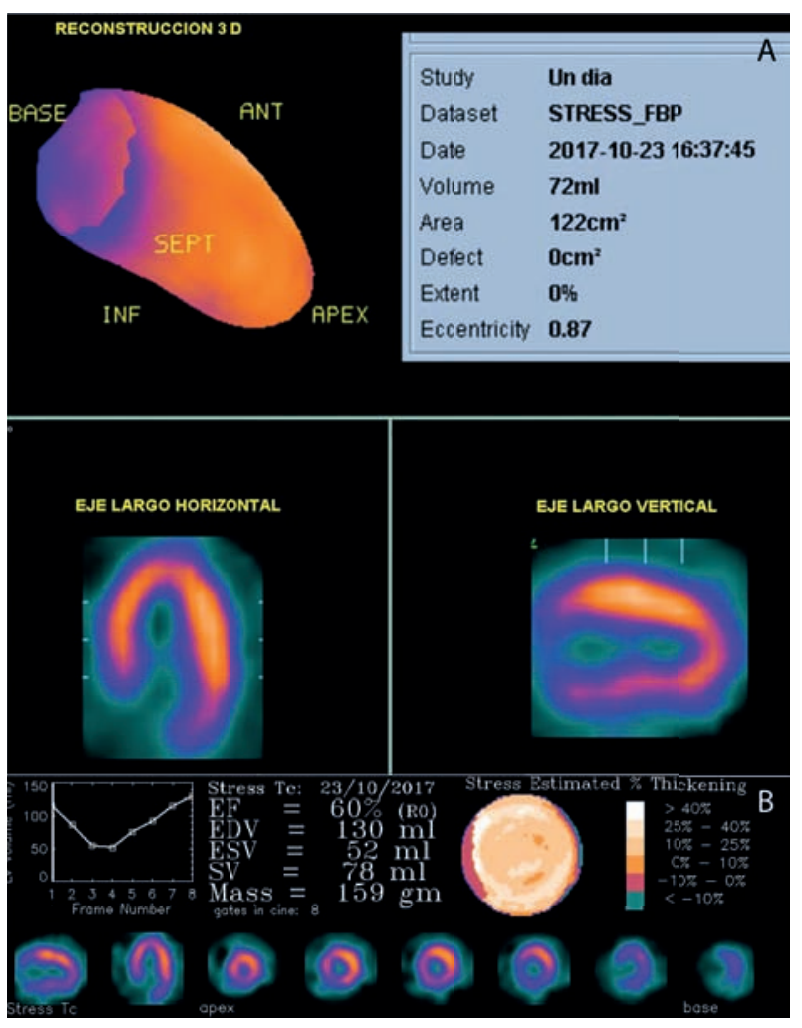

Figura 5. SPECT cardíaca de reposo. A. Normoperfusión en todos los segmentos. Tamaño infarto: $0 \%$. B. Fracción de eyección del ventrículo izquierdo conservada, con volúmenes ventriculares en límites normales.culares en límites normales.

para excluir a la primera ya que se la considera causa identificable de hipertrofia ventricular. Otra entidad descrita es el septum sigmoideo, también reconocido como hipertrofia septal localizada, nudillo o protuberancia septal. Sus causas y consecuencias son controvertidas; algunos lo consideran parte del espectro fenotípico de la $\mathrm{MCH}$, pero la mayoría, una entidad diferenciada y relacionada principalmente con una mayor edad, mayor angulación del septum interventricular respecto de la aorta o a hipertensión arterial. Clínicamente sue- 
le ser asintomático pero podría causar disnea de esfuerzo por OTSVI en algunas situaciones ${ }^{17}$. De igual manera, independientemente de la etiología, el manejo del paciente portador de esta condición no difiere y su complejidad sigue siendo sustancial.

La presencia de hipotensión en pacientes con CT debe evaluarse cuidadosamente con el fin de diferenciar bajo gasto secundario a disfunción ventricular izquierda del generado por OTSVI, ya que el uso de inotrópicos comunes puede empeorar el cuadro en este último caso. Aunque es un escenario muy poco frecuente, su tratamiento es extremadamente desafiante. La reanimación con líquidos (en ausencia de congestión pulmonar significativa) y los betabloqueantes son las opciones terapéuticas iniciales. Estas no fueron estrategias que pudimos aplicar ya que el paciente se encontraba claramente con signos de insuficiencia cardíaca retrógrada y anterógrada. Los agonistas alfa se pueden agregar para aumentar la presión arterial, lo que ayuda a disminuir el gradiente al aumentar la poscarga? .

\section{BIBLIOGRAFÍA}

1. Sato H, Tateishi H, Uchida T, et al. Takotsubo type cardiomyopathy due to multivessel spasm. In: Clinical aspect of myocardial injury: from ischemia to heart failure. Tokyo: Kagaku Hyoronsha 1990;56-64.

2. Ghadri J-R, Wittstein IS, Prasad A, Sharkey S, Dote K, Akashi YJ, et al. International Expert Consensus Document on Takotsubo Syndrome (Part 1): Clinical Characteristics, Diagnostic Criteria, and Pathophysiology. Eur Heart J 2018;39(22):2032-46.

3. Akashi YJ, Goldstein DS, Barbaro G, Ueyama T. Takotsubo cardiomyopathy: a new form of acute, reversible heart failure. Circulation 2008;118(25):2754-62.

4. Templin C, Ghadri JR, Diekmann J, Napp LC, Bataiosu DR, Jaguszewski M, et al. Clinical features and outcomes of Takotsubo (stress) cardiomyopathy. N Engl J Med 2015;373(10):929-938.

5. Almedro-Delia M, Núñez-Gil IJ, Lobo M, Andrés M, Vedia O, Sionis A, et al. Shortand Long-Term prognostic relevance of cardiogenic shock in Takotsubo Syndrome: Results from the RETAKO Registry. JACC Heart Fail 2018;6(11):928-36.

6. Fefer P, Chelvanathan A, Dick AJ, Teitelbaum EJ, Strauss BH, Cohen EA. Takotsubo cardiomyopathy and left ventricular outflow tract obstruction. J Interv Cardiol 2009;22(5):444-52

7. Elliott PM, Anastasakis A, Borger MA, Borggrefe M, Cecchi F, Charron P, et al. Guía de práctica clínica de la ESC 2014 sobre el diagnóstico y manejo de la miocardiopatía hipertrófica. Rev Esp Cardiol 2015;68(1):63.e1-e52.

8. Chockalingam A, Tejwani L, Aggarwal K, Dellsperger KC. Dynamic left ventricular outflow tract obstruction in acute myocardial infarction with shock: cause, effect, and coincidence. Circulation 2007;116(5):e110-3.

9. Akita K, Maekawa Y, Tsuruta H, Okuda S, Yanagisawa R, Kageyama T, et al. "Moving left ventricular obstruction" due to stress cardiomyopathy in a patient with hy-
Nuestro paciente complicó con SC que se trató con éxito con la administración gradual de distintos inotrópicos bajo un control hemodinámico cercano, y resolvió luego de nueve días sin requerir la colocación de dispositivos de asistencia ventricular.

Previo al alta se le solicitó una SPECT cardíaca de reposo que descartó áreas de fibrosis, avalando la fisiopatología planteada: un importante atontamiento miocárdico que resuelve a las pocas semanas.

\section{CONCLUSIÓN}

La combinación de cardiomiopatía de takotsubo y cardiopatía hipertrófica no es frecuentemente descripta por la bibliografía, por lo que conocer su expresión clínica resulta de gran interés en la práctica diaria teniendo en cuenta la complejidad de su manejo. pertrophic obstructive cardiomyopathy treated with percutaneous transluminal septal myocardial ablation. Int J Cardiol 2016;202:194-5.

10. Ochiumi Y, Ikeda S, Hamada M. Reappearance of the left ventricular pressure gradient in a patient with hypertrophic obstructive cardiomyopathy. Intern Med 2015;54(7):805-6.

11. Singh NK, Rehman A, Hansalia SJ. Transient apical ballooning in hypertrophic obstructive cardiomyopathy. Tex Heart Inst J 2008;35(4):483-4.

12. Jaber WA, Wright SR, Murphy J. A patient with hypertrophic obstructive cardiomyopathy presenting with left ventricular apical ballooning syndrome. J Invasive Cardiol 2006;18(10):510-2.

13. Patrianakos AP, Nyktari E, Parthenakis FI, Vardas PE. Reversible left ventricular apical ballooning after heavy alcohol consumption in a patient with hypertrophic cardiomyopathy. Int J Cardiol 2013;164(3):e29-31.

14. Roy RR, Hakim FA, Hurst RT, Simper D, Appleton CP. Two cases of apical ballooning syndrome masking apical hypertrophic cardiomyopathy. Tex Heart Inst $J$ 2014;41(2):179-83.

15. Daralammori Y, El Garhy M, Gayed MR, Farah A, Lauer B, Secknus MA. Hypertrophic obstructive cardiomyopathy masked by Tako-tsubo syndrome: a case re port. Case Rep Cardiol 2012;2012:486427.

16. Brabham WW, Lewis GF, Bonnema DD, Nielsen CD, O'Brien TX. Takotsubo cardiomyopathy in a patient with previously undiagnosed hypertrophic cardiomyopathy with obstruction. Cardiovasc Revasc Med 2011;12(1):70.e1-5.

17. Gentille-Lorente D, Salvadó-Usach T. Septo sigmoideo: ¿Una variante de la hipertrofia ventricular o de la miocardiopatía hipertrófica?. Arch Cardiol Mex 2016;86(2):110-122. 


\title{
CUANDO EL POSOPERATORIO SE COMPLICA
}

\author{
WHEN THE POSTOPERATIVE PERIOD GETS COMPLICATED
}

\section{IGNACIO A. VACA VALVERDE ${ }^{1}$, RAFAEL PORCILE², RICARDO LEVIN³ , JUAN FERNÁNDEZ ${ }^{4}$, FLAVIO SALVAGGIO ${ }^{5}$, ALEJANDRO BOTBOL ${ }^{6}$}

\section{RESUMEN}

El pseudoaneurisma aórtico (PSA) es una complicación reportada después de una cirugía cardíaca. La seguridad y eficacia de las terapias endovasculares para esta patología aún es controvertida. Sin embargo, el tratamiento quirúrgico de Ios PSA se asocia con una alta mortalidad y morbilidad, y en algunos casos no es factible, por lo que el cierre percutáneo se ha descrito como una alternativa.

Presentamos un caso de tratamiento endovascular de PSA después de reemplazo de válvula aórtica.

Palabras clave: cirugía torácica; aneurisma falso; aorta; reoperación; procedimientos endovasculares..

\section{ABSTRACT}

Aortic pseudoaneurysm (APA) is a commonly reported complication after cardiac surgery. The safety and efficacy of endovascular therapies for this pathology is still controversial. However, the surgical treatment of APA is associated with high mortality and morbidity, and in some cases it is not feasible, so percutaneous closure has been described as an alternative.

We present a case of endovascular APA treatment after aortic valve replacement.

Keywords: thoracic surgery, false aneurysm, aorta, reoperation, endovascular procedures.

REVISTA CONAREC 2018;33(146):259-260 | DOI:10.32407/RCON/2018146/0259-0260

\section{INTRODUCCIÓN}

El pseudoaneurisma aórtico (PSA) es una complicación reportada luego de cirugías torácicas, incluyendo cirugías de revascularización miocárdica, reemplazos valvulares, trasplantes cardiacos y reparaciones aórticas. Se asocia frecuentemente con la canulación del vaso, conllevando a un proceso curativo del endotelio no siempre efectivo y, como consecuencia, los defectos de la pared. Adicionalmente eventos traumáticos, inflamatorios e infecciosos pueden estar asociados a esta patología'. El gold standard de tratamiento de los PSA suele ser quirúrgico, pero en la mayoría de los casos puede resultar en una elevada morbimortalidad perioperatoria. Las terapias endovasculares surgen como alternativa terapéutica, aunque su eficacia y factibilidad no está suficientemente estudiada.

Presentamos el caso de un paciente con PSA posterior a reemplazo valvular aórtico tratado con endoprótesis aórtica.

\section{CASO CLÍNICO}

Paciente masculino de 67 años, con antecedentes de estenosis aórtica sintomática por disnea. Ingresó de forma programada para cirugía de reemplazo valvular aórtico con prótesis biológica. Presentó en

\footnotetext{
1. Residente de Cardiología Clínica.

2. Jefe del Servicio de Cardiología Clínica.

3. Coordinador de Unidad Cardiología Crítica.

4. Coordinador del Servicio de Hemodinamia y Cardiología Intervencionista.

5. Coordinador del Servicio de Imágenes cardiovasculares y ultrasonido. Hospital Universitario de la Universidad Abierta Interamericana.

$\triangle$ Correspondencia: Dr. Ignacio Vaca. Portela 2975, C1437BZK CABA, Rep. Argentina. nachovacavalverde@gmail.com.
}

Los autores declaran no tener conflictos de intereses.

Recibido: 10/11/2017 | Aceptado: 05/08/2018 el posoperatorio mediastinitis temprana, con rescate en cultivos de Staphyloccocus aureus meticilino resistente asociado a vegetación adyacente a prótesis valvular. Debido a la negativa del paciente a una nueva intervención quirúrgica se indicó tratamiento antibiótico, toilette mediastinal y cierre con colgajo pectoral. Evolucionó favorablemente, de forma asintomática, con ecocardiograma que evidenció disminución del tamaño de la vegetación.

El paciente reingresó a los 60 días por síndrome febril y shock hipovolémico secundario a sangrado profuso autolimitado de esternotomía requiriendo asistencia respiratoria mecánica y soporte transfusional. Se realizó una angiotomografía de tórax con sospecha de complicación vascular aórtica, con diagnóstico final de pseudoaneurisma de aorta ascendente. Se decidió en equipo multidisciplinario resolución endovascular con colocación de endoprótesis y stent autoexpandible (CP STENT, B. Braun Interventional Systems Inc. EE.UU.) por alto riesgo quirúrgico. Se realizó procedimiento de forma exitosa, sin presentar complicaciones, y fue dado de alta por buena evolución clínica. A los 90 días reingresó con nuevo episodio de shock hipovolémico, como consecuencia de un nuevo pseudoaneurisma anterior a la endoprótesis de aorta ascendente (Figura 1). Se realizó colocación de un dispositivo oclusor Amplatzer vascular Plug II, sin complicaciones inmediatas. Finalmente se otorgó el alta sanatorial con buena evolución en seguimiento.

\section{DISCUSIÓN}

Para los pacientes con riesgo elevado de reparación convencional de disección aórtica tipo A, pseudoaneurismas o disecciones crónicas, la reparación endovascular podría ofrecer una opción razonable al tratamiento médico². La morbilidad es significativamente alta, pero podría ser una opción de salvataje para pacientes seleccionados. Se podrían obtener mejores resultados con la adecuada selección de pacientes, y con el desarrollo de dispositivos específicos para esta patología ${ }^{3}$.

La proximidad de la válvula aórtica y las arterias coronarias a la aorta 


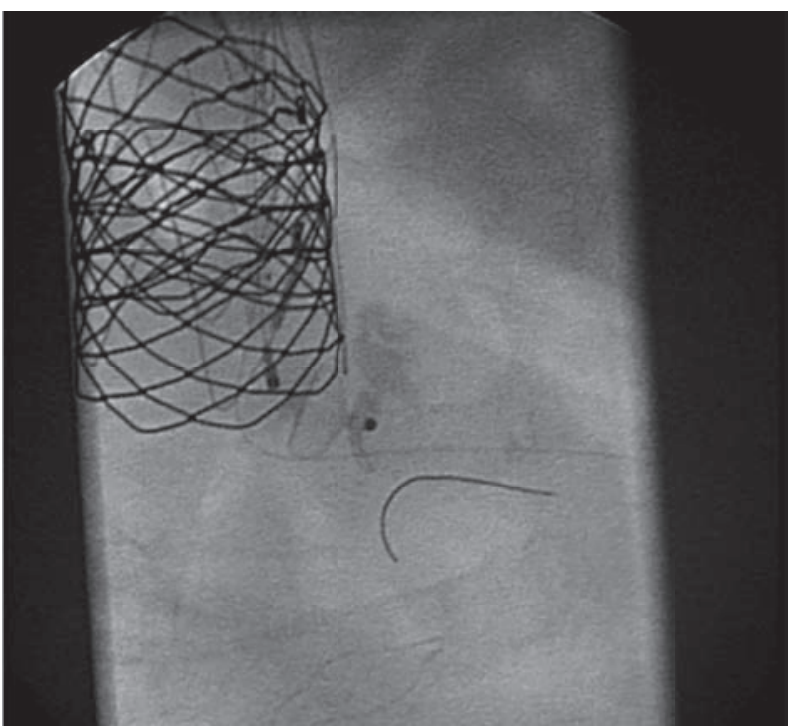

Figura 1. Angiotomografía de aorta. Flecha roja: pseudoaneurisma de aorta ascendente. Flecha celeste: stent graft colocado por primer pseudoaneurisma.

ascendente hace que la manipulación de esta área sea un verdadero desafío a la hora del sellado y la fijación de la prótesis a la aorta proximal. Es por este motivo que se diseñaron diversos dispositivos para su uso en el tratamiento de patologías aórticas ${ }^{4}$.

Existen disponibles dispositivos para stent grafting de aorta descenden.te, y algunos están aprobados para su uso en disecciones y aneurismas. La aorta ascendente difiere de la descendente en su tamaño, forma, fuerzas hemodinámicas y proximidad a ciertas estructuras anatómicas. Estas diferencias son importantes a considerar cuando se selecciona un paciente para manejo endovascular.

El cierre de PSA con el uso de coils ha sido reportado. Éstos se utilizan en la mayoría de los casos como terapia adjunta a stent grafts, dispositivos oclusores o plugs vasculares. La indicación para utilizar coils incluye que el PSA tenga un tamaño sacular pequeño y con cuello lo suficientemente reducido para evitar la regurgitación de coils a la circulación central. La forma de introducirlos es mediante un micro catéter especializado. Estos mismos se pueden utilizar para cualquier lugar anatómico ya que sus efectos mecánicos no difieren.

Una variedad de dispositivos han sido utilizados off-label para el cierre de PSA ${ }^{5}$, entre los cuales se encuentran los dispositivos oclusores septales, los dispositivos para cierre de foramen oval permeable, para defec-

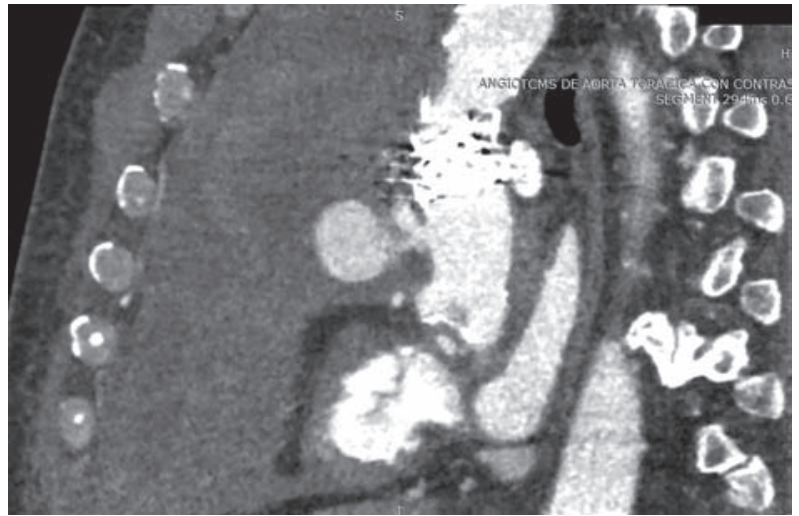

Figura 2. Colocación de dispositivo Amplatzer vascular Plug en pseudoaneurisma con guía angiográfica. Flecha roja: stent graft. Flecha Azul: Dispositivo plug vascular.

tos septales ventriculares, plugs de diferentes tipos e inclusive dispositivos para patologías congénitas. La elección del dispositivo debe ser individualizada según la recomendación de los expertos tomando siempre en cuenta la proximidad de los ostia coronarios al momento de la elección $n^{6}$. La práctica con estos dispositivos debe permanecer reservada para pacientes con antecedentes de múltiples intervenciones con requerimiento de toracotomía o riesgo quirúrgico alto. De los 40 casos reportados en la literatura, se ha descrito que en el 75\% se utilizaron dispositivos tipo plug con buenos resultados a corto plazo. En la serie de casos más grande reportada, se logró colocar el dispositivo sin complicaciones en el 80\% de los pacientes. En una serie del 2012 de 3 pacientes se reportó un seguimiento clínico a 6 meses sin eventos relacionados al implante ${ }^{7}$.

Los dispositivos disponibles en la actualidad no están adecuados a las características únicas que tiene la anatomía de la aorta ascendente. También por su curvatura existe una diferencia significativa entre las curvas mayores y menores. Las fuerzas hemodinámicas aumentadas en esta zona también representan una dificultad al momento de liberar los dispositivos y podrían comprometer su precisión de anclaje como también llevar a migraciones de la prótesis a largo plazo.

\section{CONCLUSIÓN}

El tratamiento quirúrgico de los PSA se asocia con una elevada morbimortalidad, constituyendo un desafío médico-quirúrgico. El manejo endovascular en este tipo de patologías surge como alternativa terapéutica, aunque su eficacia y factibilidad no está suficientemente estudiada.

\section{BIBLIOGRAFÍA}

1. Gray BH, Langan EM, Manos G, Bair L, Lysak SZ. Technical strategy for the endovascular management of ascending aortic pseudoaneurysm. Ann Vasc Surg 2012;26(5):734-738.

2. Kim SW, Lee DY, Kim MD, Won JY, Park SI, Yoon YN, et al. Outcomes of endovascular treatment for aortic pseudoaneurysm in Behcet's disease. I Vasc Surg 2014;59(3):608-614.

3. Vaughan-Huxley E, Hamady MS, Metcalfe MJ, Adams B, Kashef E, Cheshire NJ, et al. Endovascular repair of an acute, mycotic, ascending aortic pseudoaneurysm. Eur J Vasc Endovasc Surg 2011;41(4):488-491.

4. Saito N, Kimura T, Odashiro K, Toma M, Nobuyoshi M, Ueno K, et al. Feasibility of the Inoue single-branched stent-graft implantation for thoracic aortic aneurysm or dissection involving the left subclavian artery: short- to medium-term results in 17 patients. J Vasc Surg 2005:41(2):206-212.

5. Barbetakis N Xenikakis T, Efstathiou A, Fessatidis I. Percutaneous coil embolisation of a false aortic aneurysm following coronary surgery and mediastinitis. Hellenic J Cardiol 2007;48(4):246-248.

6. Faganello G, Hamilton M, Wilde P, Turner MS. Percutaneous closure of false aneurysms of the aorta in Wiskott Aldrich syndrome. Eur Heart J 2008;29(1):6.

7. Noble S, Ibrahim R. Embolization of an Amplatzer mVSD occluder device used for percutaneous closure of an ascending aortic pseudoaneurysm: case report and literature review. Catheter Cardiovasc Interv 2012;79(2):334-338.

8. Hussain J, Strumpf R, Wheatley G, Diethrich E. Percutaneous closure of aortic pseudoaneurysm by Amplatzer occluder device-case series of six patients. Catheter Cardiovasc Interv. 2009;73(4):521-529. 


\title{
REGLAMENTO DE PUBLICACIONES DE LA REVISTA DEL CONSEJO ARGENTINO DE RESIDENTES DE CARDIOLOGÍA
}

\author{
RULES OF PUBLICATIONS OF CONAREC JOURNAL
}

La Revista del Consejo Argentino de Residentes de Cardiología (CONAREC) publica artículos sobre temas relacionados con la Cardiología en su más amplio sentido. La Revista CONAREC es una publicación bimestral de marzo a diciembre (cinco números anuales) con objetivos asentados en la divulgación de material científico y educativo sobre la especialidad

La publicación es de tipo impresa y electrónica (www.revistaconarec.com.ar), ambas de acceso gratuito. La distribución se realiza a nivel nacional y está dirigida a residentes y concurrentes de Cardiología, así como a cardiólogos clínicos e intervencionistas, técnicos en Cardiología, centros asistenciales, asociaciones científicas, bibliotecas y facultades de Medicina.

Los principios editoriales de la revista se basan en las recomendaciones para manuscritos enviados a revistas Biomédicas (Recommendations for the Conduct, Reporting, Editing, and Publication of Scholarly Work in Medical Journals) redactadas por el Comité Internacional de Editores de Revistas Médicas (Internacional Comittee of Medical Journal Editors - ICMJE) en su más reciente actualización, disponible en www.icmje.org.

\section{ORIGINALIDAD DEL MATERIAL}

Los artículos enviados deben ser originales e inéditos. No serán aceptados trabajos que se encuentren publicados o en evaluación en otras revistas científicas tanto en el mismo como en distinto idioma.

Artículos duplicados. Se consideran artículos duplicados aquellos que contienen material que ya ha sido publicado en su totalidad o en gran parte, o se encuentra incluido en o estrechamente vinculado a otro trabajo que ha sido enviado o aceptado para su publicación en otra revista. Estos artículos no serán aceptados para su publicación

Publicaciones secundarias. La publicación secundaria de material publicado en otras revistas puede ser justificable y beneficiosa, especialmente cuando se intenta transmitis información relevante a la mayor audiencia posible (ej: guías de práctica clínica, registros con datos nacionales sobre patologías prevalentes en materia de salud pública). Los artículos se considerarán para publicación secundaria siempre y cuando se ajusten a las recomendaciones ICMJE y los editores de ambas revistas lo aprueben Además debe ser debidamente aclarado en el texto que ya ha sido publicado en todo o en parte y deber citarse adecuadamente la publicación original.

\section{ÉTICA}

Los trabajos clínicos experimentales que sean enviados para su evaluación deben elaborarse respetando las recomendaciones internacionales sobre investigación clínica desarrolladas en la Declaración de Helsinki de la Asociación Médica Mundial revisada recientemente (www.wma.net/es/policy) y deben haber sido aprobados por un Comité de Ética institucional o regional responsable en experimentación humana. En el caso de utilización de animales de laboratorio, deberá ajustarse a las normas de la Sociedad Americana de Fisiología. Los autores de los artículos aceptan la responsabilidad definida por el Comité Internacional de Editores de Revistas Médicas (www.icmje.org).

\section{PROTECCIÓN DE LA PRIVACIDAD DE LOS PACIENTES}

No pueden publicarse descripciones, fotografías u otros detalles que contribuyan a identificar al paciente, a menos que esta información sea indispensable para la exposición del material; en ese caso, el paciente o el padre o tutor de los menores de edad expresarán su consentimiento por escrito, el cual deberá adjuntarse.

\section{REGISTRO DE ENSAYOS CLÍNICOS}

La Revista CONAREC apoya las políticas para registro de ensayos clínicos de la Organización Mundial de Salud (OMS) y del ICMJE, reconociendo la importancia de esas iniciativas para el registro y divulgación internacional de información sobre estudios clínicos, en acceso abierto. En consecuencia, solamente se aceptarán para publicación los artículos de investigaciones clínicas que hayan recibido un número de identificación en uno de los Registros de Ensayos Clínicos validados por los criterios establecidos por la OMS e ICMJE. El número de identificación se deberá consignar al final del resumen.

\section{SECCIONES}

\section{Artículos originales}

Son trabajos científicos o educativos de investigación básica o clínica original. Debe seguir la estructura "IMRD", es decir, debe tener Introducción, Material y métodos, Resultados y Discusión (véanse las normas de publicación ICMJE). Además son necesarias una Conclusión y Referencias bibliográficas. Cuando la situación lo amerite, se pueden agregar Agradecimientos y un Apéndice adjunto.

Condiciones: texto general hasta 5.000 palabras, resumen hasta 500 palabras, 3-6 palabras clave, tablas más figuras hasta 8, referencias hasta 40, autores hasta 10.

\section{Revisión anual}

La Revisión Anual consiste en un tema relevante de la especialidad que será desarrollado durante todo el año en cada volumen. Será escrita por profesionales destacados, Miembros Honorarios o del Comité Asesor del CONAREC a pedido del Comité de Redacción. Condiciones: texto general hasta 5.000 palabras, resumen hasta 500 palabras, 3-6 palabras clave, tablas más figuras hasta 8, referencias hasta 40, autores hasta 3.

\section{Revisión por expertos}

Son artículos sobre temas relevantes de la especialidad solicitados por el Comité de Redacción a autores de reconocida trayectoria (nacionales o extranjeros). Excepcionalmente podrán ser considerados para publicación artículos no solicitados por el Comité siempre y cuando se ajusten al presente reglamento.

Condiciones: texto general hasta 5.000 palabras, resumen hasta 500 palabras, 3-6 palabras clave, tablas más figuras hasta 8, referencias hasta 40, autores hasta 3.

\section{Médicos de Guardia}

Son artículos sobre el manejo racional y protocolizado de diferentes circunstancias que se presentan en la práctica diaria. Son revisados y consensuados previamente con especialistas en el tema, que culminan con un diagrama de flujo sobre el manejo diagnóstico-terapéutico de la patología.

Condiciones: texto general hasta 3.000 palabras, resumen hasta 150 palabras, tablas más figuras hasta 6, referencias hasta 20, autores hasta 8

\section{Caso clínico}

Es la descripción de un caso clínico de características inusuales, que incluye su abordaje diagnóstico y terapéutico, y su resolución final. Debe acompañarse de una introducción, una discusión bibliográfica y las conclusiones pertinentes.

Condiciones: texto general hasta 2.000 palabras, resumen hasta 350 palabras, tablas más figuras hasta 5, referencias hasta 20, autores hasta 10

\section{Imágenes en Cardiología}

Son imágenes, no necesariamente excepcionales, pero sí ilustrativas, que deben ir acompañadas de una leyenda explicativa, un resumen del caso clínico y una breve reseña bibliográfica. Condiciones: texto general hasta 1.200 palabras, resumen hasta 350 palabras, figuras hasta 8 , referencias hasta 10 , autores hasta 10.

\section{Editoriales}

Son comentarios y/o análisis de un artículo publicado en el número de la revista en el que aparece y es solicitado por el Comité de Redacción a un autor experto en el tema. Asimismo, pueden solicitarse comentarios sobre temas no relacionados con un artículo en particular.

Condiciones: texto general hasta 1.200 palabras, referencias hasta 10.

\section{Monografía seleccionada}

Son monografías seleccionadas y/o premiadas por el Comité Científico de la última Jornada Interresidentes de Cardiología, adaptadas para la publicación en la revista (ver normas de publicación ICMJE)

Condiciones: texto general hasta 10.000 palabras, resumen hasta 500 palabras, 3-6 palabras clave, tablas más figuras hasta 10 y referencias hasta 100.

\section{Selección de artículos relevantes, guías y consensos publicados}

La selección del material estará a cargo de miembros del Comité de Redacción teniendo en cuenta las principales revistas nacionales e internacionales.

Condiciones: se confeccionará una lista en la que conste el título del artículo seleccionado y la correspondiente cita de la revista en la que fue publicado según las normas generales de publicación de CONAREC

\section{Agenda CONAREC}

Se publicarán las actividades más importantes correspondientes al bimestre de la edición. 


\section{RECEPCIÓN, EVALUACIÓN Y PUBLICACIÓN DE ARTÍCULOS}

El envío del artículo (texto, tablas y figuras) debe realizarse por correo electrónico a conarecrevista@gmail.com consignando en el asunto la sección a la que corresponda.

Cada manuscrito recibido será examinado por el Comité de Redacción con la su pervisión del Comité Asesor y de ser adecuado a las normas de publicación será evaluado por dos árbitros externos especializados en el tema en forma doble ciego: el material será enviado a estos últimos sin consignar el nombre de los autores ni el centro al que pertenecen. Si los revisores consideran necesaria la realización de modificaciones, se enviarán las sugerencias al autor responsable preservando la identidad del revisor. El autor recibirá una respuesta preliminar dentro de los 3 meses de remitido el manuscrito correctamente, debiendo realizar los cambios sugeridos a la brevedad y reenviar el material para su nueva evaluación. Finalmente, se notificará al autor responsable sobre la aceptación o el rechazo del manuscrito.

El Comité de Redacción se reserva el derecho de introducir, con el consentimiento de los autores, todos los cambios editoriales exigidos por las normas gramaticales y las necesidades de edición de la revista.

Aprobada la publicación del trabajo, CONAREC retiene los derechos de autor para su reproducción total o parcial.

\section{PREPARACIÓN DEL MANUSCRITO}

El artículo debe estar escrito en castellano, en un procesador de texto Word (Microsoft ${ }^{\oplus}$ ) o similar (con formato compatible) y guardado con extensión * doc 0 *.docx. El tamaño de la página debe ser A4 o carta, con doble espacio interlineado, márgenes de 25 mm con texto justificado y con tamaño de letra de 12 puntos tipo Times New Roman. Las páginas se numerarán en forma consecutiva comenzando con la portada.

Cada artículo debe ser presentado con una primera página que debe contener: a) título del artículo en mayúscula con negrita; b) apellido y nombres completos de los autores; c) institución en que se desempeñan; d) cargos que ocupan; e) título abreviado para cabeza de página; f) título en inglés; g) número total de palabras del artículo, sin las referencias bibliográficas; h) número de palabras del resumen; i) nombre y dirección completa, código postal y dirección de correo electrónico del autor con quien se deba mantener correspondencia; j) declaración de la existencia o no de conflictos de intereses.

Para consignar los nombres de los autores, se debe colocar el apellido seguido por el nombre de pila y la inicial del segundo si lo tuviere, separado por punto y coma de siguiente (por ejemplo: Herrera Paz Juan J; Thierer Jorge). Continuado con punto seguido el lugar donde se realizó el trabajo. Debajo se debe colocar el lugar donde desempeña su tarea laboral y cargo que ocupa cada uno de los autores señalado con notas al pie, usando números consecutivos. Quienes figuren como autores deben habe participado en la investigación o en la elaboración del manuscrito y hacerse públicamente responsables de su contenido, adaptándose a las normas para la autoría expuestas por la IMCJE.

La segunda página debe incluir a) resumen en español; b) palabras clave en español; c) resumen en inglés americano (abstract); d) palabras clave en inglés (keywords); e) número de identificación en el registro de Ensayos Clínicos cuando corresponda. Las palabras clave deben ser términos incluidos en la lista del Index Medicus (Medical Subject Headings - MeSH) y Descriptores en Ciencias de la Salud (DeCS). Para la selección de estos se recomienda visitar los siguientes enlaces: http://www.nlm.nih.gov/ mesh/2014/mesh_browser/MBrowser y http://decs.bvs.br

Luego, en la tercera página, se debe desarrollar el contenido del manuscrito de acuerdo con las especificaciones de cada tipo de artículo, iniciando una nueva página para cada sección. Cada sección de la estructura "IMDR" debe ir con negrita mayúscula, mientras que las siguientes subsecciones dentro de la estructura IMDR deben ir con negrita tipo título separadas de las secciones por espacio simple.

\section{Unidades de medida}

Como unidades de medida se utilizarán las del sistema métrico decimal, usando comas para los decimales. Todas las mediciones clínicas, hematológicas y químicas deben ex presarse en unidades del sistema métrico y/o Ul, aclarando, cuando sea necesario, los rangos de referencia del laboratorio que realizó las determinaciones.

\section{Abreviaturas}

Solo se utilizarán las abreviaturas comunes, evitándose su uso en el título y en el resumen. La primera vez que se empleen irán precedidas por el término completo, excepto que se trate de unidades de medida estándar.

\section{Tablas y Figuras}

Las tablas y figuras deben presentarse en hojas individuales y se enumerarán consecutivamente con números arábigos (1, 2, etc.) según el orden que fueron citadas en el texto, con un título breve para cada una de ellas. Deben ser rotuladas con la palabra Tabla o Figura en negrita continuada por el número correspondiente de figura o tabla. Todas las abreviaturas de la tabla no estandarizadas deben explicarse. Las notas aclaratorias deben ir al pie de la tabla utilizando los siguientes símbolos en esta secuencia: * $\dagger, \neq, \S, \eta^{* * *}, \dagger+, \neq \neq$, etc

Las figuras deben estar en formato TIFF, PSD o JPEG a 300 dpi en formato final. Deben ser numeradas correlativamente con una leyenda explicativa en hoja aparte. El tamaño usual de las fotografías debe ser de 127×173 mm. Los títulos y las explicaciones detalladas se colocan en el texto de las leyendas y no en la ilustración misma.

\section{Referencias bibliográficas}

Las referencias bibliográficas solo se limitarán a citar a aquellos artículos originales directamente relacionados con el trabajo, evitándose mencionar artículos de revisión sobre el tema expuesto. Se enumerarán las referencias de manera consecutiva con números arábigos entre paréntesis. Se incluirán todos los autores cuando sean seis o menos; si fueran más de seis, el sexto será seguido de la expresión "et al". Los títulos de las revistas serán abreviados según el estilo empleado en el Index Medicus. Ejemplos según tipo de publicación a citar:

1. Registro de Procedimientos Diagnósticos y Terapéuticos efectuados durante el período 2006-2007. Colegio Argentino de Cardioangiólogos Intervencionistas (CACI). Disponible en http://www.caci.org.ar/addons/3/158.pdf. Consultado e 01/01/2009. (Para páginas web)

2. Ambrosy AP, Vaduganathan M, Huffman MD, Khan S, Kwasny MJ, Fought AJ, et al. Clinical course and predictive value of liver function tests in patients hospitalized for worsening heart failure with reduced ejection fraction: an analysis of the EVEREST trial. Eur J Heart Fail. 2012;14(3):302-11. (Para revistas en inglés).

3. Guardiani F, Mana M, Vázquez R. Trombosis simultánea en el infarto agudo de miocardio. Pancoronaritis. Rev Conarec. 2008;30(96):290-92. (Para revistas en español).

4. Braverman A, Thompson R, Sanchez L. Enfermedades de la aorta. En: Bonow R, Mann D, Zipes D, Libby P. Braunwald, Tratado de Cardiología: texto de medicina cardiovascular (2013, Novena edición, 1324-1354). España: Editorial Elsevier. (Libro).

\section{APÉNDICE - ESTRUCTURA"IMRD"}

Introducción. Establece el propósito del artículo y realiza el resumen de los fundamentos lógicos para la observación del estudio. Da únicamente las referencias estrictamente pertinentes. Se presentan los objetivos del trabajo, y se resumen las bases para el estudio o la observación. No debe incluir resultados o conclusiones del trabajo.

Materiales y métodos. Incluye una descripción de: a) La selección de los sujetos estudiados y sus características. b) Los métodos, aparatos y procedimientos; en estudios clínicos se informarán detalles del protocolo (población estudiada, intervenciones efectuadas, bases estadísticas). c) Guías o normas éticas seguidas. d) Descripción de los métodos estadísticos utilizados. Describe claramente la selección de los sujetos destinados a la observación y la experimentación (pacientes o animales de laboratorio, incluido grupo de control). Debe identificar edad, sexo y otras características relevantes de la población, los métodos, aparatos (proporcionar el nombre, dirección de la empresa que lo produce) y procedimientos con suficientes detalles que permitan a otros investigadores la reproducción de los resultados. Deben mencionarse las drogas y las sustancias químicas, incluidos nombre químico, dosis y vías de administración.

Dentro de esta sección deberá aclararse, si correspondiera, el análisis estadístico realizado, así como el programa utilizado para ello, y el nivel de significancia preestablecido. Los trabajos clínicos aleatorizados (randomizados) deberán presentar información sobre los elementos más importantes del estudio, que contengan el protocolo y la hoja de flujo de la inclusión de los pacientes, y además deberán seguir los lineamientos de CONSORT (consúltese el artículo en la página web de instrucciones de la revista).

Los autores que presentan revisiones deberán incluir una sección en la que se describan los métodos utilizados para la ubicación, la selección y la síntesis de datos; estos métodos deberán figurar abreviados en el resumen.

Resultados. Los resultados deben presentarse con una secuencia lógica en el texto, las tablas y las ilustraciones. No se deben repetir en el texto todos los datos de las tablas 0 las ilustraciones, debiendo destacar solo las observaciones importantes. Las tablas y las figuras deben utilizarse en el número estrictamente necesario para explicar el materia y para valorar su respaldo. Se recomienda emplear gráficos como alternativa para las tablas con numerosas entradas.

Discusión. Resalta los aspectos nuevos e importantes del estudio, las conclusiones de ellos derivadas, y su relación con los objetivos que figuran en la Introducción. No se debe repetir información que ya figure en otras secciones del trabajo. Evitar declaraciones de prioridad y referencias a trabajos aún no completados. Incluir los hallazgos, sus implicaciones y limitaciones, incluso lo que implicaría una futura investigación. Relacionar las observaciones con las de otros estudios importantes.

Las conclusiones deben estar relacionadas con los objetivos del estudio. Se deben evitar informes no calificados y conclusiones que no estén completamente respaldados por los datos. Los autores deben evitar dar informaciones sobre costos-beneficios económicos a menos que el artículo incluya datos económicos y su análisis. Plantear otras hipótesis cuando esté justificado, pero rotuladas claramente como tales. Las recomendaciones pueden incluirse cuando resulten apropiadas.

Agradecimientos. Precediendo a la bibliografía; si cabe se citarán: reconocimiento por apoyo técnico, aportes financieros, contribuciones que no lleguen a justificar autoría. En estos casos los autores serán responsables de contar con el consentimiento escrito de las personas nombradas.

Apéndice. En esta sección, de carácter opcional, deberá incluirse todo aquel material no contemplado en los apartados previos, y que resulte necesario o facilite la comprensión del manuscrito remitido. Ejemplo de esto son los formularios empleados para llevar adelante una encuesta, ilustraciones de una maquinaria empleada para una determinada tarea, o similar. 